\title{
Control of the Chemoselectivity of Metal $N$-Aryl Nitrene Reactivity: C-H Bond Amination versus Electrocyclization
}

\author{
Chen Kong, ${ }^{1, a}$ Navendu Jana,${ }^{1, a}$ Crystalann Jones, ${ }^{1}$ Tom G. Driver ${ }^{1,2 *}$ \\ ${ }^{1}$ Department of Chemistry, University of Illinois at Chicago, 845 West Taylor Street, Chicago, Illinois, USA, \\ 60607-7061 \\ ${ }^{2}$ Institute of Next Generation Matter Transformation, College of Chemical Engineering, Huaqiao University, 668 Jimei \\ Blvd. Xiamen, Fujian, 361021, P. R. China \\ ${ }^{\text {a }}$ These individuals contributed equally to this manuscript
}

\section{Contents:}

\section{Supporting Information}

I. Synthesis of 2-Azidoarylboronic Acid Pinacolate Esters..................................................................2

II. Synthesis of Trisubstituted Styryl Anilines....................................................................................

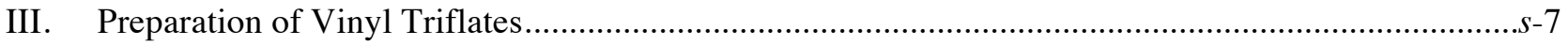

IV. Synthesis of 2-Alkenylaryl Amines by Suzuki Cross-Coupling Reaction.........................................s-18

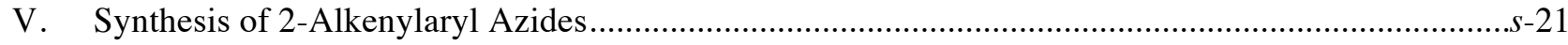

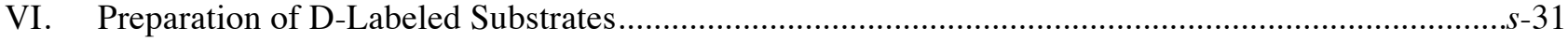

VII. Investigation of Catalyst Control of Site Selectivity ….................................................................s-34

VIII. $\quad \mathrm{Rh}_{2}(\mathrm{II})-\mathrm{Catalyzed} \mathrm{sp}^{3} \mathrm{C}-\mathrm{H}$ Bond Amination and Electrocyclization..............................................s-35

IX. Mechanistic Study for $\mathrm{Rh}_{2}(\mathrm{II})$-Catalyzed Electrocyclization and $\mathrm{sp}^{3} \mathrm{C}-\mathrm{H}$ Bond Amination..............s-40

X. Mechanistic Study for Fe(OEP)Cl-Catalyzed $\mathrm{sp}^{3} \mathrm{C}-\mathrm{H}$ Bond Amination.........................................s-51

XI. $\quad \mathrm{Fe}(\mathrm{OEP}) \mathrm{Cl}$-Catalyzed Synthesis of $2 \mathrm{H}$-Indoles.......................................................................5

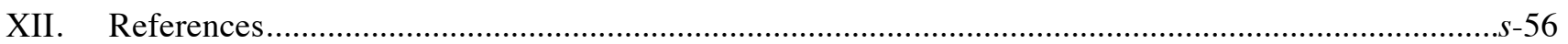

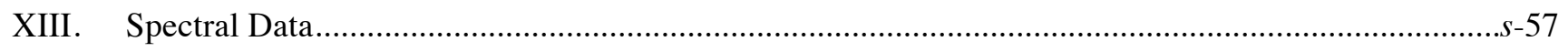

General. ${ }^{1} \mathrm{H}$ NMR and ${ }^{13} \mathrm{C}$ NMR spectra were recorded at ambient temperature using $500 \mathrm{MHz}$ or $300 \mathrm{MHz}$ spectrometers. The data are reported as follows: chemical shift in $\mathrm{ppm}$ from internal tetramethylsilane on the $\mathrm{d}$ scale, multiplicity $(\mathrm{br}=$ broad, $\mathrm{s}=$ singlet, $\mathrm{d}=$ doublet, $\mathrm{t}=$ triplet, $\mathrm{q}=$ quartet, $\mathrm{m}=$ multiplet $)$, coupling constants $(\mathrm{Hz})$ and integration. High-resolution mass spectra were obtained by peak matching. Melting points are reported uncorrected. Analytical thin layer chromatography was performed on $0.25 \mathrm{~mm}$ silica gel plates with UV254 fluorescent indicator. Liquid chromatography was performed using forced flow (flash chromatography) of the indicated solvent system on $60 \AA$ $(40-60 \mu \mathrm{m})$ mesh silica gel $\left(\mathrm{SiO}_{2}\right)$. Medium pressure liquid chromatography (MPLC) was performed using pumps to force flow the indicated solvent system down columns that had been packed with $60 \AA(40-60 \mu \mathrm{m})$ mesh silica gel $\left(\mathrm{SiO}_{2}\right)$. All reactions were carried out under an atmosphere of nitrogen in glassware that was oven-dried. Unless otherwise noted, all reagents were commercially obtained and, where appropriate, purified prior to use. Acetonitrile, methanol, toluene, THF, $\mathrm{Et}_{2} \mathrm{O}$, and $\mathrm{CH}_{2} \mathrm{Cl}_{2}$ were dried by filtration through alumina according to the procedure of Grubbs. ${ }^{1}$ Metal salts were stored in a nitrogen atmosphere dry box. CAUTION should be exercised handling organic azides because they are potentially explosive substances. 


\section{Synthesis of 2-Azidoarylboronic Acid Pinacolate Esters.}

\section{A. Substrate Synthesis Overview.}

The 2-azidoarylboronic acid pinacolate ester reagents were constructed from substituted 2-bromoanilines following the process outlined in Scheme s1. Palladium-catalyzed borylation of substituted 2-bromoanilines was performed following the conditions reported earlier by us. ${ }^{2}$ Azidation of s1 using trimethylsilyl azide following the conditions reported by Zhang and Moses produced the requisite 2-azidoarylboronic pinacolate ester $\mathbf{s} 2$ for our mechanism investigations. ${ }^{3}$

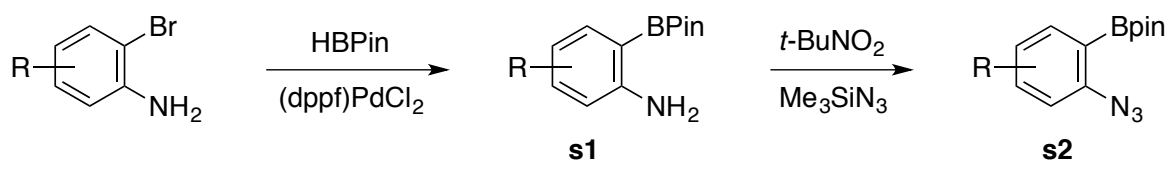

Scheme s1. Synthesis of 2-azidoarylboronic pinacolate ester reagents.

\section{B. Synthesis of 2-Aminoarylboronic Acid Pinacolate Esters.}

\section{General Procedure.}
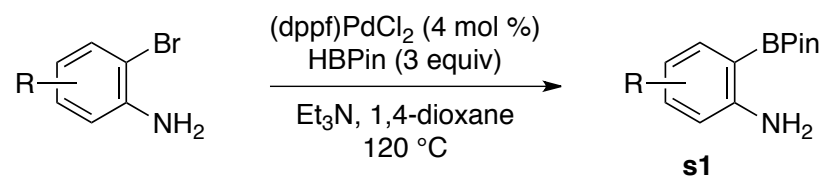

To a mixture of $1.0 \mathrm{~g}$ of 2-bromoaniline (5.8 mmol), $3.22 \mathrm{~mL}$ of $\mathrm{Et}_{3} \mathrm{~N}(23.2 \mathrm{mmol}), 0.208 \mathrm{~g}$ of (dppf)PdCl${ }_{2}(0.25 \mathrm{mmol})$ in $20 \mathrm{~mL}$ of 1,4-dioxane, was added dropwise $2.53 \mathrm{~mL}$ of 4,4,5,5-tetramethyl-1,3,2-dioxaborolane (17.4 mmol). The resultant mixture was refluxed at $120^{\circ} \mathrm{C}$. After $12 \mathrm{~h}$, the mixture was cooled to room temperature and diluted with $20 \mathrm{~mL}$ of $\mathrm{NH}_{4} \mathrm{Cl}$. The phases were separated and the resulting aqueous phase was extracted with an additional $2 \times 20 \mathrm{~mL}$ of $\mathrm{CH}_{2} \mathrm{Cl}_{2}$. The combined organic phases were washed with $1 \times 30 \mathrm{~mL}$ of brine. The resulting organic phase was dried over $\mathrm{Na}_{2} \mathrm{SO}_{4}$, filtered, and the filtrate was concentrated in vacuo. Purification via MPLC afforded the product.

\section{Characterization data for 2 -aminoarylboronic acid pinacolate esters.}

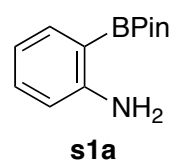

Aniline s1a. ${ }^{2}$ The general procedure was followed using $3.40 \mathrm{~g}$ of 2-bromoaniline $(20.0 \mathrm{mmol}), 8.7 \mathrm{~mL}$ of $4,4,5,5-$ tetramethyl-1,3,2-dioxaborolane $(60.0 \mathrm{mmol}), 0.816 \mathrm{~g}$ of (dppf) $\mathrm{PdCl}_{2}(1.00 \mathrm{mmol})$, and $8.4 \mathrm{~mL}^{-} \mathrm{Et}_{3} \mathrm{~N}(80.0 \mathrm{mmol})$ in $100 \mathrm{~mL}$ of 1,4-dioxane. Purification by MPLC (2:100 - 10:90 EtOAc: hexanes) afforded the product as a light yellow solid (3.02 g, 69\%): $\mathrm{mp} 62-64{ }^{\circ} \mathrm{C}$; the spectral data matched that reported by Driver and co-workers and this compound is also available commercially. ${ }^{2}{ }^{1} \mathrm{H} \mathrm{NMR}\left(500 \mathrm{MHz}, \mathrm{CDCl}_{3}\right) \delta 7.64(\mathrm{~d}, J=7.5 \mathrm{~Hz}, 1 \mathrm{H}), 7.24(\mathrm{t}, J=7.0 \mathrm{~Hz}, 1 \mathrm{H}), 6.70(\mathrm{t}, J$ $=7.0 \mathrm{~Hz}, 1 \mathrm{H}), 6.61(\mathrm{~d}, J=8.5 \mathrm{~Hz}, 1 \mathrm{H}), 4.76(\mathrm{~s}, 2 \mathrm{H}), 1.36(\mathrm{~s}, 12 \mathrm{H}) ;{ }^{13} \mathrm{C} \mathrm{NMR}\left(125 \mathrm{MHz}, \mathrm{CDCl}_{3}\right) \delta 153.7(\mathrm{C}), 136.8(\mathrm{CH})$, $132.8(\mathrm{CH}), 116.9(\mathrm{CH}), 114.8(\mathrm{CH}), 83.5(\mathrm{C}), 25.0\left(\mathrm{CH}_{3}\right)$ only visible peaks; IR (thin film): 3486, 3380, 1624, $1605,1352,1311,1244,1135,1086,847,758,654 \mathrm{~cm}^{-1}$. 


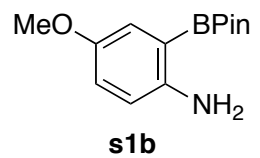

Aniline s1b. ${ }^{4}$ The general procedure was followed using $0.850 \mathrm{~g}$ of 2-bromo-4-methoxyaniline (4.20 mmol), $1.83 \mathrm{~mL}$ of 4,4,5,5-tetramethyl-1,3,2-dioxaborolane (12.6 mmol), $0.170 \mathrm{~g}$ of (dppf) $\mathrm{PdCl}_{2}(0.210 \mathrm{mmol})$, and $2.34 \mathrm{~mL}$ of $\mathrm{Et}_{3} \mathrm{~N}(16.8$ $\mathrm{mmol})$ in $42 \mathrm{~mL}$ of 1,4-dioxane. Purification by MPLC (2:100 - 10:90 EtOAc: hexanes) afforded the product as a brown liquid $(0.670 \mathrm{~g}, 64 \%)$; the spectral data matched that reported by Driver and co-workers: $:{ }^{1} \mathrm{H} \mathrm{NMR}\left(500 \mathrm{MHz}, \mathrm{CDCl}_{3}\right) \delta$ $7.15(\mathrm{~s}, 1 \mathrm{H}), 6.85(\mathrm{dd}, \mathrm{J}=8.5,3.0 \mathrm{~Hz}, 1 \mathrm{H}), 6.57(\mathrm{~d}, \mathrm{~J}=8.5 \mathrm{~Hz}, 1 \mathrm{H}), 4.47(\mathrm{~s}, 2 \mathrm{H}), 3.76(\mathrm{~s}, 3 \mathrm{H}), 1.34(\mathrm{~s}, 12 \mathrm{H}) ;{ }^{13} \mathrm{C} \mathrm{NMR}$ $\left(125 \mathrm{MHz}, \mathrm{CDCl}_{3}\right) \delta 151.4(\mathrm{C}), 148.0(\mathrm{C}), 120.6(\mathrm{CH}), 119.6(\mathrm{CH}), 116.5(\mathrm{CH}), 83.6(\mathrm{C}), 56.0\left(\mathrm{CH}_{3}\right), 25.0\left(\mathrm{CH}_{3}\right)$ only visible peaks; IR (thin film): 3456, 3366, 1494, 1421, 1359, 1304, 1226, 1037, 855, 829, $750 \mathrm{~cm}^{-1}$.

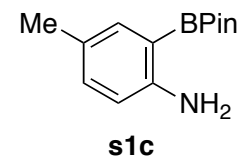

Aniline s1c. ${ }^{4 \mathrm{~b}}$ The general procedure was followed using $0.930 \mathrm{~g}$ of 2-bromo-4-methylaniline $(5.00 \mathrm{mmol}), 2.20 \mathrm{~mL}$ of

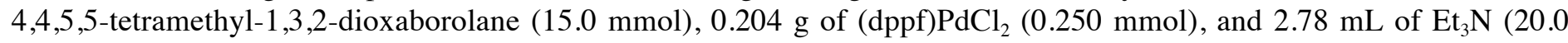
$\mathrm{mmol})$ in $25 \mathrm{~mL}$ of 1,4-dioxane. Purification by MPLC (2:100 - 10:90 EtOAc: hexanes) afforded the product as a light yellow solid $(0.490 \mathrm{~g}, 42 \%)$ : $\mathrm{mp} 60{ }^{\circ} \mathrm{C}$; the spectral data matched that reported by Driver and co-workers: ${ }^{4 \mathrm{~b}}{ }^{1} \mathrm{H}$ NMR $(500$ $\left.\mathrm{MHz} \mathrm{CDCl}_{3}\right) \delta 7.48(\mathrm{~s}, 1 \mathrm{H}), 7.08(\mathrm{~d}, \mathrm{~J}=8.0 \mathrm{~Hz}, 1 \mathrm{H}), 6.57(\mathrm{~d}, \mathrm{~J}=8.0 \mathrm{~Hz}, 1 \mathrm{H}), 4.65(\mathrm{~s}, 2 \mathrm{H}), 2.26(\mathrm{~s}, 3 \mathrm{H}), 1.38(\mathrm{~s}, 12 \mathrm{H})$; ${ }^{13} \mathrm{C} \mathrm{NMR}\left(125 \mathrm{MHz}, \mathrm{CDCl}_{3}\right) \delta 151.5(\mathrm{C}), 136.8(\mathrm{CH}), 133.7(\mathrm{CH}), 125.8(\mathrm{C}), 115.1(\mathrm{CH}), 83.5(\mathrm{C}), 67.1(\mathrm{C}), 25.0\left(\mathrm{CH}_{3}\right)$, $20.3\left(\mathrm{CH}_{3}\right)$; IR (thin film): 3500, 2980, 2244, 1618, 1576, $1496 \mathrm{~cm}^{-1}$.

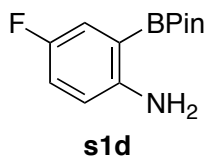

Aniline s1d. ${ }^{5}$ The general procedure was followed using $0.950 \mathrm{~g}$ of 2-bromo-4-fluoroaniline (5.00 mmol), $2.2 \mathrm{~mL}$ of 4,4,5,5-tetramethyl-1,3,2-dioxaborolane (15.0 mmol), $0.204 \mathrm{~g}$ of (dppf) $\mathrm{PdCl}_{2}(0.250 \mathrm{mmol})$, and $2.78 \mathrm{~mL} \mathrm{of} \mathrm{Et}_{3} \mathrm{~N}(20.0$ $\mathrm{mmol})$ in $25 \mathrm{~mL}$ of 1,4-dioxane. Purification by MPLC (2:100 - 10:90 EtOAc: hexanes) afforded the product as a light yellow solid $(0.900 \mathrm{~g}, 76 \%)$; the spectral data matched that reported by Driver and co-workers: ${ }^{5}{ }^{1} \mathrm{H} \mathrm{NMR}(500 \mathrm{MHz}$, $\left.\mathrm{CDCl}_{3}\right) \delta 7.28(\mathrm{dd}, \mathrm{J}=9.0 \mathrm{~Hz}, 3.0 \mathrm{~Hz}, 1 \mathrm{H}), 6.92(\mathrm{dt}, \mathrm{J}=8.5 \mathrm{~Hz}, 3.0 \mathrm{~Hz}, 1 \mathrm{H}), 6.53(\mathrm{dd}, \mathrm{J}=9.0 \mathrm{~Hz}, 4.5 \mathrm{~Hz}, 1 \mathrm{H}), 4.59(\mathrm{~s}$, $2 \mathrm{H}), 1.34(\mathrm{~s}, 12 \mathrm{H}) ;{ }^{13} \mathrm{C} \mathrm{NMR}\left(125 \mathrm{MHz}, \mathrm{CDCl}_{3}\right) \delta 155.2\left(\mathrm{~d}, J_{C F}=233.2 \mathrm{~Hz}, \mathrm{C}\right), 149.8(\mathrm{C}), 121.6\left(\mathrm{~d}, J_{C F}=20.1 \mathrm{~Hz}, \mathrm{CH}\right)$, $119.7\left(\mathrm{~d}, J_{C F}=23.7 \mathrm{~Hz}, \mathrm{CH}\right), 116.0(\mathrm{CH}), 83.9(\mathrm{C}), 24.9\left(\mathrm{CH}_{3}\right)$ only peaks visible; ${ }^{19} \mathrm{~F}$ NMR $\left(282 \mathrm{MHz}, \mathrm{CDCl}_{3}\right) \delta-129.5$; IR (thin film): 3470, 3371, 2975, 2931, 1624, 1492, 1434, 1380, 1347, 1198, 1190, 1135, 1081, 963, $912 \mathrm{~cm}^{-1}$.

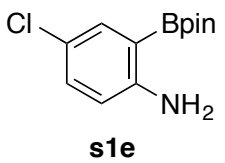

Aniline s1e. The general procedure was followed using $2.06 \mathrm{~g}$ of 2-bromo-4-chloroaniline (10.0 mmol), $4.33 \mathrm{~mL}$ of HBpin (30.0 mmol), $0.701 \mathrm{~g}$ of $\left(\mathrm{PPh}_{3}\right)_{2} \mathrm{PdCl}_{2}(1.00 \mathrm{mmol})$, and $5.60 \mathrm{~mL}$ of Et ${ }_{3} \mathrm{~N}(40.0 \mathrm{mmol})$ in $100 \mathrm{~mL}$ of 1,4-dioxane. Purification by MPLC (0:100 - 20:80 EtOAc: hexanes) afforded the product as a white solid $(2.01 \mathrm{~g}, 79 \%)$. The product has previously been reported by Maleczka, Smith and co-workers: ${ }^{6}{ }^{1} \mathrm{H}$ NMR $\left(500 \mathrm{MHz}, \mathrm{CDCl}_{3}\right) \delta 7.55(\mathrm{~d}, J=2.5 \mathrm{~Hz}$, $1 \mathrm{H}), 7.13(\mathrm{dd}, J=7.5 \mathrm{~Hz}, 2.5 \mathrm{~Hz}, 1 \mathrm{H}), 6.52(\mathrm{~d}, J=7.5 \mathrm{~Hz}, 1 \mathrm{H}), 4.65(\mathrm{br} \mathrm{s}, 2 \mathrm{H}), 1.34(\mathrm{~s}, 12 \mathrm{H}) ;{ }^{13} \mathrm{C} \mathrm{NMR}(125 \mathrm{MHz}$, $\left.\mathrm{CDCl}_{3}\right) \delta 152.1(\mathrm{C}), 135.8(\mathrm{CH}), 132.5(\mathrm{CH}), 121.6(\mathrm{C}), 116.0(\mathrm{CH}), 83.9(\mathrm{C}), 24.9\left(\mathrm{CH}_{3}\right)$ only visible peaks. ATR-FTIR (thin film): $3485,3388,2978,1619,1482,1416,1351 \mathrm{~cm}^{-1}$. 


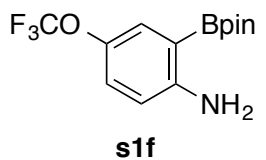

Aniline s1f. ${ }^{7}$ The general procedure for the Pd-catalyzed borylation reaction was followed using $1.28 \mathrm{~g}$ of 2-bromo-4trifluoromethoxyaniline $(5.00 \mathrm{mmol}), 2.20 \mathrm{~mL}$ of 4,4,5,5-tetramethyl-1,3,2-dioxaborolane $(15.0 \mathrm{mmol}), 0.204 \mathrm{~g}$ of (dppf) $\mathrm{PdCl}_{2}(0.250 \mathrm{mmol})$, and $2.78 \mathrm{~mL}$ of $\mathrm{Et}_{3} \mathrm{~N}(20.0 \mathrm{mmol})$ in $25 \mathrm{~mL}$ of 1,4-dioxane. Purification by MPLC $(0: 100-$ 20:80 EtOAc: hexanes) afforded the product as a light yellow solid $(0.970 \mathrm{~g}, 59 \%)$ : $\mathrm{mp} 63-67{ }^{\circ} \mathrm{C}$; the product has previously been reported by Driver and co-workers: ${ }^{7} \mathrm{H}$ NMR $\left(500 \mathrm{MHz}, \mathrm{CDCl}_{3}\right) \delta 7.45(\mathrm{~s}, 1 \mathrm{H}), 7.06(\mathrm{~d}, J=8.5 \mathrm{~Hz}, 1 \mathrm{H})$, $6.54(\mathrm{~d}, J=9.0 \mathrm{~Hz}, 1 \mathrm{H}), 4.80(\mathrm{~s}, 2 \mathrm{H}), 1.34(\mathrm{~s}, 12 \mathrm{H}) ;{ }^{13} \mathrm{C} \mathrm{NMR}\left(125 \mathrm{MHz}, \mathrm{CDCl}_{3}\right) \delta 152.5(\mathrm{C}), 140.0(\mathrm{C}), 129.0(\mathrm{CH})$, $126.0(\mathrm{CH}), 122.8\left(\mathrm{q}, J_{C F}=253.5 \mathrm{~Hz}, \mathrm{C}\right), 115.5(\mathrm{CH}), 84.0(\mathrm{C}), 24.9\left(\mathrm{CH}_{3}\right)$, only visible peaks; ${ }^{19} \mathrm{~F}$ NMR $(282 \mathrm{MHz}$, $\mathrm{CDCl}_{3}$ ) $\delta$-58.8; IR (thin film) 3477, 3374, 2992, 2980, 1627, 1492, 1436, 1532, 1211, 1163, 1094, 965, 852, $825 \mathrm{~cm}^{-1}$.

\section{Synthesis of 2-Azidoarylboronic Acid Pinacolate Esters.}

1. General Procedure.

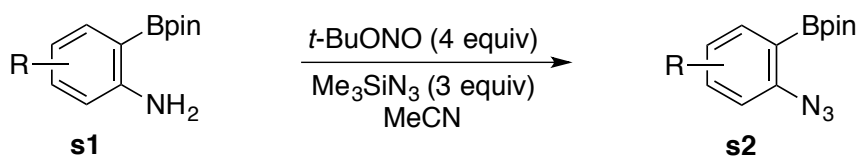

To a cooled solution $\left(0{ }^{\circ} \mathrm{C}\right)$ of aniline in $\mathrm{MeCN}(0.2 \mathrm{M})$ was added dropwise $t$-BuONO (4.0 equiv) and $\mathrm{Me}_{3} \mathrm{SiN}_{3}(3.0$ equiv). The resulting solution was warmed to room temperature. After $1.5 \mathrm{~h}$, the reaction mixture was concentrated in vacuo. Purification of the residue by MPLC (0:100 - 5:95 EtOAc: hexanes) afforded the 2-azidoarylboronic acid pinacolate ester.

\section{Characterization data for 2-azidoarylboronic acid pinacolate esters.}

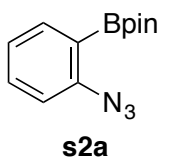

2-Azidophenylboronic acid pinacolate ester s2a. ${ }^{7}$ The general procedure was followed by using $1.60 \mathrm{~g}$ of aniline s1a (7.30 mmol), $3.47 \mathrm{~mL}$ of $t$-BuONO (29.2 mmol) and $2.90 \mathrm{~mL}$ of $\mathrm{Me}_{3} \mathrm{SiN}_{3}(21.9 \mathrm{mmol})$ in $36 \mathrm{~mL}$ of MeCN. Purification by MPLC (0:100 - 10:90 EtOAc:hexanes) afforded the product as a yellow oil $(1.091 \mathrm{~g}, 61 \%)$; the spectral data matched that reported by Driver and co-workers: ${ }^{7} \mathrm{H}$ NMR $\left(500 \mathrm{MHz}, \mathrm{CDCl}_{3}\right) \delta 7.73(\mathrm{~d}, J=7.5 \mathrm{~Hz}, 1 \mathrm{H}), 7.45(\mathrm{t}, J=7.0 \mathrm{~Hz}, 1 \mathrm{H})$, $7.15-7.10(\mathrm{~m}, 2 \mathrm{H}), 1.36(\mathrm{~s}, 12 \mathrm{H}) ;{ }^{13} \mathrm{C}$ NMR $\left(125 \mathrm{MHz}, \mathrm{CDCl}_{3}\right) \delta 144.8(\mathrm{C}), 137.0(\mathrm{CH}), 132.4(\mathrm{CH}), 124.2(\mathrm{CH}), 118.2$ $(\mathrm{CH}), 84.0(\mathrm{C}), 24.8\left(\mathrm{CH}_{3}\right)$ only visible signals. ATR-FTIR (thin film): 2976, 2112, 2076, 1594, 1572, 1487, 1432, 1351, $1316,1279,1143,1110,1058,1036,836,747 \mathrm{~cm}^{-1}$.

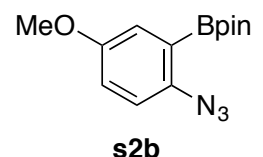

2-Azido-5-methoxyphenylboronic acid pinacolate ester s2b. ${ }^{7}$ The general procedure was followed by using $0.550 \mathrm{~g}$ of aniline s1b $(2.0 \mathrm{mmol}), 0.95 \mathrm{~mL}$ of $t-\mathrm{BuNO}_{2}(8.0 \mathrm{mmol})$ and $0.80 \mathrm{~mL}$ of $\mathrm{Me}_{3} \mathrm{SiN}_{3}(6.0 \mathrm{mmol})$ in $10 \mathrm{~mL} \mathrm{of} \mathrm{MeCN}$. Purification by MPLC (0:100 - 10:90 EtOAc:hexanes) afforded the product, a yellow solid (0.396 g, 72\%). The spectral data matched that reported by Driver and co-workers: ${ }^{7}{ }^{1} \mathrm{H}$ NMR $\left(500 \mathrm{MHz}, \mathrm{CDCl}_{3}\right) \delta 7.24(\mathrm{~d}, J=2.5 \mathrm{~Hz}, 1 \mathrm{H}), 7.07(\mathrm{~d}, J$ 
$=9.0 \mathrm{~Hz}, 1 \mathrm{H}), 6.98(\mathrm{dd}, J=8.5 \mathrm{~Hz}, 3.0 \mathrm{~Hz}, 1 \mathrm{H}), 3.81(\mathrm{~s}, 3 \mathrm{H}), 1.36(\mathrm{~s}, 12 \mathrm{H}) ;{ }^{13} \mathrm{C} \mathrm{NMR}\left(125 \mathrm{MHz}, \mathrm{CDCl}_{3}\right) \delta 156.3(\mathrm{C})$, $137.3(\mathrm{C}), 120.9(\mathrm{CH}), 119.7(\mathrm{CH}), 118.6(\mathrm{CH}), 80.1(\mathrm{C}), 55.6\left(\mathrm{CH}_{3}\right), 24.8\left(\mathrm{CH}_{3}\right)$ only visible peaks. ATR-FTIR (thin film): 2976, 2934, 2118, 1484, 1409, 1341, 1231, 1143, 1052, 906, $724 \mathrm{~cm}^{-1}$. HRMS (ESI) m/z calculated for $\mathrm{C}_{13} \mathrm{H}_{18} \mathrm{BN}_{3} \mathrm{O}_{3} \mathrm{Na}(\mathrm{M}+\mathrm{Na})^{+}:$298.1339, found: 298.1345.

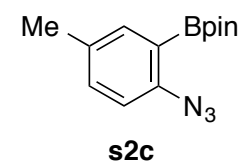

2-Azido-5-methylphenylboronic acid pinacolate ester s2c. ${ }^{7}$ The general procedure was followed by using $0.518 \mathrm{~g}$ of aniline s1c $(2.00 \mathrm{mmol}), 0.95 \mathrm{~mL}$ of $t$-BuONO $(8.0 \mathrm{mmol})$ and $0.80 \mathrm{~mL}$ of $\mathrm{Me}_{3} \mathrm{SiN}_{3}(6.0 \mathrm{mmol})$ in $10 \mathrm{~mL}$ of $\mathrm{MeCN}$. Purification by MPLC (0:100 - 10:90 EtOAc:hexanes) afforded the product as an orange oil (0.253 g, 49\%); the spectral data matched that reported by Driver and co-workers: ${ }^{7}{ }^{1} \mathrm{H}$ NMR $\left(500 \mathrm{MHz}, \mathrm{CDCl}_{3}\right) \delta 7.54(\mathrm{~d}, J=1.5 \mathrm{~Hz}, 1 \mathrm{H}), 7.26(\mathrm{dd}, J$ $=8.5 \mathrm{~Hz}, 1.5 \mathrm{~Hz}, 1 \mathrm{H}), 7.05(\mathrm{~d}, J=8.0 \mathrm{~Hz}, 1 \mathrm{H}), 2.31(\mathrm{~s}, 3 \mathrm{H}), 1.36(\mathrm{~s}, 12 \mathrm{H}) ;{ }^{13} \mathrm{C} \mathrm{NMR}\left(125 \mathrm{MHz}, \mathrm{CDCl}_{3}\right) \delta 142.1(\mathrm{C})$, $137.4(\mathrm{CH}), 133.8(\mathrm{C}), 133.0(\mathrm{CH}), 118.2(\mathrm{CH}), 84.0(\mathrm{C}), 24.8\left(\mathrm{CH}_{3}\right), 20.7\left(\mathrm{CH}_{3}\right)$ only visible peaks. ATR-FTIR (thin film): 2979, 2924, 2118, 2092, 1579, 1487, 1400, 1345, 1312, 1269, 1146, 906, $730 \mathrm{~cm}^{-1}$.

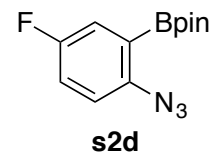

2-Azido-5-fluorophenylboronic acid pinacolate ester s2d. ${ }^{7}$ The general procedure was followed by using $0.526 \mathrm{~g}$ of aniline s1d $(2.0 \mathrm{mmol}), 0.95 \mathrm{~mL}$ of $t-\mathrm{BuNO}_{2}(8.0 \mathrm{mmol})$ and $0.80 \mathrm{~mL}$ of $\mathrm{Me}_{3} \mathrm{SiN}_{3}(6.0 \mathrm{mmol})$ in $10 \mathrm{~mL}$ of $\mathrm{MeCN}$. Purification by MPLC (0:100 - 10:90 EtOAc:hexanes) afforded the product, a red oil $(0.373 \mathrm{~g}, 71 \%)$. The spectral data matched that reported by Driver and co-workers: ${ }^{7}{ }^{1} \mathrm{NMR}\left(500 \mathrm{MHz}, \mathrm{CDCl}_{3}\right) \delta 7.39(\mathrm{dd}, J=8.5 \mathrm{~Hz}, 3.0 \mathrm{~Hz}, 1 \mathrm{H}), 7.10$ (ddd, $J=10.5 \mathrm{~Hz}, 7.5 \mathrm{~Hz}, 3.0 \mathrm{~Hz}, 1 \mathrm{H}), 7.05(\mathrm{dd}, J=8.5 \mathrm{~Hz}, 4.0 \mathrm{~Hz}, 1 \mathrm{H}), 1.34(\mathrm{~s}, 12 \mathrm{H}) ;{ }^{13} \mathrm{C} \mathrm{NMR}\left(125 \mathrm{MHz}, \mathrm{CDCl}_{3}\right) \delta$ $159.4\left(\mathrm{~d}, J_{C F}=242.9 \mathrm{~Hz}, \mathrm{C}\right), 140.5(\mathrm{C}), 123.0\left(\mathrm{~d}, J_{C F}=20.5 \mathrm{~Hz}, \mathrm{CH}\right), 119.9\left(\mathrm{~d}, J_{C F}=7.5 \mathrm{~Hz}, 1 \mathrm{H}\right), 119.1\left(\mathrm{~d}, J_{C F}=23.5 \mathrm{~Hz}\right.$, $\mathrm{CH}), 84.3(\mathrm{C}), 24.8\left(\mathrm{CH}_{3}\right)$, only visible peaks. HRMS (EI) $\mathrm{m} / z$ calcd for $\mathrm{C}_{12} \mathrm{H}_{15} \mathrm{BN}_{3} \mathrm{O}_{2} \mathrm{~F}[\mathrm{M}]^{+}: 263.1241$, found 263.1233. ATR-FTIR (thin film): 2989, 2121, 2092, 1485, 1416, 1319, 1200, 1143, 1126, 966, 916, 807, $763 \mathrm{~cm}^{-1}$.

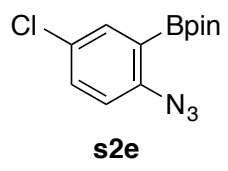

2-Azido-5-chlorophenylboronic acid pinacol ester s2e. ${ }^{7}$ The general procedure was followed by using $0.13 \mathrm{~g}$ of 2 amino-5-chlorophenylboronic acid pinacolate ester s1e $(0.52 \mathrm{mmol}), 0.27 \mathrm{~mL}$ of $t$-BuONO $(2.1 \mathrm{mmol}), 0.22 \mathrm{~mL}$ of $\mathrm{Me}_{3} \mathrm{SiN}_{3}(1.6 \mathrm{mmol})$ and $10 \mathrm{~mL}$ of MeCN. Purification by MPLC (1:99 - 5:95 EtOAc: hexanes) afforded the product, an orange oil $(0.06 \mathrm{~g}, 42 \%)$; the spectral data matched that reported by Driver and co-workers: ${ }^{7}{ }^{1} \mathrm{H} \mathrm{NMR}(500 \mathrm{MHz}, \mathrm{CDCl} 3)$ $\delta 7.69(\mathrm{~s}, 1 \mathrm{H}), 7.39(\mathrm{~d}, J=8.4 \mathrm{~Hz}, 1 \mathrm{H}), 7.06(\mathrm{~d}, J=8.5 \mathrm{~Hz}, 1 \mathrm{H}), 1.35(\mathrm{~s}, 12 \mathrm{H}) ;{ }^{13} \mathrm{C} \mathrm{NMR}\left(125 \mathrm{MHz}, \mathrm{CDCl}_{3}\right) \delta 143.3(\mathrm{C})$, $136.6(\mathrm{CH}), 132.1(\mathrm{CH}), 129.8(\mathrm{C}), 119.7(\mathrm{CH}), 84.4(\mathrm{C}), 24.8\left(\mathrm{CH}_{3}\right)$, only visible peaks. ATR-FTIR (thin film): 2980 , $2119,2085,1480,1402,1334 \mathrm{~cm}^{-1}$.

\section{Synthesis of Trisubstituted Styryl Anilines.}

\section{A. Substrate Synthesis Overview.}

The trisubstituted styryl azides were constructed from cyclobutanone and 2-iodoaniline following the process outlined in equation s3. Addition of the Grignard acetylide to cyclobutanone afforded cyclobutanol s3a, which was subjected to the Heck reaction conditions reported by Larock and Reddy to furnish the trisubstituted styrene $\mathbf{s} 3 \mathbf{b}{ }^{8}{ }^{8}$ 


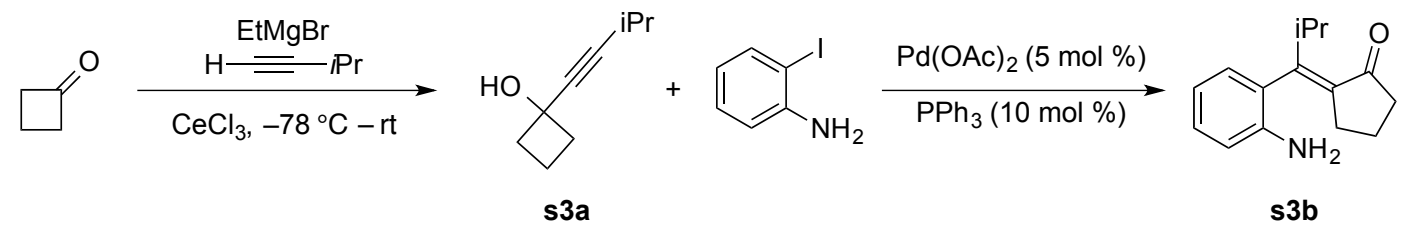

\section{B. Synthesis of Cyclobutanol.}
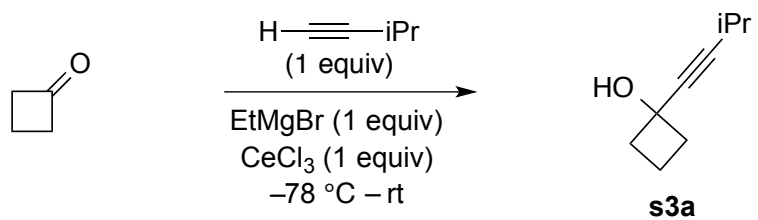

$(s 4)$

1-(3-Methylbut-1-yn-1-yl)cyclobutan-1-ol s3a. ${ }^{9}$ A $100 \mathrm{~mL}$ flame-dried round bottom flask was charged with $14.6 \mathrm{~mL}$ of a 1.0 M solution of ethyl magnesium bromide in THF (14.6 mmol). 3-Methyl-1-butyne (1.51 mL, $14.6 \mathrm{mmol})$ was then added dropwise at room temperature. After $2 \mathrm{~h}$, the crude reaction mixture was then added slowly to a $100 \mathrm{~mL}$ Schlenk flask charged with $3.74 \mathrm{~g}$ of cerium chloride $(14.6 \mathrm{mmol})$ in $30 \mathrm{~mL}$ of $\mathrm{THF}$ at $-78{ }^{\circ} \mathrm{C}$. After $1 \mathrm{~h}$, a solution of $0.787 \mathrm{~g}$ of cyclobutanone $(11.2 \mathrm{mmol})$ in $10 \mathrm{~mL}$ of THF was added dropwise. The resulting reaction mixture was allowed to warm to room temperature. After $6 \mathrm{~h}$, the reactives in the heterogeneous mixture were quenched with water, and the resulting mixture was extracted with $3 \times 20 \mathrm{~mL}_{\text {of }} \mathrm{Et}_{2} \mathrm{O}$. The combined organic phases were washed with $1 \times 25 \mathrm{~mL}_{\text {of }} \mathrm{NH}_{4} \mathrm{Cl}, 1 \times$ $25 \mathrm{~mL}$ of distilled water and $1 \times 25 \mathrm{~mL}$ of brine. The organic phase was dried over $\mathrm{MgSO}_{4}$, and the heterogeneous mixture was filtered. The filtrate was concentrated in vacuo to afford an orange oil, which was purified by flash chromatography (10:90 EtOAc:hexanes) to afford the product s3a $(1.12 \mathrm{~g}, 72 \%)$ : ${ }^{1} \mathrm{H}$ NMR $\left(500 \mathrm{MHz}, \mathrm{CDCl}_{3}\right) \delta 2.60$ (septet, $J=7.0 \mathrm{~Hz}, 1 \mathrm{H}), 2.39-2.35(\mathrm{~m}, 2 \mathrm{H}), 2.25-2.19(\mathrm{~m}, 2 \mathrm{H}), 2.09(\mathrm{~d}, J=1.5 \mathrm{~Hz}, 1 \mathrm{H}), 1.81-1.75(\mathrm{~m}, 2 \mathrm{H}), 1.17(\mathrm{~d}, J$ $=6.5 \mathrm{~Hz}, 6 \mathrm{H}$ ); ATR-FTIR (thin film): 3331, 2969, 2940, 2871, 1456, 1320, 1244, $1120 \mathrm{~cm}^{-1}$. The cyclobutanol was submitted to the Heck reaction without additional characterization.

\section{Synthesis of Aniline.}
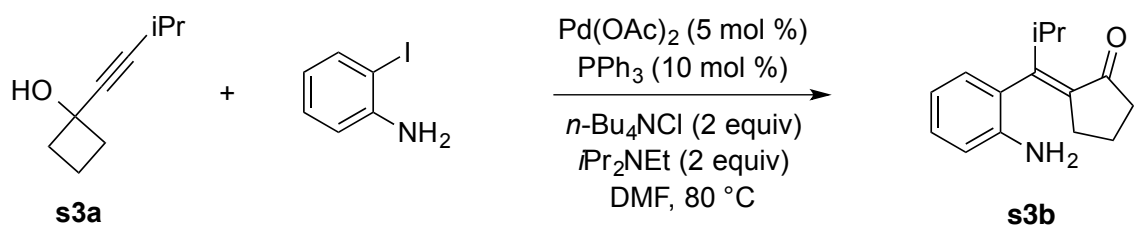

(s5)

Aniline s3b. In a flame-dried $100 \mathrm{~mL}$ round bottom flask was dissolved $1.00 \mathrm{~g}$ of 1-(3-methylbut-1-yn-1-yl)cyclobutan1-ol s3a $(7.24 \mathrm{mmol}), 1.59 \mathrm{~g}$ of 2-iodoaniline $(7.24 \mathrm{mmol}), 4.02 \mathrm{~g}$ of $n$ - $\mathrm{Bu} \mathrm{u}_{4} \mathrm{NCl}(14.5 \mathrm{mmol}), 2.52 \mathrm{~mL}$ of $\mathrm{iPr}_{2} \mathrm{NEt}(14.5$ $\mathrm{mmol}), 0.380 \mathrm{~g}$ of $\mathrm{PPh}_{3}(1.45 \mathrm{mmol})$ and $0.325 \mathrm{~g}$ of $\mathrm{Pd}(\mathrm{OAc})_{2}(0.1 .45 \mathrm{mmol})$ in $28 \mathrm{~mL}$ of DMF. The flask was then degassed, flushed with nitrogen, placed in an $80{ }^{\circ} \mathrm{C}$ oil bath. After 12 hours, the heterogeneous mixture was diluted with $1 \times$ $50 \mathrm{~mL}$ of water, and the mixture was extracted with $3 \times 50 \mathrm{~mL}$ of $\mathrm{CH}_{2} \mathrm{Cl}_{2}$. The combined organic phases were washed with $2 \times 25 \mathrm{~mL}$ of distilled water and $1 \times 25 \mathrm{~mL}$ of brine. The resulting organic phase was dried over $\mathrm{Na}_{2} \mathrm{SO}_{4}$, and the heterogeneous mixture was filtered. The filtrate was concentrated in vacuo to yield a dark red oil. The product was purified by flash chromatography (10:90 EtOAc:hexanes) affording the product as an orange oil (1.07 g, 64\%). We found that cyclopentanones contained inseparable impurities. These compounds were carried on to the azidation step without further purification. After this step, these impurities could be removed from the styryl azides. 


\section{Preparation of Vinyl Triflates.}

\section{A. Preparation of $\beta$-Ketoesters.}

\section{General Procedures.}

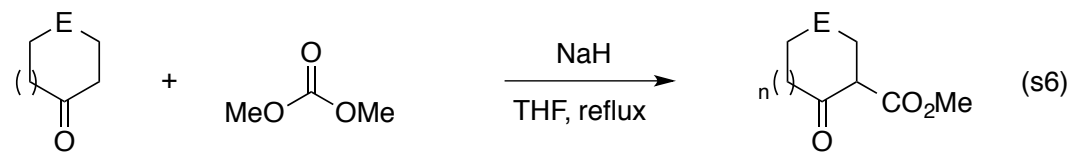

Method A: To sodium hydride (60\% oil dispersion, 4 equiv) was added a solution of dimethyl carbonate ( 3 equiv) in dry THF $(1 \mathrm{M})$. The mixture was stirred at reflux, and then a solution of ketone (1 equiv) in dry THF (2M) was added dropwise to the mixture using a syringe pump. After $2-12$ hours, the reaction mixture was cooled using an ice bath. After $20 \mathrm{~min}$, the reaction mixture was diluted with ether. The mixture was hydrolyzed by the slow addition of a $1 \mathrm{M}$ aqueous $\mathrm{NH}_{4} \mathrm{Cl}$ solution, then poured into brine and extracted with diethyl ether $(4 \times 30 \mathrm{~mL})$. The combined organic layers were dried over $\mathrm{Na}_{2} \mathrm{SO}_{4}$, filtered, and the filtrate was concentrated in vacuo. Purification of the residue by MPLC afforded the product.

Method B: To a solution of ketone (1 equiv) in dry THF (2M) was slowly added sodium hydride (60\% oil dispersion, 2.5 equiv). After $30 \mathrm{~min}$, a solution of dimethyl carbonate (2.5 equiv) in dry THF ( $1 \mathrm{M})$ was added dropwise and the solution was stirred at reflux. After $15 \mathrm{~h}$, the reaction mixture was cooled using an ice bath. After 20 min, the reaction mixture was diluted with ether. The mixture was hydrolyzed by the slow addition of $1 \mathrm{M}$ aqueous solution, then poured into brine and extracted with diethyl ether $(4 \times 30 \mathrm{~mL})$. The combined organic layers were dried over $\mathrm{Na}_{2} \mathrm{SO}_{4}$, filtered, and the filtrate was concentrated in vacuo. Purification of the residue by MPLC afforded the product.

\section{Charecterization data.}

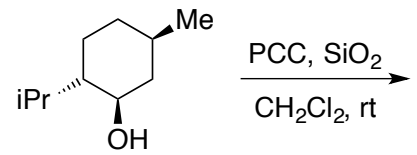<smiles>CC1CC[C@H](C(C)C)C(=O)C1</smiles>

(-)-Menthone

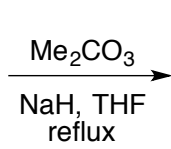

reflux<smiles>CC(=O)C1C(=O)[C@H](C(C)C)CCC1C(C)C</smiles>

s4a (s7)

(-)-Menthone. ${ }^{10}$ Pyridinium chlorochromate $(4.85 \mathrm{~g}, 22.5 \mathrm{mmol})$ was ground with silica gel $(4.85 \mathrm{~g}, 70-230 \mathrm{mesh})$. The resulting mixture was suspended in a round-bottom flask containing $50 \mathrm{~mL}$ of methylene chloride. To the resulting orange suspension was added a solution of (-)-menthol $(2.34 \mathrm{~g}, 15.0 \mathrm{mmol})$ in $100 \mathrm{~mL}$ of methylene chloride while stirring at room temperature. After $90 \mathrm{~min}$, the resulting dark brown suspension was diluted with $120 \mathrm{~mL}$ of diethyl ether and filtered through a Buchner funnel layered with Celite and silica gel. The filtrate was concentrated under reduced pressure and low temperature to give crude (-)-menthone as a colorless oil $(2.40 \mathrm{~g}, 100 \%)$. The crude product was used for the next step without further purification or characterization. ATR-FTIR (thin film): 2954, 2926, 2870, 1707, 1456, $1366,1201 \mathrm{~cm}^{-1}$.

Methyl (3S,6R)-3-isopropyl-6-methyl-2-oxocyclohexane-1-carboxylate s4a. Method A was followed using $0.730 \mathrm{~g}$ of $\mathrm{NaH}(18.2 \mathrm{mmol}), 1.15 \mathrm{~mL}$ of dimethylcarbonate $(13.7 \mathrm{mmol})$, and $0.704 \mathrm{~g}$ of $(-)$-menthone $(4.56 \mathrm{mmol})$ in $15 \mathrm{~mL} \mathrm{of}$ THF. Purification by MPLC (2:98 to 10:90 EtOAc: hexane) afforded the product, a colorless oil, as a 2:1 mixture of diastereomers $(0.913 \mathrm{~g}, 94 \%)$. $\beta$-Ketoester s4a was previously reported by Yamamoto and co-workers: ${ }^{11}{ }^{1} \mathrm{H}$ NMR (500 $\left.\mathrm{MHz} \mathrm{CDCl}_{3}\right) \delta 3.61(\mathrm{~s}, 3 \mathrm{H}), 3.01(\mathrm{~d}, J=10.5 \mathrm{~Hz}, 0.5 \mathrm{H}), 2.56(\mathrm{~m}, 0.28 \mathrm{H}), 2.31-1.83(\mathrm{~m}, 3.84 \mathrm{H}), 1.66-1.59(\mathrm{~m}, 1 \mathrm{H})$, $1.44-1.40(\mathrm{~m}, 2 \mathrm{H}), 0.89(\mathrm{~d}, J=6.5 \mathrm{~Hz}, 3 \mathrm{H}), 0.82(\mathrm{~d}, J=6.0 \mathrm{~Hz}, 3 \mathrm{H}), 0.74(\mathrm{t}, J=6.0 \mathrm{~Hz}, 3 \mathrm{H})$; characteristic data for minor diastereomer: ${ }^{1} \mathrm{H}$ NMR $\left(500 \mathrm{MHz}, \mathrm{CDCl}_{3}\right) \delta 3.62(\mathrm{~s}, 3 \mathrm{H}), 2.94(\mathrm{~d}, J=12.5 \mathrm{~Hz}, 0.45 \mathrm{H}), 0.92(\mathrm{~d}, J=7.0 \mathrm{~Hz}, 3 \mathrm{H})$, $0.78(\mathrm{~d}, J=6.0 \mathrm{~Hz}, 3 \mathrm{H}), 0.68(\mathrm{~d}, J=6.5 \mathrm{~Hz}, 3 \mathrm{H})$. ATR-FTIR (thin film): 2958, 2871, 1764, 1709, 1643, 1607, 1436, $1231,1196 \mathrm{~cm}^{-1}$. 


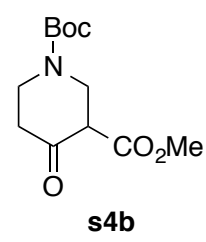

1-tert-Butyl-3-methyl-4-oxopiperidine-1,3-dicarboxylate s4b. ${ }^{12}$ Method B was followed using $1.50 \mathrm{~g}$ of $\mathrm{NaH}(37.5$ $\mathrm{mmol}), 3.2 \mathrm{~mL}$ of dimethylcarbonate $(37.5 \mathrm{mmol})$, and $3.0 \mathrm{~g}$ of 1-Boc-4-piperidone $(15 \mathrm{mmol})$ in $50 \mathrm{~mL}$ of THF. Purification by MPLC (2:98 to 10:90 EtOAc: hexane) afforded the product, a colorless oil, as a mixture of rotamers (2.81 $\mathrm{g}, 74 \%)$; The spectral data matched that reported by Liu and co-workers: ${ }^{12}{ }^{1} \mathrm{H} \mathrm{NMR}\left(500 \mathrm{MHz}, \mathrm{CDCl}_{3}\right) \delta 3.98(\mathrm{~s}, 2 \mathrm{H})$, $3.71(\mathrm{~s}, 3 \mathrm{H}), 3.49(\mathrm{t}, J=5.1 \mathrm{~Hz}, 2 \mathrm{H}), 2.29(\mathrm{~s}, 2 \mathrm{H}), 1.40(\mathrm{~s}, 9 \mathrm{H}) ;{ }^{13} \mathrm{C} \mathrm{NMR}\left(125 \mathrm{MHz}, \mathrm{CDCl}_{3}\right) \delta 202.5(\mathrm{C}), 171.0(\mathrm{C})$, $154.5(\mathrm{C}), 80.0(\mathrm{C}), 60.3\left(\mathrm{CH}_{2}\right), 56.3(\mathrm{CH}), 53.4\left(\mathrm{CH}_{2}\right), 51.5\left(\mathrm{CH}_{3}\right), 28.8\left(\mathrm{CH}_{2}\right), 28.3\left(\mathrm{CH}_{3}\right)$. ATR-FTIR (thin film): 2978, $1691,1661,1619,1226 \mathrm{~cm}^{-1}$.
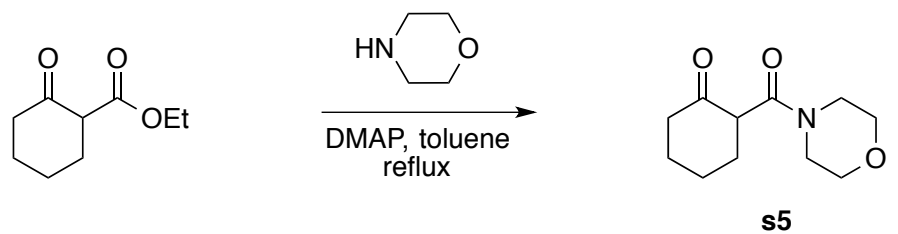

(s8)

Morpholyl-2-oxocyclohexanecarboxamide s5. ${ }^{13}$ To a solution of ethyl 2-oxocyclopentane carboxylate $(1.00 \mathrm{~mL}, 6.25$ mmol) in toluene was added DMAP $(0.229 \mathrm{~g}, 1.87 \mathrm{mmol})$ and morpholine $(5.39 \mathrm{~mL}, 62.5 \mathrm{mmol})$. The mixture was heated to reflux. After $48 \mathrm{~h}$, the reaction mixture was cooled to room temperature and concentrated in vacuo. The resulting residue was purified by MPLC (0:100 - 20:80 EtOAc: hexanes) to afford the product as an orange solid (0.855 g, $65 \%)$. The spectral data matched that reported by Pete and co-workers: ${ }^{12}{ }^{1} \mathrm{H}$ NMR $\left(500 \mathrm{MHz}, \mathrm{CDCl}_{3}\right) \delta 3.77-3.85(1 \mathrm{H}$, $\mathrm{m}), 3.43-3.69(6 \mathrm{H}, \mathrm{m}), 3.29-3.33(2 \mathrm{H}, \mathrm{m}), 2.48-2.58(1 \mathrm{H}, \mathrm{m}), 1.95-2.39(5 \mathrm{H}, \mathrm{m}), 1.64-1.87(2 \mathrm{H}, \mathrm{m})$. ATR-FTIR (thin film): $3300-3600,2930,2859,1707,1634,1439,1269,1239 \mathrm{~cm}^{-1}$. The carboxamide was submitted to the next step without additional characterization.

The desired $\beta$-ketoester s6 was prepared by the method used by Sorensen and co-workers according to Scheme s2. ${ }^{14}$

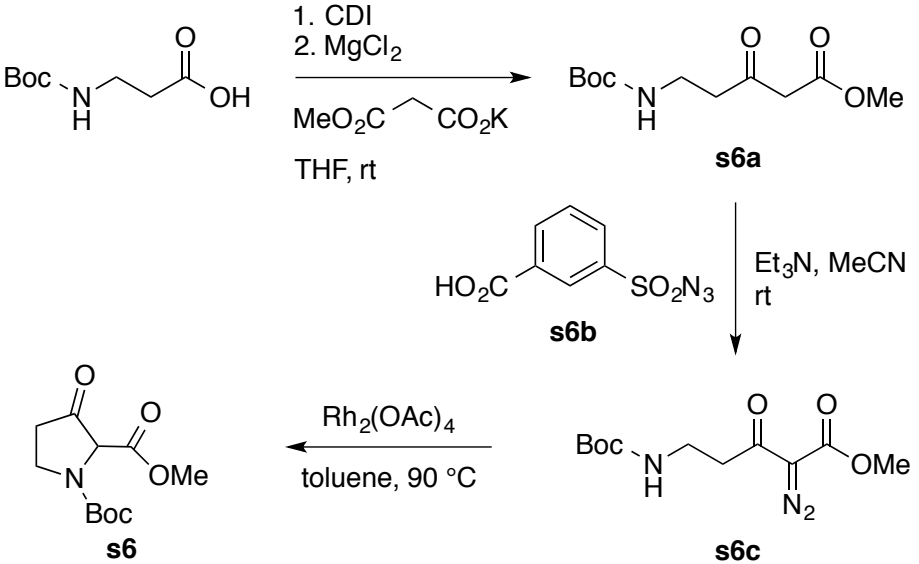

Scheme s2. Synthesis of 1-(tert-butyl)-2-methyl-3-oxopyrrolidine-1,2-dicarboxyate s6. 


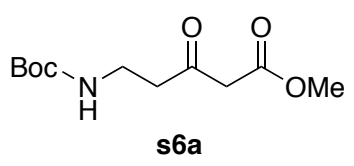

$\boldsymbol{\beta}$-Keto ester s6a. ${ }^{13}$ Under inert atmosphere, solid $N$-Boc- $\beta$-alanine $(2.37 \mathrm{~g}, 12.5 \mathrm{mmol})$ was added in one portion to a suspension of 1,1'-carbonyldiimidazole $(2.43 \mathrm{~g}, 15.0 \mathrm{mmol})$ in $30 \mathrm{~mL}$ of THF in a $100 \mathrm{~mL}$ round bottom flask using a powder funnel. Over the course of $1 \mathrm{~h}$, the resulting suspension was stirred; effervescence occurred and the reaction mixture became a homogeneous, yellow solution. After an additional $3 \mathrm{~h}$, a mixture of $\mathrm{MgCl}_{2}(325 \mathrm{mesh}, 1.19 \mathrm{~g}, 12.5$ mmol) and methyl potassium malonate $(3.90 \mathrm{~g}, 25.0 \mathrm{mmol})$ was added. The resulting suspension was vigorously stirred. After $15 \mathrm{~h}$, the reaction mixture was quenched with $25 \mathrm{~mL}$ of water and $15 \mathrm{~mL}$ of a $1 \mathrm{M}$ aqueous solution of $\mathrm{HCl}$. The resulting yellow, biphasic solution (aqueous layer $\mathrm{pH}$ approx 7) was poured into a $250 \mathrm{~mL}$ separatory funnel, followed by $75 \mathrm{~mL}$ of ethyl acetate. The aqueous layer was removed and the organic layer was washed with $15 \mathrm{~mL}$ of $1 \mathrm{M} \mathrm{HCl}$ and $15 \mathrm{~mL}$ of brine. The combined organic layers were dried over $\mathrm{Na}_{2} \mathrm{SO}_{4}$, filtered and the resulting filtrate was concentrated in vacuo to give yellow oil (3.00 g, 97\%), which was used in the subsequent step without further purification.

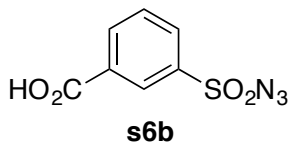

3-(Azidosulfonyl)benzoic acid $\mathbf{s 6 b} .^{13}$ To a $250 \mathrm{~mL}$ round bottom flask was added 3-(chlorosulfonyl)benzoic acid (2.00 g, $9.06 \mathrm{mmol})$ and $36 \mathrm{~mL}$ of acetone. To the resulting light brown solution was added a solution of sodium azide $(0.736 \mathrm{~g}$, $11.3 \mathrm{mmol}$ ) in $6 \mathrm{~mL}$ of water. After $2 \mathrm{~h}$, the reaction mixture was concentrated in vacuo to afford a brown residue. Approximately $50 \mathrm{~mL}$ of water was added to the flask, followed by a few milliliters of concentrated $\mathrm{HCl}$ (solution $\mathrm{pH}<2$ ) to induce precipitation. The precipitate was collected in a sintered glass funnel, rinsed well with water and three portions of hexanes in the funnel. The resulting residue was dried by pulling air through the funnel for $1 \mathrm{~h}$ followed by drying under high vacuum overnight to give the desired sulfonyl azide as an off-white, powdery solid (1.93 g, 80\%); The spectral data matched that reported by Sorensen and co-workers: ${ }^{13}{ }^{1} \mathrm{H}$ NMR $\left(500 \mathrm{MHz}, \mathrm{CDCl}_{3}\right) \delta 1 \mathrm{H}\left(500 \mathrm{MHz}, \mathrm{CDCl}_{3} / \mathrm{DMSO}-\right.$ $\left.\mathrm{d}_{6}\right) \mathrm{d} 11.41(\mathrm{br} \mathrm{s}, 1 \mathrm{H}), 8.58(\mathrm{t}, J=1.6 \mathrm{~Hz}, 1 \mathrm{H}), 8.35(\mathrm{ddd}, J=7.8,1.5,1.3 \mathrm{~Hz}, 1 \mathrm{H}), 8.07(\mathrm{ddd}, J=7.9,2.0,1.2 \mathrm{~Hz}, 1 \mathrm{H})$, 7.67 (t, $J=7.9 \mathrm{~Hz}, 1 \mathrm{H}$ ). ATR-FTIR (thin film): 3080-2550, 2135, 1913, 1712, 1374, 1265, $1177 \mathrm{~cm}^{-1}$. The sulfonyl azide was used in the next step without addition purification or characterization.

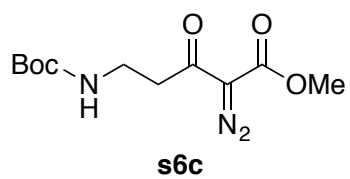

Diazo $\beta$-keto ester s6c. ${ }^{13}$ Under inert atmosphere, sulfonyl azide $\mathbf{s} 6 \mathbf{b}(1.09 \mathrm{~g}, 4.80 \mathrm{mmol})$ was added in one portion to a $\beta$ keto ester s6a $(1.07 \mathrm{~g}, 4.36 \mathrm{mmol})$ in $20 \mathrm{~mL}$ of acetonitrile. Triethylamine $(18.3 \mathrm{~mL}, 13.1 \mathrm{mmol})$ was added dropwise using a pressure equalizing addition funnel over $30 \mathrm{~min}$. After an additional $1 \mathrm{~h}$, the yellow-orange solution was concentrated in vacuo. To the resulting residue was added $75 \mathrm{~mL}$ of 1:1 diethyl ether/water mixture. After allowing the mixture to stir well for several minutes, the resulting biphasic solution was poured into a $250 \mathrm{~mL}$ separatory funnel. The aqueous layer was removed and the organic layer was washed with $20 \mathrm{~mL}$ of a saturated $\mathrm{NaHCO}_{3}$ aqueous solution and 20 $\mathrm{mL}$ of saturated $\mathrm{NH}_{4} \mathrm{Cl}$ aqueous solution. The organic layer was dried over $\mathrm{Na}_{2} \mathrm{SO}_{4}$, filtered, and the filtrate was concentrated under reduced pressure to give the desired diazo $\beta$-keto ester as a viscous yellow liquid that was taken on without further purification $(1.09 \mathrm{~g}, 92 \%)$. 


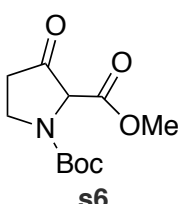

$\boldsymbol{\beta}$-Keto ester s6: ${ }^{13}$ To a solution of diazo $\beta$-keto ester s6c $(0.900 \mathrm{~g}, 3.31 \mathrm{mmol})$ in $33 \mathrm{~mL}$ of toluene was added $\mathrm{Rh}_{2}(\mathrm{OAc})_{4}$ $(0.007 \mathrm{~g}, 0.016 \mathrm{mmol})$. The resulting mixture was heated to $90{ }^{\circ} \mathrm{C}$ under Ar, and effervescence was observed. Once effervescence ceased (approximately $30 \mathrm{~min}$ ), the reaction mixture was allowed to cool to room temperature. The reaction mixture was concentrated in vacuo. The resulting green residue was diluted with diethyl ether and filtered through Celite. The filtrate was evaporated under reduced pressure to give the product, a yellow oil, as 64:36 mixture of rotamers $(0.775 \mathrm{~g}, 96 \%)$. This material is sufficiently pure to be converted to the vinyl triflate without any further purification. The spectral data matched that reported by Sorensen and co-workers: ${ }^{13}{ }^{1} \mathrm{H} \mathrm{NMR}\left(500 \mathrm{MHz}, \mathrm{CDCl}_{3}\right) \delta 4.45$ and $4.38(\mathrm{~s}, 1 \mathrm{H}), 3.77-3.68(\mathrm{~m}, 5 \mathrm{H}), 2.60(\mathrm{br} \mathrm{s}, 2 \mathrm{H}), 1.38$ and $1.32(\mathrm{~s}, 9 \mathrm{H})$. ATR-FTIR (thin film): 2977, 1775, 1746, $1707,1395,1239,1163 \mathrm{~cm}^{-1}$.

\section{B. Preparation of 3-Aryl-2-butanones.}

\section{General Procedure.}
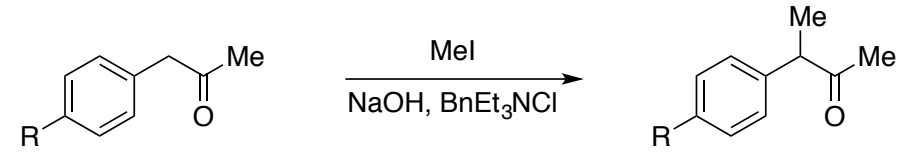

(s9)

A mixture of benzyl methyl ketone $(2.80 \mathrm{~g}, 20.9 \mathrm{mmol}), 50 \%$ aqueous solution of $\mathrm{NaOH}(10.4 \mathrm{~mL}, 129 \mathrm{mmol})$, and benzyltrimethylammonium chloride $(0.166 \mathrm{~g}, 0.73 \mathrm{mmol})$ was stirred vigorously using a mechanical stirrer. Methyl iodide $(1.94 \mathrm{~mL}, 31.3 \mathrm{mmol})$ was added slowly to this mixture, whose temperature of the solution was kept around room temperature using an ice bath. After $1 \mathrm{~h}, 20 \mathrm{~mL}$ of $\mathrm{H}_{2} \mathrm{O}$ and $30 \mathrm{~mL}$ of ethyl acetate were added to the reaction mixture. The organic layer was separated, and was washed with $\mathrm{H}_{2} \mathrm{O}$ until its $\mathrm{pH}$ became neutral. The resulting organic phase was washed with $10 \mathrm{~mL}$ of brine. The organic layer was separated and dried over $\mathrm{Na}_{2} \mathrm{SO}_{4}$, filtrated, and the filtrate was concentrated in vacuo. Purification via MPLC afforded the product.

\section{Characterization Data for 3-Aryl-2-butanones.}

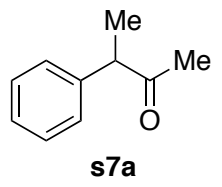

3-Phenyl-2-butanone s7a. ${ }^{15}$ The general procedure was followed using $2.80 \mathrm{~g}$ of benzyl methyl ketone (20.9 mmol), 10.4 $\mathrm{mL}$ of a $50 \%$ aqueous soln. of $\mathrm{NaOH}, 0.166 \mathrm{~g}$ of benzyltrimethylammonium chloride $(0.731 \mathrm{mmol})$ and $1.94 \mathrm{~mL}$ of MeI (31.3 mmol). Purification by MPLC (2:100 - 10:90 EtOAc: hexanes) afforded the product as a colorless liquid (1.96 g, $64 \%)$; the spectral data matched that reported by Maeda and co-workers: ${ }^{15}{ }^{1} \mathrm{H}$ NMR $\left(500 \mathrm{MHz}, \mathrm{CDCl}_{3}\right) \delta 7.31(\mathrm{t}, J=7.5$ $\mathrm{Hz}, 2 \mathrm{H}), 7.25(\mathrm{~m}, 1 \mathrm{H}), 7.20(\mathrm{~d}, J=8.0 \mathrm{~Hz}, 2 \mathrm{H}), 3.72(\mathrm{q}, J=7.0 \mathrm{~Hz}, 1 \mathrm{H}), 2.02(\mathrm{~s}, 3 \mathrm{H}), 1.37(\mathrm{~d}, J=7.0 \mathrm{~Hz}, 3 \mathrm{H})$. ATRFTIR (thin film): $3027,2976,2932,1711,1493,1452,1353,1164,1068 \mathrm{~cm}^{-1}$. 


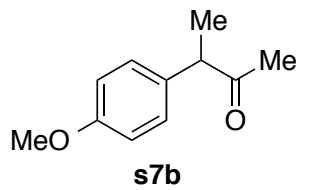

3-para-Methoxyphenyl-2-butanone $\mathbf{s 7 b} .^{16}$ The general procedure was followed using $1.33 \mathrm{~g}$ of benzyl methyl ketone $(8.09 \mathrm{mmol}), 4.00 \mathrm{~mL}$ of a $50 \%$ aqueous soln. of $\mathrm{NaOH}, 0.0640 \mathrm{~g}$ of benzyltrimethylammonium chloride $(0.283 \mathrm{mmol})$ and $0.750 \mathrm{~mL}$ of MeI $(12.1 \mathrm{mmol})$. Purification by MPLC (2:100 - 10:90 EtOAc: hexanes) afforded the product as a white solid $(0.576 \mathrm{~g} 50 \%)$; the spectral data matched that reported by Yamataka and co-workers: ${ }^{16}{ }^{1} \mathrm{H} \mathrm{NMR}(500 \mathrm{MHz}$, $\mathrm{CDCl}_{3}$,) $\delta 7.05(\mathrm{~d}, J=8.5 \mathrm{~Hz}, 2 \mathrm{H}), 6.79(\mathrm{~d}, J=8.5 \mathrm{~Hz}, 2 \mathrm{H}), 3.69(\mathrm{~s}, 3 \mathrm{H}), 3.61(\mathrm{q}, J=7.0 \mathrm{~Hz}, 1 \mathrm{H}), 1.95(\mathrm{~s}, 3 \mathrm{H}), 1.27(\mathrm{~d}, J$ $=7.0 \mathrm{~Hz}, 3 \mathrm{H})$.

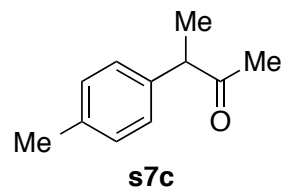

3-para-Methylphenyl-2-butanone s7c. ${ }^{16}$ The general procedure was followed using $1.02 \mathrm{~g}$ of 4-methylbenzyl methyl ketone $(6.88 \mathrm{mmol}), 3.30 \mathrm{~mL}$ of a $50 \%$ aqueous soln. of $\mathrm{NaOH}, 0.054 \mathrm{~g}$ of benzyltrimethylammonium chloride $(0.241$ $\mathrm{mmol})$ and $0.64 \mathrm{~mL}$ of MeI $(10.3 \mathrm{mmol})$. Purification by MPLC $(2: 100-10: 90$ EtOAc: hexanes $)$ afforded the product as a colorless oil $(0.903 \mathrm{~g}, 81 \%)$; the spectral data matched that reported by Yamataka and co-workers: ${ }^{16}{ }^{1} \mathrm{H}$ NMR (500 $\left.\mathrm{MHz}, \mathrm{CDCl}_{3}\right) \delta 7.14(\mathrm{~d}, J=8.0 \mathrm{~Hz}, 2 \mathrm{H}), 7.11(\mathrm{~d}, J=8.0 \mathrm{~Hz}, 2 \mathrm{H}), 3.69(\mathrm{q}, J=7.0 \mathrm{~Hz}, 1 \mathrm{H}), 2.32(\mathrm{~s}, 3 \mathrm{H}), 2.03(\mathrm{~s}, 3 \mathrm{H}), 1.36$ $\left(\mathrm{d}, J=7.0 \mathrm{~Hz}, 3 \mathrm{H}\right.$ ). ATR-FTIR (thin film) 2975, 2930, 1712, 1512, 1453, 1353, 1164, $1067 \mathrm{~cm}^{-1}$.

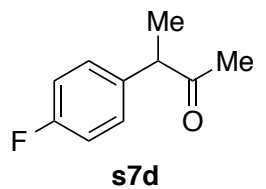

3-p-Fluorophenyl-2-butanone s7d. ${ }^{17}$ The general procedure was followed using $0.500 \mathrm{~g}$ of 4-fluorobenzyl methyl ketone (3.28 mmol), $1.62 \mathrm{~mL}$ of a $50 \%$ aqueous soln. of $\mathrm{NaOH}, 0.026 \mathrm{~g}$ of benzyltrimethylammonium chloride and $0.31 \mathrm{~mL}$ of MeI $(4.92 \mathrm{mmol})$. Purification by MPLC (2:100 - 10:90 EtOAc: hexanes) afforded the product as a colorless liquid $(0.374 \mathrm{~g}, 68 \%)$; the spectral data matched that reported by Fu and co-workers: ${ }^{17}{ }^{1} \mathrm{H} \mathrm{NMR}\left(500 \mathrm{MHz}, \mathrm{CDCl}_{3}\right) \delta 7.17(\mathrm{dd}, J$ $=9.5 \mathrm{~Hz}, 5.5 \mathrm{~Hz}, 2 \mathrm{H}), 7.02(\mathrm{t}, J=9.0 \mathrm{~Hz}, 2 \mathrm{H}), 3.73(\mathrm{q}, J=7.0 \mathrm{~Hz}, 1 \mathrm{H}), 2.05(\mathrm{~s}, 3 \mathrm{H}), 3.37(\mathrm{~d}, J=7.0 \mathrm{~Hz}, 3 \mathrm{H})$. ATRFTIR (thin film) 2978, 2933, 1712, 1600, 1507, 1354, 1221, 1158, $1067 \mathrm{~cm}^{-1}$.

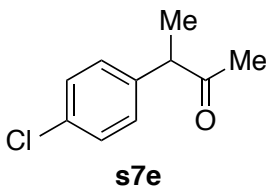

3-para-Chlorophenyl-2-butanone s7e. ${ }^{16}$ The general procedure was followed using $1.08 \mathrm{~g}$ of 4-chlorobenzyl methyl ketone $(6.40 \mathrm{mmol}), 3.30 \mathrm{~mL}$ of a $50 \%$ aqueous soln. of $\mathrm{NaOH}, 0.051 \mathrm{~g}$ of benzyltrimethylammonium chloride and 0.60 $\mathrm{mL}$ of MeI $(9.60 \mathrm{mmol})$. Purification by MPLC (2:100 - 10:90 EtOAc: hexanes) afforded the product as a colorless liquid $(0.895 \mathrm{~g}, 77 \%)$; the spectral data matched that reported by Yamataka and co-workers: ${ }^{16}{ }^{1} \mathrm{H} \mathrm{NMR}\left(500 \mathrm{MHz}, \mathrm{CDCl}_{3}\right)$ $\delta 7.22(\mathrm{~d}, J=8.5 \mathrm{~Hz}, 2 \mathrm{H}), 7.11(\mathrm{~d}, J=8.5 \mathrm{~Hz}, 2 \mathrm{H}), 3.69(\mathrm{q}, J=7.0 \mathrm{~Hz}, 1 \mathrm{H}), 2.00(\mathrm{~s}, 3 \mathrm{H}), 1.32(\mathrm{~d}, J=7.0 \mathrm{~Hz}, 3 \mathrm{H})$. ATRFTIR (thin film): 2977, 2933, 1711, 1489, 1408, 1353, 1163, 1091, $1014 \mathrm{~cm}^{-1}$. 


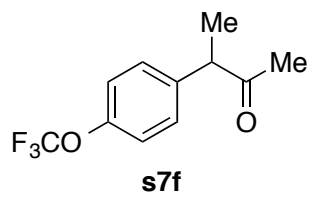

3-para-Trifluoromethoxyphenyl-2-butanone s7f. The general procedure was followed using $0.468 \mathrm{~g}$ of 4 trifluoromethoxybenzyl methyl ketone $(2.14 \mathrm{mmol}), 1.00 \mathrm{~mL}$ of a $50 \%$ aqueous soln. of $\mathrm{NaOH}$, and $0.017 \mathrm{~g}$ of benzyltrimethylammonium chloride $(0.075 \mathrm{mmol})$ and $0.20 \mathrm{~mL}$ of MeI $(3.21 \mathrm{mmol})$. Purification by MPLC (2:100 10:90 EtOAc: hexanes) afforded the product as a colorless liquid $(0.277 \mathrm{~g}, 56 \%):{ }^{1} \mathrm{H}$ NMR $\left(500 \mathrm{MHz}, \mathrm{CDCl}_{3}\right) \delta 7.25(\mathrm{~m}$, $2 \mathrm{H}), 7.18(\mathrm{~m}, 2 \mathrm{H}), 3.79(\mathrm{~m}, 7.0 \mathrm{~Hz}, 1 \mathrm{H}), 2.07(\mathrm{~s}, 3 \mathrm{H}), 1.38(\mathrm{~d}, J=7.0 \mathrm{~Hz}, 3 \mathrm{H}) ;{ }^{13} \mathrm{C} \mathrm{NMR}\left(125 \mathrm{MHz}, \mathrm{CDCl}_{3}\right) \delta 208.2(\mathrm{C})$, $148.3(\mathrm{C}), 139.2(\mathrm{C}), 129.2(\mathrm{CH}), 121.4(\mathrm{CH}), 120.4\left(\mathrm{q}, J_{C F}=255.1 \mathrm{~Hz}, \mathrm{CF}_{3}\right), 52.9(\mathrm{CH}), 28.5\left(\mathrm{CH}_{3}\right), 17.4\left(\mathrm{CH}_{3}\right)$. ATRFTIR (thin film): 2980, 1715, 1508, 1356, 1255, 1209, $1158 \mathrm{~cm}^{-1}$.

\section{Preparation of Cyclohexane-1,3-dione Derivatives.}

\section{Preparation of 2-Phenylcyclohexane-1,3-dione.}

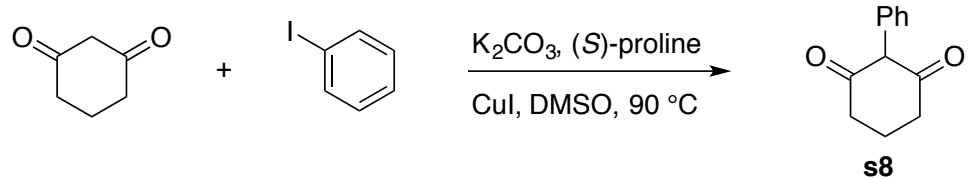

(s10)

2-Phenylcyclohexane-1,3-dione $\mathbf{s 8} .^{18}$ To a solution of $0.138 \mathrm{~g}$ of CuI $(0.725 \mathrm{mmol}), 0.167 \mathrm{~g}$ of L-proline $(1.45 \mathrm{mmol})$, $3.07 \mathrm{~g}$ of $\mathrm{K}_{2} \mathrm{CO}_{3}(29.0 \mathrm{mmol})$ and $2.43 \mathrm{~g}$ of diketone $(21.7 \mathrm{mmol})$ in $30 \mathrm{~mL}$ of DMSO was added $1.48 \mathrm{~g}$ of iodobenzene in $5 \mathrm{~mL}$ of DMSO. The reaction mixture was heated to $90{ }^{\circ} \mathrm{C}$. After $48 \mathrm{~h}$, the reaction mixture was cooled to room temperature, and the cooled mixture was poured into $15 \mathrm{~mL}$ of a $1 \mathrm{M}$ aqueous soln. of $\mathrm{HCl}$. The phases were separated, and the organic layer was extracted with EtOAc $(3 \times 20 \mathrm{~mL})$. The combined organic extracts were washed with $20 \mathrm{~mL}$ of brine and dried over $\mathrm{Na}_{2} \mathrm{SO}_{4}$, filtered and, the filtrate was concentrated in vacuo. Purification by MPLC (2:100 - 10:90 EtOAc: hexanes) afforded the product as a yellow solid $(1.32 \mathrm{~g}, 97 \%)$; the spectral data matched that reported by Wheeler: ${ }^{18}{ }^{1} \mathrm{H}$ NMR $\left(500 \mathrm{MHz}, \mathrm{CDCl}_{3}\right) \delta 7.37(\mathrm{t}, J=7.5 \mathrm{~Hz}, 2 \mathrm{H}), 7.28(\mathrm{~m}, 1 \mathrm{H}), 7.14(\mathrm{~d}, J=7.0 \mathrm{~Hz}, 2 \mathrm{H}), 2.44(\mathrm{t}, J=6.0$ $\mathrm{Hz}, 4 \mathrm{H}), 2.02-1.96$ (m, 2H). ATR-FTIR (thin film): $2825-3250,1704,1672,1595,1449,1240,1105 \mathrm{~cm}^{-1}$.

\section{Preparation of 4,6-Dimethylcyclohexane-1,3-Dione.}
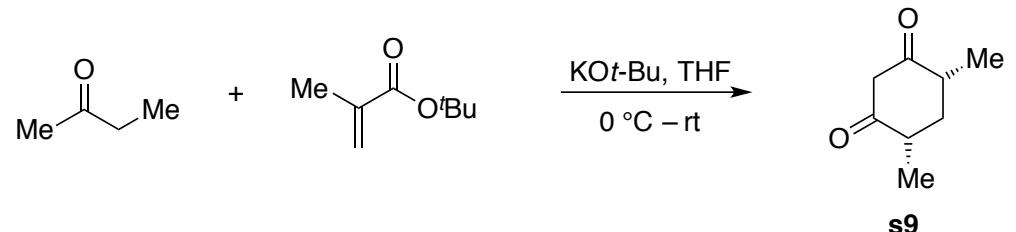

$(s 11)$

4,6-Dimethylcyclohexane-1,3-dione s9. ${ }^{19}$ To a solution of $0.66 \mathrm{~mL}$ of 2-butanone $(7.38 \mathrm{mmol})$ in $60 \mathrm{~mL}$ of THF was added $7.38 \mathrm{~mL}$ of $\mathrm{KO} t$-Bu in THF $(7.38 \mathrm{mmol})$ at $0{ }^{\circ} \mathrm{C}$. After $5 \mathrm{~min}, 1.00 \mathrm{~mL}$ of tert-butyl methacrylate $(6.15 \mathrm{mmol})$ was added dropwise. The reaction mixture was allowed to warm to room temperature. After 30 min, the reactives were quenched by the addition of $5 \mathrm{~mL}$ of a $1 \mathrm{M}$ aqueous soln. of $\mathrm{HCl}$. The resulting mixture was extracted with ethyl acetate $(3 \times 10 \mathrm{~mL})$. The combined organic layers were washed with $10 \mathrm{~mL}$ of a saturated aqueous solution of $\mathrm{NaHCO}_{3}$ solution, followed by $10 \mathrm{~mL}$ of brine. The resulting organic phase was dried over $\mathrm{Na}_{2} \mathrm{SO}_{4}$, filtered, and the filtrate was concentrated in vacuo. Purification via MPLC (3:97 to 10:90 EtOAc:hexanes) afforded the major cis-isomer as a white solid $(0.534 \mathrm{~g}, 62 \%)$. The spectral data matched that reported by Ishikawa, Satio and co-workers: ${ }^{19}{ }^{1} \mathrm{H} \mathrm{NMR}(500 \mathrm{MHz}$, $\left.\mathrm{CDCl}_{3}\right) \delta 3.45$ and $3.34(\mathrm{ABq}, J=16.0 \mathrm{~Hz}, 2 \mathrm{H}), 2.69-2.64(\mathrm{~m}, 2 \mathrm{H}), 2.12(\mathrm{dt}, J=13.5 \mathrm{~Hz}, 5.5 \mathrm{~Hz}, 1 \mathrm{H}), 1.12(\mathrm{~d}, J=6.5$ $\mathrm{Hz}, 6 \mathrm{H})$, only visible peaks; ${ }^{13} \mathrm{C}$ NMR $\left(125 \mathrm{MHz}, \mathrm{CDCl}_{3}\right)$ o $204.6(\mathrm{C}), 58.2\left(\mathrm{CH}_{2}\right), 44.7(\mathrm{CH}), 35.8\left(\mathrm{CH}_{2}\right), 13.9\left(\mathrm{CH}_{3}\right)$. 
ATR-FTIR (thin film): 2966, 2932, 2874, 1684, 1707, 1567, 1456, 1319, 1266, 1215, 1162, $1110 \mathrm{~cm}^{-1}$.

\section{Synthesis of Vinyl Triflates:}

\section{General Procedure:}

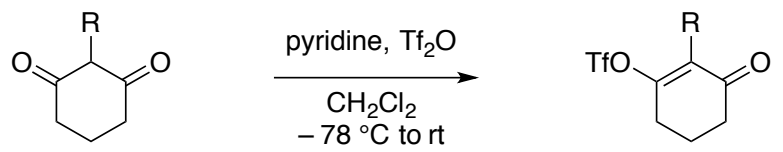

Method A: To a $-78{ }^{\circ} \mathrm{C}$ solution of cyclohexane-1,3-dione $(0.261 \mathrm{~g}, 2.33 \mathrm{mmol})$ and pyridine $(0.38 \mathrm{~mL}, 4.66 \mathrm{mmol})$ in dichloromethane $(10 \mathrm{~mL})$ was slowly added trifluoromethanesulfonic anhydride $(0.47 \mathrm{~mL}, 2.80 \mathrm{mmol})$. After $10 \mathrm{~min}$, the reaction mixture warmed to room temperature. After 4 hours, the reactives were quenched through the addition of $5 \mathrm{~mL}$ of a $1 \mathrm{M}$ aqueous soln. of $\mathrm{HCl}$. The resulting mixture was extracted with diethyl ether $(3 \times 10 \mathrm{~mL})$. The combined organic layers were washed with $10 \mathrm{~mL}$ of a saturated aqueous solution of $\mathrm{NaHCO}_{3}$ solution, followed by $10 \mathrm{~mL}$ of brine. The resulting organic phase was dried over $\mathrm{Na}_{2} \mathrm{SO}_{4}$, filtered, and the filtrate was concentrated in vacuo. Purification via MPLC (3:97 to 10:90 EtOAc:hexanes) afforded the product.

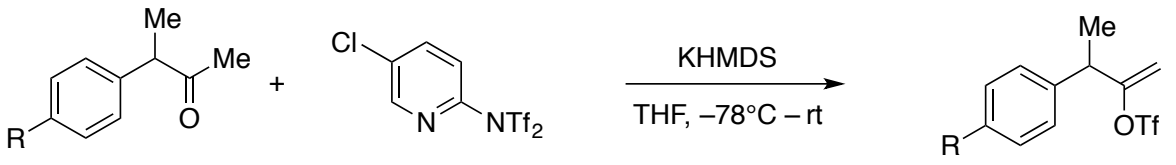

Method B: To a $-78{ }^{\circ} \mathrm{C}$ solution of KHMDS (1.1 equiv) in THF (0.2 M) was slowly added a solution of 3-aryl-2butanone (1.0 equiv) in THF. After $30 \mathrm{~min}$, a solution of Comins' reagent (1.1 equiv) in THF was added dropwise. The resulting mixture was then gradually warmed to room temperature. After stirring for $15 \mathrm{~h}$, the reactives were quenched by the addition of a saturated aqueous solution of $\mathrm{NH}_{4} \mathrm{Cl}$. The resulting mixture was extracted with EtOAc. The combined organic phases were washed by brine, and the resulting organic phases were dried over $\mathrm{Na}_{2} \mathrm{SO}_{4}$, filtered and the filtrate was concentrated in vacuo. Purification by MPLC (2:98 to 5:95 EtOAc:hexanes) afforded the product.

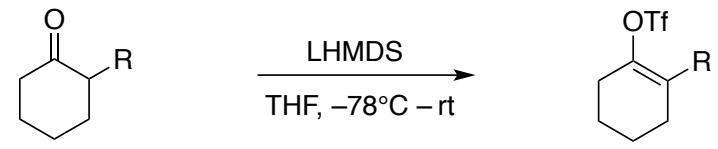

Method C: To a $-78{ }^{\circ} \mathrm{C}$ solution of LHMDS (1.2 equiv) in THF $(0.2 \mathrm{M})$ was slowly added a solution of 2methylcyclohexanone (1.0 equiv) in THF. After stirring for $0.5 \mathrm{~h}$, a solution of $\operatorname{PhNTf}_{2}(1.2$ equiv) in THF was added dropwise to the reaction. The resulting mixture was then gradually warmed to room temperature. After stirring for $15 \mathrm{~h}$, the reactives were quenched by the addition of a saturated aqueous solution of $\mathrm{NH}_{4} \mathrm{Cl}$. The mixture was then extracted with EtOAc. The combined organic phases were washed by brine, and the resulting organic phases were dried over $\mathrm{Na}_{2} \mathrm{SO}_{4}$, filtered and the filtrate was concentrated in vacuo. Purification by MPLC (2:98 to 5:95 EtOAc:hexanes) afforded the product.
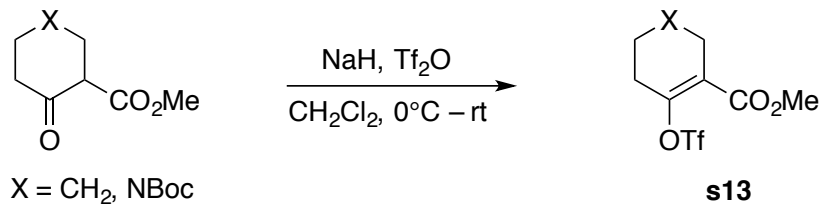

(s15)

Method D: To a $0{ }^{\circ} \mathrm{C}$ solution of $\beta$-ketoester (1.0 equiv) in $\mathrm{CH}_{2} \mathrm{Cl}_{2}(0.2 \mathrm{M})$ was slowly added $\mathrm{NaH}(60 \%$ dispersed in mineral oil, 1.2 equiv). After $30 \mathrm{~min}$, trifluoromethanesulfonic anhydride (1.2 equiv) was added dropwise. The resulting mixture was then warmed to room temperature. After stirring for $15 \mathrm{~h}$, the reactives were quenched by the addition of 
water. The mixture was then extracted with $\mathrm{CH}_{2} \mathrm{Cl}_{2}$. The combined organic phases were washed by brine, and the resulting organic phases were dried over $\mathrm{Na}_{2} \mathrm{SO}_{4}$, filtered and the filtrate was concentrated in vacuo. Purification by MPLC (2:98 to 5:95 EtOAc:hexanes) afforded the product.

\section{Characterization Data for Vinyl Triflates.}

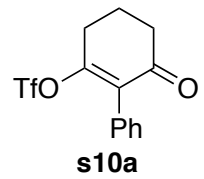

Vinyl triflate s10a. Method A was followed using $1.61 \mathrm{~g}$ of 2-phenylcyclohexane-1,3-dione $\mathbf{s 8}(8.55 \mathrm{mmol}), 1.39 \mathrm{~mL}$ of pyridine $(17.1 \mathrm{mmol})$ and $1.73 \mathrm{~mL}$ of $\mathrm{Tf}_{2} \mathrm{O}(10.2 \mathrm{mmol})$ in $42 \mathrm{~mL}$ of $\mathrm{CH}_{2} \mathrm{Cl}_{2}$. Purification by MPLC (2:98 to 10:90 EtOAc:hexanes) afforded the product as a colorless oil $(1.83 \mathrm{~g}, 61 \%)$ : ${ }^{1} \mathrm{H}$ NMR $\left(500 \mathrm{MHz}, \mathrm{CDCl}_{3}\right) \delta 7.44-7.37(\mathrm{~m}, 3 \mathrm{H})$, $7.17(\mathrm{~d}, J=7.5 \mathrm{~Hz}, 2 \mathrm{H}), 2.90(\mathrm{t}, J=6.5 \mathrm{~Hz}, 2 \mathrm{H}), 2.62(\mathrm{t}, J=7.0 \mathrm{~Hz}, 2 \mathrm{H}), 2.19(\mathrm{q}, J=6.5 \mathrm{~Hz}, 2 \mathrm{H}) ;{ }^{13} \mathrm{C} \mathrm{NMR}(125 \mathrm{MHz}$, $\left.\mathrm{CDCl}_{3}\right) \delta 196.5(\mathrm{C}), 162.0(\mathrm{C}), 132.7(\mathrm{C}), 130.0(\mathrm{CH}), 129.1(\mathrm{C}), 128.8(\mathrm{CH}), 128.1(\mathrm{CH}), 117.9\left(\mathrm{q}, J_{C F}=317.5 \mathrm{~Hz}, \mathrm{CF}_{3}\right)$, $37.2\left(\mathrm{CH}_{2}\right), 29.2\left(\mathrm{CH}_{2}\right), 20.5\left(\mathrm{CH}_{2}\right)$.

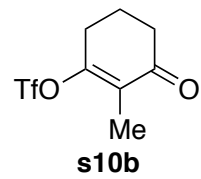

Vinyl triflate $\mathbf{s 1 0 b} .^{20}$ Method A was followed using $1.26 \mathrm{~g}$ of 2-methylcyclohexane-1,3-dione (10.0 mmol), $1.60 \mathrm{~mL}$ of pyridine $(20.0 \mathrm{mmol})$ and $2.00 \mathrm{~mL}$ of $\mathrm{Tf}_{2} \mathrm{O}(1.20 \mathrm{mmol})$ in $100 \mathrm{~mL}$ of $\mathrm{CH}_{2} \mathrm{Cl}_{2}$. Purification by MPL (2:98 to 10:90 EtOAc:hexanes) afforded the product as a colorless oil $(2.39 \mathrm{~g}, 93 \%)$. Vinyl triflate s10b was previously reported by Fürstner and co-workers: ${ }^{20}{ }^{1} \mathrm{H}$ NMR $\left(500 \mathrm{MHz}, \mathrm{CDCl}_{3}\right) \delta 2.75-2.73(\mathrm{~m}, 2 \mathrm{H}), 2.49(\mathrm{t}, J=7.0 \mathrm{~Hz}, 2 \mathrm{H}), 2.09$ (quint, 6.5 $\mathrm{Hz}, 2 \mathrm{H}), 1.86$ (t, $J=2.0 \mathrm{~Hz}, 3 \mathrm{H}$ ); ATR-FTIR (thin film): 2929, 1688, 1419, 1264, 1242, $1138 \mathrm{~cm}^{-1}$.

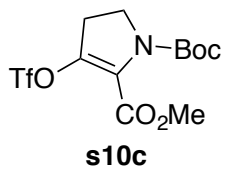

Vinyl triflate s10c. ${ }^{21}$ Method B was followed using $0.358 \mathrm{~g}$ of $\beta$-ketoester s6 $(1.47 \mathrm{mmol}), 0.694 \mathrm{~g}$ of Comins' reagent $(1.77 \mathrm{mmol})$ and $0.440 \mathrm{~g}$ of KHMDS $(2.20 \mathrm{mmol})$ in $11 \mathrm{~mL}$ of THF. Purification by MPLC (2:98 to 10:90 EtOAc:hexanes) afforded the product as a colorless oil $(0.352 \mathrm{~g}, 64 \%)$; vinyl triflate s10c was previously reported by Cativiela and co-workers: ${ }^{21}{ }^{1} \mathrm{H}$ NMR $\left(500 \mathrm{MHz}, \mathrm{CDCl}_{3}\right) \delta 3.92(\mathrm{t}, J=9.5 \mathrm{~Hz}, 2 \mathrm{H}), 3.80(\mathrm{~s}, 3 \mathrm{H}), 2.90(\mathrm{t}, J=9.5 \mathrm{~Hz}, 2 \mathrm{H})$, 1.38 (s, 9H); ATR-FTIR (thin film): 2981, 1750, 1716, 1429, 1407, 1370, 1324, 1216, 1139, 1030, $989 \mathrm{~cm}^{-1}$.

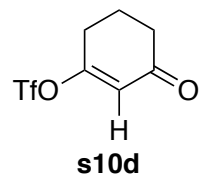

Vinyl triflate s10d. ${ }^{22}$ Method A was followed using $0.261 \mathrm{~g}$ of cyclohexane-1,3-dione $(2.33 \mathrm{mmol}), 0.38 \mathrm{~mL}$ of pyridine (4.66 mmol) and $0.47 \mathrm{~mL}$ of $\mathrm{Tf}_{2} \mathrm{O}(2.80 \mathrm{mmol})$ in $23 \mathrm{~mL}$ of $\mathrm{CH}_{2} \mathrm{Cl}_{2}$. Purification by MPLC (2:98 to 10:90 EtOAc:hexanes) afforded the product as a colorless oil $(0.305 \mathrm{~g}, 54 \%)$; vinyl triflate s10d was previously reported by Sieber and co-workers: ${ }^{22}{ }^{1} \mathrm{H}$ NMR $\left(500 \mathrm{MHz}, \mathrm{CDCl}_{3}\right) \delta 6.02(\mathrm{~s}, 1 \mathrm{H}), 2.66(\mathrm{dt}, J=6.5 \mathrm{~Hz}, 1 \mathrm{~Hz}, 2 \mathrm{H}), 2.42(\mathrm{t}, J=6.5 \mathrm{~Hz}$, $2 \mathrm{H}), 2.10$ (quint, $J=6.5 \mathrm{~Hz}, 2 \mathrm{H}) ;{ }^{13} \mathrm{C} \mathrm{NMR}\left(125 \mathrm{MHz}, \mathrm{CDCl}_{3}\right) \delta 197.3(\mathrm{C}), 167.4(\mathrm{C}), 119.2(\mathrm{CH}), 118.4\left(\mathrm{q}, J_{C F}=317.5\right.$ $\left.\mathrm{Hz}, \mathrm{CF}_{3}\right), 36.3\left(\mathrm{CH}_{2}\right), 28.4\left(\mathrm{CH}_{2}\right), 20.7\left(\mathrm{CH}_{2}\right)$. ATR-FTIR (thin film): 2965, 1689. 1645, 1421, 1245, 1205, 1133,1068 $\mathrm{cm}^{-1}$ 


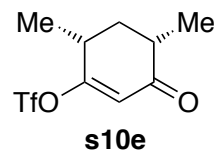

Vinyl triflate s10e. Method A was followed using $0.198 \mathrm{~g}$ of 4,6-dimethylcyclohexane-1,3-dione s9 (1.41 mmol), 0.23 $\mathrm{mL}$ of pyridine $(2.82 \mathrm{mmol})$ and $0.29 \mathrm{~mL}$ of $\mathrm{Tf}_{2} \mathrm{O}(1.69 \mathrm{mmol})$ in $14 \mathrm{~mL}$ of $\mathrm{CH}_{2} \mathrm{Cl}_{2}$. Purification by MPLC (2:98 to $10: 90$ EtOAc:hexanes) afforded the product as a colorless oil $(0.367 \mathrm{~g}, 96 \%) .{ }^{1} \mathrm{H}$ NMR $\left(500 \mathrm{MHz}, \mathrm{CDCl}_{3}\right) \delta 6.00(\mathrm{~s}, 1 \mathrm{H}), 2.94$ $(\mathrm{m}, 1 \mathrm{H}), 2.40(\mathrm{~m}, 1 \mathrm{H}), 2.17(\mathrm{dt}, J=13.5 \mathrm{~Hz}, 5.0 \mathrm{~Hz}, 1 \mathrm{H}), 1.47(\mathrm{dt}, J=13.5 \mathrm{~Hz}, 11.0 \mathrm{~Hz}, 1 \mathrm{H}), 1.23(\mathrm{~d}, J=7.0 \mathrm{~Hz}, 3 \mathrm{H})$, $1.10(\mathrm{~d}, J=6.5 \mathrm{~Hz}, 3 \mathrm{H}) ;{ }^{13} \mathrm{C} \mathrm{NMR}\left(125 \mathrm{MHz}, \mathrm{CDCl}_{3}\right) \delta 199.3(\mathrm{C}), 169.4(\mathrm{C}), 118.4\left(\mathrm{q}, J_{C F}=318.7 \mathrm{~Hz}, \mathrm{CF}_{3}\right), 118.3(\mathrm{CH})$, $41.1(\mathrm{CH}), 38.3(\mathrm{CH}), 34.3\left(\mathrm{CH}_{2}\right), 17.2\left(\mathrm{CH}_{3}\right), 14.0\left(\mathrm{CH}_{3}\right)$. ATR-FTIR (thin film): 2917, 2849, 1694, 1425, 1246, 1215, $1139 \mathrm{~cm}^{-1}$.

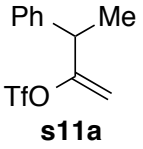

Vinyl triflate s11a. Method B was followed using $1.33 \mathrm{~g}$ of KHMDS (6.66 mmol), $0.898 \mathrm{~g}$ of ketone s7a $(6.06 \mathrm{mmol})$ and $2.61 \mathrm{~g}$ of Comins' reagent $(6.66 \mathrm{mmol})$ in $30 \mathrm{~mL}$ of THF. Purification by MPLC (2:98 to 10:90 EtOAc:hexanes) afforded the product as a yellow oil $(0.958 \mathrm{~g}, 56 \%)$ : ${ }^{1} \mathrm{H}$ NMR $\left(500 \mathrm{MHz}, \mathrm{CDCl}_{3}\right) \delta 7.40-7.37(\mathrm{~m}, 2 \mathrm{H}), 7.33-7.28(\mathrm{~m}$, $3 \mathrm{H}), 5.25(\mathrm{~d}, J=4.0 \mathrm{~Hz}, 1 \mathrm{H}), 5.05(\mathrm{dd}, J=4.0 \mathrm{~Hz}, 1.5 \mathrm{~Hz}, 1 \mathrm{H}), 3.76(\mathrm{q}, J=7.0 \mathrm{~Hz}, 1 \mathrm{H}), 1.52(\mathrm{~d}, J=7.0 \mathrm{~Hz}, 3 \mathrm{H}) ;{ }^{13} \mathrm{C}$ NMR $\left(125 \mathrm{MHz}, \mathrm{CDCl}_{3}\right) \delta 159.8(\mathrm{C}), 140.3(\mathrm{C}), 128.8(\mathrm{CH}), 127.5(\mathrm{CH}), 118.6\left(\mathrm{q}, J_{C F}=317 \mathrm{~Hz}, \mathrm{CF}_{3}\right), 103.9\left(\mathrm{CH}_{2}\right), 44.2$ $(\mathrm{CH}), 19.3\left(\mathrm{CH}_{3}\right)$, only visible peaks. ATR-FTIR (thin film): 2980, 1663, 1495, 1415, 1248, 1203, 1132, $1059 \mathrm{~cm}^{-1}$.

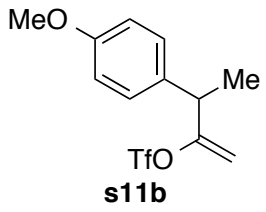

Vinyl triflate s11b. Method B was followed using $0.228 \mathrm{~g}$ of KHMDS (1.44 mmol), $0.234 \mathrm{~g}$ of ketone s7b (1.31 mmol) and $0.567 \mathrm{~g}$ of Comins' reagent $(1.44 \mathrm{mmol})$ in $7 \mathrm{~mL}$ of THF. Purification by MPLC (2:98 to 10:90 EtOAc:hexanes) afforded the product as a colorless oil $(0.244 \mathrm{~g}, 60 \%)$ : ${ }^{1} \mathrm{H}$ NMR $\left(500 \mathrm{MHz}, \mathrm{CDCl}_{3}\right) \delta 7.20(\mathrm{~d}, J=8.5 \mathrm{~Hz}, 2 \mathrm{H}), 6.91(\mathrm{~d}, J$ $=8.5 \mathrm{~Hz}, 2 \mathrm{H}), 5.21(\mathrm{~d}, J=4.0 \mathrm{~Hz}, 1 \mathrm{H}), 5.01(\mathrm{~d}, J=4.0 \mathrm{~Hz}, 1 \mathrm{H}), 3.81(\mathrm{~s}, 3 \mathrm{H}), 3.72(\mathrm{q}, J=7.0 \mathrm{~Hz}, 1 \mathrm{H}), 1.49(\mathrm{~d}, J=7.0$ $\mathrm{Hz}, 3 \mathrm{H}) ;{ }^{13} \mathrm{C}$ NMR $\left(125 \mathrm{MHz}, \mathrm{CDCl}_{3}\right) \delta 160.3(\mathrm{C}), 159.0(\mathrm{C}), 132.3(\mathrm{C}), 128.5(\mathrm{CH}), 118.5\left(\mathrm{q}, J_{C F}=317.6 \mathrm{~Hz}, \mathrm{CF}_{3}\right)$, $114.2(\mathrm{CH}), 103.6\left(\mathrm{CH}_{2}\right), 55.1\left(\mathrm{CH}_{3}\right), 43.5(\mathrm{CH}), 19.3\left(\mathrm{CH}_{3}\right)$. ATR-FTIR (thin film): 2978, 2838, 1662, 1611, 1542, $1414,1247,1203,1132,1066 \mathrm{~cm}^{-1}$.

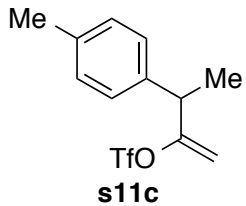

Vinyl triflate s11c. Method B was followed using $0.666 \mathrm{~g}$ of KHMDS (3.34 mmol), $0.492 \mathrm{~g}$ of ketone s7c $(3.03 \mathrm{mmol})$ and $1.31 \mathrm{~g}$ of Comins' reagent $(3.34 \mathrm{mmol})$ in $30 \mathrm{~mL}$ of THF. Purification by MPLC (2:98 to 10:90 EtOAc:hexanes) afforded the product as a colorless oil with $90 \%$ purity $(0.676 \mathrm{~g}, 76 \%)$. The crude vinyl triflate was used in the subsequent cross-coupling reaction without additional purification. 


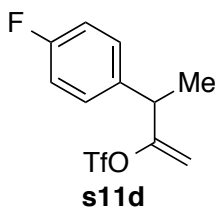

Vinyl triflate s11d. Method B was followed using $0.144 \mathrm{~g}$ of KHMDS $(0.72 \mathrm{mmol}), 0.100 \mathrm{~g}$ of ketone s7d $(0.60 \mathrm{mmol})$ and $0.259 \mathrm{~g}$ of Comins' reagent $(0.66 \mathrm{mmol})$ in $10 \mathrm{~mL}$ of THF. Purification by MPLC (2:98 to 10:90 EtOAc:hexanes) afforded the product as a colorless oil with $90 \%$ purity $(0.100 \mathrm{~g}, 56 \%)$. The crude vinyl triflate was used in the subsequent cross-coupling reaction without additional purification.

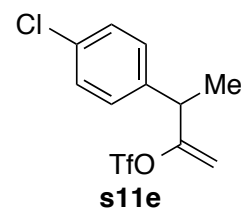

Vinyl triflate s11e. Method B was followed using $0.650 \mathrm{~g}$ of KHMDS $(3.26 \mathrm{mmol}), 0.541 \mathrm{~g}$ of ketone s7e $(2.96 \mathrm{mmol})$ and $1.28 \mathrm{~g}$ of Comins' reagent $(3.26 \mathrm{mmol})$ in $30 \mathrm{~mL}$ of THF. Purification by MPLC (2:98 to 10:90 EtOAc:hexanes) afforded the product as a colorless oil with $90 \%$ purity $(0.652 \mathrm{~g}, 70 \%)$. The crude vinyl triflate was used in the subsequent cross-coupling reaction without additional purification.

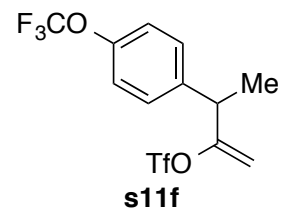

Vinyl triflate s11f. Method B was followed using $0.261 \mathrm{~g}$ of KHMDS (1.30 mmol), $0.253 \mathrm{~g}$ of ketone s7f $(1.08 \mathrm{mmol})$ and $0.467 \mathrm{~g}$ of Comins' reagent $(1.18 \mathrm{mmol})$ in $13 \mathrm{~mL}$ of THF. Purification by MPLC (2:98 to 10:90 EtOAc:hexanes) afforded the product as a colorless oil with $90 \%$ purity $(0.240 \mathrm{~g}, 60 \%)$. The crude vinyl triflate was used in the subsequent cross-coupling reaction without additional purification.

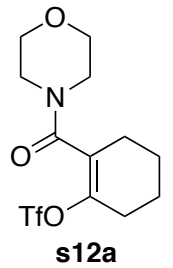

Vinyl triflate s12a. Method $\mathrm{C}$ was followed using $0.336 \mathrm{~g}$ of LHMDS (2.00 mmol), $0.387 \mathrm{~g}$ of $\beta$-ketoamide $\mathbf{s 5}$ (1.83 $\mathrm{mmol}$ ) and $0.720 \mathrm{~g}$ of $\mathrm{PhNTf}_{2}$ in $10 \mathrm{~mL}$ of THF. Purification by MPLC (2:98 to 10:90 EtOAc:hexanes) afforded the product as a colorless oil $(0.556 \mathrm{~g}, 88 \%)$ : ${ }^{1} \mathrm{H}$ NMR $\left(500 \mathrm{MHz}, \mathrm{CDCl}_{3}\right) \delta 3.54-3.29(\mathrm{~m}, 8 \mathrm{H}), 2.41-2.01(\mathrm{~m}, 4 \mathrm{H}), 1.68$ (br s, 2H), 1.56 (m, 2H); ATR-FTIR (thin film) 2933, 2861, 1626, 1435, 1410, 1299, 1245, 1204, $1114 \mathrm{~cm}^{-1}$.

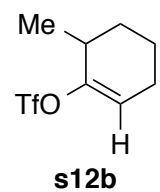

Vinyl triflate s12b. ${ }^{23}$ Method C was followed using $1.35 \mathrm{~g}$ of LHMDS (8.06 mmol), $0.826 \mathrm{~g}$ of 2-methylcyclohexanone $(7.36 \mathrm{mmol})$ and $2.89 \mathrm{~g}$ of $\mathrm{PhNTf}_{2}(8.10 \mathrm{mmol})$ in $80 \mathrm{~mL}$ of THF. Purification by MPLC (2:98 to 10:90 EtOAc: hexane) afforded the product as a colorless oil $(1.24 \mathrm{~g}, 69 \%)$; vinyl triflate $\mathbf{s 1 2 b}$ was previously reported by Cho and co-workers: ${ }^{23}$ ${ }^{1} \mathrm{H}$ NMR $\left(500 \mathrm{MHz}, \mathrm{CDCl}_{3}\right) \delta 5.72(\mathrm{dt}, J=4.0 \mathrm{~Hz}, 1.0 \mathrm{~Hz}, 1 \mathrm{H}), 2.56-2.51(\mathrm{~m}, 1 \mathrm{H}), 2.18-2.15(\mathrm{~m}, 2 \mathrm{H}), 1.96-1.90(\mathrm{~m}$, 
$1 \mathrm{H}), 1.67-1.62(\mathrm{~m}, 1 \mathrm{H}), 1.59-1.54(\mathrm{~m}, 1 \mathrm{H}), 1.49-1.43(\mathrm{~m}, 1 \mathrm{H}), 1.13(\mathrm{~d}, J=7.0 \mathrm{~Hz}, 3 \mathrm{H})$; ATR-FTIR (thin film): 2955, $2923,2853,1598,1494,1459,1378,1210,1144 \mathrm{~cm}^{-1}$.

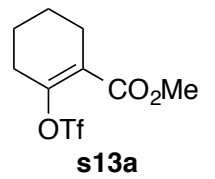

Triflate s13a. ${ }^{24}$ Method D was followed using $2.00 \mathrm{~mL}$ of methyl 2-oxocyclohexanecarboxylate (12.7 mmol), $0.608 \mathrm{~g}$ of $\mathrm{NaH}(15.2 \mathrm{mmol})$ and $2.56 \mathrm{~mL}$ of $\mathrm{Tf}_{2} \mathrm{O}(15.2 \mathrm{mmol})$ in $60 \mathrm{~mL}$ of $\mathrm{CH}_{2} \mathrm{Cl}_{2}$. The crude product was afforded as brown oil (3.65 g, 100\%). The spectral data matched that reported by Bols and co-workers: ${ }^{24}{ }^{1} \mathrm{H}$ NMR $\left(500 \mathrm{MHz}, \mathrm{CDCl}_{3}\right) \delta 3.78(\mathrm{~s}$, $3 \mathrm{H}), 2.46(\mathrm{dt}, J=5.6 \mathrm{~Hz}, 3.0 \mathrm{~Hz}, 2 \mathrm{H}), 2.38(\mathrm{dt}, J=5.6 \mathrm{~Hz}, 3.0 \mathrm{~Hz}, 2 \mathrm{H}), 1.77(\mathrm{dt}, J=5.8 \mathrm{~Hz}, 2.9 \mathrm{~Hz}, 2 \mathrm{H}), 1.65(\mathrm{dt}, J=5.8$ $\mathrm{Hz}, 2.9 \mathrm{~Hz}, 2 \mathrm{H}) ;{ }^{13} \mathrm{C}$ NMR $\left(125 \mathrm{MHz}, \mathrm{CDCl}_{3}\right) \delta 165.1(\mathrm{C}), 151.8(\mathrm{C}), 122.8(\mathrm{C}), 118.3\left(\mathrm{q}, J_{C F}=319.7 \mathrm{~Hz}, \mathrm{C}\right), 52.1\left(\mathrm{CH}_{3}\right)$, $28.6\left(\mathrm{CH}_{2}\right), 26.1\left(\mathrm{CH}_{2}\right), 22.2\left(\mathrm{CH}_{2}\right), 21.0\left(\mathrm{CH}_{2}\right)$. The crude vinyl triflate was used in the subsequent cross-coupling reaction without additional purification.

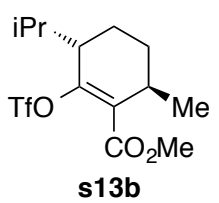

Vinyl triflate s13b. Method D was followed using $0.495 \mathrm{~g}$ of $\beta$-ketoester s4a ( $2.33 \mathrm{mmol}, 1.0$ equiv), $0.112 \mathrm{~g}$ of $\mathrm{NaH}$ (60\% dispersed in mineral oil, $2.79 \mathrm{mmol})$ and $0.47 \mathrm{~mL}$ of trifluoromethanesulfonic anhydride $(2.80 \mathrm{mmol}, 1.2$ equiv) in $10 \mathrm{~mL}$ of $\mathrm{CH}_{2} \mathrm{Cl}_{2}$. The crude product was afforded as brown oil $(3.65 \mathrm{~g}, 100 \%)$. Purification by MPLC (2:98 to 5:95 EtOAc:hexanes) afforded the product with 90:10 mixture of diastereomers as a brown liquid $(0.426 \mathrm{~g}, 53 \%)$ : ${ }^{1} \mathrm{H} \mathrm{NMR}$ $\left(500 \mathrm{MHz}, \mathrm{CDCl}_{3}\right) \delta 3.77(\mathrm{~s}, 3 \mathrm{H}), 2.99(\mathrm{~m}, 1 \mathrm{H}), 2.46(\mathrm{~m}, 1 \mathrm{H}), 2.20(\mathrm{~m}, 1 \mathrm{H}), 1.68-1.54(\mathrm{~m}, 4 \mathrm{H}), 1.03(\mathrm{~d}, J=7.0 \mathrm{~Hz}$, $3 \mathrm{H}), 0.98(\mathrm{~d}, J=7.0 \mathrm{~Hz}, 3 \mathrm{H}), 0.83(\mathrm{~d}, J=6.5 \mathrm{~Hz}, 3 \mathrm{H}) ;{ }^{13} \mathrm{C} \mathrm{NMR}\left(125 \mathrm{MHz}, \mathrm{CDCl}_{3}\right) \delta 165.5(\mathrm{C}), 153.6(\mathrm{C}), 129.7(\mathrm{C})$, $118.3\left(\mathrm{q}, J_{C F}=317.5 \mathrm{~Hz}, \mathrm{CF}_{3}\right), 52.1\left(\mathrm{CH}_{3}\right), 44.2(\mathrm{CH}), 31.4(\mathrm{CH}), 27.7(\mathrm{CH}), 27.3\left(\mathrm{CH}_{2}\right), 20.2\left(\mathrm{CH}_{3}\right), 19.0\left(\mathrm{CH}_{3}\right), 18.5$ $\left(\mathrm{CH}_{2}\right), 16.4\left(\mathrm{CH}_{3}\right)$; characteristic data for minor diastereomer: ${ }^{1} \mathrm{H}$ NMR $\left(500 \mathrm{MHz}, \mathrm{CDCl}_{3}\right) \delta 2.73(\mathrm{~m}, 1 \mathrm{H}), 2.40(\mathrm{~m}, 1 \mathrm{H})$, $1.81(\mathrm{~m}, 2 \mathrm{H}), 1.07(\mathrm{~d}, J=7.0 \mathrm{~Hz}, 3 \mathrm{H}) ;{ }^{13} \mathrm{C} \mathrm{NMR}\left(125 \mathrm{MHz}, \mathrm{CDCl}_{3}\right) \delta 51.9\left(\mathrm{CH}_{3}\right), 42.6(\mathrm{CH}), 31.8(\mathrm{CH}), 17.1\left(\mathrm{CH}_{3}\right)$. ATR-FTIR (thin film): 2958, 2927, 2873, 1728, 1421, 1311, 1207, $1141 \mathrm{~cm}^{-1}$

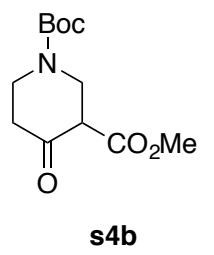

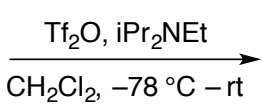

s4b

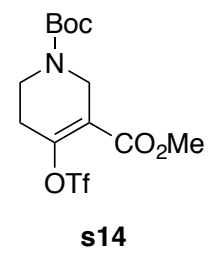

(s16)

Vinyl triflate s14..$^{25}$ To a $-78{ }^{\circ} \mathrm{C}$ solution of $\beta$-ketoester s4b $(0.649 \mathrm{~g}, 2.52 \mathrm{mmol})$ in $\mathrm{CH}_{2} \mathrm{Cl}_{2}(10 \mathrm{~mL})$ was slowly added $\operatorname{iPr}_{2} \mathrm{NEt}(2.2 \mathrm{~mL}, 12.6 \mathrm{mmol})$. After stirring for $20 \mathrm{~min}$, trifluoromethanesulfonic anhydride $(0.51 \mathrm{~mL}, 3.0 \mathrm{mmol}) \mathrm{was}$ added dropwise to the reaction mixture. The resulting mixture was then warmed to rt. After $2 \mathrm{~h}$, the reactives were quenched by the addition of $20 \mathrm{~mL}$ of water. The mixture was then extracted with $\mathrm{CH}_{2} \mathrm{Cl}_{2}$. The combined organic phases were washed by brine, dried over $\mathrm{Na}_{2} \mathrm{SO}_{4}$, filtered and the filtrate was concentrated in vacuo. Purification by MPLC (2:98 to 5:95 EtOAc:hexanes) afforded the product as yellow oil $(0.750 \mathrm{~g}, 76 \%)$. Vinyl triflate $\mathbf{s 1 4}$ was previously reported by Di Fabio and co-workers: ${ }^{25}{ }^{1} \mathrm{H}$ NMR $\left(500 \mathrm{MHz}, \mathrm{CDCl}_{3}\right) \delta 4.16(\mathrm{~s}, 2 \mathrm{H}), 3.71(\mathrm{~s}, 3 \mathrm{H}), 3.52(\mathrm{t}, J=5.5 \mathrm{~Hz}, 2 \mathrm{H}), 2.41(\mathrm{~s}, 2 \mathrm{H})$, $1.37(\mathrm{~s}, 9 \mathrm{H}) ;{ }^{13} \mathrm{C}$ NMR $\left(125 \mathrm{MHz}, \mathrm{CDCl}_{3}\right) \delta 170.8(\mathrm{C}), 162.6(\mathrm{C}), 153.8(\mathrm{C}), 120.5(\mathrm{C}), 118.2\left(\mathrm{q}, J_{C F}=319.5 \mathrm{~Hz}, \mathrm{C}\right), 80.7$ (C), 60.1 $\left(\mathrm{CH}_{2}\right), 52.1\left(\mathrm{CH}_{3}\right), 43.0\left(\mathrm{CH}_{2}\right), 28.8\left(\mathrm{CH}_{2}\right), 28.1\left(\mathrm{CH}_{3}\right)$. 


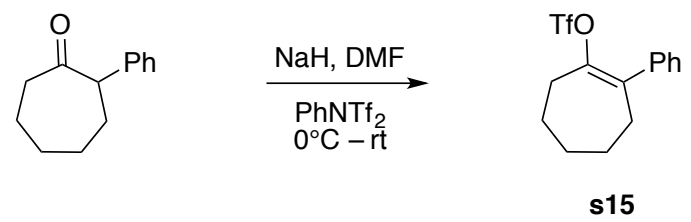

Vinyl triflate s15. To a flame-dried round-bottom flask, $0.368 \mathrm{~g}$ of 2-phenylcycloheptanone (1.95 mmol) was added to a suspension of $0.034 \mathrm{~g}$ of $\mathrm{NaH}(60 \%$ dispersed in mineral oil, $2.34 \mathrm{mmol})$ in $10 \mathrm{~mL}$ of DMF at $0{ }^{\circ} \mathrm{C}$. The mixture was warmed to room temperature. After 30 minutes, $0.835 \mathrm{~g}$ of 1,1,1-trifluoro- $N$-phenyl- $N$-(trifluoromethylsulfonyl)methanesulfonamide $(2.34 \mathrm{mmol})$ was added. After an additional 12 hours, the reaction mixture was diluted with $20 \mathrm{~mL}$ of water and $50 \mathrm{~mL}$ of ethyl acetate. The phases were separated, and the organic phase was washed with $20 \mathrm{~mL}$ of brine and $20 \mathrm{~mL}$ of water. The resulting organic phase was dried over $\mathrm{Na}_{2} \mathrm{SO}_{4}$, filtered, and the filtrate was concentrated in vacuo. The crude mixture was purified by $\operatorname{MPLC}(3: 97-20: 80$ EtOAc:hexanes) to afford the product as a transparent oil (0.375g, 60\%): ${ }^{1} \mathrm{H}$ NMR (500 MHz, $\left.\mathrm{CDCl}_{3}\right) \delta 7.36(\mathrm{t}, J=7.5 \mathrm{~Hz}, 2 \mathrm{H}), 7.30(\mathrm{~m}, 1 \mathrm{H}), 7.25(\mathrm{~m}, 2 \mathrm{H}), 2.70(\mathrm{~m}, 2 \mathrm{H}), 2.56$ $(\mathrm{m}, 2 \mathrm{H}), 1.84-1.77(\mathrm{~m}, 6 \mathrm{H}) ;{ }^{13} \mathrm{C}$ NMR $\left(125 \mathrm{MHz}, \mathrm{CDCl}_{3}\right) \delta 147.5(\mathrm{C}), 139.1(\mathrm{C}), 131.1(\mathrm{C}), 128.2(\mathrm{CH}), 127.9(\mathrm{CH})$, $127.6(\mathrm{CH}), 118.1\left(\mathrm{q}, J_{C F}=317.5 \mathrm{~Hz}, \mathrm{CF}_{3}\right), 34.0\left(\mathrm{CH}_{2}\right), 35.4\left(\mathrm{CH}_{2}\right), 30.9\left(\mathrm{CH}_{2}\right), 25.9\left(\mathrm{CH}_{2}\right), 24.5\left(\mathrm{CH}_{2}\right)$. ATR-FTIR (thin film): 2923, 2853, 1706, 1445, 1413, 1244, 1205, 1140, $990 \mathrm{~cm}^{-1}$

\section{Synthesis of 2-Alkenylaryl Amines by Suzuki Cross-Coupling Reaction.}

\section{A. General procedure.}
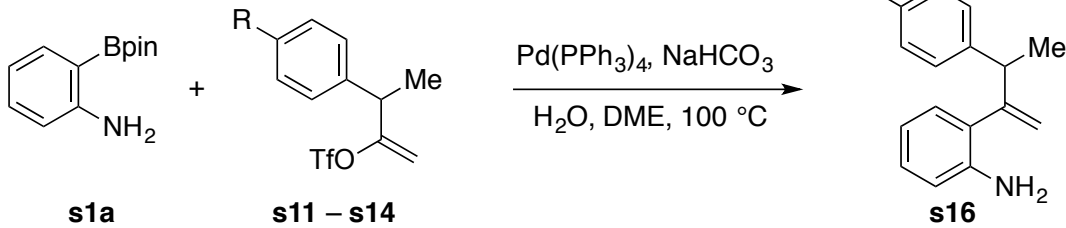

(s18)

To a mixture of vinyl triflate $\mathbf{s 1 1}$ - $\mathbf{s 1 4}$ (1 equiv), 2-aminoarylboronic acid pinacol ester s1a (1.1 equiv), and $\operatorname{Pd}\left(\mathrm{PPh}_{3}\right)_{4}(5$ mol \%) in dimethoxyethane $(0.1 \mathrm{M})$ was added a saturated aqueous solution of $\mathrm{NaHCO}_{3}(2 \mathrm{~mL} / \mathrm{mmol}$ of boronic ester). The resulting mixture was heated to $100{ }^{\circ} \mathrm{C}$. After $4 \mathrm{~h}$, the mixture was cooled to room temperature and diluted with 5 $\mathrm{mL}$ of cold water. The solution was extracted with $2 \times 10 \mathrm{~mL}$ of ether followed by $10 \mathrm{~mL}$ of brine. The resulting organic phase was dried over $\mathrm{Na}_{2} \mathrm{SO}_{4}$ and was concentrated in vacuo. Purification by MPLC (3:97 to 50:50 EtOAc:hexanes) afforded the product $\mathbf{s 1 6 .}$

\section{B. Synthesis of 2-Alkenylaryl Amines.}

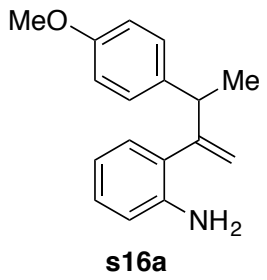

Styryl aniline s16a. The general procedure was followed by using $0.362 \mathrm{~g}$ of 2-aminoarylboronic acid pinacol ester s1a $(1.65 \mathrm{mmol}), 0.466 \mathrm{~g}$ of vinyl triflate $\mathbf{~ s 1 1 b}(1.50 \mathrm{mmol}), 0.173 \mathrm{~g}$ of $\mathrm{Pd}\left(\mathrm{PPh}_{3}\right)_{4}(5 \mathrm{~mol} \%), 3.0 \mathrm{~mL}$ of a saturated aq. soln. of $\mathrm{NaHCO}_{3}$ and $15 \mathrm{~mL}$ of dimethoxyethane. Purification by MPLC (3:97 to 10:90 EtOAc:hexanes) afforded the product as yellow oil $(0.270 \mathrm{~g}, 71 \%)$ : ${ }^{1} \mathrm{H}$ NMR $\left(500 \mathrm{MHz}, \mathrm{CDCl}_{3}\right) \delta 7.17(\mathrm{~d}, J=8.5 \mathrm{~Hz}, 2 \mathrm{H}), 7.06(\mathrm{dt}, J=8.0 \mathrm{~Hz}, 1.5 \mathrm{~Hz}, 1 \mathrm{H}), 6.85$ $(\mathrm{m}, 3 \mathrm{H}), 6.67(\mathrm{~m}, 2 \mathrm{H}), 5.33(\mathrm{~s}, 1 \mathrm{H}), 5.18(\mathrm{~s}, 1 \mathrm{H}), 3.81(\mathrm{q}, J=7.0 \mathrm{~Hz}, 1 \mathrm{H}), 3.80(\mathrm{~s}, 3 \mathrm{H}), 3.67(\mathrm{br} \mathrm{s}, 2 \mathrm{H}), 1.50(\mathrm{~d}, J=7.5$ 
$\mathrm{Hz}, 3 \mathrm{H}) ;{ }^{13} \mathrm{C}$ NMR $\left(125 \mathrm{MHz}, \mathrm{CDCl}_{3}\right) \delta 158.1(\mathrm{C}), 152.3(\mathrm{C}), 143.5(\mathrm{C}), 136.4(\mathrm{C}), 129.1(\mathrm{CH}), 128.8(\mathrm{C}), 128.7(\mathrm{CH})$, $127.8(\mathrm{CH}), 117.8(\mathrm{CH}), 115.4(\mathrm{CH}), 114.2\left(\mathrm{CH}_{2}\right), 113.7(\mathrm{CH}), 55.2\left(\mathrm{CH}_{3}\right), 45.4(\mathrm{CH}), 20.3\left(\mathrm{CH}_{3}\right)$. ATR-FTIR (thin film): $3459,3373,2962,2930,2833,1609,1582,1508,1492,1449,1242,1176,1032 \mathrm{~cm}^{-1}$.

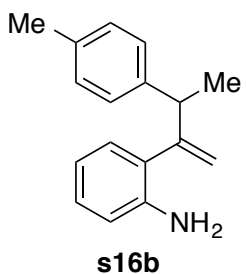

Styryl aniline s16b. The general procedure was followed by using $0.405 \mathrm{~g}$ of 2-aminoarylboronic acid pinacol ester s1a $(1.85 \mathrm{mmol}), 0.494 \mathrm{~g}$ of vinyl triflate s11c $(1.68 \mathrm{mmol}), 0.194 \mathrm{~g}$ of $\mathrm{Pd}\left(\mathrm{PPh}_{3}\right)_{4}(5 \mathrm{~mol} \%), 3.6 \mathrm{~mL}$ of a saturated aq. soln. of $\mathrm{NaHCO}_{3}$ and $20 \mathrm{~mL}$ of dimethoxyethane. Purification by MPLC (3:97 to 10:90 EtOAc:hexanes) afforded the product as yellow oil $(0.320 \mathrm{~g}, 80 \%):{ }^{1} \mathrm{H}$ NMR $\left(500 \mathrm{MHz}, \mathrm{CDCl}_{3}\right) \delta 7.18-7.13(\mathrm{~m}, 4 \mathrm{H}), 7.07(\mathrm{dt}, J=8.0 \mathrm{~Hz}, 1.5 \mathrm{~Hz}, 1 \mathrm{H}), 6.86(\mathrm{dd}$, $J=7.5 \mathrm{~Hz}, 1.0 \mathrm{~Hz}, 1 \mathrm{H}), 6.72-6.67(\mathrm{~m}, 2 \mathrm{H}), 5.35(\mathrm{~s}, 1 \mathrm{H}), 5.19(\mathrm{~s}, 1 \mathrm{H}), 3.83(\mathrm{q}, 1 \mathrm{H}), 3.68(\mathrm{~s}, 2 \mathrm{H}), 2.38(\mathrm{~s}, 3 \mathrm{H}), 1.52(\mathrm{~d}$, $3 \mathrm{H}) ;{ }^{13} \mathrm{C}$ NMR $\left(125 \mathrm{MHz}, \mathrm{CDCl}_{3}\right) \delta 152.1(\mathrm{C}), 143.4(\mathrm{C}), 141.3(\mathrm{C}), 135.8(\mathrm{C}), 129.1(\mathrm{CH}), 129.0(\mathrm{CH}), 128.2(\mathrm{C}), 127.8$ $(\mathrm{CH}), 127.7(\mathrm{CH}), 117.9(\mathrm{CH}), 115.4(\mathrm{CH}), 114.4\left(\mathrm{CH}_{2}\right), 45.7(\mathrm{CH}), 21.1\left(\mathrm{CH}_{3}\right), 20.3\left(\mathrm{CH}_{3}\right)$. ATR-FTIR (thin film): $3460,3373,3019,2966,2926,1610,1511,1492,1449,1295,1259,1065 \mathrm{~cm}^{-1}$.

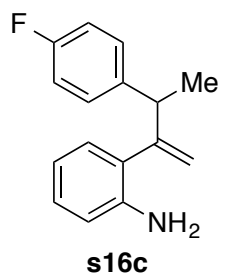

Styryl aniline s16c. The general procedure was followed by using $0.166 \mathrm{~g}$ of 2-aminoarylboronic acid pinacol ester s1a $(0.76 \mathrm{mmol}), 0.200 \mathrm{~g}$ of vinyl triflate s11d $(0.69 \mathrm{mmol}), 0.040 \mathrm{~g}$ of $\mathrm{Pd}\left(\mathrm{PPh}_{3}\right)_{4}(5 \mathrm{~mol} \%), 1.5 \mathrm{~mL}$ of a saturated aq. soln. of $\mathrm{NaHCO}_{3}$ and $10 \mathrm{~mL}$ of dimethoxyethane. Purification by MPLC (3:97 to 10:90 EtOAc:hexanes) afforded the product as yellow oil $(0.122 \mathrm{~g}, 73 \%)$ : ${ }^{1} \mathrm{H}$ NMR $\left(500 \mathrm{MHz}, \mathrm{CDCl}_{3}\right) \delta 7.17-7.14(\mathrm{~m}, 2 \mathrm{H}), 7.03(\mathrm{dt}, J=8.0 \mathrm{~Hz}, 1.5 \mathrm{~Hz}, 1 \mathrm{H}), 6.97-$ $6.93(\mathrm{~m}, 2 \mathrm{H}), 6.76(\mathrm{dd}, J=8.0 \mathrm{~Hz}, 1.5 \mathrm{~Hz}, 1 \mathrm{H}), 6.66-6.63(\mathrm{~m}, 2 \mathrm{H}), 5.29(\mathrm{~s}, 1 \mathrm{H}), 5.14(\mathrm{~s}, 1 \mathrm{H}), 3.80(\mathrm{q}, J=7.0 \mathrm{~Hz}, 1 \mathrm{H})$, $3.62(\mathrm{br} \mathrm{s}, 2 \mathrm{H}), 2.92(\mathrm{~d}, J=7.0 \mathrm{~Hz}, 3 \mathrm{H}) ;{ }^{13} \mathrm{C}$ NMR $\left(125 \mathrm{MHz}, \mathrm{CDCl}_{3}\right) \delta 161.5\left(\mathrm{~d}, J_{C F}=242.3 \mathrm{~Hz}, \mathrm{C}\right), 151.8(\mathrm{C}), 143.3$ $(\mathrm{C}), 139.9(\mathrm{C}), 129.1\left(\mathrm{~d}, J_{C F}=7.37 \mathrm{~Hz}, \mathrm{CH}\right), 129.0(\mathrm{CH}), 128.5(\mathrm{C}), 127.9(\mathrm{CH}), 117.9(\mathrm{CH}), 115.4(\mathrm{CH}), 115.0(\mathrm{~d}, J=$ $20.1 \mathrm{~Hz}, \mathrm{CH}), 114.5\left(\mathrm{CH}_{2}\right), 45.3(\mathrm{CH}), 20.2\left(\mathrm{CH}_{3}\right) ;{ }^{19} \mathrm{~F}$ NMR $\left(282 \mathrm{MHz}, \mathrm{CDCl}_{3}\right) \delta-117.5$. ATR-FTIR (thin film): 3469 , $3380,3023,2966,1612,1506,1493,1450,1218,1157 \mathrm{~cm}^{-1}$.

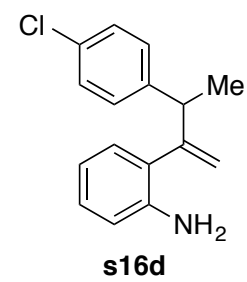

Styryl aniline s16d. The general procedure was followed by using $0.385 \mathrm{~g}$ of 2-aminoarylboronic acid pinacol ester s1a $(1.76 \mathrm{mmol}), 0.503 \mathrm{~g}$ of vinyl triflate s11e $(1.60 \mathrm{mmol}), 0.184 \mathrm{~g}$ of $\mathrm{Pd}\left(\mathrm{PPh}_{3}\right)_{4}(10 \mathrm{~mol} \%), 3.2 \mathrm{~mL}$ of a saturated aq. soln. of $\mathrm{NaHCO}_{3}$ and $15 \mathrm{~mL}$ of dimethoxyethane. Purification by MPLC (3:97 to 10:90 EtOAc:hexanes) afforded the product with $85 \%$ purity as yellow oil $(0.333 \mathrm{~g}, 81 \%)$. The aniline s15d compound was carried on to the azidation step without further purification. 


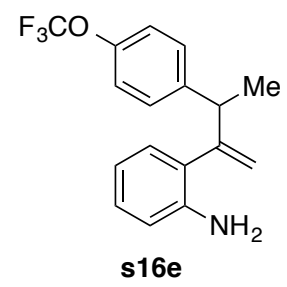

Styryl aniline s16e. The general procedure was followed by using $0.139 \mathrm{~g}$ of 2-aminoarylboronic acid pinacol ester s1a $(0.63 \mathrm{mmol}), 0.210 \mathrm{~g}$ of vinyl triflate $\mathbf{s 1 1 f}(0.58 \mathrm{mmol}), 0.033 \mathrm{~g}$ of $\mathrm{Pd}\left(\mathrm{PPh}_{3}\right)_{4}(5 \mathrm{~mol} \%), 1.2 \mathrm{~mL}$ of a saturated aq. soln. of $\mathrm{NaHCO}_{3}$ and $10 \mathrm{~mL}$ of dimethoxyethane. Purification by MPLC (3:97 to 10:90 EtOAc:hexanes) afforded the product as yellow oil $(0.180 \mathrm{~g}, 75 \%)$ : ${ }^{1} \mathrm{H}$ NMR $\left(500 \mathrm{MHz}, \mathrm{CDCl}_{3}\right) \delta 7.22(\mathrm{~d}, J=8.5 \mathrm{~Hz}, 2 \mathrm{H}), 7.10(\mathrm{~d}, J=8.5 \mathrm{~Hz}, 2 \mathrm{H}), 7.02(\mathrm{t}, J=$ $7.5 \mathrm{~Hz}, 1 \mathrm{H}), 6.73(7.0 \mathrm{~Hz}, 1 \mathrm{H}), 6.36(\mathrm{~m}, 2 \mathrm{H}), 5.28(\mathrm{~s}, 1 \mathrm{H}), 5.14(\mathrm{~s}, 1 \mathrm{H}), 3.83(\mathrm{q}, J=7.0 \mathrm{~Hz}, 1 \mathrm{H}), 3.60(\mathrm{~s}, 2 \mathrm{H}), 1.45(\mathrm{~d}, J=$ $7.0 \mathrm{~Hz}, 3 \mathrm{H}) ;{ }^{13} \mathrm{C}$ NMR $\left(125 \mathrm{MHz}, \mathrm{CDCl}_{3}\right) \delta 151.3(\mathrm{C}), 147.6(\mathrm{C}), 143.2(\mathrm{C}), 142.9(\mathrm{C}), 129.0(\mathrm{CH}), 128.9(\mathrm{CH}), 128.3$ $(\mathrm{C}), 127.9(\mathrm{CH}), 120.1(\mathrm{CH}), 117.9(\mathrm{CH}), 115.4(\mathrm{CH}), 114.8\left(\mathrm{CH}_{2}\right), 45.3(\mathrm{CH}), 20.1\left(\mathrm{CH}_{3}\right)$, only peaks visible; ${ }^{19} \mathrm{~F} \mathrm{NMR}$ $\left(282 \mathrm{MHz}, \mathrm{CDCl}_{3}\right) \delta$-58.3. ATR-FTIR (thin film): 3469, 3380, 2971, 1612, 1506, 1493, 1450, 1253, 1219, 1156, 1066, $1018 \mathrm{~cm}^{-1}$.

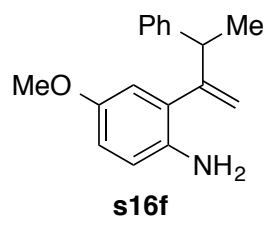

Styryl aniline s16f. The general procedure was followed by using $0.146 \mathrm{~g}$ of 2 -aminoarylboronic acid pinacol ester s1b $(0.59 \mathrm{mmol}), 0.149 \mathrm{~g}$ of vinyl triflate s11a $(0.53 \mathrm{mmol}), 0.031 \mathrm{~g}$ of $\mathrm{Pd}\left(\mathrm{PPh}_{3}\right)_{4}(5 \mathrm{~mol} \%), 1.2 \mathrm{~mL}$ of saturated aq. soln. of $\mathrm{NaHCO}_{3}$ and $10 \mathrm{~mL}$ of dimethoxyethane. Purification by MPLC (3:97 to 10:90 EtOAc: hexane) afforded the product as yellow oil $(0.091 \mathrm{~g}, 68 \%)$ : ${ }^{1} \mathrm{H}$ NMR $\left(500 \mathrm{MHz} \mathrm{CDCl}_{3}\right) \delta 7.30-7.21(\mathrm{~m}, 5 \mathrm{H}), 6.64(\mathrm{dd}, J=8.5 \mathrm{~Hz}, 3.0 \mathrm{~Hz}, 1 \mathrm{H}), 6.59(\mathrm{~d}$, $J=8.5 \mathrm{~Hz}, 1 \mathrm{H}), 6.36(\mathrm{~d}, J=3.0 \mathrm{~Hz}, 1 \mathrm{H}), 5.33(\mathrm{~s}, 1 \mathrm{H}), 5.15(\mathrm{~s}, 1 \mathrm{H}), 3.84(\mathrm{q}, J=7.0 \mathrm{~Hz}, 1 \mathrm{H}), 3.64(\mathrm{~s}, 3 \mathrm{H}), 3.37(\mathrm{~s}, 2 \mathrm{H})$, $1.50(\mathrm{~d}, J=7.0 \mathrm{~Hz}, 3 \mathrm{H}) ;{ }^{13} \mathrm{C}$ NMR $\left(125 \mathrm{MHz}, \mathrm{CDCl}_{3}\right) \delta 152.0(\mathrm{C}), 151.8(\mathrm{C}), 144.2(\mathrm{C}), 136.9(\mathrm{C}), 129.9(\mathrm{C}), 128.3$ $(\mathrm{CH}), 128.0(\mathrm{CH}), 126.4(\mathrm{CH}), 116.6(\mathrm{CH}), 114.6(\mathrm{CH}), 114.6\left(\mathrm{CH}_{2}\right), 113.8(\mathrm{CH}), 55.6\left(\mathrm{CH}_{3}\right), 46.0(\mathrm{CH}), 20.1\left(\mathrm{CH}_{3}\right)$; ATR-FTIR (thin film): 3538, 3358, 3025, 2965, 2829, 1599, 1496, 1451, 1423, 1278, 1209, $1039 \mathrm{~cm}^{-1}$.

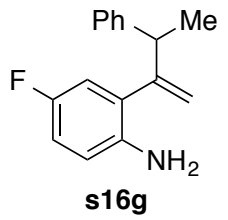

Styryl aniline s16g. The general procedure was followed by using $0.179 \mathrm{~g}$ of 2-aminoarylboronic acid pinacol ester s1d $(0.75 \mathrm{mmol}), 0.192 \mathrm{~g}$ of vinyl triflate s11a $(0.68 \mathrm{mmol}), 0.040 \mathrm{~g}$ of $\mathrm{Pd}\left(\mathrm{PPh}_{3}\right)_{4}(5 \mathrm{~mol} \%), 1.3 \mathrm{~mL}$ of a saturated aq. soln. of $\mathrm{NaHCO}_{3}$ and $10 \mathrm{~mL}$ of dimethoxyethane. Purification by MPLC (3:97 to 10:90 EtOAc:hexanes) afforded the product as yellow oil $(0.135 \mathrm{~g}, 82 \%)$ : ${ }^{1} \mathrm{H}$ NMR $\left(500 \mathrm{MHz}, \mathrm{CDCl}_{3}\right) \delta 7.31-7.28(\mathrm{~m}, 2 \mathrm{H}), 7.34-7.22(\mathrm{~m}, 3 \mathrm{H}), 6.76(\mathrm{dt}, J=8.0 \mathrm{~Hz}$, $3.0 \mathrm{~Hz}, 1 \mathrm{H}), 6.56(\mathrm{~m}, 2 \mathrm{H}), 5.36(\mathrm{~s}, 1 \mathrm{H}), 5.17(\mathrm{~s}, 1 \mathrm{H}), 3.80(\mathrm{q}, J=7.0 \mathrm{~Hz}, 1 \mathrm{H}), 3.48(\mathrm{~s}, 2 \mathrm{H}), 1.51(\mathrm{~d}, J=7.0 \mathrm{~Hz}, 3 \mathrm{H}) ;{ }^{13} \mathrm{C}$ NMR (125 MHz, $\left.\mathrm{CDCl}_{3}\right)$ d. $155.8\left(\mathrm{~d}, J_{C F}=233.2 \mathrm{~Hz}, \mathrm{C}\right), 151.1(\mathrm{C}), 143.8(\mathrm{C}), 139.5(\mathrm{C}), 129.9\left(\mathrm{~d}, J_{C F}=7.5 \mathrm{~Hz}, \mathrm{C}\right)$, $128.4(\mathrm{CH}), 127.8(\mathrm{CH}), 126.5(\mathrm{CH}), 116.2\left(\mathrm{~d}, J_{C F}=7.37 \mathrm{~Hz}, \mathrm{CH}\right), 115.4\left(\mathrm{~d}, J_{C F}=22.7 \mathrm{~Hz}, \mathrm{CH}\right), 115.1\left(\mathrm{CH}_{2}\right), 114.2(\mathrm{~d}$, $\left.J_{C F}=22.0 \mathrm{~Hz}, \mathrm{CH}\right), 45.9(\mathrm{CH}), 20.1\left(\mathrm{CH}_{3}\right)$; ATR-FTIR (thin film): 3439, 3373, 3026, 2968, 1601, 1584, 1494, 1264, 1180 $\mathrm{cm}^{-1}$. 


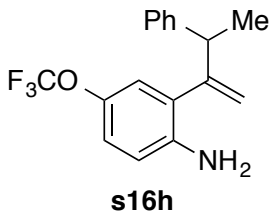

Styryl aniline s16. The general procedure was followed by using $0.262 \mathrm{~g}$ of 2-aminoarylboronic acid pinacol ester s1f $(0.86 \mathrm{mmol}), 0.218 \mathrm{~g}$ of vinyl triflate s11a $(0.79 \mathrm{mmol}), 0.046 \mathrm{~g}$ of $\mathrm{Pd}\left(\mathrm{PPh}_{3}\right)_{4}(5 \mathrm{~mol} \%), 1.6 \mathrm{~mL}$ of a saturated aq. soln. of $\mathrm{NaHCO}_{3}$ and $10 \mathrm{~mL}$ of dimethoxyethane. Purification by MPLC (3:97 to 10:90 EtOAc:hexanes) afforded the product as yellow oil $(0.180 \mathrm{~g}, 75 \%)$ : ${ }^{1} \mathrm{H}$ NMR $\left(500 \mathrm{MHz} \mathrm{CDCl}_{3}\right) \delta 7.30-7.27(\mathrm{~m}, 2 \mathrm{H}), 7.23-7.20(\mathrm{~m}, 3 \mathrm{H}), 6.89(\mathrm{dd}, J=8.5 \mathrm{~Hz}$, $2.5 \mathrm{~Hz}, 1 \mathrm{H}), 6.65(\mathrm{~d}, J=2.5 \mathrm{~Hz}, 1 \mathrm{H}), 6.58(\mathrm{~d}, J=9.0 \mathrm{~Hz}, 1 \mathrm{H}), 5.40(\mathrm{~s}, 1 \mathrm{H}), 5.18(\mathrm{~s}, 1 \mathrm{H}), 3.79(\mathrm{q}, J=7.0 \mathrm{~Hz}, 1 \mathrm{H}), 3.64(\mathrm{~s}$, $2 \mathrm{H}), 1.50(\mathrm{~d}, J=7.0 \mathrm{~Hz}, 3 \mathrm{H}) ;{ }^{13} \mathrm{C}$ NMR $\left(125 \mathrm{MHz}, \mathrm{CDCl}_{3}\right) \delta 150.7(\mathrm{C}), 143.7(\mathrm{C}), 142.3(\mathrm{C}), 140.6(\mathrm{C}), 129.2(\mathrm{C}), 128.4$ $(\mathrm{CH}), 127.7(\mathrm{CH}), 126.5(\mathrm{CH}), 122.2(\mathrm{CH}), 120.8(\mathrm{CH}), 120.7\left(\mathrm{q}, J_{C F}=253.5 \mathrm{~Hz}, \mathrm{CF}_{3}\right), 115.6(\mathrm{CH}), 115.1\left(\mathrm{CH}_{2}\right), 46.0$ $(\mathrm{CH}), 19.9\left(\mathrm{CH}_{3}\right)$. ATR-FTIR (thin film): 3442, 3368, 3027, 2970, 1618, 1601, 1495, 1244, $1215 \mathrm{~cm}^{-1}$.

\section{Synthesis of 2-Alkenylaryl Azides.}

\section{A. Preparation of 2-Alkenylaryl Azides by Suzuki Cross-Coupling Reaction.}

\section{General Procedure:}
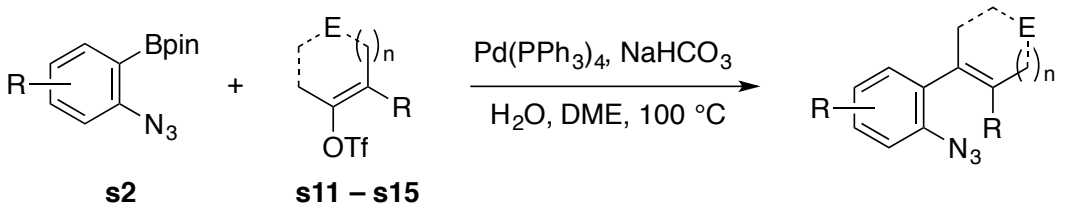

To a mixture of vinyl triflate s11 - s15 (1 equiv), 2-azidoarylboronic acid pinacol ester s2 (1.1 equiv), and $\operatorname{Pd}\left(\mathrm{PPh}_{3}\right)_{4}(10$ mol \%) in dimethoxyethane $(0.1 \mathrm{M})$ was added a saturated aqueous solution of $\mathrm{NaHCO}_{3}(2 \mathrm{~mL} / \mathrm{mmol}$ of boronic ester). The resulting mixture was heated to $100{ }^{\circ} \mathrm{C}$. After $1 \mathrm{~h}$, the mixture was cooled to room temperature and diluted with 5 $\mathrm{mL}$ of cold water. The solution was extracted with $2 \times 10 \mathrm{~mL}$ of ether followed by $10 \mathrm{~mL}$ of brine. The resulting organic phase was dried over $\mathrm{Na}_{2} \mathrm{SO}_{4}$, filtered and the filtrate was concentrated in vacuo. Purification by MPLC (3:97 to 10:90 EtOAc:hexanes) afforded the product.

\section{Preparation of 2-Alkenylaryl Azides}

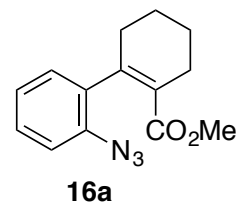

Styryl azide 16a. ${ }^{26}$ The general procedure was followed by using $0.107 \mathrm{~g}$ of 2-azidophenyl boronate s2a $(0.440 \mathrm{mmol})$, $0.114 \mathrm{~g}$ of vinyl triflate s13a $(0.400 \mathrm{mmol}), 0.0462 \mathrm{~g}$ of $\mathrm{Pd}\left(\mathrm{PPh}_{3}\right)_{4}(0.0400 \mathrm{mmol}), 0.8 \mathrm{~mL}$ of a saturated aq. soln. of $\mathrm{NaHCO}_{3}$ and $4 \mathrm{~mL}$ of dimethoxyethane. Purification by MPLC (3:97 to 10:90 EtOAc:hexanes) afforded the product as a yellow oil $(0.070 \mathrm{~g}, 70 \%)$. The spectral data matched that reported by Driver and co-workers: ${ }^{26}{ }^{1} \mathrm{H} \mathrm{NMR}(500 \mathrm{MHz}$, $\left.\mathrm{CDCl}_{3}\right) \delta 7.28(\mathrm{t}, J=7.7 \mathrm{~Hz}, 1 \mathrm{H}), 7.13(\mathrm{~d}, J=8.0 \mathrm{~Hz}, 1 \mathrm{H}), 7.09(\mathrm{t}, J=7.5 \mathrm{~Hz}, 1 \mathrm{H}), 6.99(\mathrm{dd}, J=7.5 \mathrm{~Hz}, 1.2 \mathrm{~Hz}, 1 \mathrm{H})$, $3.44(\mathrm{~s}, 3 \mathrm{H}), 2.45(\mathrm{~s}, 3 \mathrm{H}), 2.24(\mathrm{dt}, J=24.5 \mathrm{~Hz}, 5.9 \mathrm{~Hz}, 1 \mathrm{H}), 1.74(\mathrm{~d}, J=3.7 \mathrm{~Hz}, 4 \mathrm{H}) ;{ }^{13} \mathrm{C} \mathrm{NMR}\left(125 \mathrm{MHz}, \mathrm{CDCl}_{3}\right) \delta$ $168.4(\mathrm{C}), 145.3(\mathrm{C}), 136.3(\mathrm{C}), 135.8(\mathrm{C}), 128.5(\mathrm{CH}), 128.3(\mathrm{C}), 128.1(\mathrm{CH}), 124.7(\mathrm{CH}), 118.3(\mathrm{CH}), 51.2\left(\mathrm{CH}_{3}\right), 32.9$ $\left(\mathrm{CH}_{2}\right)$, $26.1\left(\mathrm{CH}_{2}\right), 22.1\left(\mathrm{CH}_{2}\right), 22.0\left(\mathrm{CH}_{2}\right)$; ATR-FTIR (thin film): 2939, 2127, 1720, $1248 \mathrm{~cm}^{-1}$. HRMS (EI) m/z calculated for $\mathrm{C}_{14} \mathrm{H}_{16} \mathrm{NO}_{2}\left[\mathrm{M}+\mathrm{H}-\mathrm{N}_{2}\right]^{+}: 230.1181$, found: 230.1188 . 


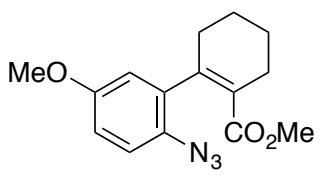

$16 \mathrm{~b}$

Styryl azide $16 \mathbf{b} .^{26}$ The general procedure was followed by using $0.086 \mathrm{~g}$ of 2-azidophenyl boronate $\mathbf{s 2 b}(0.315 \mathrm{mmol})$, $0.100 \mathrm{~g}$ of vinyl triflate s13a $(0.347 \mathrm{mmol}), 0.0462 \mathrm{~g}$ of $\mathrm{Pd}\left(\mathrm{PPh}_{3}\right)_{4}(0.0400 \mathrm{mmol}), 0.6 \mathrm{~mL}$ of a saturated aq. soln. of $\mathrm{NaHCO}_{3}$ and $4 \mathrm{~mL}$ of dimethoxyethane. Purification by MPLC (3:97 to 10:90 EtOAc:hexanes) afforded the product as a yellow solid $(0.046 \mathrm{~g}, 54 \%)$. The spectral data matched that reported by Driver and co-workers: ${ }^{26}{ }^{1} \mathrm{H} \mathrm{NMR}(500 \mathrm{MHz}$, $\left.\mathrm{CDCl}_{3}\right) \delta 7.02(\mathrm{~d}, J=7.5 \mathrm{~Hz}, 1 \mathrm{H}), 6.82(\mathrm{dd}, J=9.0 \mathrm{~Hz}, 2.0 \mathrm{~Hz}, 1 \mathrm{H}), 6.54(\mathrm{~d}, J=2.5 \mathrm{~Hz}, 1 \mathrm{H}), 3.76(\mathrm{~s}, 3 \mathrm{H}), 3.47(\mathrm{~s}, 3 \mathrm{H})$, $2.44(\mathrm{~m}, 4 \mathrm{H}), 1.73(\mathrm{~m}, 4 \mathrm{H}) ;{ }^{13} \mathrm{C}$ NMR $\left(125 \mathrm{MHz}, \mathrm{CDCl}_{3}\right) \delta 168.4(\mathrm{C}), 156.7(\mathrm{C}), 144.9$ (C), 140.0 (C), 128.8 (C), 128.4 $(\mathrm{C}), 119.4(\mathrm{CH}), 113.9(\mathrm{CH}), 113.5(\mathrm{CH}), 55.6\left(\mathrm{CH}_{3}\right), 51.3\left(\mathrm{CH}_{3}\right), 32.8\left(\mathrm{CH}_{2}\right), 26.1\left(\mathrm{CH}_{2}\right), 22.2\left(\mathrm{CH}_{2}\right), 21.9\left(\mathrm{CH}_{2}\right)$; ATRFTIR (thin film): 2939, 2114, 1714, 1488, 1284, $1242 \mathrm{~cm}^{-1}$.

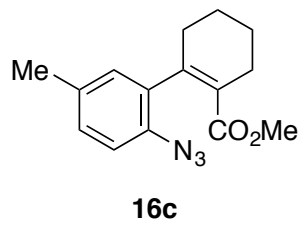

Styryl azide 16c. ${ }^{26}$ The general procedure was followed by using $0.455 \mathrm{~g}$ of 2-azidoarylboronate $\mathbf{s 2 c}(1.76 \mathrm{mmol}), 0.461$ $\mathrm{g}$ of vinyl triflate s13a $(1.60 \mathrm{mmol}), 0.199 \mathrm{~g}$ of $\mathrm{Pd}\left(\mathrm{PPh}_{3}\right)_{4}(0.16 \mathrm{mmol}), 3.2 \mathrm{~mL}$ of saturated aq. soln. of $\mathrm{NaHCO}_{3}$ and 20 $\mathrm{mL}$ of dimethoxyethane. Purification by MPLC (3:97 to 10:90 EtOAc: hexane) afforded the product as yellow oil (0.260 $\mathrm{g}, 55 \%)$. The spectral data matched that reported by Driver and co-workers: ${ }^{26}{ }^{1} \mathrm{H} \mathrm{NMR}\left(500 \mathrm{MHz}, \mathrm{CDCl}_{3}\right) \delta 7.08(\mathrm{~d}, J=$ $8.1 \mathrm{~Hz}, 1 \mathrm{H}), 7.01(\mathrm{~d}, J=8.1 \mathrm{~Hz}, 1 \mathrm{H}), 6.81(\mathrm{~s}, 1 \mathrm{H}), 3.46(\mathrm{~s}, 3 \mathrm{H}), 2.45(\mathrm{~s}, 3 \mathrm{H}), 2.30(\mathrm{~s}, 3 \mathrm{H}), 2.23(\mathrm{~m}, 1 \mathrm{H}), 1.74(\mathrm{~d}, J=3.7$ $\mathrm{Hz}, 4 \mathrm{H}) ;{ }^{13} \mathrm{C}$ NMR (125 MHz, $\left.\mathrm{CDCl}_{3}\right) \delta 168.4(\mathrm{C}), 145.3(\mathrm{C}), 135.6(\mathrm{C}), 134.3(\mathrm{C}), 133.5(\mathrm{C}), 129.0(\mathrm{CH}), 128.8(\mathrm{CH})$, $128.2(\mathrm{C}), 118.2(\mathrm{CH}), 51.2\left(\mathrm{CH}_{3}\right), 33.0\left(\mathrm{CH}_{2}\right), 26.1\left(\mathrm{CH}_{2}\right), 22.2\left(\mathrm{CH}_{2}\right), 22.0\left(\mathrm{CH}_{2}\right), 20.8\left(\mathrm{CH}_{3}\right)$. ATR-FTIR (thin film): 2937, 2858, 2110, 1707, $1229 \mathrm{~cm}^{-1}$; HRMS (EI) m/z calculated for $\mathrm{C}_{15} \mathrm{H}_{18} \mathrm{NO}_{2}\left(\mathrm{M}+\mathrm{H}-\mathrm{N}_{2}\right)^{+}:$244.1338, found: 244.1332.

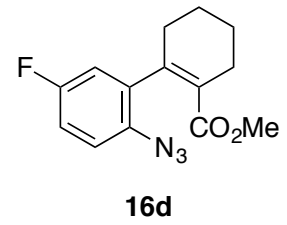

Styryl azide 16d. ${ }^{26}$ The general procedure was followed by using $0.246 \mathrm{~g}$ of 2-azidoarylboronate s2d $(0.93 \mathrm{mmol}), 0.245$ $\mathrm{g}$ of vinyl triflate s13a $(0.850 \mathrm{mmol}), 0.106 \mathrm{~g}$ of $\mathrm{Pd}\left(\mathrm{PPh}_{3}\right)_{4}(0.090 \mathrm{mmol}), 1.7 \mathrm{~mL}$ of saturated aq. soln. of $\mathrm{NaHCO}_{3}$ and 10 $\mathrm{mL}$ of dimethoxyethane. Purification by MPLC (3:97 to 10:90 EtOAc: hexane) afforded the product as yellow oil (0.185 $\mathrm{g}, 77 \%)$. The spectral data matched that reported by Driver and co-workers: ${ }^{26}{ }^{1} \mathrm{H} \mathrm{NMR}\left(500 \mathrm{MHz}, \mathrm{CDCl}_{3}\right) \delta 7.06(\mathrm{dd}, J=$ $8.8 \mathrm{~Hz}, 4.7 \mathrm{~Hz}, 1 \mathrm{H}), 6.97(\mathrm{td}, J=8.3 \mathrm{~Hz}, 2.9 \mathrm{~Hz}, 1 \mathrm{H}), 6.72(\mathrm{dd}, J=8.7 \mathrm{~Hz}, 2.8 \mathrm{~Hz}, 1 \mathrm{H}), 3.48(\mathrm{~s}, 3 \mathrm{H}), 2.44(\mathrm{~s}, 2 \mathrm{H}), 2.37$ $(\mathrm{s}, 1 \mathrm{H}), 2.24(\mathrm{dt}, J=25.0 \mathrm{~Hz}, 6.3 \mathrm{~Hz}, 1 \mathrm{H}), 1.73(\mathrm{~d}, J=4.1 \mathrm{~Hz}, 4 \mathrm{H}) ;{ }^{13} \mathrm{C} \mathrm{NMR}\left(125 \mathrm{MHz}, \mathrm{CDCl}_{3}\right) \delta 167.9(\mathrm{C}), 159.6(\mathrm{~d}$, $\left.J_{C F}=245.7 \mathrm{~Hz}, \mathrm{C}\right), 144.2(\mathrm{C}), 137.6\left(\mathrm{~d}, J_{C F}=8.8 \mathrm{~Hz}, \mathrm{C}\right), 132.1(\mathrm{C}), 128.8(\mathrm{C}), 119.6\left(\mathrm{~d}, J_{C F}=9.2 \mathrm{~Hz}, \mathrm{CH}\right), 115.4\left(\mathrm{~d}, J_{C F}=\right.$ $23.7 \mathrm{~Hz}, \mathrm{CH}), 114.7\left(\mathrm{~d}, J_{C F}=23.9 \mathrm{~Hz}, \mathrm{CH}\right), 51.3\left(\mathrm{CH}_{3}\right), 32.8\left(\mathrm{CH}_{2}\right), 26.0\left(\mathrm{CH}_{2}\right), 22.1\left(\mathrm{CH}_{2}\right), 21.9\left(\mathrm{CH}_{2}\right) ;{ }^{19} \mathrm{~F} \mathrm{NMR}(282$ $\left.\mathrm{MHz}, \mathrm{CDCl}_{3}\right) \delta-118.5$. ATR-FTIR (thin film): 2937, 2113, 1720, 1485, $1236 \mathrm{~cm}^{-1}$; HRMS (EI) m/z calculated for $\mathrm{C}_{14} \mathrm{H}_{15} \mathrm{NO}_{2} \mathrm{~F}\left(\mathrm{M}+\mathrm{H}-\mathrm{N}_{2}\right)^{+}: 248.1087$, found: 248.1084 . 


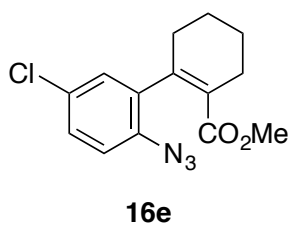

Styryl azide 16e. ${ }^{26}$ The general procedure was followed by using $0.060 \mathrm{~g}$ of 2-azidoarylboronate s2e $(0.22 \mathrm{mmol}), 0.055$ $\mathrm{g}$ of vinyl triflate s13a $(0.20 \mathrm{mmol}), 0.025 \mathrm{~g}$ of $\mathrm{Pd}\left(\mathrm{PPh}_{3}\right)_{4}(0.020 \mathrm{mmol}), 0.4 \mathrm{~mL}$ of saturated aq. soln. of $\mathrm{NaHCO}_{3}$ and 5 $\mathrm{mL}$ of dimethoxyethane. Purification by MPLC (3:97 to 10:90 EtOAc: hexane) afforded the product as yellow solid $(0.040 \mathrm{~g}, 71 \%)$. The spectral data matched that reported by Driver and co-workers: ${ }^{26}{ }^{1} \mathrm{H} \mathrm{NMR}\left(500 \mathrm{MHz}, \mathrm{CDCl}_{3}\right) \delta 7.23$ $(\mathrm{dd}, J=8.5 \mathrm{~Hz}, 2.4 \mathrm{~Hz}, 1 \mathrm{H}), 7.04(\mathrm{~d}, J=8.5 \mathrm{~Hz}, 1 \mathrm{H}), 6.98(\mathrm{~d}, J=2.3 \mathrm{~Hz}, 1 \mathrm{H}), 3.49(\mathrm{~s}, 3 \mathrm{H}), 2.44(\mathrm{~s}, 2 \mathrm{H}), 2.36(\mathrm{~s}, 1 \mathrm{H})$, $2.23(\mathrm{dt}, J=25.4 \mathrm{~Hz}, 6.1 \mathrm{~Hz}, 1 \mathrm{H}), 1.73(\mathrm{~s}, 4 \mathrm{H}) ;{ }^{13} \mathrm{C} \mathrm{NMR}\left(125 \mathrm{MHz}, \mathrm{CDCl}_{3}\right)$ d $167.9(\mathrm{C}), 144.1(\mathrm{C}), 137.4(\mathrm{C}), 135.0(\mathrm{C})$, $129.9(\mathrm{C}), 128.9(\mathrm{C}), 128.3(\mathrm{CH}), 128.0(\mathrm{CH}), 119.5(\mathrm{CH}), 51.4(\mathrm{CH} 3), 32.9\left(\mathrm{CH}_{2}\right), 26.0\left(\mathrm{CH}_{2}\right), 22.1\left(\mathrm{CH}_{2}\right), 21.9\left(\mathrm{CH}_{2}\right)$. ATR-FTIR (thin film): 2939, 2858, 2122, 2095, 1721, 1483, $1220 \mathrm{~cm}^{-1}$; HRMS (EI) m/z calculated for $\mathrm{C}_{14} \mathrm{H}_{15} \mathrm{NO}_{2} \mathrm{Cl}(\mathrm{M}+$ $\left.\mathrm{H}-\mathrm{N}_{2}\right)^{+}: 264.0791$, found: 264.0792 .

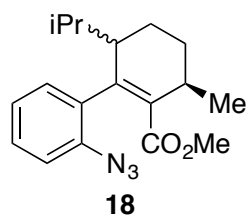

Styryl azide 18. The general procedure was followed by using $0.164 \mathrm{~g}$ of 2-azidophenyl boronate s2a $(0.670 \mathrm{mmol})$, $0.209 \mathrm{~g}$ of vinyl triflate s13b $(0.610 \mathrm{mmol}), 0.076 \mathrm{~g}$ of $\mathrm{Pd}\left(\mathrm{PPh}_{3}\right)_{4}(0.060 \mathrm{mmol}), 1.2 \mathrm{~mL}$ of a saturated aq. soln. of $\mathrm{NaHCO}_{3}$ and $10 \mathrm{~mL}$ of dimethoxyethane. Purification by MPLC (3:97 to 10:90 EtOAc:hexanes) afforded the product, a yellow oil, as a 3:1 mixture of diastereomers with each diastereomer appearing as a mixture of two rotamers $(0.106 \mathrm{~g}$, 56\%): ${ }^{1} \mathrm{H}$ NMR $\left(500 \mathrm{MHz}, \mathrm{CDCl}_{3}\right) \delta 7.27(\mathrm{t}, J=6.6 \mathrm{~Hz}, 1 \mathrm{H}), 7.07(\mathrm{~m}, 3 \mathrm{H}), 3.36(\mathrm{~s}, 3 \mathrm{H}), 2.68(\mathrm{~m}, 2 \mathrm{H}), 1.85(\mathrm{~m}, 1 \mathrm{H}), 1.65$ $(\mathrm{m}, 2 \mathrm{H}), 1.21(\mathrm{~d}, J=6.5 \mathrm{~Hz}, 3 \mathrm{H}), 1.06(\mathrm{~d}, J=7.0 \mathrm{~Hz}, 1 \mathrm{H}), 1.00(\mathrm{~m}, 1 \mathrm{H}), 0.86(\mathrm{~d}, J=7.0 \mathrm{~Hz}, 3 \mathrm{H}), 0.74(\mathrm{~d}, J=7.0 \mathrm{~Hz}$, $3 \mathrm{H}) ;{ }^{13} \mathrm{C}$ NMR $\left(125 \mathrm{MHz}, \mathrm{CDCl}_{3}\right) \delta 169.8(\mathrm{C}), 144.3(\mathrm{C}), 136.5(\mathrm{C}), 132.9(\mathrm{C}), 131.0(\mathrm{CH}), 128.2(\mathrm{CH}), 124.7(\mathrm{C}), 123.9$ $(\mathrm{CH}), 118.1(\mathrm{CH}), 51.1\left(\mathrm{CH}_{3}\right), 44.0(\mathrm{CH}), 30.6(\mathrm{CH}), 28.7\left(\mathrm{CH}_{2}\right), 28.3(\mathrm{CH}), 21.2\left(\mathrm{CH}_{3}\right), 20.4\left(\mathrm{CH}_{3}\right), 17.0\left(\mathrm{CH}_{2}\right), 16.6$ $\left(\mathrm{CH}_{3}\right)$. ATR-FTIR (thin film): 2958, 2871, 2121, 2088, 1722, $1210 \mathrm{~cm}^{-1}$; HRMS (ES) $\mathrm{m} / z$ calculated for $\mathrm{C}_{18} \mathrm{H}_{24} \mathrm{NO}_{2}[\mathrm{M}+$ $\left.\mathrm{H}-\mathrm{N}_{2}\right]^{+}:$286.1807, found: 286.1816. Characteristic data for minor diastereomer: ${ }^{1} \mathrm{H} \mathrm{NMR}\left(500 \mathrm{MHz}, \mathrm{CDCl}_{3}\right) \delta 3.45(\mathrm{~s}$, $3 \mathrm{H}), 2.50-2.41(\mathrm{~m}, 2 \mathrm{H}), 1.28(\mathrm{~d}, J=7.0 \mathrm{~Hz}, 3 \mathrm{H}) ;{ }^{13} \mathrm{C} \mathrm{NMR}\left(125 \mathrm{MHz}, \mathrm{CDCl}_{3}\right) \delta 52.1\left(\mathrm{CH}_{3}\right), 42.9(\mathrm{CH})$.

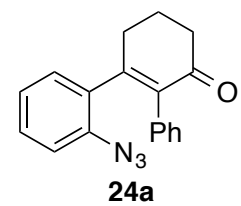

Styryl azide 24a. The general procedure was followed by using $0.104 \mathrm{~g}$ of 2 -azidophenyl boronate $\mathbf{~ s 2 a}(0.420 \mathrm{mmol})$, $0.123 \mathrm{~g}$ of vinyl triflate $\mathbf{s 1 0 a}(0.380 \mathrm{mmol}), 0.048 \mathrm{~g}$ of $\mathrm{Pd}\left(\mathrm{PPh}_{3}\right)_{4}(0.040 \mathrm{mmol}), 0.8 \mathrm{~mL}$ of a saturated aq. soln. of $\mathrm{NaHCO}_{3}$ and $4 \mathrm{~mL}$ of dimethoxyethane. Purification by MPLC (10:90 to 50:50 EtOAc:hexanes) afforded the product as yellow solid $(0.075 \mathrm{~g}, 68 \%):{ }^{1} \mathrm{H}$ NMR $\left(500 \mathrm{MHz}, \mathrm{CDCl}_{3}\right) \delta 7.18(\mathrm{t}, J=7.7 \mathrm{~Hz}, 1 \mathrm{H}), 7.11(\mathrm{~m}, 3 \mathrm{H}), 7.03(\mathrm{~d}, J=8.0 \mathrm{~Hz}$, $1 \mathrm{H}), 6.95(\mathrm{~d}, J=7.2 \mathrm{~Hz}, 2 \mathrm{H}), 6.91(\mathrm{~d}, J=7.4 \mathrm{~Hz}, 1 \mathrm{H}), 6.87(\mathrm{~d}, J=7.5 \mathrm{~Hz}, 1 \mathrm{H}), 2.82(\mathrm{~s}, 1 \mathrm{H}), 2.69(\mathrm{t}, J=6.5 \mathrm{~Hz}, 3 \mathrm{H})$, $2.24(\mathrm{~s}, 2 \mathrm{H}) ;{ }^{13} \mathrm{C}$ NMR $\left(125 \mathrm{MHz}, \mathrm{CDCl}_{3}\right) \delta 198.1(\mathrm{C}), 156.2(\mathrm{C}), 139.2(\mathrm{C}), 136.7(\mathrm{C}), 135.3(\mathrm{C}), 133.0(\mathrm{C}), 130.1(\mathrm{CH})$, $129.4(\mathrm{CH}), 129.0(\mathrm{CH}), 127.4(\mathrm{CH}), 126.9(\mathrm{CH}), 124.6(\mathrm{CH}), 118.3(\mathrm{CH}), 38.4\left(\mathrm{CH}_{2}\right), 32.7\left(\mathrm{CH}_{2}\right), 22.7\left(\mathrm{CH}_{2}\right)$; ATRFTIR (thin film): 2938, 2128, 2084, 1668, 1485, $1329 \mathrm{~cm}^{-1}$. HRMS (ES) $\mathrm{m} / z$ calculated for $\mathrm{C}_{18} \mathrm{H}_{15} \mathrm{~N}_{3} \mathrm{ONa}[\mathrm{M}+\mathrm{Na}]^{+}$: 312.1113, found: 312.1113 . 


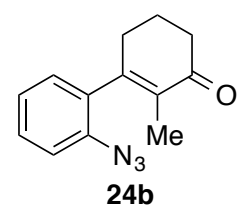

Styryl azide 24b. The general procedure was followed by using $0.200 \mathrm{~g}$ of 2 -azidophenyl boronate $\mathbf{s 2 a}(0.820 \mathrm{mmol})$, $0.191 \mathrm{~g}$ of vinyl triflate $\mathbf{s 1 0 b}(0.740 \mathrm{mmol}), 0.085 \mathrm{~g}$ of $\mathrm{Pd}\left(\mathrm{PPh}_{3}\right)_{4}(0.070 \mathrm{mmol}), 1.5 \mathrm{~mL}$ of a saturated aq. soln. of $\mathrm{NaHCO}_{3}$ and $10 \mathrm{~mL}$ of dimethoxyethane. Purification by MPLC (10:90 to 50:50 EtOAc:hexanes) afforded the product as yellow solid $(0.096 \mathrm{~g}, 57 \%):{ }^{1} \mathrm{H}$ NMR $\left(500 \mathrm{MHz}, \mathrm{CDCl}_{3}\right) \delta 7.36(\mathrm{t}, J=7.7 \mathrm{~Hz}, 1 \mathrm{H}), 7.20(\mathrm{~d}, J=7.9 \mathrm{~Hz}, 1 \mathrm{H}), 7.16(\mathrm{~d}, J=$ $7.5 \mathrm{~Hz}, 1 \mathrm{H}), 7.04(\mathrm{dd}, J=7.6 \mathrm{~Hz}, 1.2 \mathrm{~Hz}, 1 \mathrm{H}), 2.61-2.43(\mathrm{~m}, 4 \mathrm{H}), 2.09(\mathrm{~m}, 2 \mathrm{H}), 1.56(\mathrm{~s}, 3 \mathrm{H}) ;{ }^{13} \mathrm{C} \mathrm{NMR}(125 \mathrm{MHz}$, $\left.\mathrm{CDCl}_{3}\right) \delta 199.5(\mathrm{C}), 153.9(\mathrm{C}), 136.4(\mathrm{C}), 133.3(\mathrm{C}), 133.0(\mathrm{C}), 129.1(\mathrm{CH}), 128.5(\mathrm{CH}), 125.0(\mathrm{CH}), 118.7(\mathrm{CH}), 37.9$ $\left(\mathrm{CH}_{2}\right), 32.4\left(\mathrm{CH}_{2}\right), 22.8\left(\mathrm{CH}_{2}\right), 12.7\left(\mathrm{CH}_{3}\right)$; ATR-FTIR (thin film): 2924, 2866, 2112, 1665, $1284 \mathrm{~cm}^{-1}$. HRMS (ES) $\mathrm{m} / z$ calculated for $\mathrm{C}_{13} \mathrm{H}_{14} \mathrm{NO}\left[\mathrm{M}+\mathrm{H}-\mathrm{N}_{2}\right]^{+}: 200.1075$, found: 200.1076 .

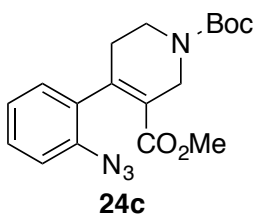

1-tert-Butyl-3-methyl-4-(2-azidophenyl)-5,6-dihydropyridine-1,3(2H)-dicarboxylate $24 c$. $^{26}$ The general procedure was followed by using $0.147 \mathrm{~g}$ of 2-azidophenylboronate s2a $(0.60 \mathrm{mmol}), 0.212 \mathrm{~g}$ of vinyl triflate $\mathbf{s 1 4}(0.540 \mathrm{mmol})$, $0.067 \mathrm{~g}$ of $\mathrm{Pd}\left(\mathrm{PPh}_{3}\right)_{4}(0.050 \mathrm{mmol}), 1.1 \mathrm{~mL}$ of a saturated aq. soln. of $\mathrm{NaHCO}_{3}$ and $6 \mathrm{~mL}$ of dimethoxyethane. Purification by MPLC (3:97 to 10:90 EtOAc:hexanes) afforded the product as a green solid $(0.155 \mathrm{~g}, 79 \%)$. The spectral data matched that reported by Driver and co-workers: ${ }^{26}{ }^{1} \mathrm{H} \mathrm{NMR}\left(500 \mathrm{MHz}, \mathrm{CDCl}_{3}\right) \delta 7.31(\mathrm{td}, J=7.7 \mathrm{~Hz}, 1.1 \mathrm{~Hz}, 1 \mathrm{H})$, $7.15(\mathrm{~d}, J=7.9 \mathrm{~Hz}, 1 \mathrm{H}), 7.10(\mathrm{t}, J=7.5 \mathrm{~Hz}, 1 \mathrm{H}), 6.98(\mathrm{dd}, J=7.6 \mathrm{~Hz}, 1.2 \mathrm{~Hz}, 1 \mathrm{H}), 4.24(\mathrm{~m}, 2 \mathrm{H}), 3.71(\mathrm{~s}, 1 \mathrm{H}), 3.49(\mathrm{~s}$, 4H), $2.43(\mathrm{~s}, 2 \mathrm{H}), 1.49(\mathrm{~s}, 9 \mathrm{H}) ;{ }^{13} \mathrm{C}$ NMR $\left(125 \mathrm{MHz}, \mathrm{CDCl}_{3}\right) \delta 154.6(\mathrm{C}), 145.5(\mathrm{C}), 136.2(\mathrm{C}), 133.9(\mathrm{C}), 128.7(\mathrm{CH})$, $128.3(\mathrm{CH}), 124.7(\mathrm{CH}), 118.3(\mathrm{CH}), 80.1(\mathrm{C}), 51.5\left(\mathrm{CH}_{3}\right), 43.7\left(\mathrm{CH}_{2}\right), 39.1\left(\mathrm{CH}_{2}\right), 32.7\left(\mathrm{CH}_{2}\right), 28.5\left(\mathrm{CH}_{3}\right)$; only visible peaks. ATR-FTIR (thin film): 2978, 2127, 2094, 1694, $1417 \mathrm{~cm}^{-1}$; HRMS (EI) m/z calculated for $\mathrm{C}_{18} \mathrm{H}_{23} \mathrm{~N}_{4} \mathrm{O}_{4}[\mathrm{M}+\mathrm{H}]^{+}$: 359.1719, found: 359.1714 .

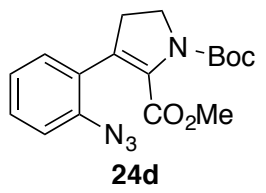

Styryl azide 24d. The general procedure was followed by using $0.094 \mathrm{~g}$ of 2-azidophenyl boronate $\mathbf{s 2 a}(0.38 \mathrm{mmol})$, $0.130 \mathrm{~g}$ of vinyl triflate $\mathbf{s 1 0 c}(0.340 \mathrm{mmol}), 0.040 \mathrm{~g}$ of $\mathrm{Pd}\left(\mathrm{PPh}_{3}\right)_{4}(0.030 \mathrm{mmol}), 0.6 \mathrm{~mL}$ of a saturated aq. soln. of $\mathrm{NaHCO}_{3}$ and $5 \mathrm{~mL}$ of dimethoxyethane. Purification by MPLC (10:90 to 50:50 EtOAc:hexanes) afforded the product as a yellow oil $(0.079 \mathrm{~g}, 66 \%)$ : ${ }^{1} \mathrm{H}$ NMR $\left(500 \mathrm{MHz}, \mathrm{CDCl}_{3}\right) \delta 7.30(\mathrm{t}, J=7.7 \mathrm{~Hz}, 1 \mathrm{H}), 7.17(\mathrm{~d}, J=7.6 \mathrm{~Hz}, 1 \mathrm{H}), 7.13(\mathrm{~d}, J=$ $8.0 \mathrm{~Hz}, 1 \mathrm{H}), 7.07(\mathrm{t}, J=7.5 \mathrm{~Hz}, 1 \mathrm{H}), 3.97(\mathrm{t}, J=9.2 \mathrm{~Hz}, 2 \mathrm{H}), 3.65(\mathrm{~s}, 3 \mathrm{H}), 2.99(\mathrm{t}, J=9.2 \mathrm{~Hz}, 2 \mathrm{H}), 1.44(\mathrm{~s}, 9 \mathrm{H}) ;{ }^{13} \mathrm{C}$ NMR (125 MHz, $\left.\mathrm{CDCl}_{3}\right) \delta 171.1(\mathrm{C}), 163.3(\mathrm{C}), 152.2(\mathrm{C}), 137.8(\mathrm{C}), 132.2(\mathrm{C}), 129.9(\mathrm{CH}), 129.2(\mathrm{CH}), 126.2(\mathrm{C})$, 124.6 (CH), $118.5(\mathrm{CH}), 81.3(\mathrm{C}), 52.0\left(\mathrm{CH}_{3}\right), 47.4\left(\mathrm{CH}_{2}\right), 33.4\left(\mathrm{CH}_{2}\right), 28.2\left(\mathrm{CH}_{3}\right)$. ATR-FTIR (thin film): 2980,2926 , 2126, 2093, 1737, 1698, 1392, $1264 \mathrm{~cm}^{-1}$; HRMS (ES) $\mathrm{m} / z$ calculated for $\mathrm{C}_{17} \mathrm{H}_{20} \mathrm{~N}_{4} \mathrm{O}_{4} \mathrm{Na}[\mathrm{M}+\mathrm{Na}]^{+}$: 367.1382, found: 367.1381 . 


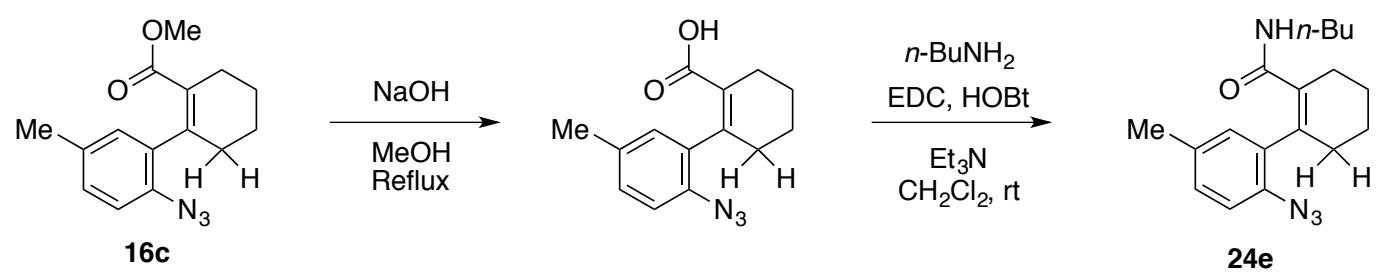

Styryl azide 24e. Compound 24e was prepared from styryl azide 16c using the following two-step procedure. To a 10 $\mathrm{mL}$ round bottom flask fitted with reflux condenser was added $0.035 \mathrm{~g}$ of styryl azide $16 \mathbf{c}(0.128 \mathrm{mmol})$ in $10 \mathrm{~mL}$ of methanol. To the resulting solution was added $0.031 \mathrm{~g}$ of $\mathrm{NaOH}(0.773 \mathrm{mmol})$, and the mixture was refluxed for 8 hours. The reaction mixture was cooled to room temperature and concentrated in vacuo. The residue was diluted with $20 \mathrm{~mL}$ of EtOAc. The diluted reaction mixture was taken in a separatory funnel and washed up with $10 \mathrm{~mL}$ of $1 \mathrm{~N} \mathrm{HCl,} 2 \times 10 \mathrm{~mL}$ of water followed by $10 \mathrm{~mL}$ of brine. The resulting organic phase was dried over $\mathrm{Na}_{2} \mathrm{SO}_{4}$, filtered and the filtrate was concentrated in vacuo. The crude carboxylic acid product $(0.035 \mathrm{~g})$ was used in the next step without further purification.

To a $10 \mathrm{~mL}$ round bottom flask fitted with nitrogen balloon was added $0.035 \mathrm{~g}$ of the crude carboxylic acid $(0.136 \mathrm{mmol})$ in $10 \mathrm{~mL}$ of dichloromethane. $N$-(3-Dimethylaminopropyl)- $N$ '-ethylcarbodiimide (EDC, $0.042 \mathrm{~g} 0.270 \mathrm{mmol}$ ), $0.027 \mathrm{~g}$ of HOBt $(0.204 \mathrm{mmol}), 0.028 \mathrm{~mL}$ of $\mathrm{Et}_{3} \mathrm{~N}(0.204 \mathrm{mmol})$ and $0.016 \mathrm{~mL}$ of $n$-butyl amine $(0.163 \mathrm{mmol})$ were subsequently added. After 3 hours, the reaction mixture was diluted with $10 \mathrm{~mL}$ of $\mathrm{CH}_{2} \mathrm{Cl}_{2}$, and the mixture was extracted with $2 \times 10$ $\mathrm{mL}$ of water followed by $10 \mathrm{~mL}$ of brine. The resulting organic phase was dried over $\mathrm{Na}_{2} \mathrm{SO}_{4}$, filtered and the filtrate was concentrated in vacuo. Purification by MPLC (3:97 to 10:90 EtOAc:hexanes) afforded the product as a white solid (0.007 g, 17\%): ${ }^{1} \mathrm{H}$ NMR $\left(500 \mathrm{MHz}, \mathrm{CDCl}_{3}\right) \delta 7.11(\mathrm{dd}, J=8.0 \mathrm{~Hz}, 1.5 \mathrm{~Hz}, 1 \mathrm{H}), 7.03(\mathrm{~d}, J=8.0 \mathrm{~Hz}, 1 \mathrm{H}), 6.91(\mathrm{~d}, J=1.0 \mathrm{~Hz}$, $1 \mathrm{H}), 5.26(\mathrm{~s}, 1 \mathrm{H}), 3.09-2.85(\mathrm{~m}, 2 \mathrm{H}), 2.53-2.36(\mathrm{~m}, 2 \mathrm{H}), 2.27(\mathrm{~s}, 3 \mathrm{H}), 2.04(\mathrm{~m}, 2 \mathrm{H}), 1.71(\mathrm{~m}, 4 \mathrm{H}), 0.94(\mathrm{~m}, 4 \mathrm{H}), 0.74$ $(\mathrm{t}, J=7.0 \mathrm{~Hz}, 3 \mathrm{H}) ;{ }^{13} \mathrm{C} \mathrm{NMR}\left(125 \mathrm{MHz}, \mathrm{CDCl}_{3}\right) \delta 170.3(\mathrm{C}), 136.0(\mathrm{C}), 135.2(\mathrm{C}), 134.3(\mathrm{C}), 134.2(\mathrm{C}), 134.0(\mathrm{C}), 130.5$ $(\mathrm{CH}), 129.3(\mathrm{CH}), 118.0(\mathrm{CH}), 38.7\left(\mathrm{CH}_{2}\right), 31.4\left(\mathrm{CH}_{2}\right), 31.3\left(\mathrm{CH}_{2}\right), 26.4\left(\mathrm{CH}_{2}\right), 22.5\left(\mathrm{CH}_{2}\right), 22.0\left(\mathrm{CH}_{2}\right), 20.7\left(\mathrm{CH}_{3}\right), 19.8$ $\left(\mathrm{CH}_{2}\right), 13.7\left(\mathrm{CH}_{3}\right)$; ATR-FTIR (thin film): 3483, 3052, 2931, 2861, 2253, 2130, 1633, $1490 \mathrm{~cm}^{-1}$.

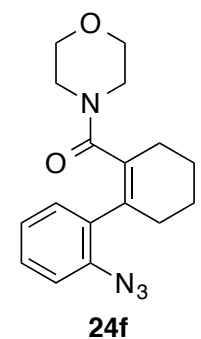

Styryl azide 24f. The general procedure was followed by using $0.176 \mathrm{~g}$ of 2 -azidophenyl boronate $\mathbf{s 2 a}(0.700 \mathrm{mmol})$, $0.227 \mathrm{~g}$ of vinyl triflate $\mathbf{s 1 2 a}(0.650 \mathrm{mmol}), 0.075 \mathrm{~g}$ of $\mathrm{Pd}\left(\mathrm{PPh}_{3}\right)_{4}(0.070 \mathrm{mmol}), 1.3 \mathrm{~mL}$ of a saturated aq. soln. of $\mathrm{NaHCO}_{3}$ and $10 \mathrm{~mL}$ of dimethoxyethane. Purification by MPLC (10:90 to 80:20 EtOAc:hexanes) afforded the product as a yellow oil $(0.200 \mathrm{~g}, 98 \%):{ }^{1} \mathrm{H}$ NMR $\left(500 \mathrm{MHz}, \mathrm{CDCl}_{3}\right) \delta 7.28(\mathrm{t}, J=7.5 \mathrm{~Hz}, 1 \mathrm{H}), 7.19(\mathrm{~d}, J=7.1 \mathrm{~Hz}, 1 \mathrm{H}), 7.12(\mathrm{~d}, J=$ $7.9 \mathrm{~Hz}, 1 \mathrm{H}), 7.06(\mathrm{t}, J=7.4 \mathrm{~Hz}, 1 \mathrm{H}), 3.48(\mathrm{~m}, 3 \mathrm{H}), 3.31(\mathrm{~m}, 3 \mathrm{H}), 2.98(\mathrm{~m}, 2 \mathrm{H}), 2.56(\mathrm{~m}, 2 \mathrm{H}), 2.07(\mathrm{~m}, 2 \mathrm{H}), 1.75(\mathrm{~s}, 4 \mathrm{H})$; ${ }^{13} \mathrm{C}$ NMR $\left(125 \mathrm{MHz}, \mathrm{CDCl}_{3}\right) \delta 170.6(\mathrm{C}), 137.0(\mathrm{C}), 134.3(\mathrm{C}), 133.2(\mathrm{C}), 131.8(\mathrm{C}), 130.9(\mathrm{CH}), 128.9(\mathrm{CH}), 125.0$ $(\mathrm{CH}), 118.2(\mathrm{CH}), 66.8\left(\mathrm{CH}_{2}\right), 66.6\left(\mathrm{CH}_{2}\right), 46.4\left(\mathrm{CH}_{2}\right), 41.3\left(\mathrm{CH}_{2}\right), 29.7\left(\mathrm{CH}_{2}\right), 27.2\left(\mathrm{CH}_{2}\right), 22.3\left(\mathrm{CH}_{2}\right), 21.9\left(\mathrm{CH}_{2}\right) ; \mathrm{ATR}-$ FTIR (thin film): 2922, 2865, 2120, 1620, $1433 \mathrm{~cm}^{-1}$. HRMS (ES) $\mathrm{m} / z$ calculated for $\mathrm{C}_{17} \mathrm{H}_{20} \mathrm{~N}_{4} \mathrm{O}_{2} \mathrm{Na}[\mathrm{M}+\mathrm{Na}]^{+}: 335.1484$, found: 335.1488 . 


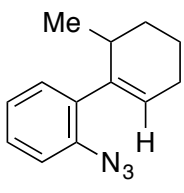

27

Styryl azide 27. The general procedure was followed by using $0.245 \mathrm{~g}$ of 2-azidophenyl boronate s2a $(0.100 \mathrm{mmol})$, $0.222 \mathrm{~g}$ of vinyl triflate $\mathbf{s 1 0 d}(0.910 \mathrm{mmol}), 0.105 \mathrm{~g}$ of $\mathrm{Pd}\left(\mathrm{PPh}_{3}\right)_{4}(0.0900 \mathrm{mmol}), 2 \mathrm{~mL}$ of a saturated aq. soln. of $\mathrm{NaHCO}_{3}$ and $15 \mathrm{~mL}$ of dimethoxyethane. Purification by MPLC (3:97 to 10:90 EtOAc:hexanes) afforded the product as a colorless oil $(0.112 \mathrm{~g}, 58 \%):{ }^{1} \mathrm{H}$ NMR $\left(500 \mathrm{MHz}, \mathrm{CDCl}_{3}\right) \delta 7.28(\mathrm{t}, J=8.3 \mathrm{~Hz}, 1 \mathrm{H}), 7.11(\mathrm{t}, J=9.6 \mathrm{~Hz}, 3 \mathrm{H}), 5.63(\mathrm{~s}, 1 \mathrm{H}), 2.78(\mathrm{~d}, J$ $=6.6 \mathrm{~Hz}, 1 \mathrm{H}), 2.18(\mathrm{t}, J=3.0 \mathrm{~Hz}, 2 \mathrm{H}), 1.95(\mathrm{~m}, 1 \mathrm{H}), 1.77(\mathrm{~m}, 1 \mathrm{H}), 1.68(\mathrm{~m}, 1 \mathrm{H}), 1.47(\mathrm{~m}, 1 \mathrm{H}), 0.84(\mathrm{~d}, J=7.1 \mathrm{~Hz}, 3 \mathrm{H})$; ${ }^{13} \mathrm{C}$ NMR $\left(125 \mathrm{MHz}, \mathrm{CDCl}_{3}\right) \delta 141.5(\mathrm{C}), 137.1(\mathrm{C}), 136.0(\mathrm{C}), 130.8(\mathrm{CH}), 127.8(\mathrm{CH}), 127.6(\mathrm{CH}), 124.6(\mathrm{CH}), 118.4$ $(\mathrm{CH}), 32.2(\mathrm{CH}), 31.4\left(\mathrm{CH}_{2}\right), 25.9\left(\mathrm{CH}_{2}\right), 20.0\left(\mathrm{CH}_{3}\right), 19.9\left(\mathrm{CH}_{2}\right)$; ATR-FTIR (thin film): 3019, 2926, 2868, 2115, 2082, $1482,1440,1283,748 \mathrm{~cm}^{-1}$. HRMS (EI) $\mathrm{m} / z$ calculated for $\mathrm{C}_{13} \mathrm{H}_{15} \mathrm{~N}_{3}[\mathrm{M}]^{+}: 213.1266$, found: 213.1275 .

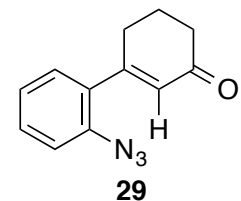

Styryl azide 29. The general procedure was followed by using $0.200 \mathrm{~g}$ of 2-azidophenyl boronate s2a $(0.820 \mathrm{mmol})$, $0.181 \mathrm{~g}$ of vinyl triflate $\mathbf{s 1 2 b}(0.740 \mathrm{mmol}), 0.043 \mathrm{~g}$ of $\mathrm{Pd}\left(\mathrm{PPh}_{3}\right)_{4}(0.040 \mathrm{mmol}), 1.5 \mathrm{~mL}$ of a saturated aq. soln. of $\mathrm{NaHCO}_{3}$ and $15 \mathrm{~mL}$ of dimethoxyethane. Purification by MPLC (3:97 to 10:90 EtOAc:hexanes) afforded the product as an orange oil $(0.117 \mathrm{~g}, 74 \%):{ }^{1} \mathrm{H}$ NMR $\left(500 \mathrm{MHz}, \mathrm{CDCl}_{3}\right) \delta 7.38(\mathrm{t}, J=7.7 \mathrm{~Hz}, 1 \mathrm{H}), 7.18(\mathrm{~d}, J=4.0 \mathrm{~Hz}, 2 \mathrm{H}), 7.14(\mathrm{~d}, J=$ $7.5 \mathrm{~Hz}, 1 \mathrm{H}), 6.07(\mathrm{~s}, 1 \mathrm{H}), 2.69(\mathrm{t}, J=5.9 \mathrm{~Hz}, 2 \mathrm{H}), 2.47(\mathrm{t}, J=6.7 \mathrm{~Hz}, 2 \mathrm{H}), 2.11$ (quintet, $J=6.4 \mathrm{~Hz}, 2 \mathrm{H}) ;{ }^{13} \mathrm{C} \mathrm{NMR}(125$ $\left.\mathrm{MHz}, \mathrm{CDCl}_{3}\right) \delta 199.5(\mathrm{C}), 160.4(\mathrm{C}), 137.0(\mathrm{C}), 132.6(\mathrm{C}), 130.1(\mathrm{CH}), 129.1(\mathrm{C}), 129.0(\mathrm{CH}), 125.0(\mathrm{CH}), 118.8(\mathrm{CH})$, $37.4\left(\mathrm{CH}_{2}\right), 30.4\left(\mathrm{CH}_{2}\right), 23.2\left(\mathrm{CH}_{2}\right)$; ATR-FTIR (thin film): 2945, 2867, 2115, 2086, 1666, 1482, $1285 \mathrm{~cm}^{-1}$. HRMS (ES) $m / z$ calculated for $\mathrm{C}_{12} \mathrm{H}_{12} \mathrm{NO}\left[\mathrm{M}+\mathrm{H}-\mathrm{N}_{2}\right]^{+}: 186.0919$, found: 186.0928 .

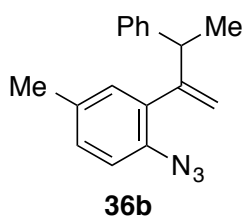

Styryl azide 36b. The general procedure was followed by using $0.058 \mathrm{~g}$ of 2 -azido-5-methylphenyl boronate $\mathbf{s 2 c}(0.22$ $\mathrm{mmol}), 0.068 \mathrm{~g}$ of vinyl triflate $\mathbf{~ s 1 1 a}(0.20 \mathrm{mmol}), 0.025 \mathrm{~g}$ of $\mathrm{Pd}\left(\mathrm{PPh}_{3}\right)_{4}(0.020 \mathrm{mmol}), 0.4 \mathrm{~mL}$ of a saturated aq. soln. of $\mathrm{NaHCO}_{3}$ and $8 \mathrm{~mL}$ of dimethoxyethane. Purification by MPLC (3:97 to 10:90 EtOAc:hexanes) afforded the product as a yellow oil $(0.046 \mathrm{~g}, 72 \%):{ }^{1} \mathrm{H}$ NMR $\left(500 \mathrm{MHz}, \mathrm{CDCl}_{3}\right) \delta 7.25(\mathrm{~m}, 2 \mathrm{H}), 7.20(\mathrm{~m}, 2 \mathrm{H}), 7.15(\mathrm{t}, J=7.5 \mathrm{~Hz}, 1 \mathrm{H}), 7.02(\mathrm{~d}, J=$ $8.0 \mathrm{~Hz}, 1 \mathrm{H}), 6.97(\mathrm{~d}, J=8.0 \mathrm{~Hz}, 1 \mathrm{H}), 6.73(\mathrm{~s}, 1 \mathrm{H}), 5.13(\mathrm{~s}, 1 \mathrm{H}), 5.02(\mathrm{~s}, 1 \mathrm{H}), 3.95(\mathrm{q}, J=7.0 \mathrm{~Hz}, 1 \mathrm{H}), 2.21(\mathrm{~s}, 3 \mathrm{H}), 1.41$ $(\mathrm{d}, J=7.1 \mathrm{~Hz}, 3 \mathrm{H}) ;{ }^{13} \mathrm{C}$ NMR $\left(125 \mathrm{MHz}, \mathrm{CDCl}_{3}\right) \delta 152.0(\mathrm{C}), 144.3(\mathrm{C}), 134.1(\mathrm{C}), 134.0(\mathrm{C}), 131.4(\mathrm{CH}), 128.9(\mathrm{CH})$, $128.1(\mathrm{CH}), 127.9(\mathrm{CH}), 126.2(\mathrm{CH}), 118.1(\mathrm{CH}), 114.8\left(\mathrm{CH}_{2}\right), 45.1(\mathrm{CH}), 20.8\left(\mathrm{CH}_{3}\right), 20.4\left(\mathrm{CH}_{3}\right)$, only peaks visible; ATR-FTIR (thin film): 2969, 2922, 211, 2072, 1489, $1293 \mathrm{~cm}^{-1}$. HRMS (EI) $\mathrm{m} / z$ calculated for $\mathrm{C}_{17} \mathrm{H}_{17} \mathrm{~N}_{3}[\mathrm{M}]^{+}: 263.1422$, found: 263.1421 . 


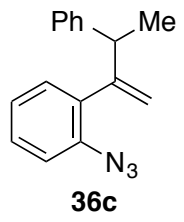

Styryl azide 36c. The general procedure was followed by using $0.381 \mathrm{~g}$ of 2 -azidophenyl boronate s2a (1.55 mmol), $0.396 \mathrm{~g}$ of vinyl triflate s11a $(1.40 \mathrm{mmol}), 0.174 \mathrm{~g}$ of $\mathrm{Pd}\left(\mathrm{PPh}_{3}\right)_{4}(0.140 \mathrm{mmol}), 3 \mathrm{~mL}$ of a saturated aq. soln. of $\mathrm{NaHCO}_{3}$ and $15 \mathrm{~mL}$ of dimethoxyethane. Purification by MPLC (3:97 to 10:90 EtOAc:hexanes) afforded the product as a colorless oil $(0.244 \mathrm{~g}, 69 \%):{ }^{1} \mathrm{H}$ NMR $\left(500 \mathrm{MHz}, \mathrm{CDCl}_{3}\right) \delta 7.31(\mathrm{~m}, 5 \mathrm{H}), 7.24(\mathrm{tt}, J=6.9 \mathrm{~Hz}, 1.9 \mathrm{~Hz}, 1 \mathrm{H}), 7.17(\mathrm{dd}, J=8.0 \mathrm{~Hz}, 0.6$ $\mathrm{Hz}, 1 \mathrm{H}), 7.03(\mathrm{~m}, 2 \mathrm{H}), 5.30(\mathrm{~s}, 1 \mathrm{H}), 5.16(\mathrm{~s}, 1 \mathrm{H}), 4.09(\mathrm{q}, J=7.1 \mathrm{~Hz}, 1 \mathrm{H}), 1.54(\mathrm{~d}, J=7.1 \mathrm{~Hz}, 3 \mathrm{H}) ;{ }^{13} \mathrm{C} \mathrm{NMR}(125 \mathrm{MHz}$, $\left.\mathrm{CDCl}_{3}\right)$ \& $151.9(\mathrm{C}), 144.4(\mathrm{C}), 137.1(\mathrm{C}), 135.4(\mathrm{C}), 131.0(\mathrm{CH}), 128.4(\mathrm{CH}), 128.3(\mathrm{CH}), 128.0(\mathrm{CH}), 126.3(\mathrm{CH}), 124.5$ $(\mathrm{CH}), 118.3(\mathrm{CH}), 115.0\left(\mathrm{CH}_{2}\right), 45.3(\mathrm{CH}), 20.5\left(\mathrm{CH}_{3}\right)$; ATR-FTIR (thin film): 2968, 2120, 2092, 1484, $1282 \mathrm{~cm}^{-1}$. HRMS (EI) $m / z$ calculated for $\mathrm{C}_{16} \mathrm{H}_{15} \mathrm{~N}_{3}[\mathrm{M}]^{+}: 249.1266$, found: 249.1270 .

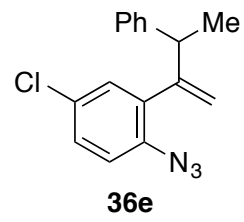

Styryl azide 36e. The general procedure was followed by using $0.160 \mathrm{~g}$ of 2-azido-5-chlorophenyl boronate s2e $(0.570$ $\mathrm{mmol}), 0.164 \mathrm{~g}$ of vinyl triflate s11a $(0.520 \mathrm{mmol}), 0.065 \mathrm{~g}$ of $\mathrm{Pd}\left(\mathrm{PPh}_{3}\right)_{4}(0.050 \mathrm{mmol}), 1.0 \mathrm{~mL}$ of a saturated aq. soln. of $\mathrm{NaHCO}_{3}$ and $10 \mathrm{~mL}$ of dimethoxyethane. Purification by MPLC (3:97 to 10:90 EtOAc:hexanes) afforded the product as a yellow oil $(0.079 \mathrm{~g}, 47 \%)$ : ${ }^{1} \mathrm{H}$ NMR $\left(500 \mathrm{MHz} \mathrm{CDCl}_{3}\right) \delta 7.26(\mathrm{~m}, 2 \mathrm{H}), 7.20-7.16(\mathrm{~m}, 6 \mathrm{H}), 7.02(\mathrm{~d}, J=8.5 \mathrm{~Hz}, 1 \mathrm{H})$, $6.91(\mathrm{~d}, J=2.5 \mathrm{~Hz}, 1 \mathrm{H}), 5.20(\mathrm{~s}, 1 \mathrm{H}), 5.06(\mathrm{~s}, 1 \mathrm{H}), 3.93(\mathrm{q}, J=7.0 \mathrm{~Hz}, 1 \mathrm{H}), 1.42(\mathrm{~d}, J=7.1 \mathrm{~Hz}, 3 \mathrm{H}) ;{ }^{13} \mathrm{C}$ NMR $(125$ $\left.\mathrm{MHz}, \mathrm{CDCl}_{3}\right) \delta 150.7(\mathrm{C}), 143.8(\mathrm{C}), 136.9(\mathrm{C}), 135.7(\mathrm{C}), 131.3(\mathrm{CH}), 130.6(\mathrm{CH}), 129.9(\mathrm{C}), 129.7(\mathrm{CH}), 128.3(\mathrm{CH})$, $126.4(\mathrm{CH}), 119.7(\mathrm{CH}), 115.7\left(\mathrm{CH}_{2}\right), 45.0(\mathrm{CH}), 20.3\left(\mathrm{CH}_{3}\right)$; ATR-FTIR (thin film): 2968, 2111, 2076, 1475, $1296 \mathrm{~cm}^{-1}$. HRMS (EI) $\mathrm{m} / z$ calculated for $\mathrm{C}_{16} \mathrm{H}_{14} \mathrm{ClN}_{3}[\mathrm{M}]^{+}: 283.0876$, found: 283.0870 .

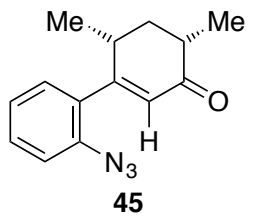

Styryl azide 45. The general procedure was followed by using $0.144 \mathrm{~g}$ of 2 -azidophenyl boronate s2a $(0.590 \mathrm{mmol})$, $0.145 \mathrm{~g}$ of vinyl triflate s10e $(0.530 \mathrm{mmol}), 0.067 \mathrm{~g}$ of $\mathrm{Pd}\left(\mathrm{PPh}_{3}\right)_{4}(0.050 \mathrm{mmol}), 1.0 \mathrm{~mL}$ of a saturated aq. soln. of $\mathrm{NaHCO}_{3}$ and $10 \mathrm{~mL}$ of dimethoxyethane. Purification by MPLC (3:97 to 10:90 EtOAc:hexanes) afforded the product, a yellow oil, as a 6:1 mixture of diastereomers $(0.097 \mathrm{~g}, 75 \%):{ }^{1} \mathrm{H} \mathrm{NMR}\left(500 \mathrm{MHz}, \mathrm{CDCl}_{3}\right) \delta 7.37(\mathrm{t}, J=7.6 \mathrm{~Hz}, 1 \mathrm{H}), 7.17$ $(\mathrm{d}, J=8.0 \mathrm{~Hz}, 1 \mathrm{H}), 7.14(\mathrm{t}, J=7.6 \mathrm{~Hz}, 1 \mathrm{H}), 7.10(\mathrm{dd}, J=7.6 \mathrm{~Hz}, 1.4 \mathrm{~Hz}, 1 \mathrm{H}), 5.92(\mathrm{~s}, 1 \mathrm{H}), 3.28(\mathrm{~m}, 1 \mathrm{H}), 2.55(\mathrm{~m}, 1 \mathrm{H})$, $2.15(\mathrm{dt}, J=13.2 \mathrm{~Hz}, 4.6 \mathrm{~Hz}, 1 \mathrm{H}), 1.54(\mathrm{td}, J=13.5 \mathrm{~Hz}, 11.2 \mathrm{~Hz}, 1 \mathrm{H}), 1.16(\mathrm{~d}, J=6.7 \mathrm{~Hz}, 3 \mathrm{H}), 0.86(\mathrm{~d}, J=7.1 \mathrm{~Hz}, 3 \mathrm{H})$; ${ }^{13} \mathrm{C}$ NMR $\left(125 \mathrm{MHz}, \mathrm{CDCl}_{3}\right) \delta 201.8(\mathrm{C}), 164.1(\mathrm{C}), 136.5(\mathrm{C}), 131.8(\mathrm{C}), 129.5(\mathrm{CH}), 129.4(\mathrm{CH}), 128.9(\mathrm{CH}), 125.0$ $(\mathrm{CH}), 118.2(\mathrm{CH}), 41.5(\mathrm{CH}), 40.7\left(\mathrm{CH}_{2}\right), 34.1(\mathrm{CH}), 19.9\left(\mathrm{CH}_{3}\right), 14.8\left(\mathrm{CH}_{3}\right)$. Characteristic data for minor diastereomer: ${ }^{1} \mathrm{H}$ NMR $\left(500 \mathrm{MHz}, \mathrm{CDCl}_{3}\right) \delta 5.84(\mathrm{~s}, 1 \mathrm{H}), 3.07(\mathrm{~m}, 1 \mathrm{H}), 1.92(\mathrm{~d}, J=6.5 \mathrm{~Hz}, 3 \mathrm{H}), 1.03(\mathrm{~d}, J=7.5 \mathrm{~Hz}, 3 \mathrm{H}) ;{ }^{13} \mathrm{C} \mathrm{NMR}$ $\left(125 \mathrm{MHz}, \mathrm{CDCl}_{3}\right) \delta 129.8(\mathrm{CH}), 127.7(\mathrm{CH}), 118.6(\mathrm{CH}), 37.8\left(\mathrm{CH}_{2}\right), 15.3\left(\mathrm{CH}_{3}\right)$. ATR-FTIR (thin film): 2962, 2929, 2852, 2123, 1672, $1284 \mathrm{~cm}^{-1}$. HRMS (ES) $\mathrm{m} / z$ calculated for $\mathrm{C}_{14} \mathrm{H}_{16} \mathrm{NO}\left[\mathrm{M}+\mathrm{H}-\mathrm{N}_{2}\right]^{+}: 214.1232$, found: 214.1242 . 


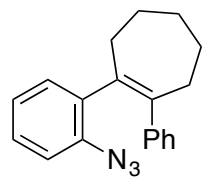

55

Styryl azide 55. The general procedure was followed by using $0.124 \mathrm{~g}$ of 2-azidophenyl boronate s2a $(0.510 \mathrm{mmol})$, $0.148 \mathrm{~g}$ of vinyl triflate $\mathbf{~} 15(0.460 \mathrm{mmol}), 0.053 \mathrm{~g}$ of $\mathrm{Pd}\left(\mathrm{PPh}_{3}\right)_{4}(0.046 \mathrm{mmol}), 0.8 \mathrm{~mL}$ of a saturated aq. soln. of $\mathrm{NaHCO}_{3}$ and $1.0 \mathrm{~mL}$ of dimethoxyethane. Purification by MPLC (3:97 to 10:90 EtOAc:hexanes) afforded the product as a yellow solid (0.096 g, 72\%): ${ }^{1} \mathrm{H}$ NMR $\left(500 \mathrm{MHz}, \mathrm{CDCl}_{3}\right) \delta 7.13-7.07(\mathrm{~m}, 3 \mathrm{H}), 7.04-7.01(\mathrm{~m}, 2 \mathrm{H}), 6.98(\mathrm{~d}, J=8.0 \mathrm{~Hz}, 2 \mathrm{H})$, $6.87-6.82(\mathrm{~m}, 2 \mathrm{H}), 2.77(\mathrm{~m}, 1 \mathrm{H}), 2.68(\mathrm{~m}, 1 \mathrm{H}), 2.52(\mathrm{~m}, 1 \mathrm{H}), 1.97-1.89(\mathrm{~m}, 4 \mathrm{H}), 1.76(\mathrm{~m}, 1 \mathrm{H}), 1.64(\mathrm{~m}, 1 \mathrm{H}) ;{ }^{13} \mathrm{C} \mathrm{NMR}$ $\left(125 \mathrm{MHz}, \mathrm{CDCl}_{3}\right) \delta 144.9(\mathrm{C}), 143.0(\mathrm{C}), 138.4(\mathrm{C}), 137.7(\mathrm{C}), 137.4(\mathrm{C}), 131.3(\mathrm{CH}), 128.1(\mathrm{CH}), 127.5(\mathrm{CH}), 127.3$ $(\mathrm{CH}), 125.8(\mathrm{CH}), 124.4(\mathrm{CH}), 118.3(\mathrm{CH}), 36.5\left(\mathrm{CH}_{2}\right), 36.4\left(\mathrm{CH}_{2}\right), 32.7\left(\mathrm{CH}_{2}\right), 27.1\left(\mathrm{CH}_{2}\right), 26.4\left(\mathrm{CH}_{2}\right)$. ATR-FTIR (thin film): 3053, 2920, 2840, 2120, 2088, 1572, 1481, 1440, 1283, 1272, $1091 \mathrm{~cm}^{-1}$; HRMS (ESI) $\mathrm{m} / \mathrm{z}$ calculated for $\mathrm{C}_{19} \mathrm{H}_{20} \mathrm{~N}$ $\left[\mathrm{M}+\mathrm{H}-\mathrm{N}_{2}\right]^{+}: 262.1596$, found: 262.1596 .

\section{B. Synthesis of Styryl Azides from Aniline.}

\section{General Procedure:}

Following the procedure of Zhang and Moses,${ }^{27}$ the azides were prepared. Yields were not optimized.<smiles>[R7]c1ccc(C(C)C(=C)c2cc([Tl])ccc2N)cc1</smiles><smiles>[R]c1cccc(C(=C)C(C)c2ccc([N+](=O)[O-])cc2)c1</smiles>

(s20)

To a cooled solution of aniline $\mathbf{s 1 6}$ in $\mathrm{MeCN}(0.1 \mathrm{M})$ was added dropwise $t$-BuONO (4 equiv) and $\mathrm{Me}_{3} \mathrm{SiN}_{3}(3$ equiv). The resulting solution was warmed to room temperature. After $1 \mathrm{~h}$, visualization of the reaction progress using TLC indicated the consumption of the starting material. De-ionized water was added to the reaction mixture. The mixture then was extracted with $2 \times 30 \mathrm{~mL}$ of $\mathrm{CH}_{2} \mathrm{Cl}_{2}$. The combined organic phases were washed with $20 \mathrm{~mL}$ of brine. The resulting organic phase was dried over $\mathrm{Na}_{2} \mathrm{SO}_{4}$, filtered, and the filtrate was concentrated in vacuo. Purification of the residue by MPLC (2:98 - 10:90 EtOAc: hexanes) afforded azide.

\section{Preparation of Styryl Azides:}

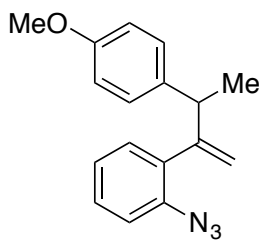

33a

Styryl azide 33a. The general procedure was followed by using $0.270 \mathrm{~g}$ of aniline s15a $(1.10 \mathrm{mmol}), 0.52 \mathrm{~mL}$ of $t$ BuONO (4.0 mmol), $0.41 \mathrm{~mL}$ of $\mathrm{Me}_{3} \mathrm{SiN}_{3}(3.0 \mathrm{mmol})$ and $10 \mathrm{~mL}$ of MeCN. Purification by MPLC (2:98 - 10:90 EtOAc:hexanes) afforded the product as a yellow oil $(0.224 \mathrm{~g}, 75 \%):{ }^{1} \mathrm{H} \mathrm{NMR}\left(500 \mathrm{MHz}, \mathrm{CDCl}_{3}\right) \delta 7.27(\mathrm{t}, J=7.6 \mathrm{~Hz}$, $1 \mathrm{H}), 7.18(\mathrm{~d}, J=8.5 \mathrm{~Hz}, 2 \mathrm{H}), 7.14(\mathrm{~d}, J=8.0 \mathrm{~Hz}, 1 \mathrm{H}), 7.02(\mathrm{t}, J=7.4 \mathrm{~Hz}, 1 \mathrm{H}), 6.98(\mathrm{~d}, J=7.5 \mathrm{~Hz}, 1 \mathrm{H}), 6.85(\mathrm{~d}, J=8.6$ $\mathrm{Hz}, 2 \mathrm{H}), 5.25(\mathrm{~s}, 1 \mathrm{H}), 5.10(\mathrm{~s}, 1 \mathrm{H}), 4.00(\mathrm{q}, J=7.0 \mathrm{~Hz}, 1 \mathrm{H}), 3.80(\mathrm{~s}, 3 \mathrm{H}), 1.48(\mathrm{~d}, J=7.1 \mathrm{~Hz}, 3 \mathrm{H}) ;{ }^{13} \mathrm{C} \mathrm{NMR}(125 \mathrm{MHz}$, $\left.\mathrm{CDCl}_{3}\right) \delta 158.0(\mathrm{C}), 152.2(\mathrm{C}), 137.0(\mathrm{C}), 136.4(\mathrm{C}), 135.4(\mathrm{C}), 130.9(\mathrm{CH}), 128.8(\mathrm{CH}), 128.3(\mathrm{CH}), 124.5(\mathrm{CH}), 118.3$ 
$(\mathrm{CH}), 114.6\left(\mathrm{CH}_{2}\right), 113.6(\mathrm{CH}), 55.2\left(\mathrm{CH}_{3}\right), 44.5(\mathrm{CH}), 20.5\left(\mathrm{CH}_{3}\right)$; ATR-FTIR (thin film): 2965, 2833, 2119, 2090, 1509, $1286,1244 \mathrm{~cm}^{-1}$. HRMS (ES) $\mathrm{m} / z$ calculated for $\mathrm{C}_{17} \mathrm{H}_{18} \mathrm{NO}\left[\mathrm{M}+\mathrm{H}-\mathrm{N}_{2}\right]^{+}: 252.1388$, found: 252.1394 .

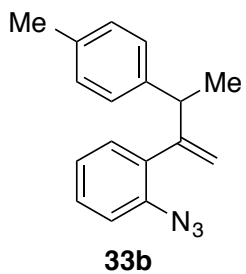

Styryl azide 33b. The general procedure was followed by using $0.310 \mathrm{~g}$ of $\mathbf{s 1 5 b}(1.30 \mathrm{mmol}), 0.66 \mathrm{~mL}$ of $t$-BuONO (5.2 $\mathrm{mmol}), 0.55 \mathrm{~mL}$ of $\mathrm{Me}_{3} \mathrm{SiN}_{3}(3.9 \mathrm{mmol})$ and $15 \mathrm{~mL}$ of MeCN. Purification by MPLC (2:98 - 10:90 EtOAc:hexanes) afforded the product as a colorless oil $(0.320 \mathrm{~g}, 93 \%)$ : ${ }^{1} \mathrm{H}$ NMR $\left(500 \mathrm{MHz}, \mathrm{CDCl}_{3}\right) \delta 7.30(\mathrm{t}, J=7.5 \mathrm{~Hz}, 1 \mathrm{H}), 7.16(\mathrm{~m}$, $5 \mathrm{H}), 7.03(\mathrm{~m}, 2 \mathrm{H}), 5.29(\mathrm{~s}, 1 \mathrm{H}), 5.14(\mathrm{~s}, 1 \mathrm{H}), 4.04(\mathrm{q}, J=7.0 \mathrm{~Hz}, 1 \mathrm{H}), 2.37(\mathrm{~s}, 3 \mathrm{H}), 1.52(\mathrm{~d}, J=7.1 \mathrm{~Hz}, 3 \mathrm{H}) ;{ }^{13} \mathrm{C} \mathrm{NMR}$ $\left(125 \mathrm{MHz}, \mathrm{CDCl}_{3}\right) \delta 152.0(\mathrm{C}), 141.3(\mathrm{C}), 137.0(\mathrm{C}), 135.7(\mathrm{C}), 135.5(\mathrm{C}), 131.0(\mathrm{CH}), 129.0(\mathrm{CH}), 128.3(\mathrm{CH}), 127.8$ $(\mathrm{CH}), 124.5(\mathrm{CH}), 118.3(\mathrm{CH}), 114.8\left(\mathrm{CH}_{2}\right), 44.9(\mathrm{CH}), 21.1\left(\mathrm{CH}_{3}\right), 20.6\left(\mathrm{CH}_{3}\right)$; ATR-FTIR (thin film): 2967, 2929, 2119 , $2089,1484,1282 \mathrm{~cm}^{-1}$. HRMS (EI) $\mathrm{m} / z$ calculated for $\mathrm{C}_{17} \mathrm{H}_{17} \mathrm{~N}_{3}[\mathrm{M}]^{+}: 263.1422$, found: 263.1429 .

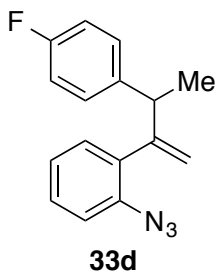

Styryl azide 33d. The general procedure was followed by using $0.194 \mathrm{~g}$ of aniline $\mathbf{s 1 5 c}(0.800 \mathrm{mmol}), 0.38 \mathrm{~mL}$ of $t$ BuONO (3.2 mmol), $0.32 \mathrm{~mL}$ of $\mathrm{Me}_{3} \mathrm{SiN}_{3}(2.4 \mathrm{mmol})$ and $15 \mathrm{~mL}$ of MeCN. Purification by MPLC (2:98 - 10:90 EtOAc:hexanes) afforded the product as a colorless oil $(0.150 \mathrm{~g}, 70 \%):{ }^{1} \mathrm{H}$ NMR $\left(500 \mathrm{MHz}, \mathrm{CDCl}_{3}\right) \delta 7.24(\mathrm{dd}, J=8.0$ $\mathrm{Hz}, 1.5 \mathrm{~Hz}, 1 \mathrm{H}), 7.15-7.09(\mathrm{~m}, 3 \mathrm{H}), 6.97(\mathrm{dt}, J=7.5 \mathrm{~Hz}, 1.0 \mathrm{~Hz}, 1 \mathrm{H}), 6.93-6.88(\mathrm{~m}, 3 \mathrm{H}), 5.17(\mathrm{~s}, 1 \mathrm{H}), 5.05(\mathrm{~s}, 1 \mathrm{H})$, $3.95(\mathrm{q}, J=7.0 \mathrm{~Hz}, 1 \mathrm{H}), 1.42(\mathrm{~d}, J=7.0 \mathrm{~Hz}, 3 \mathrm{H}) ;{ }^{13} \mathrm{C} \mathrm{NMR}\left(125 \mathrm{MHz}, \mathrm{CDCl}_{3}\right) \delta 161.3\left(\mathrm{~d}, J_{C F}=229.7 \mathrm{~Hz}, \mathrm{C}\right), 151.7(\mathrm{C})$, $135.0(\mathrm{C}), 139.9(\mathrm{C}), 136.9(\mathrm{C}), 130.8(\mathrm{CH}), 129.2\left(\mathrm{~d}, J_{C F}=7.4 \mathrm{~Hz}, \mathrm{CH}\right), 128.4(\mathrm{CH}), 124.4(\mathrm{CH}), 118.2(\mathrm{CH}), 114.9$ $(\mathrm{CH}), 114.8\left(\mathrm{~d}, J_{C F}=5.5 \mathrm{~Hz}, \mathrm{CH}\right), 44.3(\mathrm{CH}), 20.4\left(\mathrm{CH}_{3}\right) ;{ }^{19} \mathrm{~F}$ NMR $\left(282 \mathrm{MHz}, \mathrm{CDCl}_{3}\right) \delta-117.8$. ATR-FTIR (thin film): 2968, 2932, 2121, 2091, 1601, 1572, 1507, 1485, 1441, 1283, 1220, $1158 \mathrm{~cm}^{-1}$. HRMS (ESI) $\mathrm{m} / z$ calculated for $\mathrm{C}_{16} \mathrm{H}_{15} \mathrm{FN}$ $\left[\mathrm{M}-\mathrm{N}_{2}+\mathrm{H}\right]^{+}: 240.1189$, found: 240.1192 .

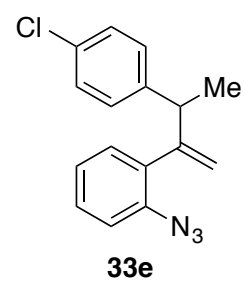

Styryl azide 33e. The general procedure was followed by using $0.310 \mathrm{~g}$ of $\mathbf{s 1 5 d}(1.20 \mathrm{mmol}), 0.61 \mathrm{~mL}$ of $t$-BuONO (4.8 $\mathrm{mmol}), 0.50 \mathrm{~mL}$ of $\mathrm{Me}_{3} \mathrm{SiN}_{3}(3.6 \mathrm{mmol})$ and $10 \mathrm{~mL}$ of MeCN. Purification by MPLC (2:98 - 10:90 EtOAc:hexanes) afforded the product as a colorless oil $(0.320 \mathrm{~g}, 94 \%):{ }^{1} \mathrm{H}$ NMR $\left(500 \mathrm{MHz}, \mathrm{CDCl}_{3}\right) \delta 7.25(\mathrm{~m}, 1 \mathrm{H}), 7.20(\mathrm{~m}, 2 \mathrm{H}), 7.10(\mathrm{~m}$, $3 \mathrm{H}), 6.99-6.96(\mathrm{~m}, 1 \mathrm{H}), 6.90-6.88(\mathrm{~m}, 1 \mathrm{H}), 5.18(\mathrm{~s}, 1 \mathrm{H}), 5.06(\mathrm{~s}, 1 \mathrm{H}), 3.96(\mathrm{q}, J=7.0 \mathrm{~Hz}, 1 \mathrm{H}), 1.41(\mathrm{~d}, J=7.5 \mathrm{~Hz}$, $3 \mathrm{H}) ;{ }^{13} \mathrm{C}$ NMR $\left(125 \mathrm{MHz}, \mathrm{CDCl}_{3}\right) \delta 151.4(\mathrm{C}), 142.9(\mathrm{C}), 136.9(\mathrm{C}), 134.9(\mathrm{C}), 131.8(\mathrm{C}), 130.8(\mathrm{CH}), 129.2(\mathrm{CH}), 128.4$ $(\mathrm{CH}), 128.3(\mathrm{CH}), 124.5(\mathrm{CH}), 118.2(\mathrm{CH}), 115.1\left(\mathrm{CH}_{2}\right), 44.5(\mathrm{CH}), 20.3\left(\mathrm{CH}_{3}\right)$; ATR-FTIR (thin film): 2973,2126 , 2091, 1484, $1279 \mathrm{~cm}^{-1}$. HRMS (EI) $\mathrm{m} / z$ calculated for $\mathrm{C}_{16} \mathrm{H}_{14} \mathrm{ClN}_{3}(\mathrm{M})^{+}: 283.0876$, found: 283.0878 . 


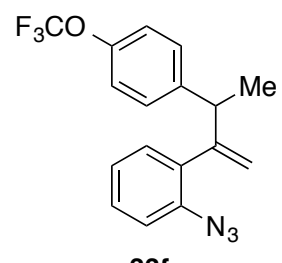

$33 f$

Styryl azide 33f. The general procedure was followed by using $0.100 \mathrm{~g}$ of aniline s15e $(0.325 \mathrm{mmol}), 0.15 \mathrm{~mL}$ of $t$ BuONO (1.30 mmol), $0.13 \mathrm{~mL}$ of $\mathrm{Me}_{3} \mathrm{SiN}_{3}(0.975 \mathrm{mmol})$ and $15 \mathrm{~mL}$ of MeCN. Purification by MPLC (2:98 - 10:90 EtOAc:hexanes) afforded the product, a colorless oil $(0.086 \mathrm{~g}, 80 \%):{ }^{1} \mathrm{H}$ NMR $\left(500 \mathrm{MHz}, \mathrm{CDCl}_{3}\right) \delta 7.27-7.25(\mathrm{~m}, 1 \mathrm{H})$, $7.20(\mathrm{~d}, J=8.5 \mathrm{~Hz}, 2 \mathrm{H}), 7.11-7.07(\mathrm{~m}, 3 \mathrm{H}), 6.98(\mathrm{dt}, J=7.5 \mathrm{~Hz}, 1.0 \mathrm{~Hz}, 1 \mathrm{H}), 6.89(\mathrm{dd}, J=8.0 \mathrm{~Hz}, 1.5 \mathrm{~Hz}, 1 \mathrm{H}), 5.17(\mathrm{~s}$, $1 \mathrm{H}), 5.06(\mathrm{~s}, 1 \mathrm{H}), 3.98(\mathrm{q}, J=7.0 \mathrm{~Hz}, 1 \mathrm{H}), 1.43(\mathrm{~d}, J=7.0 \mathrm{~Hz}, 3 \mathrm{H}) ;{ }^{13} \mathrm{C}$ NMR $\left(125 \mathrm{MHz}, \mathrm{CDCl}_{3}\right) \delta 151.4(\mathrm{C}), 147.6(\mathrm{C})$, $143.0(\mathrm{C}), 136.9(\mathrm{C}), 134.8(\mathrm{C}), 130.8(\mathrm{CH}), 129.1(\mathrm{CH}), 128.5(\mathrm{CH}), 124.5(\mathrm{CH}), 120.6(\mathrm{CH}), 118.2(\mathrm{CH}), 115.1(\mathrm{CH})$, $44.5(\mathrm{CH}), 20.3\left(\mathrm{CH}_{3}\right) ;{ }^{19} \mathrm{~F}$ NMR $\left(282 \mathrm{MHz}, \mathrm{CDCl}_{3}\right) \delta-58.33$. ATR-FTIR (thin film): 2969, 2122, 2093, 1633, 1506, $1485,1252,1219,1157 \mathrm{~cm}^{-1}$. HRMS (EI) $\mathrm{m} / z$ calculated for $\mathrm{C}_{17} \mathrm{H}_{15} \mathrm{~F}_{3} \mathrm{~N}_{3} \mathrm{O}(\mathrm{M}+\mathrm{H})^{+}:$306.1106, found: 306.1108 .

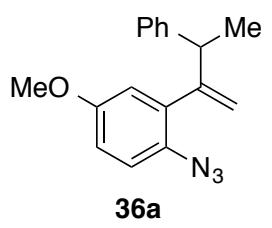

Styryl azide 36a. The general procedure was followed by using $0.085 \mathrm{~g}$ of $\mathbf{s 1 5 f}(0.34 \mathrm{mmol}), 0.170 \mathrm{~mL}$ of $t$-BuONO (1.34 mmol), $0.14 \mathrm{~mL}$ of $\mathrm{Me}_{3} \mathrm{SiN}_{3}(1.0 \mathrm{mmol})$ and $10 \mathrm{~mL}$ of MeCN. Purification by MPLC (2:98 - 10:90 EtOAc:hexanes) afforded the product as a colorless oil $(0.077 \mathrm{~g}, 83 \%):{ }^{1} \mathrm{H}$ NMR $\left(500 \mathrm{MHz}, \mathrm{CDCl}_{3}\right) \delta 7.26(\mathrm{~m}, 2 \mathrm{H}), 7.18$ $(\mathrm{m}, 2 \mathrm{H}), 7.15(\mathrm{t}, J=7.0 \mathrm{~Hz}, 1 \mathrm{H}), 7.00(\mathrm{~d}, J=8.5 \mathrm{~Hz}, 1 \mathrm{H}), 6.77(\mathrm{dd}, J=8.5 \mathrm{~Hz}, 3.0 \mathrm{~Hz}, 1 \mathrm{H}), 6.42(\mathrm{~d}, J=3.0 \mathrm{~Hz}, 1 \mathrm{H})$, $5.19(\mathrm{~s}, 1 \mathrm{H}), 5.06(\mathrm{~s}, 1 \mathrm{H}), 3.95(\mathrm{q}, J=7.5 \mathrm{~Hz}, 1 \mathrm{H}), 3.66(\mathrm{~s}, 3 \mathrm{H}), 1.43(\mathrm{~d}, J=7.0 \mathrm{~Hz}, 3 \mathrm{H}) ;{ }^{13} \mathrm{C} \mathrm{NMR}\left(125 \mathrm{MHz}, \mathrm{CDCl}_{3}\right) \delta$ $156.3(\mathrm{C}), 151.6(\mathrm{C}), 144.2(\mathrm{C}), 136.3(\mathrm{C}), 129.4(\mathrm{C}), 128.2(\mathrm{CH}), 127.9(\mathrm{CH}), 126.2(\mathrm{CH}), 119.2(\mathrm{CH}), 116.2(\mathrm{CH})$, $114.9\left(\mathrm{CH}_{2}\right), 113.8(\mathrm{CH}), 55.5\left(\mathrm{CH}_{3}\right), 45.2(\mathrm{CH}), 20.2\left(\mathrm{CH}_{3}\right)$; ATR-FTIR (thin film): 2966, 2834, 2115, 2074, 1488,1286 $\mathrm{cm}^{-1}$; HRMS (ES) $\mathrm{m} / z$ calculated for $\mathrm{C}_{17} \mathrm{H}_{18} \mathrm{NO}\left[\mathrm{M}+\mathrm{H}-\mathrm{N}_{2}\right]^{+}: 252.1388$, found: 252.1393 .

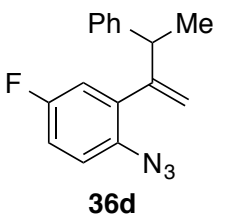

Styryl azide 36d. The general procedure was followed by using $0.130 \mathrm{~g}$ of $\mathbf{s 1 5 g}(0.540 \mathrm{mmol}), 0.28 \mathrm{~mL}$ of $t$-BuONO $(2.15 \mathrm{mmol}), 0.23 \mathrm{~mL}$ of $\mathrm{Me}_{3} \mathrm{SiN}_{3}(1.6 \mathrm{mmol})$ and $10 \mathrm{~mL}$ of MeCN. Purification by MPLC (2:98 - 10:90 EtOAc:hexanes) afforded the product as a colorless solid $(0.098 \mathrm{~g}, 68 \%):{ }^{1} \mathrm{H}$ NMR $\left(500 \mathrm{MHz}, \mathrm{CDCl}_{3}\right) \delta 7.29(\mathrm{t}, J=7.5$ $\mathrm{Hz}, 2 \mathrm{H}), 7.22(\mathrm{t}, J=8.2 \mathrm{~Hz}, 3 \mathrm{H}), 7.05(\mathrm{dd}, J=8.8 \mathrm{~Hz}, 4.7 \mathrm{~Hz}, 1 \mathrm{H}), 6.95(\mathrm{td}, J=8.3 \mathrm{~Hz}, 2.9 \mathrm{~Hz}, 1 \mathrm{H}), 6.69(\mathrm{dd}, J=9.0 \mathrm{~Hz}$, $2.9 \mathrm{~Hz}, 1 \mathrm{H}), 5.28(\mathrm{~s}, 1 \mathrm{H}), 5.13(\mathrm{~s}, 1 \mathrm{H}), 4.01(\mathrm{q}, J=7.3 \mathrm{~Hz}, 1 \mathrm{H}), 1.49(\mathrm{~d}, J=7.1 \mathrm{~Hz}, 3 \mathrm{H}) ;{ }^{13} \mathrm{C} \mathrm{NMR}\left(125 \mathrm{MHz}, \mathrm{CDCl}_{3}\right) \delta$ $159.2\left(\mathrm{C}, J_{C F}=245.8 \mathrm{~Hz}\right), 150.8(\mathrm{C}), 143.9(\mathrm{C}), 137.0(\mathrm{C}), 132.9(\mathrm{C}), 128.3(\mathrm{CH}), 127.8(\mathrm{CH}), 126.4(\mathrm{CH}), 119.5(\mathrm{CH}$, $\left.J_{C F}=8.8 \mathrm{~Hz}\right), 117.7\left(\mathrm{CH}, J_{C F}=22.1 \mathrm{~Hz}\right), 115.5\left(\mathrm{CH}_{2}\right), 115.0\left(\mathrm{CH}, J_{C F}=22.1 \mathrm{~Hz}\right), 45.0(\mathrm{CH}), 20.4\left(\mathrm{CH}_{3}\right) ;{ }^{19} \mathrm{~F} \mathrm{NMR}(282$ $\mathrm{MHz}, \mathrm{CDCl}_{3}$ ) $\delta-118.8$. ATR-FTIR (thin film): 3027, 2969, 2118, 2078, 1484, $1270 \mathrm{~cm}^{-1}$. HRMS (EI) $\mathrm{m} / z$ calculated for $\mathrm{C}_{16} \mathrm{H}_{14} \mathrm{~N}_{3} \mathrm{~F}[\mathrm{M}]^{+}: 267.1172$, found: 267.1185 . 


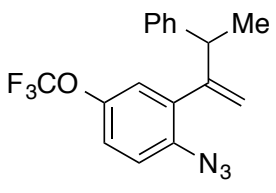

$36 f$

Styryl azide 36f. The general procedure was followed by using $0.160 \mathrm{~g}$ of $\mathbf{s 1 5 g}(0.520 \mathrm{mmol}), 0.27 \mathrm{~mL}$ of $t$-BuONO $(2.1$ $\mathrm{mmol}), 0.22 \mathrm{~mL}$ of $\mathrm{Me}_{3} \mathrm{SiN}_{3}$ (1.6 mmol) and $10 \mathrm{~mL}$ of MeCN. Purification by MPLC (2:98 - 10:90 EtOAc: hexanes) afforded the product as a colorless oil $(0.170 \mathrm{~g}, 98 \%)$ : ${ }^{1} \mathrm{H}$ NMR $\left(500 \mathrm{MHz}, \mathrm{CDCl}_{3}\right) \delta 7.27(\mathrm{t}, J=7.6 \mathrm{~Hz}, 2 \mathrm{H}), 7.19(\mathrm{~d}, J=$ $7.2 \mathrm{~Hz}, 3 \mathrm{H}), 7.10(\mathrm{~s}, 2 \mathrm{H}), 6.79(\mathrm{~s}, 1 \mathrm{H}), 5.30(\mathrm{~s}, 1 \mathrm{H}), 5.12(\mathrm{~s}, 1 \mathrm{H}), 3.97(\mathrm{q}, J=7.0 \mathrm{~Hz}, 1 \mathrm{H}), 1.49(\mathrm{~d}, J=7.1 \mathrm{~Hz}, 3 \mathrm{H}) ;{ }^{13} \mathrm{C}$ NMR (125 MHz, $\left.\mathrm{CDCl}_{3}\right) \delta 150.4(\mathrm{C}), 145.4(\mathrm{C}), 143.7(\mathrm{C}), 136.7(\mathrm{C}), 135.8(\mathrm{C}), 128.3(\mathrm{CH}), 127.8(\mathrm{CH}), 126.4(\mathrm{CH})$, $123.7(\mathrm{CH}), 120.9(\mathrm{CH}), 120.4\left(\mathrm{CF}_{3}, J_{C F}=257.1 \mathrm{~Hz}\right), 119.3(\mathrm{CH}), 115.6\left(\mathrm{CH}_{2}\right), 45.1(\mathrm{CH}), 20.1\left(\mathrm{CH}_{3}\right) ;{ }^{19} \mathrm{~F} \mathrm{NMR}(282$ $\left.\mathrm{MHz}, \mathrm{CDCl}_{3}\right) \delta-58.7$. ATR-FTIR (thin film): 2971, 2121, 2085, 1484, 1249, $1216 \mathrm{~cm}^{-1}$. HRMS (ES) $\mathrm{m} / z$ calculated for $\mathrm{C}_{17} \mathrm{H}_{15} \mathrm{NF}_{3} \mathrm{O}\left[\mathrm{M}+\mathrm{H}-\mathrm{N}_{2}\right]^{+}: 306.1106$, found: 306.1116 .

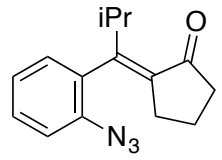

12

Styryl azide 12. The general procedure was followed by using $0.633 \mathrm{~g}$ of amine $\mathbf{s} 3 \mathbf{b}(2.76 \mathrm{mmol}), 1.31 \mathrm{~mL}$ of $t$-BuONO $(11.0 \mathrm{mmol}), 1.08 \mathrm{~mL}$ of $\mathrm{Me}_{3} \mathrm{SiN}_{3}(1.6 \mathrm{mmol})$ and $10 \mathrm{~mL}$ of MeCN. Purification by MPLC (3:97 to 10:90 EtOAc: hexane) afforded the product as colorless oil $(0.659 \mathrm{~g}, 93 \%)$ as a 79:21 mixture of $E$ - and $Z$-isomers. Characterization data for the major product: ${ }^{1} \mathrm{H}$ NMR $\left(500 \mathrm{MHz}, \mathrm{CDCl}_{3}\right) \delta 7.34(\mathrm{td}, J=7.7 \mathrm{~Hz}, 1.2 \mathrm{~Hz}, 1 \mathrm{H}), 7.20(\mathrm{~d}, J=7.9 \mathrm{~Hz}, 1 \mathrm{H}), 7.14(\mathrm{t}, J$ $=7.5 \mathrm{~Hz}, 1 \mathrm{H}), 6.96(\mathrm{dd}, J=7.6 \mathrm{~Hz}, 1.3 \mathrm{~Hz}, 1 \mathrm{H}), 4.34(\mathrm{hept}, J=6.9 \mathrm{~Hz}, 1 \mathrm{H}), 2.45-2.33(\mathrm{~m}, 2 \mathrm{H}), 2.26-2.21(\mathrm{~m}, 1 \mathrm{H})$, $2.12-2.07(\mathrm{~m}, 1 \mathrm{H}), 1.84-1.74(\mathrm{~m}, 1 \mathrm{H}), 1.74-1.62(\mathrm{~m}, 1 \mathrm{H}), 1.02(\mathrm{~d}, J=7.0 \mathrm{~Hz}, 3 \mathrm{H}), 0.85(\mathrm{~d}, J=6.5 \mathrm{~Hz}, 3 \mathrm{H}) ;{ }^{13} \mathrm{C}$ NMR (125 MHz, $\left.\mathrm{CDCl}_{3}\right) \delta 208.0(\mathrm{C}), 154.7(\mathrm{C}), 136.9(\mathrm{C}), 133.0(\mathrm{C}), 131.2(\mathrm{C}), 128.9(\mathrm{CH}), 128.6(\mathrm{CH}), 124.6(\mathrm{CH})$, $118.4(\mathrm{CH}), 41.1\left(\mathrm{CH}_{2}\right), 30.9\left(\mathrm{CH}_{2}\right), 28.5(\mathrm{CH}), 21.8\left(\mathrm{CH}_{3}\right), 20.3\left(\mathrm{CH}_{3}\right), 19.6\left(\mathrm{CH}_{2}\right)$; ATR-FTIR (thin film): 2961, 2871, 2116, 2084, 1704, 1616, $1287 \mathrm{~cm}^{-1}$. HRMS (ES) $\mathrm{m} / z$ calculated for $\mathrm{C}_{15} \mathrm{H}_{18} \mathrm{NO}\left[\mathrm{M}+\mathrm{H}-\mathrm{N}_{2}\right]^{+}:$228.1388, found: 228.1395 .

\section{Preparation of D-Labeled Substrates:}

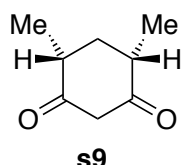

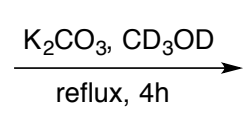

s9

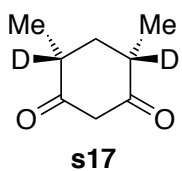

$(\mathrm{s} 21)$

4,6-Dimethylcyclohexane-1,3-dione-4,6- $\boldsymbol{d}_{2} \mathbf{s 1 7}$. To a flame dried conical flask, $0.500 \mathrm{~g}$ of cis-4,6-dimethylcyclohexane1,3 -dione $\mathbf{s 9}(3.56 \mathrm{mmol})$ in $10 \mathrm{~mL}$ methanol- $d_{4}$, was added $2.95 \mathrm{~g}$ of $\mathrm{K}_{2} \mathrm{CO}_{3}(21.4 \mathrm{mmol})$ and the mixture was refluxed at $90{ }^{\circ} \mathrm{C}$. After 4 hours, the reaction mixture was then cooled to room temperature, and the reactives were quenched through the addition of $2.0 \mathrm{~mL}$ of acetic acid- $d_{4}(34.9 \mathrm{mmol})$. The mixture was then refluxed to reflux. After 2 hours, the solution was cooled to room temperature, and $10 \mathrm{~mL}$ of water was added to the reaction mixture. The resulting mixture was extracted with $2 \times 20 \mathrm{~mL}$ of ethyl acetate, and the combined organic layers was extracted with $10 \mathrm{~mL}$ of brine. The resulting organic phase was dried over $\mathrm{Na}_{2} \mathrm{SO}_{4}$, filtered, and the filtrate was concentrated in vacuo. Purification by MPLC (2:98 to 50:50 EtOAc:hexanes) afforded the cis-isomer as a white solid $(0.312 \mathrm{~g}, 61 \%):{ }^{1} \mathrm{H}$ NMR $\left(500 \mathrm{MHz}, \mathrm{CDCl}_{3}\right) \delta$ 3.46 and $3.38(\mathrm{ABq}, J=16.0 \mathrm{~Hz}, 2 \mathrm{H}), 2.68(\mathrm{~m}, 0.27 \mathrm{H}), 2.12(\mathrm{~d}, J=13.5 \mathrm{~Hz}, 1 \mathrm{H}), 1.44(\mathrm{~m}, 0.56 \mathrm{H}), 1.14(\mathrm{~s}, 6 \mathrm{H})$, only peaks visible; ATR-FTIR (thin film): 2964, 2931, 2832, 2574, 1731, 1708, 1595, 1456, 1375, 1312, 1257, $1195 \mathrm{~cm}^{-1}$. 


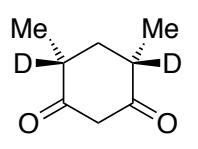

s17

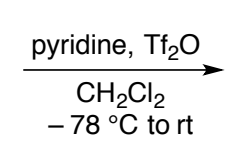

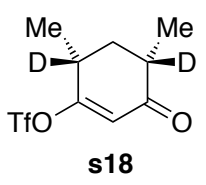

(s22)

Vinyl triflate s18. To a $-78{ }^{\circ} \mathrm{C}$ solution of $0.320 \mathrm{~g}$ of 4,6-dimethylcyclohexane-1,3-dione-4,6- $d_{2} \mathbf{s 1 7}(2.25 \mathrm{mmol})$ and $0.360 \mathrm{~mL}$ of pyridine $(4.50 \mathrm{mmol})$ in dichloromethane $(20 \mathrm{~mL})$ at $-78{ }^{\circ} \mathrm{C}$ was added $0.45 \mathrm{~mL}$ of trifluoromethanesulfonic anhydride $(2.70 \mathrm{mmol})$ slowly. The reaction mixture was stirred for $10 \mathrm{~min}$ at $-78{ }^{\circ} \mathrm{C}$ followed by warming it to room temperature. After 4 hours, the reaction mixture was then quenched with $3.0 \mathrm{~mL}$ of a $1 \mathrm{M}$ aq. solution of $\mathrm{HCl}$. The resulting mixture was extracted with $3 \times 10 \mathrm{~mL}$ of diethyl ether. The combined organic layer was washed with $10 \mathrm{~mL}$ of a saturated aq. solution of $\mathrm{NaHCO}_{3}$ solution, followed by $10 \mathrm{~mL}$ of brine. The resulting organic phase was dried over $\mathrm{Na}_{2} \mathrm{SO}_{4}$, filtered, and the filtrate was concentrated in vacuo to produce the crude product as a brown oil $(0.477 \mathrm{~g}, 77 \%)$. This crude product was used in the next step without further purification. ATR-FTIR (thin film): 2975, 2938, 1692, 1639, $1459,1422,1246,1207,1135,1021 \mathrm{~cm}^{-1}$.

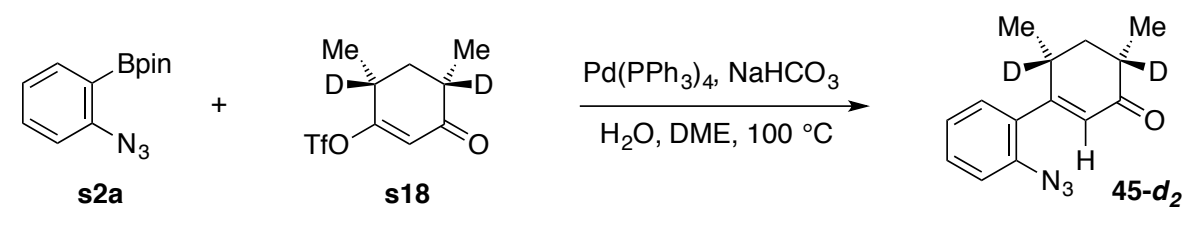

Azide $45-\boldsymbol{d}_{2}$. To a mixture of $0.115 \mathrm{~g}$ of vinyl triflate $\mathbf{s 1 8}(0.420 \mathrm{mmol}), 0.113 \mathrm{~g}$ of 2-azidoarylboronic acid pinacol ester s2a $(0.460 \mathrm{mmol})$, and $0.029 \mathrm{~g}$ of $\mathrm{PdCl}_{2}\left(\mathrm{PPh}_{3}\right)_{2}(10 \mathrm{~mol} \%)$ in $4.6 \mathrm{~mL}$ of dimethoxyethane $(0.1 \mathrm{M})$ was added a $0.90 \mathrm{~mL}$ saturated aq. solution of $\mathrm{NaHCO}_{3}\left(2 \mathrm{~mL} / \mathrm{mmol}\right.$ of boronic ester). The resulting mixture was heated to $100{ }^{\circ} \mathrm{C}$. After $1 \mathrm{~h}$, the mixture was cooled to room temperature and diluted with $5 \mathrm{~mL}$ of cold water. The solution was extracted with $2 \times 10$ $\mathrm{mL}$ of ether followed by $10 \mathrm{~mL}$ of brine. The resulting organic phase was dried over $\mathrm{Na}_{2} \mathrm{SO}_{4}$, filtered and the filtrate was concentrated in vacuo. Purification by MPLC (3:97 to 10:90 EtOAc: hexanes) afforded the cis-isomer (0.058 g, 57\%) as a yellow oil, $81 \% \mathrm{D}$ was exchanged in the allylic position: ${ }^{1} \mathrm{H} \mathrm{NMR}\left(500 \mathrm{MHz}, \mathrm{CDCl}_{3}\right) \delta 7.37(\mathrm{t}, J=7.0 \mathrm{~Hz}, 1 \mathrm{H}), 7.18-$ $7.09(\mathrm{~m}, 3 \mathrm{H}), 5.93(\mathrm{~s}, 1 \mathrm{H}), 3.28(\mathrm{~m}, 0.19 \mathrm{H}), 2.55(\mathrm{~m}, 0.21 \mathrm{H}), 2.14(\mathrm{~d}, J=13.0 \mathrm{~Hz}, 1 \mathrm{H}), 1.52(\mathrm{~d}, J=12.5 \mathrm{~Hz}, 1 \mathrm{H}), 1.16$ (s, 3H), $0.86(\mathrm{~s}, 3 \mathrm{H}) ;{ }^{13} \mathrm{C}$ NMR (125 MHz, $\left.\mathrm{CDCl}_{3}\right) \delta 201.9(\mathrm{C}), 164.1(\mathrm{C}), 136.5(\mathrm{C}), 131.8(\mathrm{C}), 129.5(\mathrm{CH}), 129.4(\mathrm{CH})$, $128.9(\mathrm{CH}), 125.0(\mathrm{CH}), 118.2(\mathrm{CH}), 41.4(\mathrm{CD}), 40.7\left(\mathrm{CH}_{2}\right), 34.1(\mathrm{CD}), 19.9\left(\mathrm{CH}_{3}\right), 14.8\left(\mathrm{CH}_{3}\right)$ only peaks visible (allylic methine $\mathrm{CH}$ signals present from the 19\% unlabeled azide); ATR-FTIR (thin film): 2959, 2927, 2870, 2122, 1672, 1609, $1484,1441,1286,1154 \mathrm{~cm}^{-1}$. HRMS (ES) $\mathrm{m} / z$ calculated for $\mathrm{C}_{14} \mathrm{H}_{14} \mathrm{D}_{2} \mathrm{NO}\left[\mathrm{M}+\mathrm{H}-\mathrm{N}_{2}\right]^{+}: 216.1357$, found: 216.1362 .
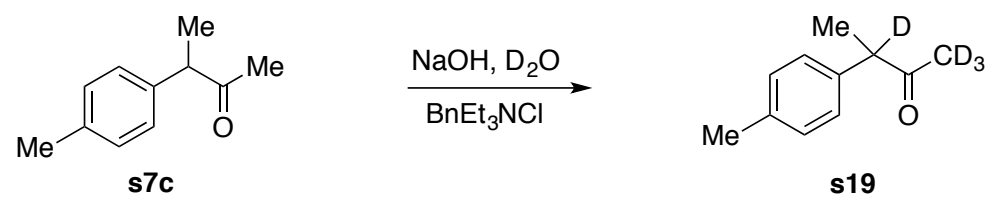

3-para-Methylphenyl-2-butanone- $\boldsymbol{d}_{\mathbf{2}} \mathbf{s 1 9}$. A mixture of $0.200 \mathrm{~g}$ of 3-para-methylphenyl-2-butanone $\mathbf{s} 7 \mathrm{c}(1.35 \mathrm{mmol})$, $16 \mathrm{~mL}$ of a $50 \% \mathrm{NaOH}$ aqueous solution in $\mathrm{D}_{2} \mathrm{O}(8.09 \mathrm{mmol})$, and $0.018 \mathrm{~g}$ benzyltrimethylammonium chloride $(6 \mathrm{~mol} \%)$ was stirred vigorously using a mechanical stirrer. The temperature of the solution was kept around room temperature by an ice bath. After stirring for $1 \mathrm{~h}, 20 \mathrm{~mL}$ of $\mathrm{H}_{2} \mathrm{O}$ and $30 \mathrm{~mL}$ of ethyl acetate were added to the reaction mixture. The organic layer was separated, and was washed with $\mathrm{H}_{2} \mathrm{O}$ until its $\mathrm{pH}$ became neutral. The resulting organic phase was extracted with $10 \mathrm{~mL}$ of brine. The organic layer was separated and dried over $\mathrm{Na}_{2} \mathrm{SO}_{4}$, filtered, and the filtrate was concentrated in vacuo to produce a residue $(0.196 \mathrm{~g}, 97 \%)$, which was submitted to the next step without additional purification. ${ }^{1} \mathrm{H}$ NMR $\left(500 \mathrm{MHz}, \mathrm{CDCl}_{3}\right) \delta 7.14(\mathrm{~d}, J=8.0 \mathrm{~Hz}, 2 \mathrm{H}), 7.11(\mathrm{~d}, J=8.0 \mathrm{~Hz}, 2 \mathrm{H}), 2.33(\mathrm{~s}, 3 \mathrm{H}), 1.36(\mathrm{~s}, 3 \mathrm{H})$; ATR-FTIR (thin film): 2975, 2930, 1708, 1513, 1451, 1372, 1266, 1243, 1126, $1007 \mathrm{~cm}^{-1}$. 

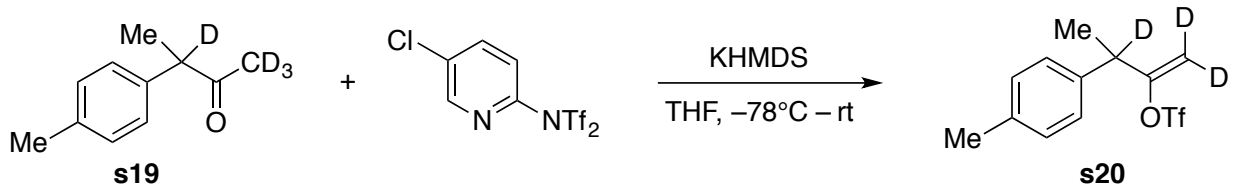

(s25)

Vinyl triflate s20. To a $-78{ }^{\circ} \mathrm{C}$ solution of $0.196 \mathrm{~g}$ of KHMDS $(0.980 \mathrm{mmol})$ in $5.0 \mathrm{~mL}$ of THF $(0.2 \mathrm{M})$ was slowly added a solution of $0.125 \mathrm{~g}$ of 3-p-methylphenyl-2-butanone- $d_{2} \mathbf{s 1 9}(0.800 \mathrm{mmol})$ in THF. After stirring for $0.5 \mathrm{~h}$, a solution of $0.354 \mathrm{~g}$ of Comins' reagent $(0.900 \mathrm{mmol})$ in THF was added dropwise to the reaction. The resulting mixture was then gradually warmed to room temperature. After stirring for overnight, the reaction was quenched by the addition of a saturated aq. solution of $\mathrm{NH}_{4} \mathrm{Cl}$. The mixture was then extracted with EtOAc. The combined organic phases were washed with brine. The resulting organic phase was dried over $\mathrm{Na}_{2} \mathrm{SO}_{4}$, filtered and the filtrate was concentrated in vacuo. Purification by MPLC (2:98 to 5:95 EtOAc: hexane) afforded the product as a colorless oil $(0.095 \mathrm{~g}, 39 \%)$ : ${ }^{1} \mathrm{H}$ NMR (500 MHz, $\left.\mathrm{CDCl}_{3}\right) \delta 7.12(\mathrm{~m}, 4 \mathrm{H}), 2.34$ (s, 3H), 1.46 (s, 3H); ATR-FTIR (thin film): 2980, 1622, 1514, 1415, $1248,1140,1024 \mathrm{~cm}^{-1}$.
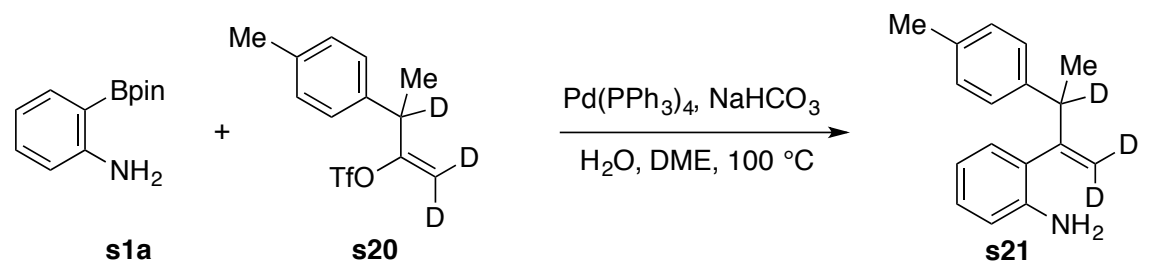

Aniline s21: To a mixture of $0.190 \mathrm{~g}$ of vinyl triflate $\mathbf{s 2 0}(0.640 \mathrm{mmol}), 0.128 \mathrm{~g}$ of 2 -aminoarylboronic acid pinacol ester s1a $(0.580 \mathrm{mmol})$, and $0.033 \mathrm{~g}$ of $\mathrm{Pd}\left(\mathrm{PPh}_{3}\right)_{4}(5 \mathrm{~mol} \%)$ in $5.8 \mathrm{~mL}$ of dimethoxyethane $(0.1 \mathrm{M})$ was added $1.2 \mathrm{~mL}$ of a saturated aq. solution of $\mathrm{NaHCO}_{3}(2 \mathrm{~mL} / \mathrm{mmol}$ of boronic ester $)$. The resulting mixture was heated to $100{ }^{\circ} \mathrm{C}$. After $4 \mathrm{~h}$, the mixture was cooled to room temperature and diluted with $5 \mathrm{~mL}$ of cold water. The solution was extracted with $2 \times 10$ $\mathrm{mL}$ of diethyl ether followed by $10 \mathrm{~mL}$ of brine. The phases were separated, and the resulting organic phase was dried over $\mathrm{Na}_{2} \mathrm{SO}_{4}$, filtered and the filtrate was concentrated in vacuo. Purification by MPLC (3:97 to 50:50 EtOAc:hexanes) afforded the product $\mathbf{s 2 1}$ as a colorless oil $(0.134 \mathrm{~g}, 96 \%):{ }^{1} \mathrm{H} \mathrm{NMR}\left(500 \mathrm{MHz}, \mathrm{CDCl}_{3}\right) \delta 7.09(\mathrm{AB} \mathrm{q}, J=8.0 \mathrm{~Hz}, 4 \mathrm{H})$, $7.03(\mathrm{dt}, J=8.0 \mathrm{~Hz}, 1.5 \mathrm{~Hz}, 1 \mathrm{H}), 6.82(\mathrm{dd}, J=7.5 \mathrm{~Hz}, 1.0 \mathrm{~Hz}, 1 \mathrm{H}), 6.65(\mathrm{~m}, 2 \mathrm{H}), 3.61(\mathrm{~s}, 2 \mathrm{H}), 2.32(\mathrm{~s}, 3 \mathrm{H}), 1.45(\mathrm{~d}, 3 \mathrm{H})$; ${ }^{13} \mathrm{C}$ NMR $\left(125 \mathrm{MHz}, \mathrm{CDCl}_{3}\right) \delta 151.8(\mathrm{C}), 143.3(\mathrm{C}), 141.2(\mathrm{C}), 135.7(\mathrm{C}), 129.0(\mathrm{CH}), 128.9(\mathrm{CH}), 128.8(\mathrm{C}), 127.8$ $(\mathrm{CH}), 127.6(\mathrm{CH}), 117.8(\mathrm{CH}), 115.4(\mathrm{CH}), 45.2\left(\mathrm{t}, J_{C D}=19.2 \mathrm{~Hz}, \mathrm{CD}\right), 21.1\left(\mathrm{CH}_{3}\right), 20.2\left(\mathrm{CH}_{3}\right)$, only peaks visible; ATRFTIR (thin film): 3459, 3372, 3018, 2965, 2927, 2869, 1611, 1512, 1490, 1447, $1295 \mathrm{~cm}^{-1}$.
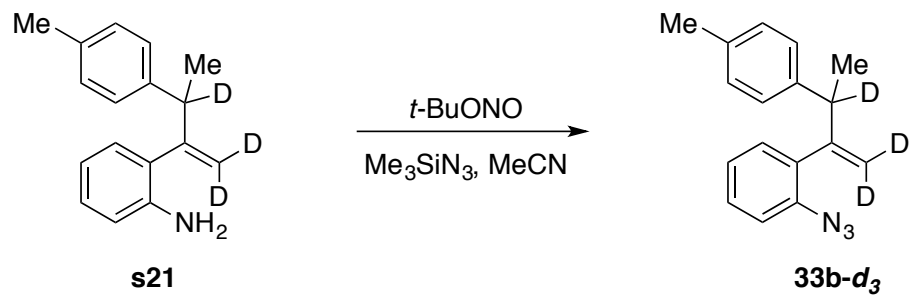

(s27)

Azide 33b- $\boldsymbol{d}_{3}$. To a cooled solution of $0.053 \mathrm{~g}$ of aniline $\mathbf{s 2 1}(0.22 \mathrm{mmol})$ in $2.2 \mathrm{~mL}$ of MeCN was added dropwise 0.10 $\mathrm{mL}$ of $t$-BuONO $(0.90 \mathrm{mmol})$ and $0.087 \mathrm{~mL}$ of $\mathrm{Me}_{3} \mathrm{SiN}_{3}(0.66 \mathrm{mmol})$. The resulting solution was warmed to room temperature. After $1 \mathrm{~h}$, visualization of the reaction progress using TLC indicated the consumption of the starting material. De-ionized water was added to the reaction mixture. The mixture then was extracted with $2 \times 15 \mathrm{~mL}$ of $\mathrm{CH}_{2} \mathrm{Cl}_{2}$. The combined organic phases were washed with $10 \mathrm{~mL}$ of brine. The resulting organic phase was dried over $\mathrm{Na}_{2} \mathrm{SO}_{4}$, filtered, and the filtrate was concentrated in vacuo. Purification of the residue by MPLC (2:98 - 10:90 EtOAc:hexanes) afforded azide as a colorless oil $(0.053 \mathrm{~g}, 88 \%)$ : ${ }^{1} \mathrm{H}$ NMR $\left(500 \mathrm{MHz}, \mathrm{CDCl}_{3}\right) \delta 7.24(\mathrm{~m}, 1 \mathrm{H}), 7.11-7.04$ $(\mathrm{m}, 5 \mathrm{H}), 6.97(\mathrm{t}, J=7.5 \mathrm{~Hz}, 1 \mathrm{H}), 6.93(\mathrm{~m}, 1 \mathrm{H}), 2.29(\mathrm{~s}, 3 \mathrm{H}), 1.41(\mathrm{~s}, 3 \mathrm{H}) ;{ }^{13} \mathrm{C} \mathrm{NMR}\left(125 \mathrm{MHz}, \mathrm{CDCl}_{3}\right) \delta 151.7(\mathrm{C}), 141.2$ $(\mathrm{C}), 136.9(\mathrm{C}), 135.6(\mathrm{C}), 135.3(\mathrm{C}), 130.9(\mathrm{CH}), 128.9(\mathrm{CH}), 128.2(\mathrm{CH}), 127.7(\mathrm{CH}), 124.4(\mathrm{CH}), 118.2(\mathrm{CH}), 21.0$ 
$\left(\mathrm{CH}_{3}\right), 20.4\left(\mathrm{CH}_{3}\right)$, only peaks visible; ATR-FTIR (thin film): 3019, 2966, 2927, 2870, 2122, 2088, 1585, 1572, 1512, $1481,1439,1287 \mathrm{~cm}^{-1}$. HRMS (EI) $\mathrm{m} / z$ calculated for $\mathrm{C}_{17} \mathrm{H}_{15} \mathrm{D}_{3} \mathrm{~N}\left[\mathrm{M}+\mathrm{H}-\mathrm{N}_{2}\right]^{+}: 239.1628$, found: 239.1625 .

\section{Investigation of Catalyst Control of Site Selectivity.}

A. Conditions Examined for Styryl Azide 35c.<smiles>C=C(c1ccccc1N(C)C)C(C)c1ccccc1</smiles>

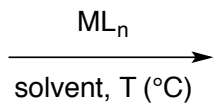

$36 c$

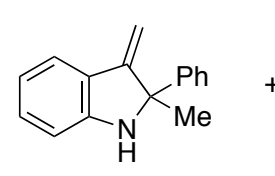

$37 c$<smiles>CC(C)c1c[nH]c2ccccc12</smiles>

To a mixture of styryl azide 36c and a metal catalyst $(5 \mathrm{~mol} \%)$ was added toluene $(0.1 \mathrm{M})$. The resulting mixture was heated. After $16 \mathrm{~h}$, the mixture was cooled to room temperature, diluted with $\mathrm{CH}_{2} \mathrm{Cl}_{2}$ and concentrated in vacuo. Dibromomethane $(0.7 \mu \mathrm{L}, 0.1 \mathrm{mmol})$ was added, and the mixture was analyzed using ${ }^{1} \mathrm{H}$ NMR spectroscopy to determine the outcome of the reaction.

Table s1. Conditions for the screening styryl azide $36 \mathrm{c}$ with different catalysts.

\begin{tabular}{ccccc}
\hline entry & $\mathrm{ML}_{\mathrm{n}}$ & $\mathrm{T}\left({ }^{\circ} \mathrm{C}\right)$ & $\mathbf{3 7 : 3 8}$ & $\%$, yield $^{\mathrm{a}}$ \\
\hline $1^{\mathrm{b}}$ & none & 220 & $2: 98$ & 77 \\
2 & $\mathrm{Rh}_{2}(\mathrm{esp})_{2}$ & 140 & $14: 86$ & 93 \\
3 & $\mathrm{Rh}_{2}(\mathrm{esp})_{2}$ & 120 & $13: 87$ & 95 \\
4 & $\mathrm{Rh}_{2}(\mathrm{esp})_{2}$ & 100 & $12: 88$ & 88 \\
5 & $\mathrm{Rh}_{2}\left(\mathrm{O}_{2} \mathrm{CCF}_{3}\right)_{4}$ & 120 & $2: 98$ & 66 \\
6 & $\mathrm{Rh}_{2}\left(\mathrm{O}_{2} \mathrm{CC}_{3} \mathrm{~F}_{7}\right)_{4}$ & 120 & $6: 84$ & $50^{\mathrm{c}}$ \\
7 & $\mathrm{Rh}_{2}\left(\mathrm{O}_{2} \mathrm{CC}_{7} \mathrm{H}_{15}\right)_{4}$ & 120 & $11: 89$ & 93 \\
8 & $\mathrm{Rh}_{2}(\mathrm{~S}-\mathrm{DOSP})_{4}$ & 120 & $18: 82$ & 79 \\
9 & $\mathrm{Rh}_{2}(\mathrm{cap})_{4}$ & 120 & $4: 96$ & 82 \\
10 & {$[\mathrm{Ir}(\mathrm{COD})(\mathrm{OMe})]_{2}$} & 120 & $0: 100$ & 84 \\
11 & $\mathrm{RuCl}_{3}$ & 120 & - & 0 \\
\hline Yield determined by ${ }^{1} \mathrm{H} \mathrm{NMR} \mathrm{spectroscopy} \mathrm{using} \mathrm{CH}_{2} \mathrm{Br}_{2}$ as an internal standard. \\
Mesitylene was used as solvent. ${ }^{\mathrm{c}} 81 \%$ conversion of starting material was observed.
\end{tabular}

\section{B. Condition Examined for Styryl Azide 44.}<smiles>[M]C1CC(C)C(=O)C=C1c1ccccc1N</smiles>

45

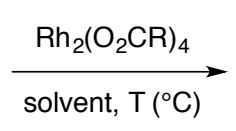

solvent, $\mathrm{T}\left({ }^{\circ} \mathrm{C}\right)$

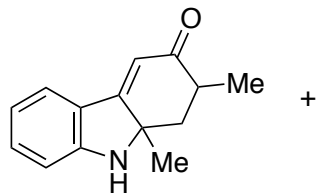

46<smiles>CC1CC(C)c2c([nH]c3ccccc23)C1=O</smiles>

47

To a mixture of styryl azide $\mathbf{4 5}$ and a metal catalyst was added solvent $(0.1 \mathrm{M})$ and $100 \mathrm{wt} \%$ of $4 \AA$ MS. The resulting mixture was heated. After $16 \mathrm{~h}$, the mixture was cooled to room temperature, diluted with $\mathrm{CH}_{2} \mathrm{Cl}_{2}$ and concentrated in vacuo. Dibromomethane $(0.7 \mu \mathrm{L}, 0.1 \mathrm{mmol})$ was added, and the mixture was analyzed using ${ }^{1} \mathrm{H}$ NMR spectroscopy to determine the outcome of the reaction. 
Table s2. Conditions examined for styryl azide 44.

\begin{tabular}{|c|c|c|c|c|c|c|}
\hline entry & $M L_{n}$ & $\mathrm{~mol} \%$ & $\mathrm{~T}\left({ }^{\circ} \mathrm{C}\right)$ & solvent & $46: 47$ & $\%$, yield ${ }^{a}$ \\
\hline 1 & none & - & 125 & PhMe & $94: 6$ & 15 \\
\hline 2 & $\mathrm{Rh}_{2}(\mathrm{esp})_{2}$ & 5 & 120 & PhMe & $62: 38$ & 91 \\
\hline 3 & $\mathrm{Rh}_{2}(\mathrm{esp})_{2}$ & 5 & 110 & PhMe & 63:37 & 90 \\
\hline 4 & $\mathrm{Rh}_{2}\left(\mathrm{O}_{2} \mathrm{CC}_{7} \mathrm{H}_{15}\right)_{4}$ & 5 & 110 & PhMe & $68: 32$ & 78 \\
\hline 5 & $\mathrm{Rh}_{2}(S-\mathrm{DOSP})_{4}$ & 5 & 100 & PhMe & $44: 56$ & $64^{c}$ \\
\hline 6 & $\mathrm{Rh}_{2}(\mathrm{cap})_{4}$ & 5 & 100 & PhMe & $91: 9$ & 73 \\
\hline 7 & $\mathrm{Rh}_{2}(S-\mathrm{PTAD})_{4}$ & 5 & 110 & PhMe & $20: 80$ & 84 \\
\hline 8 & $\mathrm{Rh}_{2}(S-\mathrm{PTAD})_{4}$ & 5 & 100 & PhMe & 18:82 & 85 \\
\hline 9 & $\mathrm{Rh}_{2}(S-\mathrm{PTAD})_{4}$ & 2 & 100 & PhMe & $26: 74$ & $57^{d}$ \\
\hline 10 & $\mathrm{Rh}_{2}(S-\mathrm{PTAD})_{4}$ & 5 & 90 & PhMe & $17: 83$ & $61^{\mathrm{e}}$ \\
\hline 11 & $\mathrm{Rh}_{2}(S-\mathrm{PTAD})_{4}$ & 2 & 110 & $\mathrm{PhMe}+\mathrm{BzEt}_{3} \mathrm{NCl}$ & $26: 74$ & 74 \\
\hline 12 & $\mathrm{Rh}_{2}(S-\mathrm{PTAD})_{4}$ & 2 & 110 & $\mathrm{PhMe}^{\mathrm{b}}+\mathrm{BzEt}_{3} \mathrm{NCl}$ & $20: 80$ & 72.5 \\
\hline 13 & $\mathrm{Rh}_{2}(S-\mathrm{PTAD})_{4}$ & 5 & 100 & $\mathrm{PhMe}^{\mathrm{b}}+\mathrm{BzEt}_{3} \mathrm{NCl}$ & $17: 83$ & 81 \\
\hline 14 & $\mathrm{Rh}_{2}(S-\mathrm{PTAD})_{4}$ & 5 & 110 & DCE & 19:81 & 86 \\
\hline 15 & $\mathrm{Rh}_{2}(S-\mathrm{PTAD})_{4}$ & 5 & 110 & DMSO- $d_{6}$ & $100: 0$ & $0^{\dagger}$ \\
\hline 16 & {$[\operatorname{Ir}(\mathrm{cod}) \mathrm{OMe}]_{2}$} & 5 & 110 & PhMe & 100:0 & 88 \\
\hline 17 & $\mathrm{FeBr}_{2}$ & 20 & 110 & $\mathrm{PhMe}^{\mathrm{b}}$ & 100:0 & 84 \\
\hline 18 & $\mathrm{FeBr}_{3}$ & 20 & 110 & $\mathrm{PhMe}^{\mathrm{b}}$ & - & 41 \\
\hline 19 & CoTPP & 2 & 90 & $\mathrm{MeCN}^{\mathrm{b}}$ & $22: 78$ & 0 \\
\hline 20 & CoTPP & 2 & 100 & $\mathrm{DCE}^{\mathrm{b}}$ & 11:89 & 68 \\
\hline 21 & COOEP & 2 & 100 & $\mathrm{DCE}^{\mathrm{b}}$ & $6: 94$ & $18^{g}$ \\
\hline 22 & $\mathrm{Fe}(\mathrm{TPP}) \mathrm{Cl}$ & 2 & 90 & $\mathrm{DCE}^{\mathrm{b}}$ & $7: 93$ & 47 \\
\hline 23 & $\mathrm{Fe}(\mathrm{TPP}) \mathrm{Cl}$ & 2 & 100 & $\mathrm{DCE}^{\mathrm{b}}$ & $10: 90$ & 52 \\
\hline 24 & $\mathrm{Fe}(\mathrm{TPP}) \mathrm{Cl}$ & 2 & 110 & $\mathrm{DCE}^{\mathrm{b}}$ & $12: 88$ & 48.5 \\
\hline 25 & $\mathrm{Fe}(\mathrm{TPP}) \mathrm{Cl}$ & 2 & 125 & $\mathrm{DCE}^{\mathrm{b}}$ & $13: 87$ & 52 \\
\hline 26 & $\mathrm{Fe}(\mathrm{TPP}) \mathrm{Cl}$ & 2 & 100 & $\mathrm{DCE}^{\mathrm{b}}+\mathrm{AgBF}_{4}$ & - & 30 \\
\hline 27 & none & & 100 & $\mathrm{DCE}^{\mathrm{b}}+\mathrm{AgBF}_{4}$ & $23: 77$ & 0 \\
\hline 28 & $\mathrm{Fe}(\mathrm{TPFPP}) \mathrm{Cl}$ & 2 & 110 & $\mathrm{DCE}^{\mathrm{b}}$ & $6: 94$ & 72 \\
\hline 29 & $\mathrm{Fe}(\mathrm{TOMePP}) \mathrm{Cl}$ & 2 & 100 & $\mathrm{DCE}^{\mathrm{b}}$ & $2: 98$ & 52 \\
\hline 30 & $\mathrm{Fe}(\mathrm{OEP}) \mathrm{Cl}$ & 2 & 100 & $\mathrm{DCE}^{\mathrm{b}}$ & 100:0 & 77 \\
\hline
\end{tabular}

\section{Rh ${ }_{2}$ (II)-Catalyzed $\mathrm{sp}^{3} \mathrm{C}-\mathrm{H}$ Bond Amination and Electrocyclization.}

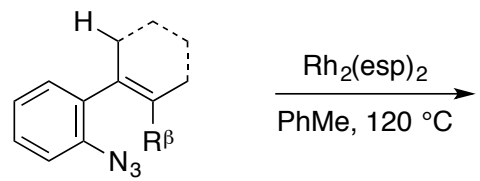<smiles>BrCCC1CCCc2[nH]c3ccccc3c21</smiles>

To a mixture of styryl azide and $\mathrm{Rh}_{2}(\mathrm{esp})_{2}(5 \mathrm{~mol} \%)$ was added toluene $(0.1 \mathrm{M})$. The resulting mixture was heated at 120 ${ }^{\circ} \mathrm{C}$. After $16 \mathrm{~h}$, the mixture was cooled to room temperature, diluted with $\mathrm{CH}_{2} \mathrm{Cl}_{2}$ and concentrated in vacuo. Purification of the residue by MPLC (3:97 - 30:70 EtOAc:hexanes) using silica gel afforded the product. 


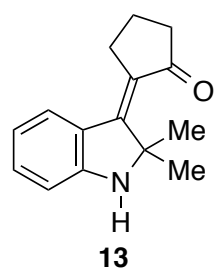

Alkylidene indoline 13. The general procedure was followed by using $0.0300 \mathrm{~g}$ of styryl azide $12(0.120 \mathrm{mmol}), 0.0045$ $\mathrm{g}$ of $\mathrm{Rh}_{2}(\mathrm{esp})_{2}(5 \mathrm{~mol} \%)$ and $1.2 \mathrm{~mL}$ of toluene. Purification by MPLC (3:97 to 10:90 EtOAc:hexanes) afforded the product as a yellow solid $(0.0222 \mathrm{~g}, 83 \%):{ }^{1} \mathrm{H} \mathrm{NMR}\left(500 \mathrm{MHz}, \mathrm{CDCl}_{3}\right) \delta 7.48(\mathrm{~d}, J=8.0 \mathrm{~Hz}, 1 \mathrm{H}), 7.20(\mathrm{t}, J=7.6 \mathrm{~Hz}$, $1 \mathrm{H}), 6.71(\mathrm{t}, J=7.6 \mathrm{~Hz}, 1 \mathrm{H}), 6.61(\mathrm{~d}, J=8.0 \mathrm{~Hz}, 1 \mathrm{H}), 4.17(\mathrm{~s}, 1 \mathrm{H}), 3.00(\mathrm{t}, J=7.2 \mathrm{~Hz}, 2 \mathrm{H}), 2.36(\mathrm{t}, J=7.9 \mathrm{~Hz}, 2 \mathrm{H}), 2.02$ (quintet, $J=7.6 \mathrm{~Hz}, 2 \mathrm{H}), 1.70(\mathrm{~s}, 6 \mathrm{H}) ;{ }^{13} \mathrm{C} \mathrm{NMR}\left(125 \mathrm{MHz}, \mathrm{CDCl}_{3}\right) \delta 206.8(\mathrm{C}), 156.6(\mathrm{C}), 154.7(\mathrm{C}), 132.6(\mathrm{CH}), 127.8$ $(\mathrm{CH}), 126.0(\mathrm{C}), 125.6(\mathrm{C}), 117.9(\mathrm{CH}), 109.7(\mathrm{CH}), 66.5(\mathrm{C}), 40.2\left(\mathrm{CH}_{2}\right), 33.1\left(\mathrm{CH}_{2}\right), 25.3\left(\mathrm{CH}_{3}\right), 20.3\left(\mathrm{CH}_{2}\right)$; ATRFTIR (thin film): $3335,2960,2853,1679,1557,1313 \mathrm{~cm}^{-1}$. HRMS (ES) $\mathrm{m} / z$ calculated for $\mathrm{C}_{15} \mathrm{H}_{18} \mathrm{NO}[\mathrm{M}+\mathrm{H}]^{+}$: 228.1388, found: 228.1394

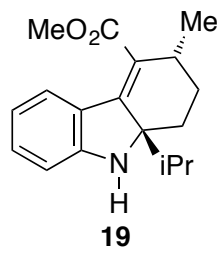

Alkylidene indoline 19. The general procedure was followed by using $0.0393 \mathrm{~g}$ of styryl azide $18(0.125 \mathrm{mmol}), 0.0047$ $\mathrm{g}$ of $\mathrm{Rh}_{2}(\mathrm{esp})_{2}(5 \mathrm{~mol} \%)$ and $1.3 \mathrm{~mL}$ of toluene. Purification by MPLC (3:97 to 10:90 EtOAc:hexanes) afforded the product, a white solid, as 91:9 mixture of diastereomers $(0.0268 \mathrm{~g}, 75 \%)$. The identity of the major diastereomer was determined by nOe: ${ }^{1} \mathrm{H}$ NMR $\left(500 \mathrm{MHz}, \mathrm{CDCl}_{3}\right) \delta 7.63(\mathrm{~d}, J=7.8 \mathrm{~Hz}, 1 \mathrm{H}), 7.09(\mathrm{t}, J=7.6 \mathrm{~Hz}, 1 \mathrm{H}), 6.66(\mathrm{t}, J=7.6 \mathrm{~Hz}$, $1 \mathrm{H}), 6.63(\mathrm{~d}, J=7.9 \mathrm{~Hz}, 1 \mathrm{H}), 4.18(\mathrm{~s}, 1 \mathrm{H}), 3.83(\mathrm{~s}, 3 \mathrm{H}), 2.99$ (hextet, $J=7.7 \mathrm{~Hz}, 1 \mathrm{H}), 2.15(\mathrm{dt}, J=12.7 \mathrm{~Hz}, 3.3 \mathrm{~Hz}, 1 \mathrm{H})$, $2.01(\mathrm{~m}, 1 \mathrm{H}), 1.89(\mathrm{dt}, J=13.7 \mathrm{~Hz}, 6.8 \mathrm{~Hz}, 1 \mathrm{H}), 1.53(\mathrm{td}, J=13.8 \mathrm{~Hz}, 4.3 \mathrm{~Hz}, 1 \mathrm{H}), 1.35$ (tdd, $J=14.1 \mathrm{~Hz}, 9.3 \mathrm{~Hz}, 4.6 \mathrm{~Hz}$, $1 \mathrm{H}), 1.13(\mathrm{~d}, J=7.0 \mathrm{~Hz}, 3 \mathrm{H}), 0.95(\mathrm{~d}, J=6.9 \mathrm{~Hz}, 3 \mathrm{H}), 0.68(\mathrm{~d}, J=6.8 \mathrm{~Hz}, 3 \mathrm{H}) ;{ }^{13} \mathrm{C} \mathrm{NMR}\left(125 \mathrm{MHz}, \mathrm{CDCl}_{3}\right) \delta 170.0(\mathrm{C})$, $153.2(\mathrm{C}), 148.1(\mathrm{C}), 130.5(\mathrm{CH}), 126.0(\mathrm{C}), 125.3(\mathrm{C}), 124.2(\mathrm{CH}), 118.5(\mathrm{CH}), 109.2(\mathrm{CH}), 67.1(\mathrm{C}), 51.5\left(\mathrm{CH}_{3}\right), 36.2$ $(\mathrm{CH}), 32.5\left(\mathrm{CH}_{2}\right), 32.1(\mathrm{CH}), 26.4\left(\mathrm{CH}_{2}\right), 22.7\left(\mathrm{CH}_{3}\right), 17.4\left(\mathrm{CH}_{3}\right), 16.6\left(\mathrm{CH}_{3}\right)$; ATR-FTIR (thin film): 3392, 2954,2870 , $1709,1603,1466,1248 \mathrm{~cm}^{-1}$. HRMS (ES) $\mathrm{m} / z$ calculated for $\mathrm{C}_{18} \mathrm{H}_{24} \mathrm{NO}_{2}[\mathrm{M}+\mathrm{H}]^{+}: 286.1807$, found: 286.1813 .

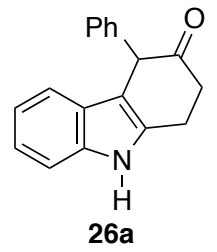

Indole 26a. The general procedure was followed by using $0.0724 \mathrm{~g}$ of styryl azide 24a $(0.250 \mathrm{mmol}), 0.0095 \mathrm{~g}$ of $\mathrm{Rh}_{2}(\mathrm{esp})_{2}(5 \mathrm{~mol} \%)$ and $2.5 \mathrm{~mL}$ of toluene. Purification by MPLC (3:97 to 60:40 EtOAc:hexanes) afforded the product as a white solid $(0.0549 \mathrm{~g}, 84 \%):{ }^{1} \mathrm{H}$ NMR $\left(500 \mathrm{MHz} \mathrm{CDCl}_{3}\right) \delta 8.03(\mathrm{~s}, 1 \mathrm{H}), 7.36(\mathrm{t}, J=8.2 \mathrm{~Hz}, 1 \mathrm{H}), 7.29(\mathrm{~m}, 5 \mathrm{H}), 7.17$ $(\mathrm{q}, J=7.8 \mathrm{~Hz}, 1 \mathrm{H}), 7.00(\mathrm{dq}, J=24.0 \mathrm{~Hz}, 7.8 \mathrm{~Hz}, 2 \mathrm{H}), 4.91(\mathrm{~s}, 1 \mathrm{H}), 3.26(\mathrm{~m}, 2 \mathrm{H}), 2.96(\mathrm{dq}, J=14.4 \mathrm{~Hz}, 7.6 \mathrm{~Hz}, 1 \mathrm{H})$, $2.60(\mathrm{~m}, 1 \mathrm{H}) ;{ }^{13} \mathrm{C}$ NMR $\left(125 \mathrm{MHz}, \mathrm{CDCl}_{3}\right) \delta 208.9(\mathrm{C}), 138.4(\mathrm{C}), 137.1(\mathrm{C}), 133.0(\mathrm{C}), 128.7(\mathrm{CH}), 128.3(\mathrm{CH}), 127.3$ $(\mathrm{CH}), 126.9(\mathrm{C}), 122.2(\mathrm{CH}), 119.8(\mathrm{CH}), 118.9(\mathrm{CH}), 110.8(\mathrm{CH}), 110.2(\mathrm{C}), 53.7(\mathrm{CH}), 35.5\left(\mathrm{CH}_{2}\right), 23.4\left(\mathrm{CH}_{2}\right)$; ATRFTIR (thin film): $3364,2891,1690,1451,1222 \mathrm{~cm}^{-1}$. HRMS (ES) $\mathrm{m} / z$ calculated for $\mathrm{C}_{18} \mathrm{H}_{16} \mathrm{NO}[\mathrm{M}+\mathrm{H}]^{+}: 262.1232$, found: 262.1230 . 


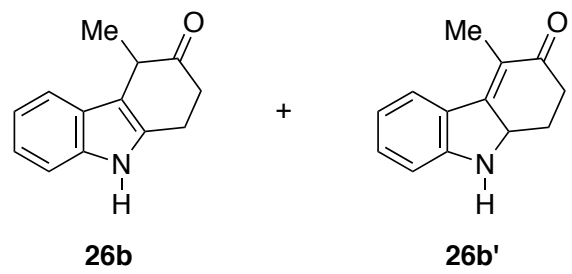

Indole 26b and 26b'. The general procedure was followed by using $0.0465 \mathrm{~g}$ of styryl azide $\mathbf{2 4 b}(0.200 \mathrm{mmol}), 0.0078 \mathrm{~g}$ of $\mathrm{Rh}_{2}(\mathrm{esp})_{2}(5 \mathrm{~mol} \%)$ and $2.0 \mathrm{~mL}$ of toluene. Purification by MPLC (3:97 to 60:40 EtOAc:hexanes) afforded the 5:1 mixture of products $\mathbf{2 6 \mathbf { b }}$ and $\mathbf{2 6 \mathbf { b }}$ ' respectively, as a colorless oil $(0.0347 \mathrm{~g}, 85 \%)$. Characterization data for $26 \mathbf{b}$ : ${ }^{1} \mathrm{H}$ NMR $\left(500 \mathrm{MHz}, \mathrm{CDCl}_{3}\right) \delta 7.98(\mathrm{~s}, 1 \mathrm{H}), 7.55(\mathrm{~d}, J=7.8 \mathrm{~Hz}, 1 \mathrm{H}), 7.32(\mathrm{~d}, J=8.0 \mathrm{~Hz}, 1 \mathrm{H}), 7.20(\mathrm{t}, J=7.6 \mathrm{~Hz}, 1 \mathrm{H}), 7.14$ $(\mathrm{t}, J=7.4 \mathrm{~Hz}, 1 \mathrm{H}), 3.77(\mathrm{q}, J=7.1 \mathrm{~Hz}, 1 \mathrm{H}), 3.13(\mathrm{qt}, J=17.3 \mathrm{~Hz}, 7.3 \mathrm{~Hz}, 2 \mathrm{H}), 2.89(\mathrm{dt}, J=13.7 \mathrm{~Hz}, 6.8 \mathrm{~Hz}, 1 \mathrm{H}), 2.75$ $(\mathrm{dt}, J=13.9 \mathrm{~Hz}, 7.0 \mathrm{~Hz}, 1 \mathrm{H}), 1.58(\mathrm{~d}, J=7.2 \mathrm{~Hz}, 3 \mathrm{H}) ;{ }^{13} \mathrm{C}$ NMR $\left(125 \mathrm{MHz}, \mathrm{CDCl}_{3}\right) \delta 212.9(\mathrm{C}), 136.9(\mathrm{C}), 131.3(\mathrm{C})$, $126.9(\mathrm{C}), 121.9(\mathrm{CH}), 119.7(\mathrm{CH}), 118.6(\mathrm{CH}), 112.9(\mathrm{C}), 111.0(\mathrm{CH}), 41.7(\mathrm{CH}), 36.9\left(\mathrm{CH}_{2}\right), 23.0\left(\mathrm{CH}_{2}\right), 18.0\left(\mathrm{CH}_{3}\right)$. Diagnostic peaks for 26b': ${ }^{1} \mathrm{H}$ NMR $\left(500 \mathrm{MHz}, \mathrm{CDCl}_{3}\right) \delta 7.41(\mathrm{~m}, 2 \mathrm{H}), 6.99(\mathrm{~d}, J=8.5 \mathrm{~Hz}, 1 \mathrm{H}), 5.17(\mathrm{~s}, 1 \mathrm{H}), 2.80(\mathrm{~s}$, $3 \mathrm{H}) ;{ }^{13} \mathrm{C}$ NMR $\left(125 \mathrm{MHz}, \mathrm{CDCl}_{3}\right) \delta 125.3(\mathrm{CH}), 114.9(\mathrm{CH}), 12.7\left(\mathrm{CH}_{3}\right)$. ATR-FTIR (thin film): 3399, 2924, 1699, $1455,1264 \mathrm{~cm}^{-1}$. HRMS (ES) $\mathrm{m} / z$ calculated for $\mathrm{C}_{13} \mathrm{H}_{14} \mathrm{NO}[\mathrm{M}+\mathrm{H}]^{+}: 200.1075$, found: 200.1075 .

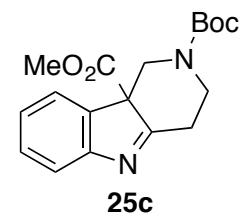

2-tert-Butyl-4a-methyl-4,4a-dihydro-1H-pyrido[3,4-b]indole-2,4a(3H)-dicarboxylate $25 \mathrm{c}$. The general procedure was followed by using $0.0242 \mathrm{~g}$ of styryl azide $24 \mathrm{c}(0.0680 \mathrm{mmol}), 0.0025 \mathrm{~g}$ of $\mathrm{Rh}_{2}(\mathrm{esp})_{2}(5 \mathrm{~mol} \%)$ and $0.7 \mathrm{~mL}$ of toluene at $120{ }^{\circ} \mathrm{C}$ for $24 \mathrm{~h}$. Purification by MPLC (3:97 to 30:70 EtOAc:hexanes) afforded the product, a yellow oil, as a mixture of rotamers $(0.0186 \mathrm{~g}, 83 \%):{ }^{1} \mathrm{H} \mathrm{NMR}\left(500 \mathrm{MHz}, \mathrm{CDCl}_{3}\right) \delta 7.84(\mathrm{~d}, J=7.3 \mathrm{~Hz}, 1 \mathrm{H}), 7.44(\mathrm{q}, J=6.6 \mathrm{~Hz}, 1 \mathrm{H}), 7.37(\mathrm{~m}, 2 \mathrm{H})$, $4.14(\mathrm{~d}, J=10.6 \mathrm{~Hz}, 1 \mathrm{H}), 3.99(\mathrm{~s}, 3 \mathrm{H}), 3.86(\mathrm{t}, J=10.9 \mathrm{~Hz}, 2 \mathrm{H}), 3.52(\mathrm{~d}, J=10.7 \mathrm{~Hz}, 0.4 \mathrm{H}), 3.42(\mathrm{~d}, J=10.5 \mathrm{~Hz}, 0.6 \mathrm{H})$, $2.95(\mathrm{q}, J=10.9 \mathrm{~Hz}, 1 \mathrm{H}), 1.82(\mathrm{~m}, 1 \mathrm{H}), 1.53(\mathrm{~s}, 3.5 \mathrm{H}), 1.44(\mathrm{~s}, 5.5 \mathrm{H}) ;{ }^{13} \mathrm{C}$ NMR $\left(125 \mathrm{MHz}, \mathrm{CDCl}_{3}\right) \delta 169.1(\mathrm{C}), 161.8$ $(\mathrm{C}), 154.6(\mathrm{C}), 151.9(\mathrm{C}), 145.3(\mathrm{C}), 129.3(\mathrm{CH}), 128.8(\mathrm{CH}), 123.8(\mathrm{CH}), 121.7(\mathrm{CH}), 80.0(\mathrm{C}), 62.8(\mathrm{C}), 52.9\left(\mathrm{CH}_{3}\right)$, $51.3\left(\mathrm{CH}_{2}\right), 45.0\left(\mathrm{CH}_{2}\right), 32.2\left(\mathrm{CH}_{2}\right), 28.4\left(\mathrm{CH}_{3}\right)$; ATR-FTIR (thin film): 2976, 1691, 1392, $1148,1120 \mathrm{~cm}^{-1}$. HRMS (EI) $\mathrm{m} / \mathrm{z}$ calculated for $\mathrm{C}_{18} \mathrm{H}_{23} \mathrm{~N}_{2} \mathrm{O}_{4}[\mathrm{M}+\mathrm{H}]^{+}: 331.1658$, found: 331.1662 .

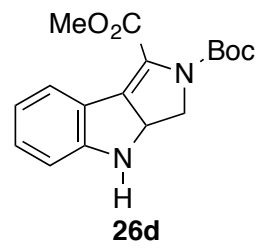

Alkylidene indoline 26d. The general procedure was followed by using $0.0507 \mathrm{~g}$ of styryl azide $24 \mathrm{~d}(0.147 \mathrm{mmol})$, $0.0056 \mathrm{~g}$ of $\mathrm{Rh}_{2}(\mathrm{esp})_{2}(5 \mathrm{~mol} \%)$ and $3.0 \mathrm{~mL}$ of toluene. Purification by MPLC (3:97 to 10:90 EtOAc:hexanes) afforded the product as a yellow solid $(0.0224 \mathrm{~g}, 48 \%)$ : ${ }^{1} \mathrm{H} \mathrm{NMR}\left(500 \mathrm{MHz}, \mathrm{CDCl}_{3}\right) \delta 7.79(\mathrm{~d}, J=7.6 \mathrm{~Hz}, 1 \mathrm{H}), 7.21(\mathrm{t}, J=7.7 \mathrm{~Hz}$, $1 \mathrm{H}), 6.87(\mathrm{t}, J=7.6 \mathrm{~Hz}, 1 \mathrm{H}), 6.79(\mathrm{~d}, J=8.0 \mathrm{~Hz}, 1 \mathrm{H}), 5.09(\mathrm{~m}, 1 \mathrm{H}), 4.48(\mathrm{dd}, J=10.7 \mathrm{~Hz}, 7.5 \mathrm{~Hz}, 1 \mathrm{H}), 4.31(\mathrm{~s}, 1 \mathrm{H}), 3.86$ (s, 3H), $3.84(\mathrm{t}, J=11.5 \mathrm{~Hz}, 1 \mathrm{H}), 1.48(\mathrm{~s}, 9 \mathrm{H}) ;{ }^{13} \mathrm{C}$ NMR $\left(125 \mathrm{MHz}, \mathrm{CDCl}_{3}\right) \delta 163.9(\mathrm{C}), 161.8(\mathrm{C}), 159.4(\mathrm{C}), 153.5(\mathrm{C})$, $144.2(\mathrm{C}), 131.6(\mathrm{CH}), 125.8(\mathrm{CH}), 123.7(\mathrm{C}), 120.5(\mathrm{CH}), 111.7(\mathrm{CH}), 81.1(\mathrm{C}), 66.5\left(\mathrm{CH}_{3}\right), 60.6\left(\mathrm{CH}_{2}\right), 51.8(\mathrm{CH}), 28.2$ $\left(\mathrm{CH}_{3}\right)$; ATR-FTIR (thin film): 3309, 2979, 1738, 1698, 1627, 1249, $1164 \mathrm{~cm}^{-1}$. HRMS (ES) $\mathrm{m} / z$ calculated for $\mathrm{C}_{17} \mathrm{H}_{20} \mathrm{~N}_{2} \mathrm{O}_{4} \mathrm{Na}[\mathrm{M}+\mathrm{Na}]^{+}: 339.1321$, found: 339.1321 . 


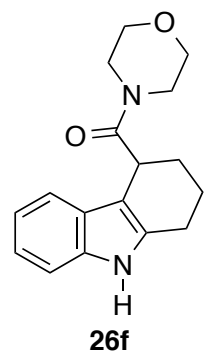

Indole 26f. The general procedure was followed by using $0.0436 \mathrm{~g}$ of styryl azide $24 \mathbf{f}(0.140 \mathrm{mmol}), 0.0053 \mathrm{~g}$ of $\mathrm{Rh}_{2}(\mathrm{esp})_{2}(5 \mathrm{~mol} \%)$ and $1.5 \mathrm{~mL}$ of toluene. Purification by MPLC (3:97 to 90:10 EtOAc:hexanes) afforded the product as a white solid $(0.0103 \mathrm{~g}, 26 \%):{ }^{1} \mathrm{H}$ NMR $\left(500 \mathrm{MHz}, \mathrm{CDCl}_{3}\right) \delta 7.90(\mathrm{~s}, 1 \mathrm{H}), 7.27(\mathrm{~d}, J=9.2 \mathrm{~Hz}, 1 \mathrm{H}), 7.25(\mathrm{~d}, J=8.1 \mathrm{~Hz}$, $1 \mathrm{H}), 7.09(\mathrm{t}, J=7.5 \mathrm{~Hz}, 1 \mathrm{H}), 7.04(\mathrm{t}, J=7.4 \mathrm{~Hz}, 1 \mathrm{H}), 4.17(\mathrm{dd}, J=7.8 \mathrm{~Hz}, 5.4 \mathrm{~Hz}, 1 \mathrm{H}), 3.77(\mathrm{~m}, 2 \mathrm{H}), 3.63(\mathrm{~m}, 5 \mathrm{H}), 3.46$ $(\mathrm{m}, 1 \mathrm{H}), 2.73(\mathrm{~m}, 2 \mathrm{H}), 2.17(\mathrm{tq}, J=10.4 \mathrm{~Hz}, 5.3 \mathrm{~Hz}, 2 \mathrm{H}), 1.91(\mathrm{q}, J=10.1 \mathrm{~Hz}, 1 \mathrm{H}), 1.83(\mathrm{ddd}, J=18.6 \mathrm{~Hz}, 11.1 \mathrm{~Hz}, 6.6$ $\mathrm{Hz}, 1 \mathrm{H}) ;{ }^{13} \mathrm{C}$ NMR $\left(125 \mathrm{MHz}, \mathrm{CDCl}_{3}\right) \delta 173.4(\mathrm{C}), 135.8(\mathrm{C}), 134.7(\mathrm{C}), 126.6(\mathrm{C}), 121.4(\mathrm{CH}), 119.6(\mathrm{CH}), 117.9(\mathrm{CH})$, $110.7(\mathrm{CH}), 108.5(\mathrm{C}), 67.2\left(\mathrm{CH}_{2}\right), 66.7\left(\mathrm{CH}_{2}\right), 46.4\left(\mathrm{CH}_{2}\right), 42.6\left(\mathrm{CH}_{2}\right), 38.0(\mathrm{CH}), 27.5\left(\mathrm{CH}_{2}\right), 22.9\left(\mathrm{CH}_{2}\right), 21.7\left(\mathrm{CH}_{2}\right)$; ATR-FTIR (thin film): 3305, 3054, 2926, 2857, 1715, 1620, 1435, 1264, 1227, $1113 \mathrm{~cm}^{-1}$. HRMS (ES) $\mathrm{m} / z$ calculated for $\mathrm{C}_{17} \mathrm{H}_{21} \mathrm{~N}_{2} \mathrm{O}_{2}[\mathrm{M}+\mathrm{H}]^{+}:$285.1603, found: 285.1606.

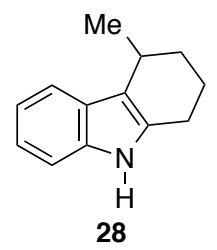

Indole 28. The general procedure was followed by using $0.0329 \mathrm{~g}$ of styryl azide $27(0.154 \mathrm{mmol}), 0.0058 \mathrm{~g}_{\mathrm{of}} \mathrm{Rh}_{2}(\mathrm{esp})_{2}$ $(5 \mathrm{~mol} \%)$ and $1.6 \mathrm{~mL}$ of toluene. Purification by MPLC (3:97 to 60:40 EtOAc:hexanes) afforded the product as a yellow oil $(0.0191 \mathrm{~g}, 67 \%):{ }^{1} \mathrm{H}$ NMR $\left(500 \mathrm{MHz}, \mathrm{CDCl}_{3}\right) \delta 7.65(\mathrm{~s}, 1 \mathrm{H}), 7.57(\mathrm{~d}, J=8.0 \mathrm{~Hz}, 1 \mathrm{H}), 7.29(\mathrm{~d}, J=7.5 \mathrm{~Hz}, 1 \mathrm{H}), 7.12-$ $7.05(\mathrm{~m}, 2 \mathrm{H}), 3.12(\mathrm{~m}, 1 \mathrm{H}), 2.70(\mathrm{~m}, 2 \mathrm{H}), 2.00(\mathrm{~m}, 2 \mathrm{H}), 1.82(\mathrm{~m}, 1 \mathrm{H}), 1.59(\mathrm{~m}, 1 \mathrm{H}), 1.37(\mathrm{~d}, J=7.0 \mathrm{~Hz}, 3 \mathrm{H}) ;{ }^{13} \mathrm{C} \mathrm{NMR}$ $\left(125 \mathrm{MHz}, \mathrm{CDCl}_{3}\right) \delta 135.8(\mathrm{C}), 133.9(\mathrm{C}), 127.4(\mathrm{C}), 120.8(\mathrm{CH}), 119.0(\mathrm{CH}), 118.7(\mathrm{CH}), 115.1(\mathrm{C}), 110.5(\mathrm{CH}), 32.1$ $\left(\mathrm{CH}_{2}\right), 27.1(\mathrm{CH}), 23.5\left(\mathrm{CH}_{2}\right), 21.3\left(\mathrm{CH}_{3}\right), 20.4\left(\mathrm{CH}_{2}\right)$; ATR-FTIR (thin film): 3325, 2930, 2896, 1667, 1615, $1455 \mathrm{~cm}^{-1}$. HRMS (ES) $m / z$ calculated for $\mathrm{C}_{13} \mathrm{H}_{14} \mathrm{~N}[\mathrm{M}-\mathrm{H}]^{+}: 184.1126$, found: 184.1135 .

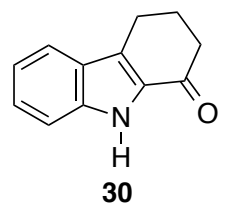

Indole 30. The general procedure was followed by using $0.0400 \mathrm{~g}$ of styryl azide $29(0.188 \mathrm{mmol}), 0.0071 \mathrm{~g}$ of $\mathrm{Rh}_{2}(\mathrm{esp})_{2}$ $(5 \mathrm{~mol} \%)$ and $1.9 \mathrm{~mL}$ of toluene. Purification by MPLC (3:97 to 60:40 EtOAc:hexanes) afforded the product as a white solid $(0.0285 \mathrm{~g}, 82 \%):{ }^{1} \mathrm{H}$ NMR $\left(500 \mathrm{MHz}, \mathrm{CDCl}_{3}\right) \delta 9.42(\mathrm{~s}, 1 \mathrm{H}), 7.67(\mathrm{~d}, J=8.1 \mathrm{~Hz}, 1 \mathrm{H}), 7.44(\mathrm{~d}, J=8.4 \mathrm{~Hz}, 1 \mathrm{H}), 7.38$ $(\mathrm{t}, J=7.6 \mathrm{~Hz}, 1 \mathrm{H}), 7.16(\mathrm{t}, J=7.5 \mathrm{~Hz}, 1 \mathrm{H}), 3.02(\mathrm{t}, J=6.1 \mathrm{~Hz}, 2 \mathrm{H}), 2.71(\mathrm{t}, J=6.5 \mathrm{~Hz}, 2 \mathrm{H}), 2.28$ (quintet, $J=6.3 \mathrm{~Hz}$, 2H); ${ }^{13} \mathrm{C}$ NMR $\left(125 \mathrm{MHz}, \mathrm{CDCl}_{3}\right) \delta 191.8(\mathrm{C}), 138.1(\mathrm{C}), 131.3(\mathrm{C}), 129.7(\mathrm{C}), 127.1(\mathrm{CH}), 125.9(\mathrm{C}), 121.4(\mathrm{CH}), 120.4$ $(\mathrm{CH}), 112.7(\mathrm{CH}), 38.3\left(\mathrm{CH}_{2}\right), 25.0\left(\mathrm{CH}_{2}\right), 21.5\left(\mathrm{CH}_{2}\right)$; ATR-FTIR (thin film): 3279, 2925, 2860, 1634, $1615,1243 \mathrm{~cm}^{-1}$. HRMS (ES) $m / z$ calculated for $\mathrm{C}_{12} \mathrm{H}_{12} \mathrm{NO}[\mathrm{M}+\mathrm{H}]^{+}: 186.0919$, found: 186.0924 . 

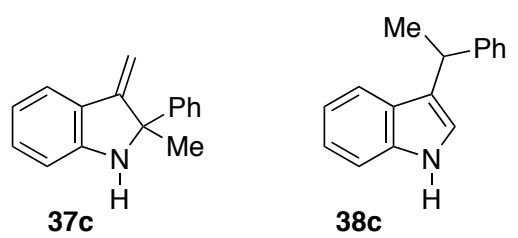

Alkylidene indoline 37c and Indole 38c. The general procedure was followed by using $0.0458 \mathrm{~g}$ of styryl azide 35c $(0.184 \mathrm{mmol}), 0.0070 \mathrm{~g}$ of $\mathrm{Rh}_{2}(\mathrm{esp})_{2}(5 \mathrm{~mol} \%)$ and $1.8 \mathrm{~mL}$ of toluene. Purification by MPLC (3:97 to $60: 40$ EtOAc:hexanes) with alumina afforded a mixture of the products $\mathbf{3 8 c}$ as a yellow solid $(0.0332 \mathrm{~g}, 82 \%)$ and $37 \mathrm{c}$ as a colorless oil $(0.0051 \mathrm{~g}, 13 \%)$. Characterization data for 37c: ${ }^{1} \mathrm{H}$ NMR $\left(500 \mathrm{MHz}, \mathrm{CDCl}_{3}\right) \delta 7.47(\mathrm{~d}, J=7.3 \mathrm{~Hz}, 2 \mathrm{H}), 7.38$ $(\mathrm{d}, J=7.5 \mathrm{~Hz}, 1 \mathrm{H}), 7.32(\mathrm{t}, J=7.6 \mathrm{~Hz}, 2 \mathrm{H}), 7.24(\mathrm{t}, J=7.4 \mathrm{~Hz}, 1 \mathrm{H}), 7.17(\mathrm{t}, J=7.6 \mathrm{~Hz}, 1 \mathrm{H}), 6.78(\mathrm{t}, J=7.5 \mathrm{~Hz}, 1 \mathrm{H})$, $6.71(\mathrm{~d}, J=8.0 \mathrm{~Hz}, 1 \mathrm{H}), 5.42(\mathrm{~s}, 1 \mathrm{H}), 4.72(\mathrm{~s}, 1 \mathrm{H}), 4.30(\mathrm{~s}, 1 \mathrm{H}), 1.77(\mathrm{~s}, 3 \mathrm{H}) ;{ }^{13} \mathrm{C} \mathrm{NMR}\left(125 \mathrm{MHz}, \mathrm{CDCl}_{3}\right) \delta 155.1(\mathrm{C})$, $150.9(\mathrm{C}), 146.8(\mathrm{C}), 130.3(\mathrm{CH}), 128.4(\mathrm{CH}), 127.0(\mathrm{CH}), 125.6(\mathrm{CH}), 121.4(\mathrm{CH}), 118.8(\mathrm{CH}), 110.1(\mathrm{CH}), 101.3$ $\left(\mathrm{CH}_{2}\right), 67.9(\mathrm{C}), 28.5\left(\mathrm{CH}_{3}\right)$, only visible peaks; ATR-FTIR (thin film): 3375, 2971, 1607, $1468 \mathrm{~cm}^{-1}$; HRMS (ES) $\mathrm{m} / \mathrm{z}$ calculated for $\mathrm{C}_{16} \mathrm{H}_{16} \mathrm{~N}[\mathrm{M}+\mathrm{H}]^{+}: 222.1283$, found: 222.1289 .

Characterization data for 38c: ${ }^{1} \mathrm{H}$ NMR $\left(500 \mathrm{MHz}, \mathrm{CDCl}_{3}\right) \delta 7.91(\mathrm{~s}, 1 \mathrm{H}), 7.40(\mathrm{~d}, J=8.0 \mathrm{~Hz}, 1 \mathrm{H}), 7.32(\mathrm{~m}, 5 \mathrm{H}), 7.20(\mathrm{~m}$, $2 \mathrm{H}), 7.04(\mathrm{t}, J=7.5 \mathrm{~Hz}, 1 \mathrm{H}), 7.01(\mathrm{~d}, J=1.5 \mathrm{~Hz}, 1 \mathrm{H}), 4.41(\mathrm{q}, J=7.1 \mathrm{~Hz}, 1 \mathrm{H}), 1.74(\mathrm{~d}, J=7.2 \mathrm{~Hz}, 3 \mathrm{H}) ;{ }^{13} \mathrm{C}$ NMR $(125$ $\left.\mathrm{MHz}, \mathrm{CDCl}_{3}\right) \delta 146.9(\mathrm{C}), 136.7(\mathrm{C}), 128.4(\mathrm{CH}), 127.5(\mathrm{CH}), 126.9(\mathrm{C}), 126.0(\mathrm{CH}), 122.0(\mathrm{CH}), 121.5(\mathrm{C}), 121.1(\mathrm{CH})$, $119.8(\mathrm{CH}), 119.3(\mathrm{CH}), 111.1(\mathrm{CH}), 37.0(\mathrm{CH}), 22.5\left(\mathrm{CH}_{3}\right)$. ATR-FTIR (thin film): 3398, 2962, $1451,1223 \mathrm{~cm}^{-1}$; HRMS (ES) $m / z$ calculated for $\mathrm{C}_{16} \mathrm{H}_{16} \mathrm{~N}[\mathrm{M}+\mathrm{H}]^{+}: 222.1283$, found: 222.1290 .
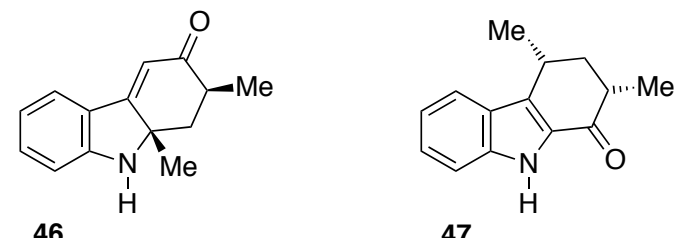

Alkylidene indoline 46 and Indole 47. The general procedure was followed by using $0.0309 \mathrm{~g}$ of styryl azide $45(0.128$ $\mathrm{mmol}), 0.0048 \mathrm{~g}$ of $\mathrm{Rh}_{2}(\mathrm{esp})_{2}(5 \mathrm{~mol} \%)$ and $1.3 \mathrm{~mL}$ of toluene. Purification by MPLC (3:97 to 60:40 EtOAc:hexanes) afforded 46, a yellow oil, as a 6:1 mixture of diastereomers $(0.0103 \mathrm{~g}, 38 \%)$ and $\mathbf{4 7}$, a white solid, as a $>20: 1$ mixture of diastereomers $(0.0120 \mathrm{~g}, 44 \%)$. The identity of each of the diastereomers was determined by nOe. Characterization data for the major diastereomer of 46: ${ }^{1} \mathrm{H}$ NMR $\left(500 \mathrm{MHz}, \mathrm{CDCl}_{3}\right) \delta 7.46(\mathrm{~d}, J=7.9 \mathrm{~Hz}, 1 \mathrm{H}), 7.30(\mathrm{t}, J=7.7 \mathrm{~Hz}, 1 \mathrm{H}), 6.85(\mathrm{t}$, $J=7.5 \mathrm{~Hz}, 1 \mathrm{H}), 6.79(\mathrm{~d}, J=8.0 \mathrm{~Hz}, 1 \mathrm{H}), 6.13(\mathrm{~s}, 1 \mathrm{H}), 4.24(\mathrm{~s}, 1 \mathrm{H}), 2.50$ (dquintet, $J=12.6 \mathrm{~Hz}, 6.3 \mathrm{~Hz}, 1 \mathrm{H}), 2.30(\mathrm{dd}, J=$ $12.5 \mathrm{~Hz}, 5.0 \mathrm{~Hz}, 1 \mathrm{H}), 1.94(\mathrm{t}, J=12.6 \mathrm{~Hz}, 1 \mathrm{H}), 1.49(\mathrm{~s}, 3 \mathrm{H}), 1.25(\mathrm{~d}, J=6.8 \mathrm{~Hz}, 3 \mathrm{H}) ;{ }^{13} \mathrm{C} \mathrm{NMR}\left(125 \mathrm{MHz}, \mathrm{CDCl}_{3}\right) \delta$ $200.2(\mathrm{C}), 153.7(\mathrm{C}), 148.4(\mathrm{C}), 133.6(\mathrm{CH}), 123.9(\mathrm{CH}), 122.8(\mathrm{C}), 119.9(\mathrm{CH}), 114.0(\mathrm{CH}), 111.9(\mathrm{CH}), 63.5(\mathrm{C}), 44.2$ $\left(\mathrm{CH}_{2}\right)$, $38.4(\mathrm{CH}), 26.6\left(\mathrm{CH}_{3}\right), 17.0\left(\mathrm{CH}_{3}\right)$. Characterization data for the minor diastereomer: ${ }^{1} \mathrm{H} \mathrm{NMR}\left(500 \mathrm{MHz}, \mathrm{CDCl}_{3}\right)$ $\delta 6.18(\mathrm{~s}, 1 \mathrm{H}), 1.40(\mathrm{~d}, J=8.0 \mathrm{~Hz}, 3 \mathrm{H}) ;{ }^{13} \mathrm{C}$ NMR $\left(125 \mathrm{MHz}, \mathrm{CDCl}_{3}\right) \delta 119.8(\mathrm{CH}), 113.4(\mathrm{CH}), 40.3(\mathrm{CH})$. ATR-FTIR (thin film): $3322,2927,1610,1264 \mathrm{~cm}^{-1}$. HRMS (ES) $\mathrm{m} / z$ calculated for $\mathrm{C}_{14} \mathrm{H}_{16} \mathrm{NO}[\mathrm{M}+\mathrm{H}]^{+}: 214.1232$, found: 214.1236 .

Characterization data for 47: ${ }^{1} \mathrm{H}$ NMR $\left(500 \mathrm{MHz} \mathrm{CDCl}_{3}\right) \delta 9.30(\mathrm{~s}, 1 \mathrm{H}), 7.87(\mathrm{~d}, J=8.2 \mathrm{~Hz}, 1 \mathrm{H}), 7.45(\mathrm{~d}, J=8.3 \mathrm{~Hz}$, $1 \mathrm{H}), 7.35(\mathrm{t}, J=7.6 \mathrm{~Hz}, 1 \mathrm{H}), 7.13(\mathrm{t}, J=7.6 \mathrm{~Hz}, 1 \mathrm{H}), 3.40(\mathrm{~m}, 1 \mathrm{H}), 2.71(\mathrm{dqd}, J=13.3 \mathrm{~Hz}, 7.7 \mathrm{~Hz}, 4.4 \mathrm{~Hz}, 1 \mathrm{H}), 2.25(\mathrm{dt}$, $J=13.1 \mathrm{~Hz}, 4.4 \mathrm{~Hz}, 1 \mathrm{H}), 1.76(\mathrm{dt}, J=13.0 \mathrm{~Hz}, 11.5 \mathrm{~Hz}, 1 \mathrm{H}), 1.61(\mathrm{~d}, J=6.8 \mathrm{~Hz}, 3 \mathrm{H}), 1.32(\mathrm{~d}, J=6.8 \mathrm{~Hz}, 3 \mathrm{H}) ;{ }^{13} \mathrm{C} \mathrm{NMR}$ $\left(125 \mathrm{MHz}, \mathrm{CDCl}_{3}\right) \delta 194.3(\mathrm{C}), 138.3(\mathrm{C}), 132.4(\mathrm{C}), 130.8(\mathrm{C}), 126.4(\mathrm{CH}), 125.8(\mathrm{C}), 122.7(\mathrm{CH}), 120.2(\mathrm{CH}), 112.8$ $(\mathrm{CH}), 43.4\left(\mathrm{CH}_{2}\right), 41.7(\mathrm{CH}), 30.0(\mathrm{CH}), 20.9\left(\mathrm{CH}_{3}\right), 15.0\left(\mathrm{CH}_{3}\right)$; ATR-FTIR (thin film): 3270, 2958, 2922, 2852, 1634, $1580,1407 \mathrm{~cm}^{-1}$. HRMS (ES) $\mathrm{m} / z$ calculated for $\mathrm{C}_{14} \mathrm{H}_{16} \mathrm{NO}[\mathrm{M}+\mathrm{H}]^{+}:$214.1232, found: 214.1236 . 


\section{Mechanistic Study for $\mathrm{Rh}_{2}$ (II)-Catalyzed Electrocyclization and $\mathrm{sp}^{3} \mathrm{C}-\mathrm{H}$ Bond Amination .}

\section{A. Hammett study: effect of changing the electronic nature of the allylic $\mathrm{C}-\mathrm{H}$ bond on the reaction} outcome.<smiles>C/C=C(\c1ccccc1N)C(C)c1ccc(OC)cc1</smiles>

$33 a$

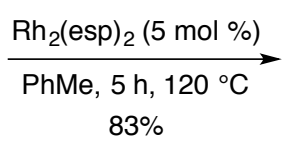

$83 \%$

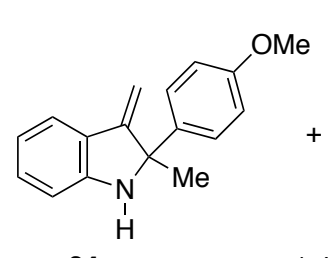

$1: 4$

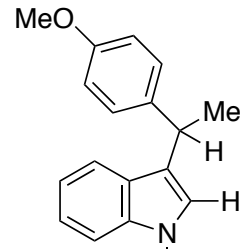

35a $\mathrm{H}$

To a mixture of $0.0222 \mathrm{~g}$ of styryl azide 33a $(0.0790 \mathrm{mmol})$ and $0.0038 \mathrm{~g}$ of $\mathrm{Rh}_{2}(\mathrm{esp})_{2}(0.0039 \mathrm{mmol})$ in a Schlenk tube was added $1.6 \mathrm{~mL}$ of toluene. The resulting mixture was heated to $120{ }^{\circ} \mathrm{C}$. After $5 \mathrm{~h}$, the mixture was cooled to room temperature, diluted with $\mathrm{CH}_{2} \mathrm{Cl}_{2}$ and concentrated in vacuo to afford a mixture of 34a and 35a. The resulting residue was dissolved in $0.5 \mathrm{~mL}$ of $\mathrm{CDCl}_{3}$ and $7 \mu \mathrm{L}$ of dibromomethane $(0.1 \mathrm{mmol})$ was added. The area of the olefin $\mathrm{C}-\mathrm{H}$ peak of 34a was compared with benzylic $\mathrm{C}-\mathrm{H}$ peak of $\mathbf{3 5 a}$ to derive a ratio of $\mathbf{3 4 a}: \mathbf{3 5 a}$ (1:4).<smiles>Cc1ccc(C(C)/C(=C/c2ccccc2N)C(C)(C)C)cc1</smiles>

33b

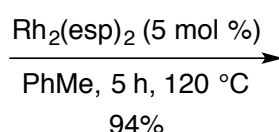

$94 \%$

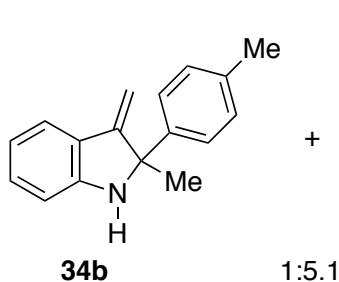

$1: 5.1$

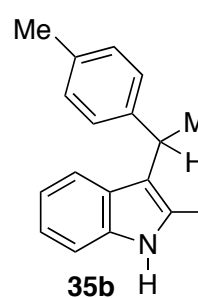

(s32)

To a mixture of $0.0225 \mathrm{~g}$ of styryl azide $33 \mathrm{~b}(0.0850 \mathrm{mmol})$ and $0.0032 \mathrm{~g}$ of $\mathrm{Rh}_{2}(\mathrm{esp})_{2}(0.0042 \mathrm{mmol})$ in a Schlenk tube was added $1.7 \mathrm{~mL}$ of toluene. The resulting mixture was heated to $120{ }^{\circ} \mathrm{C}$. After $5 \mathrm{~h}$, the mixture was cooled to room temperature, diluted with $\mathrm{CH}_{2} \mathrm{Cl}_{2}$ and concentrated in vacuo to afford a mixture of $\mathbf{3 4 \mathbf { b }}$ and $\mathbf{3 5 \mathbf { b }}$. The resulting residue was dissolved in $0.5 \mathrm{~mL}$ of $\mathrm{CDCl}_{3}$ and $7 \mu \mathrm{L}$ of dibromomethane $(0.1 \mathrm{mmol})$ was added. The area of the olefin $\mathrm{C}-\mathrm{H}$ peak of $\mathbf{3 4 b}$ was compared with benzylic $\mathrm{C}-\mathrm{H}$ peak of $\mathbf{3 5 b}$ to derive a ratio of $\mathbf{3 4 \mathbf { b } : 3 5 \mathbf { b }}$ (1:5.1).

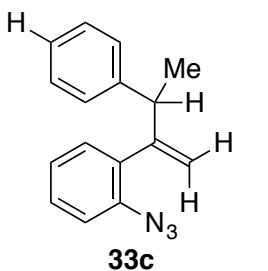

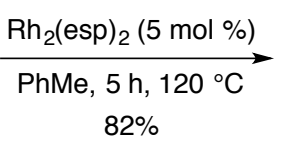

$82 \%$
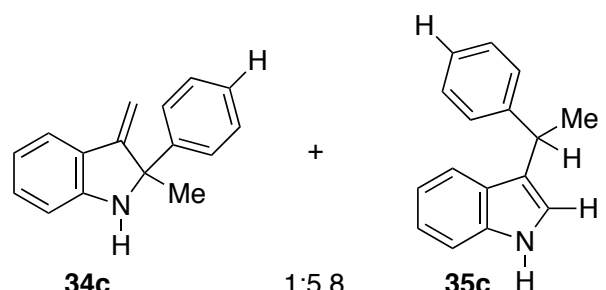

(s33)

To a mixture of $0.013 \mathrm{~g}$ of styryl azide $33 \mathrm{c}(0.052 \mathrm{mmol})$ and $0.0019 \mathrm{~g}$ of $\mathrm{Rh}_{2}(\mathrm{esp})_{2}(0.0026 \mathrm{mmol})$ in a Schlenk tube was added $1.0 \mathrm{~mL}$ of toluene. The resulting mixture was heated to $120{ }^{\circ} \mathrm{C}$. After $5 \mathrm{~h}$, the mixture was cooled to room temperature, diluted with $\mathrm{CH}_{2} \mathrm{Cl}_{2}$ and concentrated in vacuo to afford a mixture of $\mathbf{3 4 c}$ and $\mathbf{3 5 c}$. The resulting residue was dissolved in $0.5 \mathrm{~mL}$ of $\mathrm{CDCl}_{3}$ and $7 \mu \mathrm{L}$ of dibromomethane $(0.1 \mathrm{mmol})$ was added. The area of the olefin $\mathrm{C}-\mathrm{H}$ peak of $\mathbf{3 4 c}$ was compared with benzylic $\mathrm{C}-\mathrm{H}$ peak of $\mathbf{3 5 c}$ to derive a ratio of $\mathbf{3 4 c}: \mathbf{3 5 c}$ (1:5.8). 


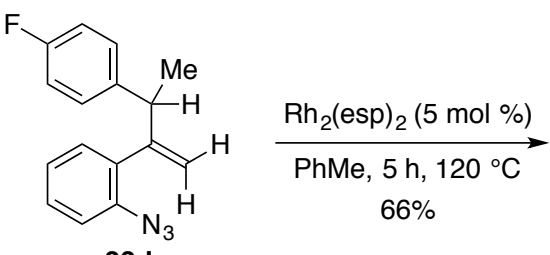

33d
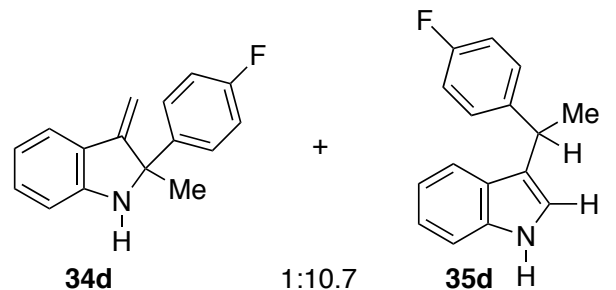

To a mixture of $0.0125 \mathrm{~g}$ of styryl azide $33 \mathrm{~d}(0.0460 \mathrm{mmol})$ and $0.0018 \mathrm{~g}$ of $\mathrm{Rh}_{2}(\mathrm{esp})_{2}(0.0023 \mathrm{mmol})$ in a Schlenk tube was added $0.90 \mathrm{~mL}$ of toluene. The resulting mixture was heated to $120^{\circ} \mathrm{C}$. After $5 \mathrm{~h}$, the mixture was cooled to room temperature, diluted with $\mathrm{CH}_{2} \mathrm{Cl}_{2}$ and concentrated in vacuo to afford a mixture of $\mathbf{3 4 d}$ and $\mathbf{3 5 d}$. The resulting residue was dissolved in $0.5 \mathrm{~mL}$ of $\mathrm{CDCl}_{3}$ and $7 \mu \mathrm{L}$ of dibromomethane $(0.1 \mathrm{mmol})$ was added. The area of the olefin $\mathrm{C}-\mathrm{H}$ peak of $\mathbf{3 4 d}$ was compared with benzylic $\mathrm{C}-\mathrm{H}$ peak of $\mathbf{3 5 d}$ to derive a ratio of $\mathbf{3 4 d}: \mathbf{3 5 d}(1: 10.7)$.
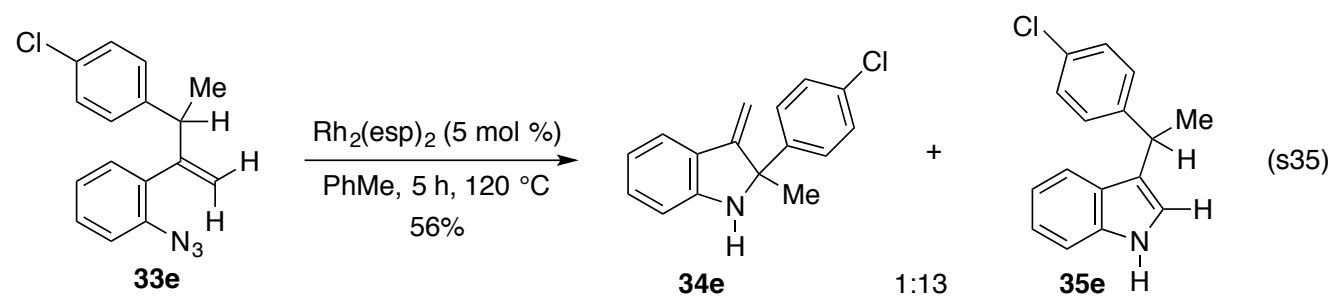

To a mixture of $0.0118 \mathrm{~g}$ of styryl azide 33e $(0.0420 \mathrm{mmol})$ and $0.0016 \mathrm{~g}$ of $\mathrm{Rh}_{2}(\mathrm{esp})_{2}(0.0021 \mathrm{mmol})$ in a Schlenk tube was added $0.80 \mathrm{~mL}$ of toluene. The resulting mixture was heated to $120^{\circ} \mathrm{C}$. After $5 \mathrm{~h}$, the mixture was cooled to room temperature, diluted with $\mathrm{CH}_{2} \mathrm{Cl}_{2}$ and concentrated in vacuo to afford a mixture of $\mathbf{3 4 e}$ and $35 \mathbf{e}$. The resulting residue was dissolved in $0.5 \mathrm{~mL}$ of $\mathrm{CDCl}_{3}$ and $7 \mu \mathrm{L}$ of dibromomethane $(0.1 \mathrm{mmol})$ was added. The area of the olefin $\mathrm{C}-\mathrm{H}$ peak of 34e was compared with benzylic $\mathrm{C}-\mathrm{H}$ peak of $35 \mathbf{e}$ to derive a ratio of $\mathbf{3 4 \mathbf { e }}: 35 \mathbf{e}$ (1:13.0).

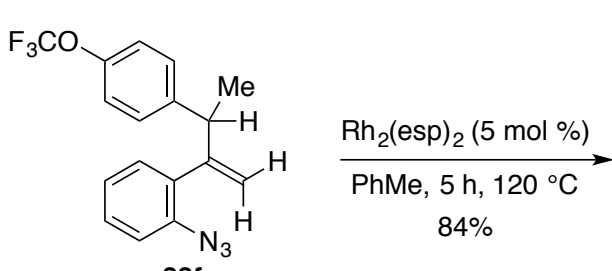

$33 f$
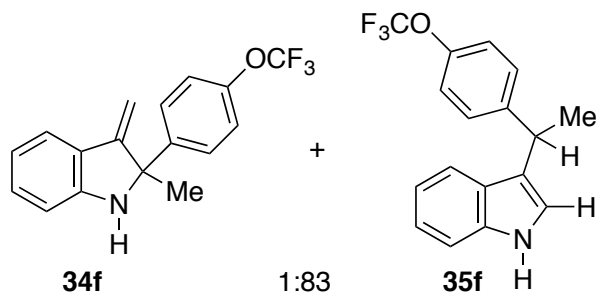

(s36)

To a mixture of $0.0343 \mathrm{~g}$ of styryl azide $33 \mathrm{f}(0.103 \mathrm{mmol})$ and $0.0039 \mathrm{~g}$ of $\mathrm{Rh}_{2}(\mathrm{esp})_{2}(0.0051 \mathrm{mmol})$ in a Schlenk tube was added $2.0 \mathrm{~mL}$ of toluene. The resulting mixture was heated to $120{ }^{\circ} \mathrm{C}$. After $5 \mathrm{~h}$, the mixture was cooled to room temperature, diluted with $\mathrm{CH}_{2} \mathrm{Cl}_{2}$ and concentrated in vacuo to afford a mixture of $\mathbf{3 4 f}$ and $\mathbf{3 5 f}$. The resulting residue was dissolved in $0.5 \mathrm{~mL}$ of $\mathrm{CDCl}_{3}$ and $7 \mu \mathrm{L}$ of dibromomethane $(0.1 \mathrm{mmol})$ was added. The area of the olefin $\mathrm{C}-\mathrm{H}$ peak of $\mathbf{3 4 f}$ was compared with benzylic $\mathrm{C}-\mathrm{H}$ peak of $\mathbf{3 5 f}$ to derive a ratio of $\mathbf{3 4 f}: \mathbf{3 5 f}$ (1:83). 
Table s3. Effect of changing the electronic environment at the allylic $\mathrm{C}-\mathrm{H}$ bond reaction center.

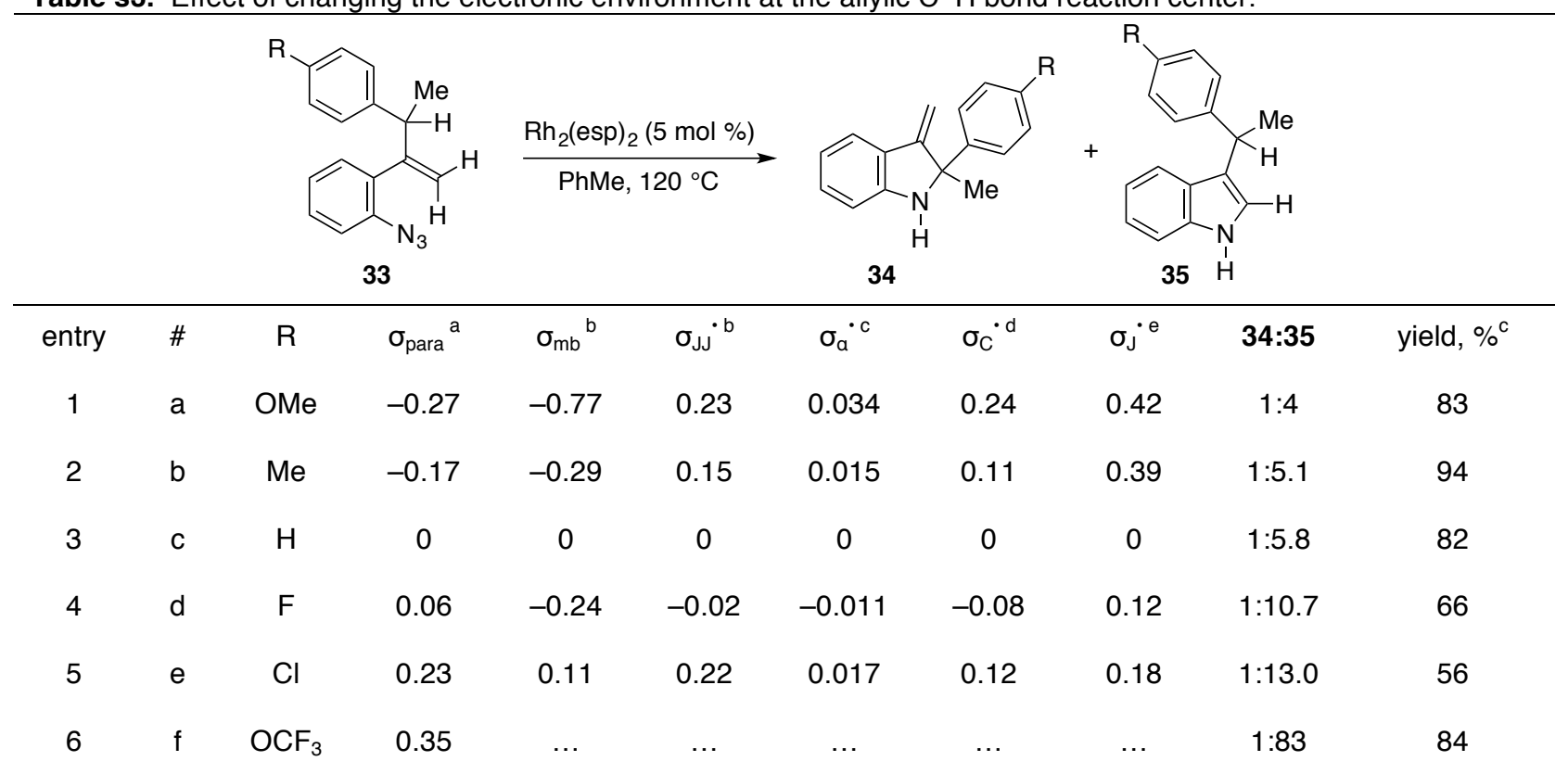

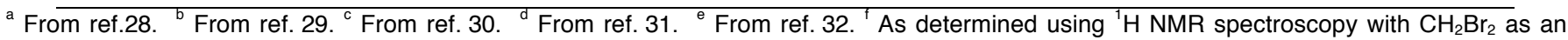
internal standard.

Figure s1. Effect of changing the electronic environment at the allylic $\mathrm{C}-\mathrm{H}$ bond reaction center: correlation of heterocycle product ratios with Hammett $\sigma_{\text {para }}$ values.

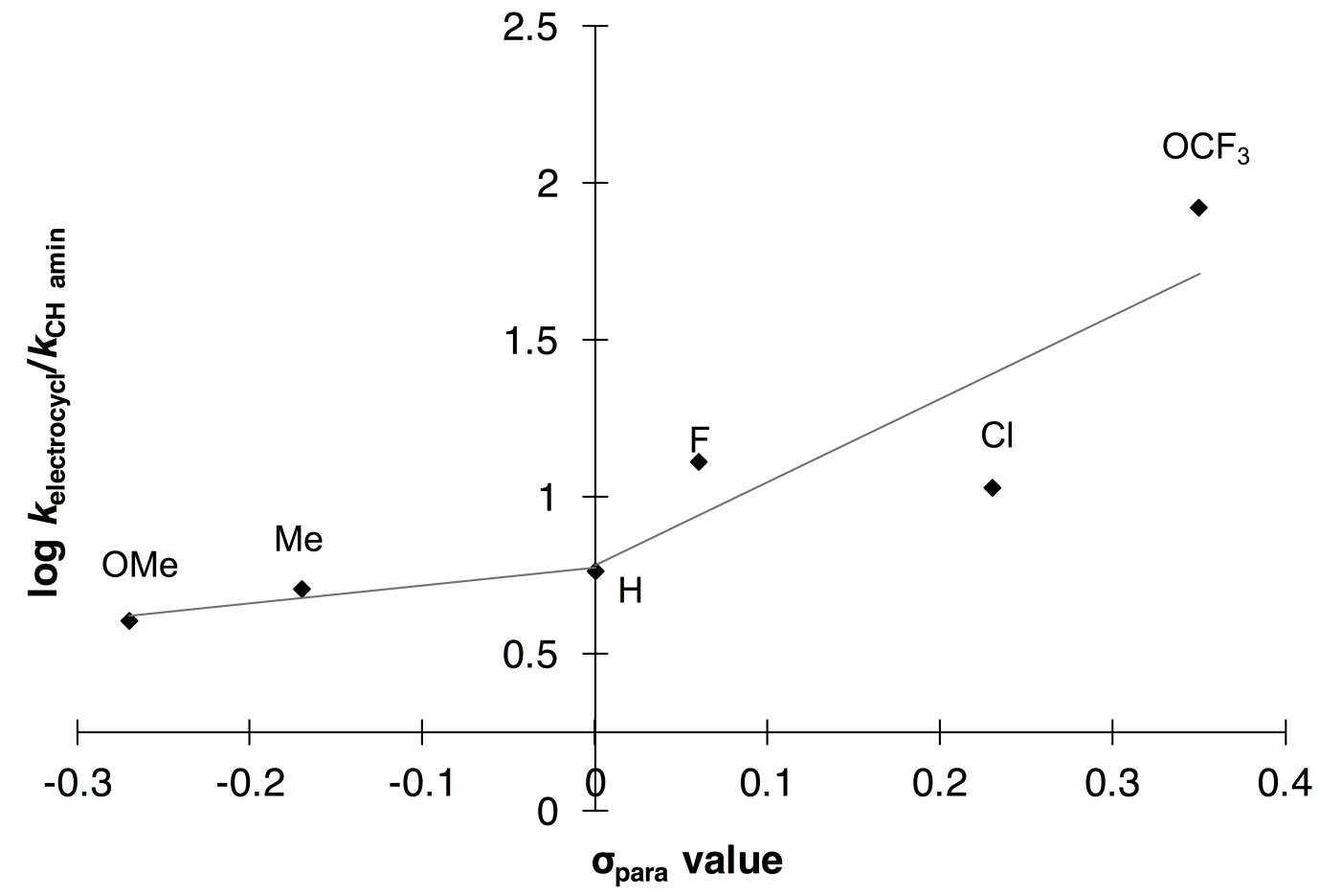


Figure s2. Correlation of heterocycle product ratios with Jiang and $\mathrm{Ji} \sigma_{\mathrm{mb}}$ and $\sigma_{\mathrm{JJ}}$ 'values: substituent effect observed at the allylic $\mathrm{C}-\mathrm{H}$ reaction site.

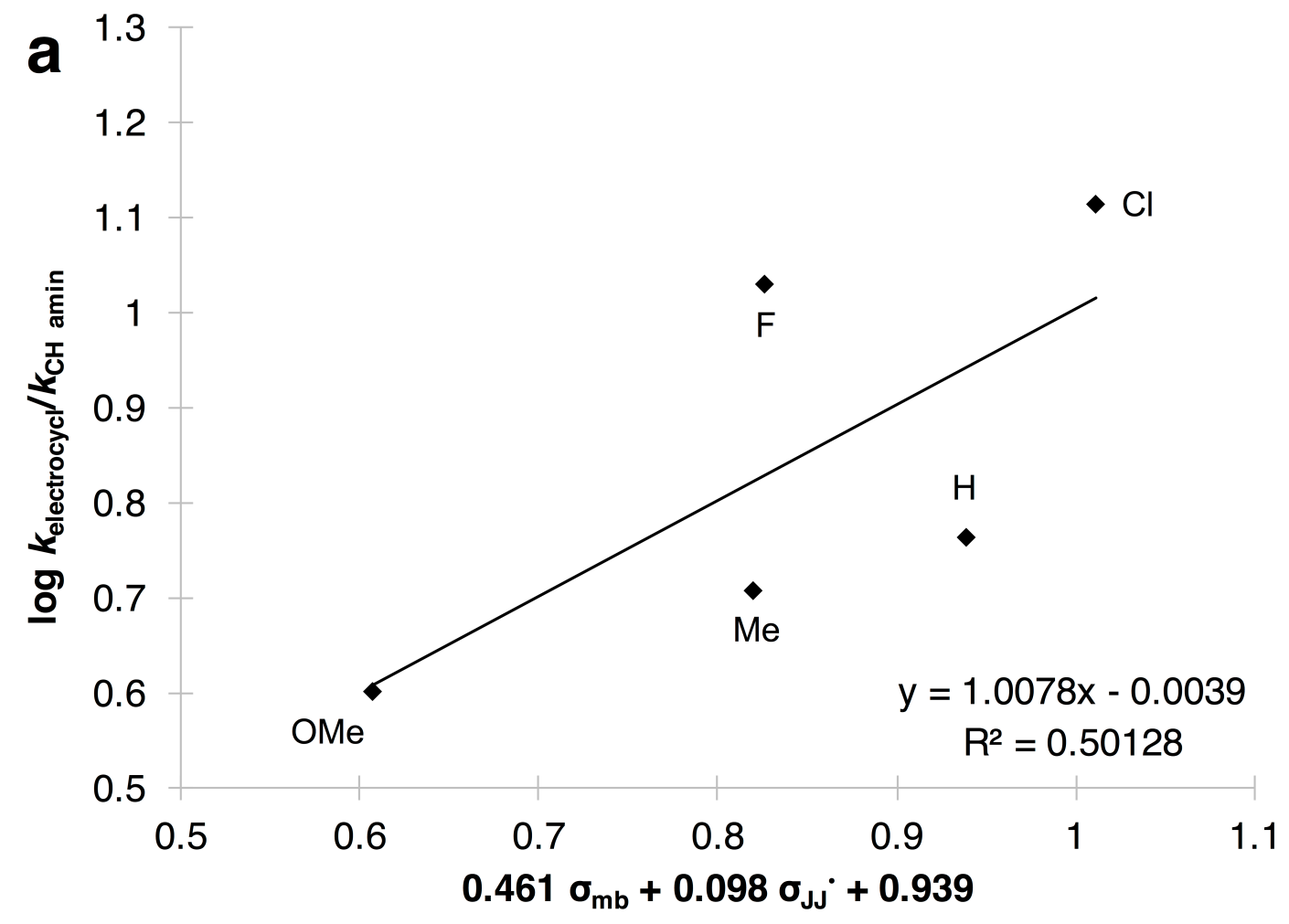

Figure s3. Correlation of heterocycle product ratios with Arnold's $\sigma_{\alpha}{ }^{\circ}$ values: substituent effect observed at the allylic $\mathrm{C}-\mathrm{H}$ reaction site.

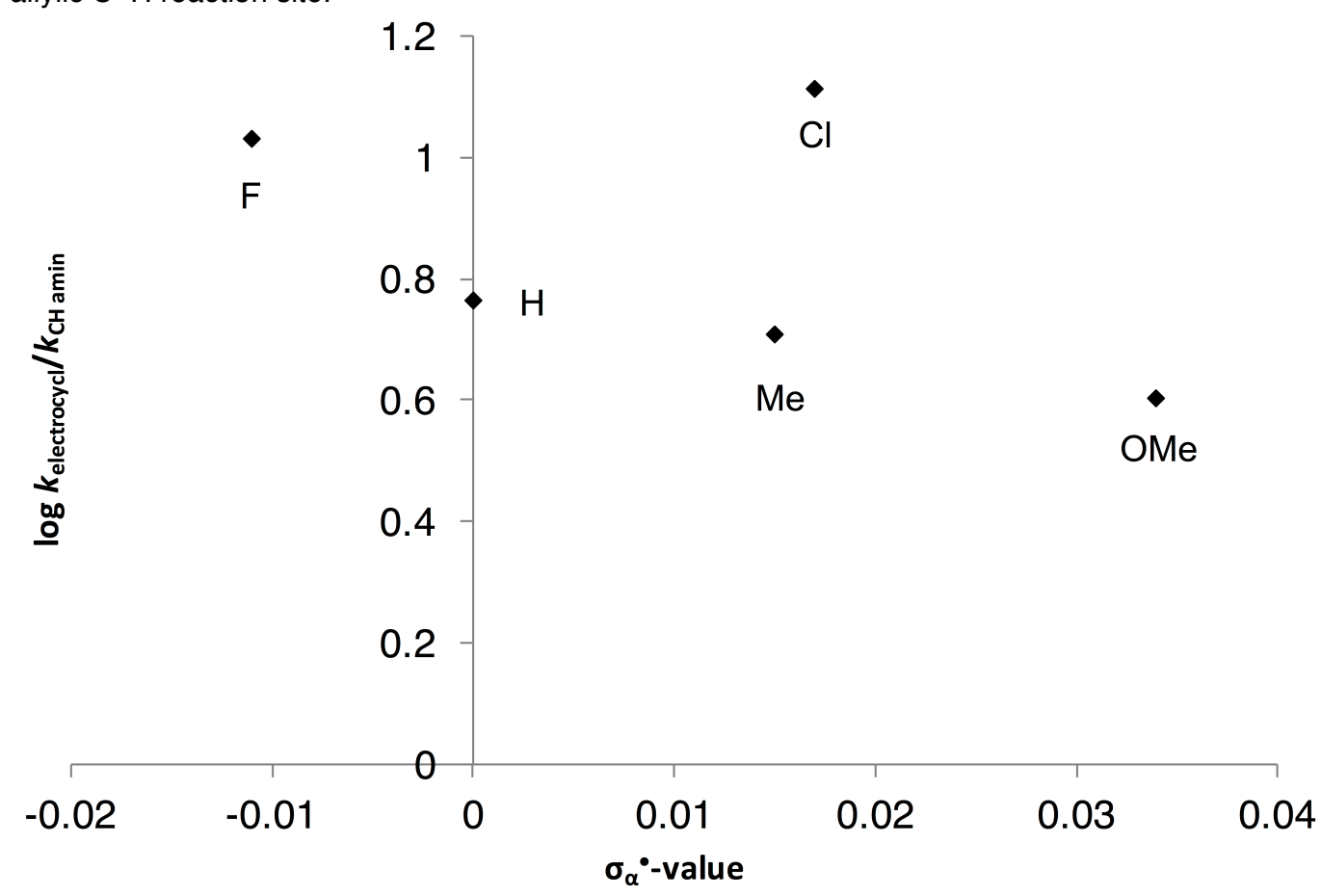


Figure s4. Correlation of heterocycle product ratios with Creary's $\sigma_{C}$ 'values: substituent effect observed at the allylic $\mathrm{C}-\mathrm{H}$ reaction site.

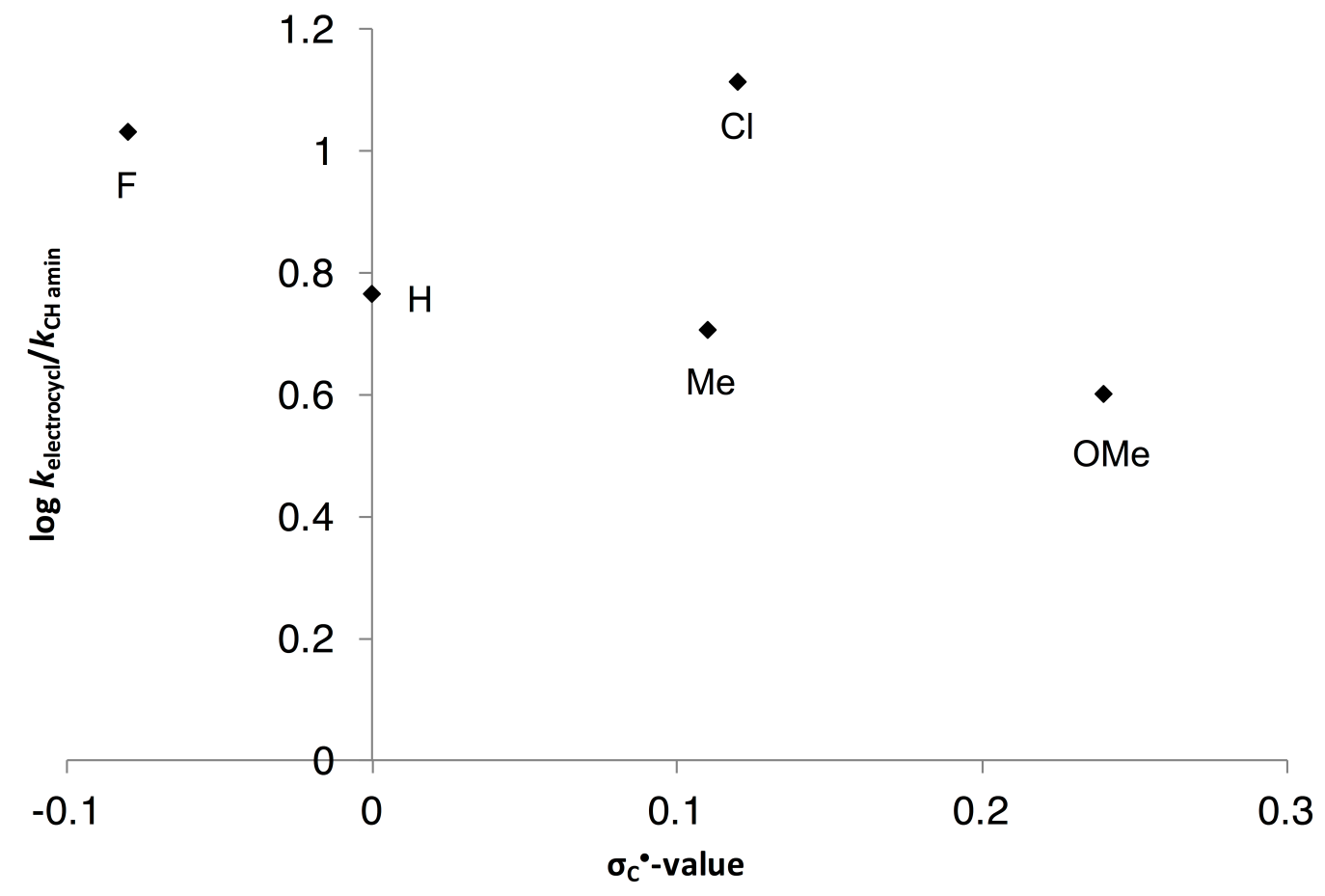

Figure s5. Correlation of heterocycle product ratios with Jackson's $\sigma_{j}$ values: substituent effect observed at the allylic $\mathrm{C}-\mathrm{H}$ reaction site.

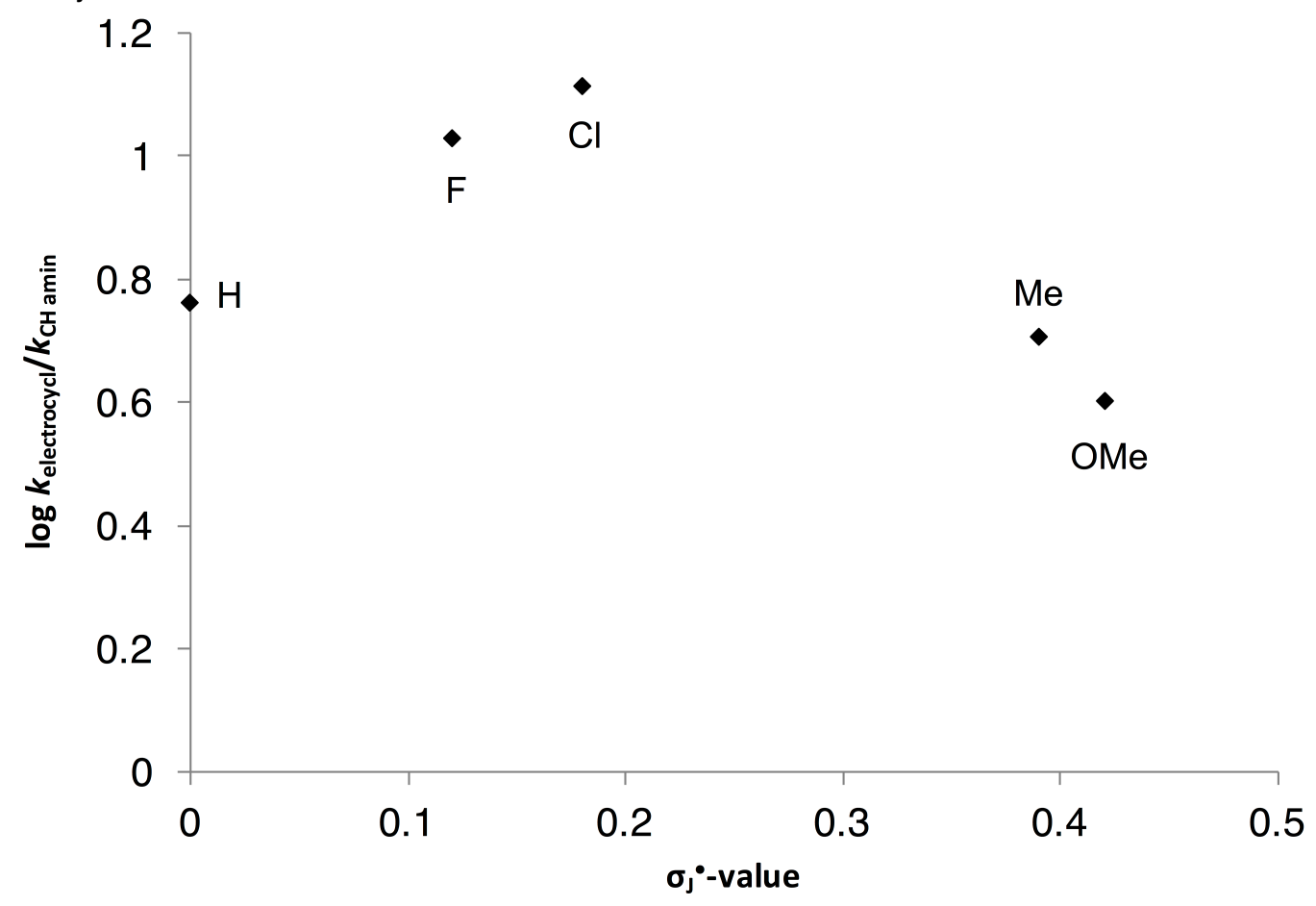




\section{B. Hammett study: effect of changing the electronic nature of the rhodium $\mathrm{N}$-aryl nitrene on the reaction} outcome.

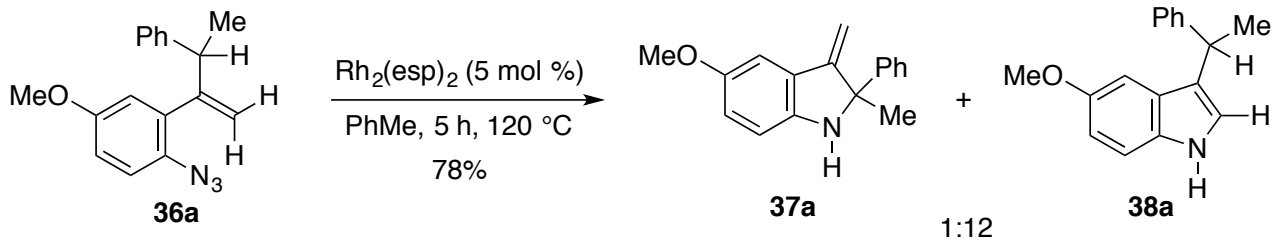

To a mixture of $0.0118 \mathrm{~g}$ of styryl azide $36 \mathrm{a}(0.0420 \mathrm{mmol})$ and $0.0016 \mathrm{~g}$ of $\mathrm{Rh}_{2}(\mathrm{esp})_{2}(0.0021 \mathrm{mmol})$ in a Schlenk tube was added $0.80 \mathrm{~mL}$ of toluene. The resulting mixture was heated to $120^{\circ} \mathrm{C}$. After $5 \mathrm{~h}$, the mixture was cooled to room temperature, diluted with $\mathrm{CH}_{2} \mathrm{Cl}_{2}$ and concentrated in vacuo to afford a mixture of 37a and 38a. The resulting residue was dissolved in $0.5 \mathrm{~mL}$ of $\mathrm{CDCl}_{3}$ and $7 \mu \mathrm{L}$ of dibromomethane $(0.1 \mathrm{mmol})$ was added. The area of the olefin $\mathrm{C}-\mathrm{H}$ peak of 37a was compared with benzylic $\mathrm{C}-\mathrm{H}$ peak of 38a to derive a ratio of $\mathbf{3 7 a : 3 8 a}(1: 12)$.<smiles>Cc1ccc(N)c(/C=C/c2ccccc2)c1</smiles>

$36 b$

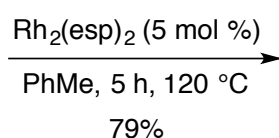

$79 \%$<smiles>C=C1c2cc(C)ccc2NC1(C)c1ccccc1</smiles>

$1: 7.2$<smiles>Cc1ccc2[nH]cc(C(C)c3ccccc3)c2c1</smiles>

(s38)

To a mixture of $0.0112 \mathrm{~g}$ of styryl azide $\mathbf{3 6} \mathbf{b}(0.0420 \mathrm{mmol})$ and $0.0016 \mathrm{~g}$ of $\mathrm{Rh}_{2}(\mathrm{esp})_{2}(0.0021 \mathrm{mmol})$ in a Schlenk tube was added $0.80 \mathrm{~mL}$ of toluene. The resulting mixture was heated to $120^{\circ} \mathrm{C}$. After $5 \mathrm{~h}$, the mixture was cooled to room temperature, diluted with $\mathrm{CH}_{2} \mathrm{Cl}_{2}$ and concentrated in vacuo to afford a mixture of $\mathbf{3 7} \mathbf{b}$ and $\mathbf{3 8 \mathbf { b }}$. The resulting residue was dissolved in $0.5 \mathrm{~mL}$ of $\mathrm{CDCl}_{3}$ and $7 \mu \mathrm{L}$ of dibromomethane $(0.1 \mathrm{mmol})$ was added. The area of the olefin $\mathrm{C}-\mathrm{H}$ peak of $\mathbf{3 7} \mathbf{b}$ was compared with benzylic $\mathrm{C}-\mathrm{H}$ peak of $\mathbf{3 8 b}$ to derive a ratio of $\mathbf{3 7 b} \mathbf{b} \mathbf{3 8 b}$ (1:7.2).
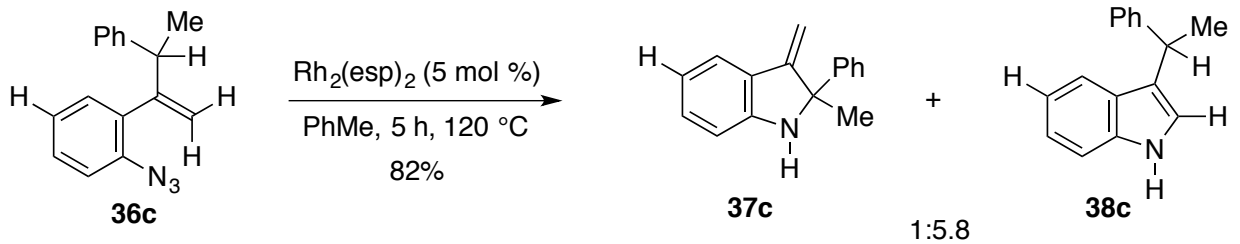

(s39)

To a mixture of $0.0119 \mathrm{~g}$ of styryl azide 36c $(0.0480 \mathrm{mmol})$ and $0.0018 \mathrm{~g}$ of $\mathrm{Rh}_{2}(\mathrm{esp})_{2}(0.0024 \mathrm{mmol})$ in a Schlenk tube was added $1.0 \mathrm{~mL}$ of toluene. The resulting mixture was heated to $120^{\circ} \mathrm{C}$. After $5 \mathrm{~h}$, the mixture was cooled to room temperature, diluted with $\mathrm{CH}_{2} \mathrm{Cl}_{2}$ and concentrated in vacuo to afford a mixture of 37c and 38c. The resulting residue was dissolved in $0.5 \mathrm{~mL}$ of $\mathrm{CDCl}_{3}$ and $7 \mu \mathrm{L}$ of dibromomethane $(0.1 \mathrm{mmol})$ was added. The area of the olefin $\mathrm{C}-\mathrm{H}$ peak of $37 \mathbf{c}$ was compared with benzylic $\mathrm{C}-\mathrm{H}$ peak of $38 \mathbf{c}$ to derive a ratio of $\mathbf{3 7} \mathbf{c}: \mathbf{3 8 c}(1: 5.8)$.
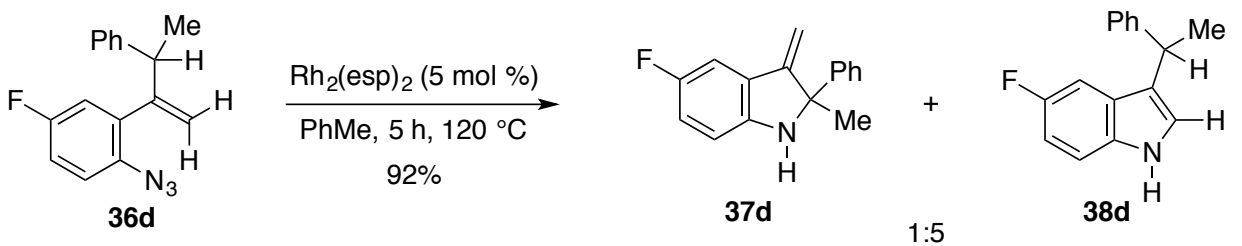

$(s 40)$

To a mixture of $0.0134 \mathrm{~g}$ of styryl azide $36 \mathrm{a}(0.0500 \mathrm{mmol})$ and $0.0019 \mathrm{~g}$ of $\mathrm{Rh}_{2}(\mathrm{esp})_{2}(0.0025 \mathrm{mmol})$ in a Schlenk tube was added $1.0 \mathrm{~mL}$ of toluene. The resulting mixture was heated to $120^{\circ} \mathrm{C}$. After $5 \mathrm{~h}$, the mixture was cooled to room temperature, diluted with $\mathrm{CH}_{2} \mathrm{Cl}_{2}$ and concentrated in vacuo to afford a mixture of $\mathbf{3 7 d}$ and $\mathbf{3 8 d}$. The resulting residue 
was dissolved in $0.5 \mathrm{~mL}$ of $\mathrm{CDCl}_{3}$ and $7 \mu \mathrm{L}$ of dibromomethane $(0.1 \mathrm{mmol})$ was added. The area of the olefin $\mathrm{C}-\mathrm{H}$ peak of $\mathbf{3 7 d}$ was compared with benzylic $\mathrm{C}-\mathrm{H}$ peak of $\mathbf{3 8 d}$ to derive a ratio of $\mathbf{3 7 d}: \mathbf{3 8 d}(1: 5)$.
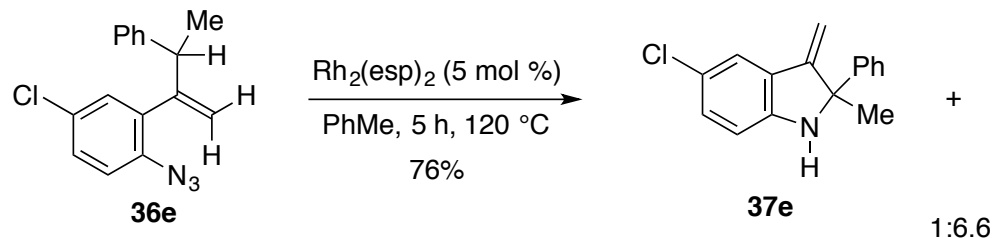<smiles>CC(c1ccccc1)c1c[nH]c2ccc(Cl)cc12</smiles>

(s41)

To a mixture of $0.0135 \mathrm{~g}$ of styryl azide $36 \mathrm{e}(0.0480 \mathrm{mmol})$ and $0.0018 \mathrm{~g}$ of $\mathrm{Rh}_{2}(\mathrm{esp})_{2}(0.0024 \mathrm{mmol})$ in a Schlenk tube was added $1.0 \mathrm{~mL}$ of toluene. The resulting mixture was heated to $120{ }^{\circ} \mathrm{C}$. After $5 \mathrm{~h}$, the mixture was cooled to room temperature, diluted with $\mathrm{CH}_{2} \mathrm{Cl}_{2}$ and concentrated in vacuo to afford a mixture of $37 \mathbf{e}$ and $\mathbf{3 8 e}$. The resulting residue was dissolved in $0.5 \mathrm{~mL}$ of $\mathrm{CDCl}_{3}$ and $7 \mu \mathrm{L}$ of dibromomethane $(0.1 \mathrm{mmol})$ was added. The area of the olefin $\mathrm{C}-\mathrm{H}$ peak of $37 \mathbf{e}$ was compared with benzylic $\mathrm{C}-\mathrm{H}$ peak of $38 \mathbf{e}$ to derive a ratio of $\mathbf{3 7 e}: \mathbf{3 8 e} \mathbf{e}(1: 6.6)$.

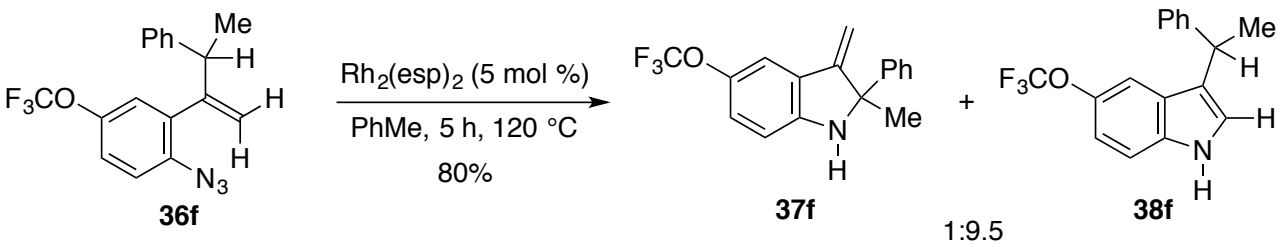

To a mixture of $0.0177 \mathrm{~g}$ of styryl azide $36 \mathrm{f}(0.0530 \mathrm{mmol})$ and $0.0020 \mathrm{~g}$ of $\mathrm{Rh}_{2}(\mathrm{esp})_{2}(0.0026 \mathrm{mmol})$ in a Schlenk tube was added $1.1 \mathrm{~mL}$ of toluene. The resulting mixture was heated to $120^{\circ} \mathrm{C}$. After $5 \mathrm{~h}$, the mixture was cooled to room temperature, diluted with $\mathrm{CH}_{2} \mathrm{Cl}_{2}$ and concentrated in vacuo to afford a mixture of $\mathbf{3 7 f}$ and $\mathbf{3 8 f}$. The resulting solid was dissolved in $0.5 \mathrm{~mL}$ of $\mathrm{CDCl}_{3}$ and $7 \mu \mathrm{L}$ of dibromomethane $(0.1 \mathrm{mmol})$ was added. The area of the olefin $\mathrm{C}-\mathrm{H}$ peak of $\mathbf{3 7 f}$ was compared with benzylic $\mathrm{C}-\mathrm{H}$ peak of $\mathbf{3 8 f}$ to derive a ratio of $\mathbf{3 7 f : 3 8 f}$ (1:9.5).

Table s4. Effect of changing the electronic nature of the rhodium $\mathrm{N}$-aryl nitrene on the reaction outcome.

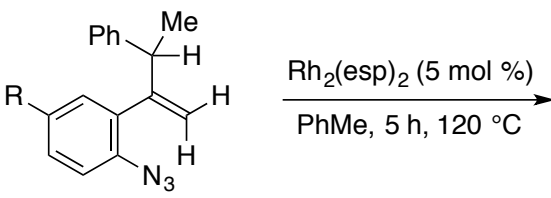

36

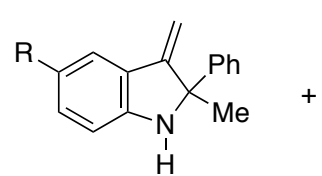

37<smiles>[R]c1ccc2[nH]cc(C(C)c3ccccc3)c2c1</smiles>

38

\begin{tabular}{|c|c|c|c|c|c|c|c|c|c|c|}
\hline entry & $\#$ & $\mathrm{R}$ & $\sigma_{\text {para }}{ }^{a}$ & $\sigma_{m b}^{b}$ & $\sigma_{J \jmath} \cdot b$ & $\sigma_{a}{ }^{c}$ & $\sigma_{C} \cdot d$ & $\sigma_{J}{ }^{e}$ & $37: 38$ & yield, $\%^{\mathrm{c}}$ \\
\hline 1 & $a$ & $\mathrm{OMe}$ & -0.27 & -0.77 & 0.23 & 0.034 & 0.24 & 0.42 & $1: 12$ & 78 \\
\hline 2 & $b$ & $\mathrm{Me}$ & -0.17 & -0.29 & 0.15 & 0.015 & 0.11 & 0.39 & $1: 7.2$ & 79 \\
\hline 3 & c & $\mathrm{H}$ & 0 & 0 & 0 & 0 & 0 & 0 & 1.5 .8 & 82 \\
\hline 4 & $d$ & $F$ & 0.06 & -0.24 & -0.02 & -0.011 & -0.08 & 0.12 & $1: 5$ & 92 \\
\hline 5 & e & $\mathrm{Cl}$ & 0.23 & 0.11 & 0.22 & 0.017 & 0.12 & 0.18 & $1: 6.6$ & 76 \\
\hline 6 & $f$ & $\mathrm{OCF}_{3}$ & 0.35 & $\ldots$ & $\ldots$ & $\ldots$ & $\ldots$ & $\ldots$ & $1: 9.5$ & 80 \\
\hline
\end{tabular}

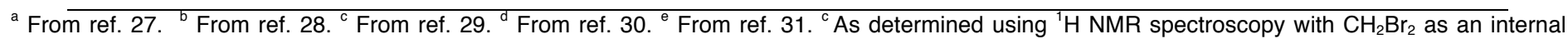
standard. 
Figure s6. Effect of changing the electronic environment of the rhodium $N$-aryl nitrene: correlation of heterocycle product ratios with Hammett $\sigma_{\text {para }}$ values.

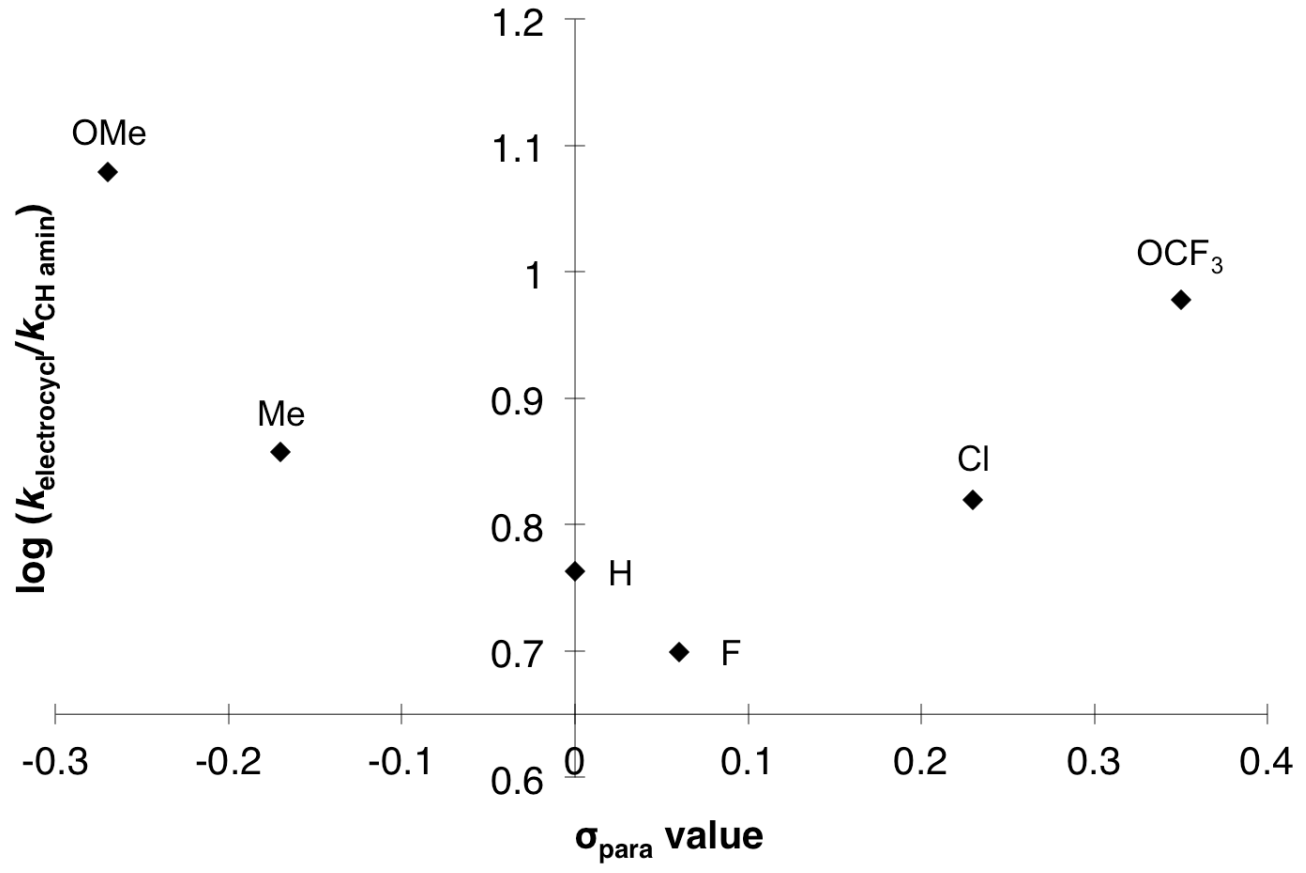

Figure s7. Correlation of heterocycle product ratios with Jiang and $\mathrm{Ji} \sigma_{\mathrm{mb}}$ and $\sigma_{J j}$ 'values: substituent effect observed on the aryl azide.

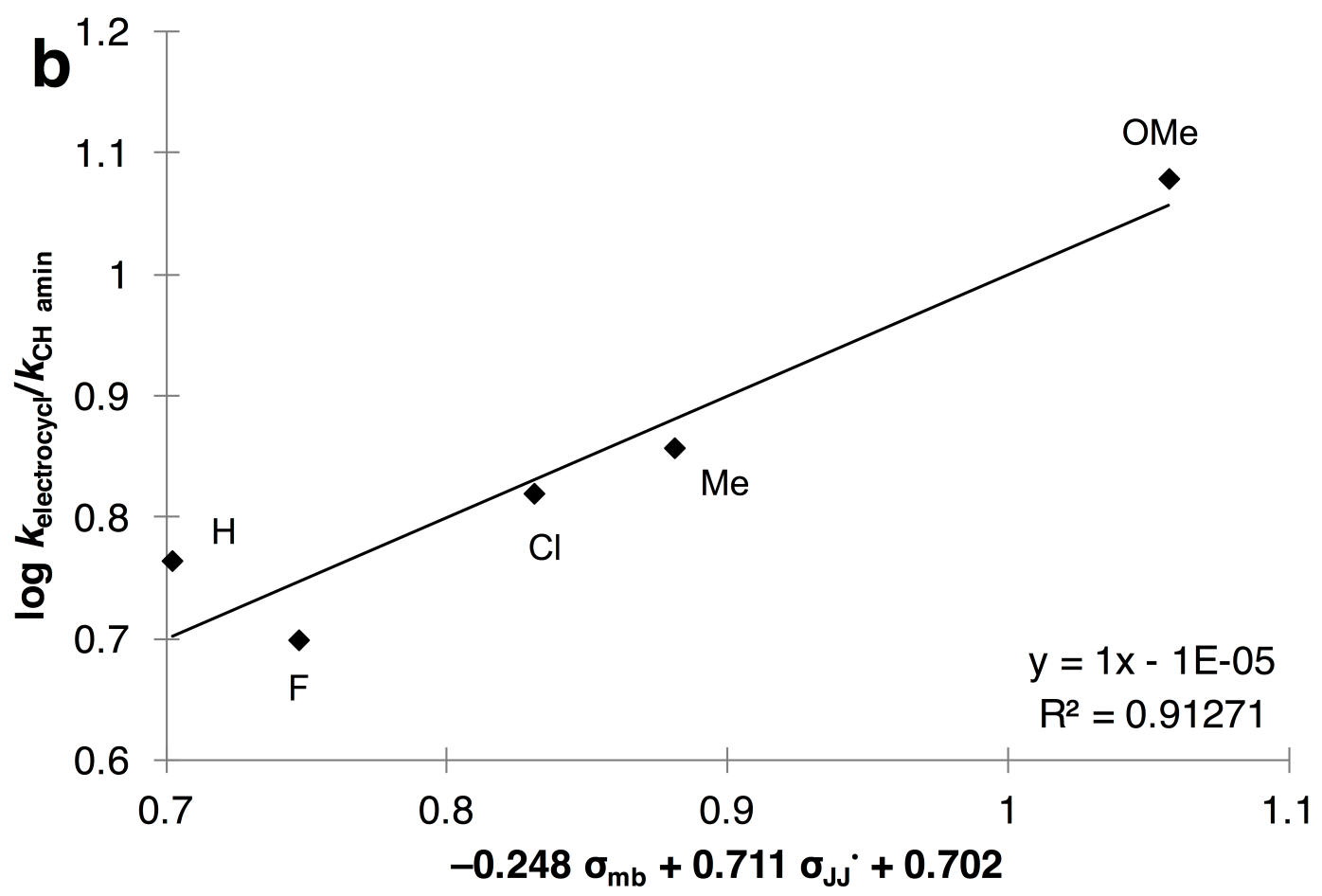


Figure s8. Correlation of heterocycle product ratios with Arnold's $\sigma_{a}{ }^{\circledR}$ values: substituent effect observed on the aryl azide.

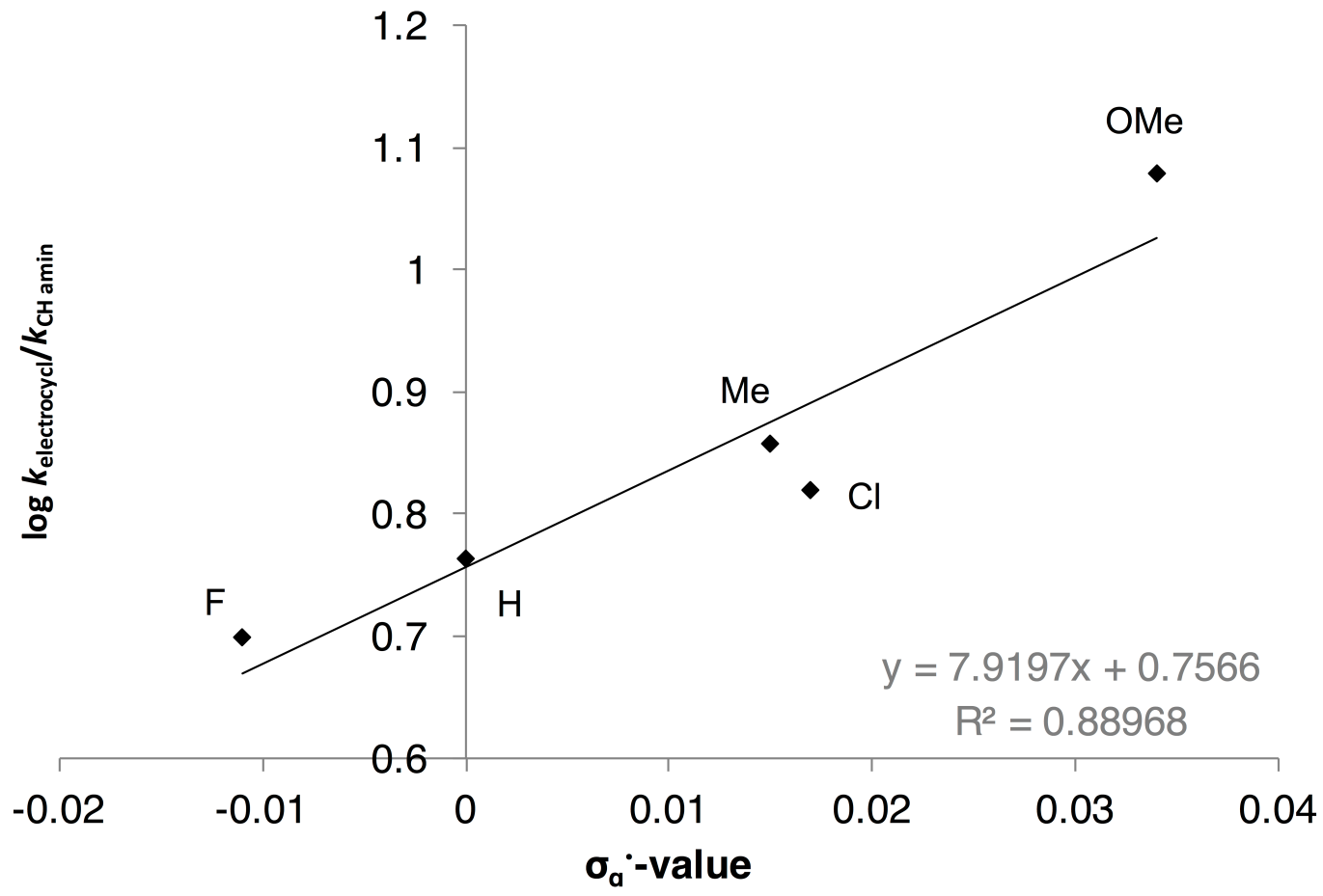

Figure s9. Correlation of heterocycle product ratios with Creary's $\sigma_{C}{ }^{\circ}$ values: substituent effect observed on the aryl azide.

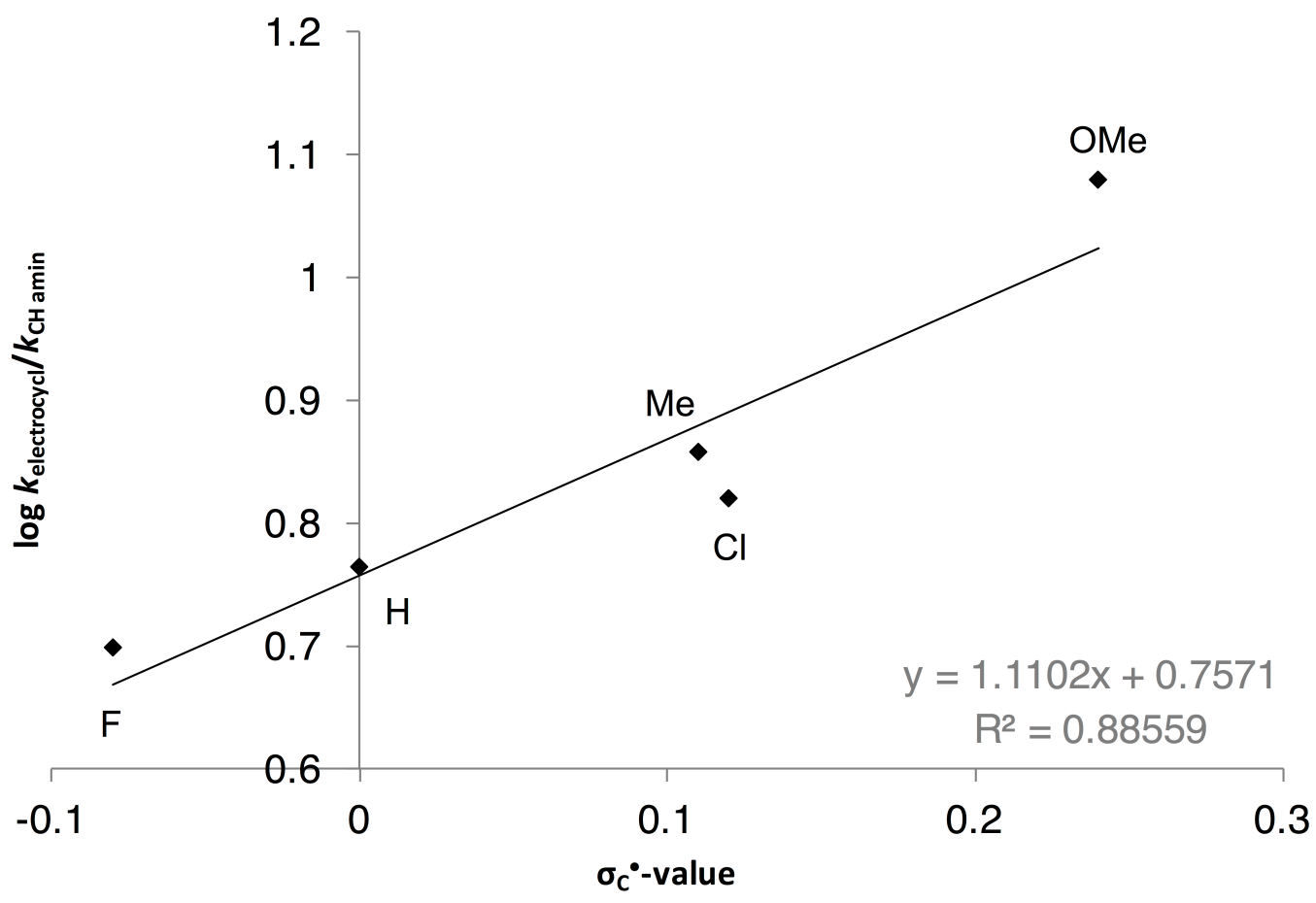


Figure s10. Correlation of heterocycle product ratios with Jackson's $\sigma_{j}$ values: substituent effect observed on the aryl azide.

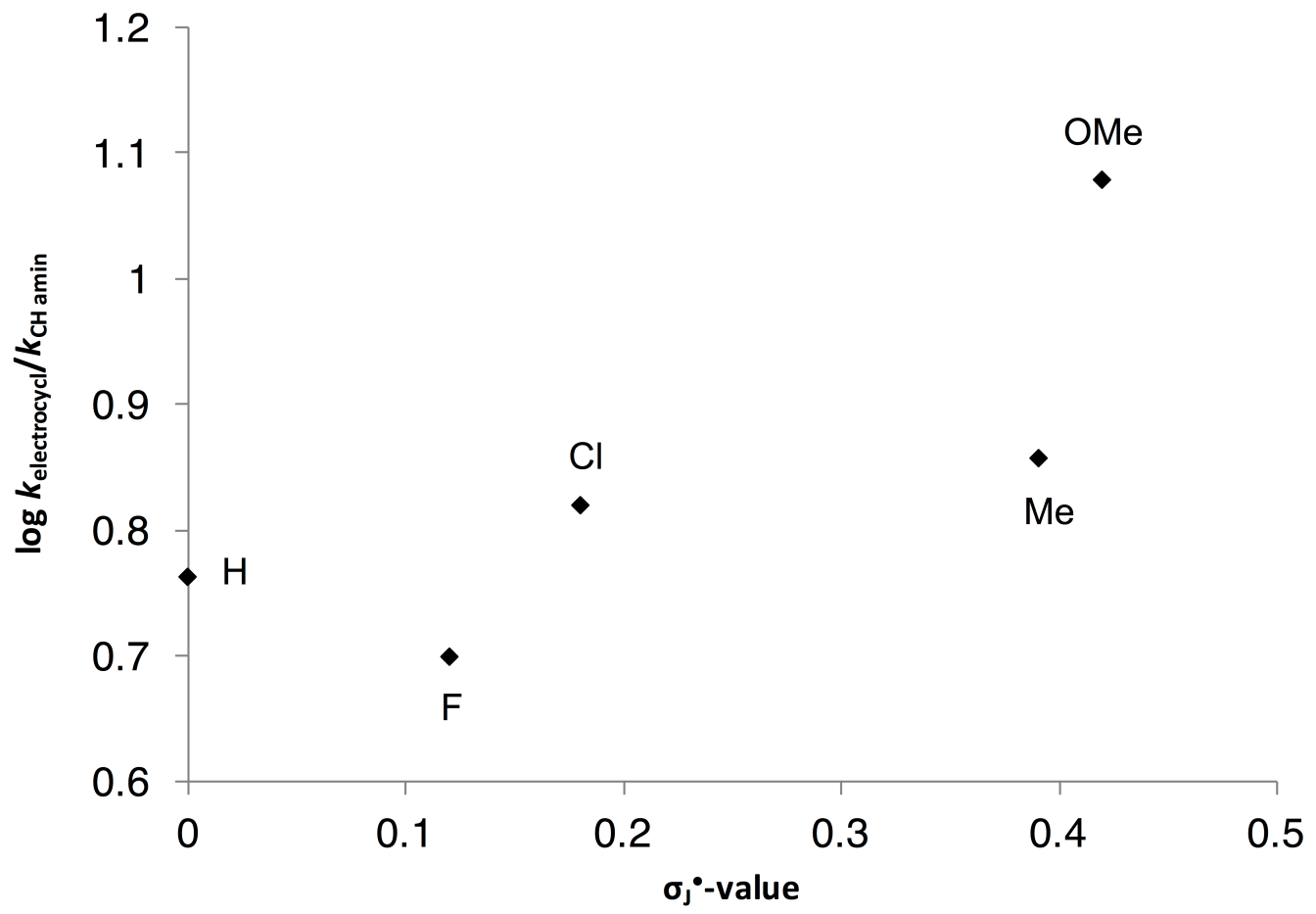

C. Relationship between isotopolog identity and ratio of $2 \mathrm{H}$-indole $33 \mathrm{~b}$ and indole $34 \mathrm{~b}$ using $\mathrm{Rh}_{2}(\mathrm{esp})_{2}$ as the catalyst.

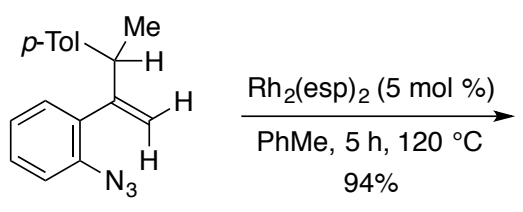

33b<smiles>C=C1c2ccccc2NC1(C)C(=O)[O-]</smiles>

$34 b$<smiles>CC(C)(C)c1c[nH]c2ccccc12</smiles>

$35 b$

To a mixture of $0.0225 \mathrm{~g}$ of styryl azide $33 \mathrm{~b}(0.0850 \mathrm{mmol})$ and $0.0032 \mathrm{~g}$ of $\mathrm{Rh}_{2}(\mathrm{esp})_{2}(0.0042 \mathrm{mmol})$ in a Schlenk tube was added $1.7 \mathrm{~mL}$ of toluene. The resulting mixture was heated to $120{ }^{\circ} \mathrm{C}$. After $5 \mathrm{~h}$, the mixture was cooled to room temperature, diluted with $\mathrm{CH}_{2} \mathrm{Cl}_{2}$ and concentrated in vacuo to afford a mixture of $\mathbf{3 4 \mathbf { b }}$ and $\mathbf{3 5 \mathbf { b }}$. The resulting residue was dissolved in $0.5 \mathrm{~mL}$ of $\mathrm{CDCl}_{3}$ and $7 \mu \mathrm{L}$ of dibromomethane $(0.1 \mathrm{mmol})$ was added. The area of the olefin $\mathrm{C}-\mathrm{H}$ peak of $\mathbf{3 4 b}$ was compared with benzylic $\mathrm{C}-\mathrm{H}$ peak of $\mathbf{3 5 b}$ to derive a ratio of $\mathbf{3 4 \mathbf { b } : 3 5 \mathbf { b }}$ (1:5.1). 


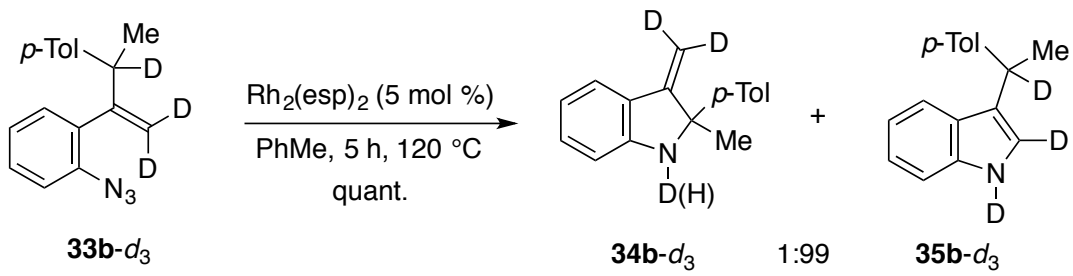

To a mixture of $0.0180 \mathrm{~g}$ of styryl azide $33 \mathrm{~b}-d_{3}(0.0670 \mathrm{mmol})$ and $0.0025 \mathrm{~g} \mathrm{of} \mathrm{Rh}_{2}(\mathrm{esp})_{2}(0.0033 \mathrm{mmol})$ in a Schlenk tube was added $1.3 \mathrm{~mL}$ of toluene. The resulting mixture was heated to $120{ }^{\circ} \mathrm{C}$. After $5 \mathrm{~h}$, the mixture was cooled to

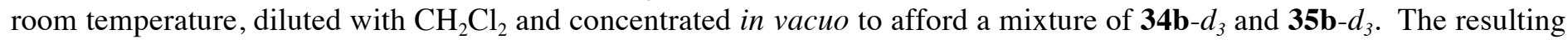
solid was dissolved in $0.5 \mathrm{~mL}$ of $\mathrm{CDCl}_{3}$ and $7 \mu \mathrm{L}$ of dibromomethane $(0.1 \mathrm{mmol})$ was added. The area of the methyl $\mathrm{CH}_{3}$

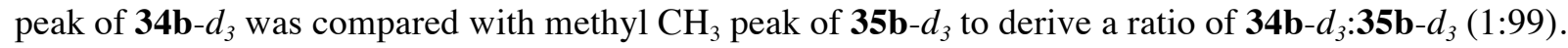

\section{the catalyst.}

\section{Relationship between isotopolog identity and ratio of $2 H$-indole $45-d_{0}$ and indole $45-d_{2}$ using $\operatorname{Rh}_{2}(\operatorname{esp})_{2}$ as}

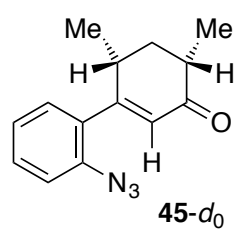
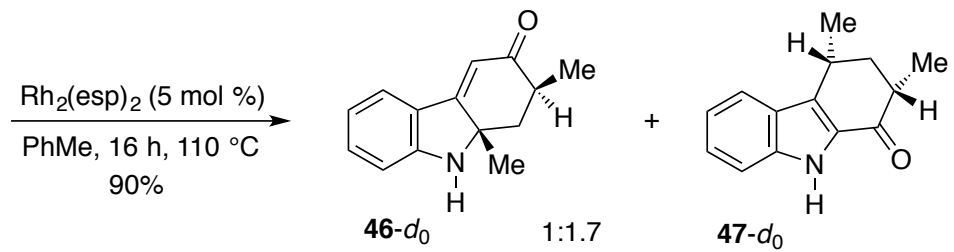

(s45)

To a mixture of $0.0193 \mathrm{~g}$ of styryl azide $45-d_{0}(0.0800 \mathrm{mmol})$ and $0.0030 \mathrm{~g}$ of $\mathrm{Rh}_{2}(\mathrm{esp})_{2}(0.0040 \mathrm{mmol})$ in a Schlenk tube was added $1.7 \mathrm{~mL}$ of toluene. The resulting mixture was heated to $110^{\circ} \mathrm{C}$. After $16 \mathrm{~h}$, the mixture was cooled to room temperature, diluted with $\mathrm{CH}_{2} \mathrm{Cl}_{2}$ and concentrated in vacuo to afford a mixture of 46- $d_{0}$ and $47-d_{0}$. The resulting residue was dissolved in $0.5 \mathrm{~mL}$ of $\mathrm{CDCl}_{3}$ and $7 \mu \mathrm{L}$ of dibromomethane $(0.1 \mathrm{mmol})$ was added. The area of the $-\mathrm{CH}_{3}$ peak of 46$d_{0}$ at $1.49 \mathrm{ppm}$ was compared with $-\mathrm{CH}_{3}$ peak of $47-d_{0}$ at $1.61 \mathrm{ppm}$ to derive a ratio of $46-d_{0}: 47-d_{0}(1: 1.7)$. A $5: 1$ diastereomeric mixture was observed for compound 46- $d_{0}$ and 6:1 diastereomeric mixture was observed for compound 47$d_{0}$.
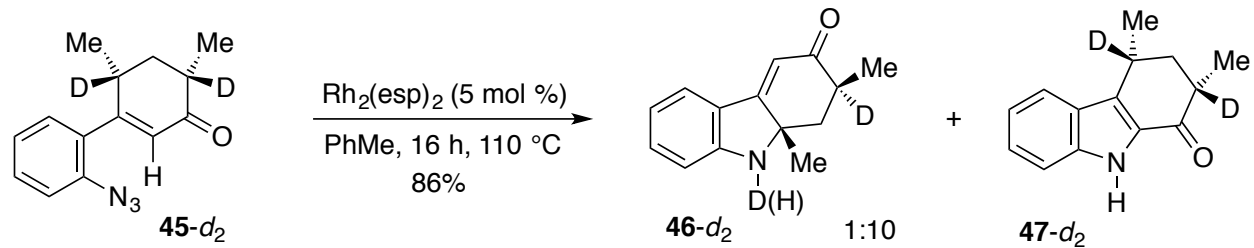

(s46)

To a mixture of $0.0130 \mathrm{~g}$ of styryl azide $45-d_{2}(0.0530 \mathrm{mmol})$ and $0.0032 \mathrm{~g}$ of $\mathrm{Rh}_{2}(\mathrm{esp})_{2}(0.0026 \mathrm{mmol})$ in a Schlenk tube was added $1.0 \mathrm{~mL}$ of toluene. The resulting mixture was heated to $110^{\circ} \mathrm{C}$. After $16 \mathrm{~h}$, the mixture was cooled to room temperature, diluted with $\mathrm{CH}_{2} \mathrm{Cl}_{2}$ and concentrated in vacuo to afford a mixture of 46- $d_{2}$ and $47-d_{2}$. The resulting solid was dissolved in $0.5 \mathrm{~mL}$ of $\mathrm{CDCl}_{3}$ and $7 \mu \mathrm{L}$ of dibromomethane $(0.1 \mathrm{mmol})$ was added. The area of the $-\mathrm{CH}_{3}$ peak of 46$d_{2}$ at $1.49 \mathrm{ppm}$ was compared with $-\mathrm{CH}_{3}$ peak of $47-d_{2}$ at $1.61 \mathrm{ppm}$ to derive a ratio of $\mathbf{4 6}-d_{2}: 47-d_{2}(1: 10)$. A $3: 1$ diastereomeric mixture was observed for compound 46- $d_{2}$ and 10:1 diastereomeric mixture was observed for compound 47- $d_{2}$. 
E. Relationship between isotopolog identity and ratio of $2 \mathrm{H}$-indole $45-d_{0}$ and indole $45-d_{2}$ using $\mathrm{Fe}(\mathrm{OEP}) \mathrm{Cl}$ as the catalyst.<smiles>C[C@H]1C[C@H](C)[C@H](c2ccccc2N)C=C1C=O</smiles>

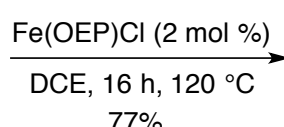

$77 \%$

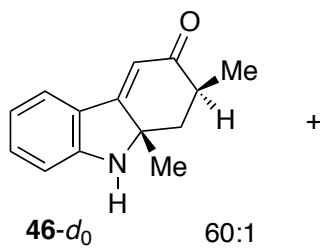<smiles>C[C@H]1C[C@H](C)c2c([nH]c3ccccc23)C1=O</smiles>

To a mixture of $0.0115 \mathrm{~g}$ of styryl azide $45-d_{0}(0.0470 \mathrm{mmol})$ and $0.0006 \mathrm{~g}$ of $\mathrm{Fe}(\mathrm{OEP}) \mathrm{Cl}(0.0009 \mathrm{mmol})$ in a Schlenk tube was added $1.7 \mathrm{~mL}$ of toluene. The resulting mixture was heated to $120^{\circ} \mathrm{C}$. After $16 \mathrm{~h}$, the mixture was cooled to room temperature, diluted with $\mathrm{CH}_{2} \mathrm{Cl}_{2}$ and concentrated in vacuo to afford a mixture of 46- $d_{0}$ and $47-d_{0}$. The resulting residue was dissolved in $0.5 \mathrm{~mL}$ of $\mathrm{CDCl}_{3}$ and $7 \mu \mathrm{L}$ of dibromomethane $(0.1 \mathrm{mmol})$ was added. The area of the $-\mathrm{CH}_{3}$ peak of 46- $d_{0}$ at $1.49 \mathrm{ppm}$ was compared with $-\mathrm{CH}_{3}$ peak of $47-d_{0}$ at $1.61 \mathrm{ppm}$ to derive a ratio of $46-d_{0}: 47-d_{0}(60: 1)$. A 2.4:1 diastereomeric mixture was observed for compound $\mathbf{4 6}-d_{0}$.<smiles>[2H]C1(C)C[C@]([2H])(C)C(C(=O)O)=C[C@@]1([2H])c1ccccc1N</smiles>

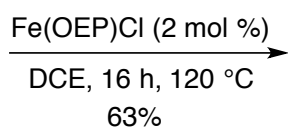<smiles>[2H]N1c2ccccc2C2=CC(=O)C(C)(C)CC21C</smiles><smiles>[2H][C@]1(C)C[C@@]([2H])(C)c2c([nH]c3ccccc23)C1=O</smiles>

(s48)

To a mixture of $0.0115 \mathrm{~g}$ of styryl azide $45-d_{2}(0.0470 \mathrm{mmol})$ and $0.0006 \mathrm{~g}$ of $\mathrm{Fe}(\mathrm{OEP}) \mathrm{Cl}(0.0009 \mathrm{mmol})$ in a Schlenk tube was added $0.8 \mathrm{~mL}$ of toluene. The resulting mixture was heated to $120^{\circ} \mathrm{C}$. After $16 \mathrm{~h}$, the mixture was cooled to room temperature, diluted with $\mathrm{CH}_{2} \mathrm{Cl}_{2}$ and concentrated in vacuo to afford a mixture of 46- $d_{2}$ and 47- $d_{2}$. The resulting solid was dissolved in $0.5 \mathrm{~mL}$ of $\mathrm{CDCl}_{3}$ and $7 \mu \mathrm{L}$ of dibromomethane $(0.1 \mathrm{mmol})$ was added. The area of the $-\mathrm{CH}_{3}$ peak of 46- $d_{2}$ at $1.49 \mathrm{ppm}$ was compared with $-\mathrm{CH}_{3}$ peak of $47-d_{2}$ at $1.61 \mathrm{ppm}$ to derive a ratio of $46-d_{2}: 47-d_{2}(2: 1)$. A $1: 1$ diastereomeric mixture was observed for compound 46- $d_{2}$ and $>20: 1$ diastereomeric mixture was observed for compound 47- $d_{2}$.

\section{Mechanistic Study for Fe(OEP)Cl-Catalyzed sp $^{3}$ C-H Bond Amination.}

A. Hammett study: effect of changing the electronic nature of the iron $N$-aryl nitrene on the reaction outcome.<smiles>COC(=O)C1=C(c2cccc([N+]#N)c2)CCCC1</smiles>

$16 a$<smiles>COC(=O)C1=C(c2cc(OC)ccc2N)CCCC1</smiles>

16b

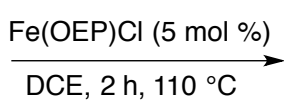

DCE, $2 \mathrm{~h}, 110^{\circ} \mathrm{C}$

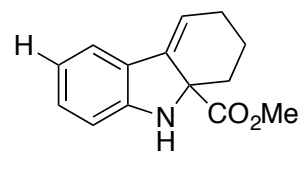

$53 a$<smiles>COc1ccc2c(c1)C1=CCCCC1(C(C)=O)N2</smiles>

$53 b$

To a mixture of $0.0100 \mathrm{~g}$ of styryl azide $16 \mathbf{a}(0.038 \mathrm{mmol}), 0.0145 \mathrm{~g}$ of styryl azide $16 \mathbf{b}(0.050 \mathrm{mmol})$ and $0.0027 \mathrm{~g}$ of $\mathrm{Fe}(\mathrm{OEP}) \mathrm{Cl}(0.0040 \mathrm{mmol})$ in a Schlenk tube was added $1.0 \mathrm{~mL}$ of 1,2-dichloroethane. The resulting mixture was heated to $110{ }^{\circ} \mathrm{C}$. After $2 \mathrm{~h}$, the mixture was cooled to room temperature, diluted with ethyl acetate. The solution was filtered through a plug of celite and concentrated in vacuo to afford a mixture of $\mathbf{1 6 a}, \mathbf{1 6 b}, \mathbf{5 3 \mathbf { a }}$ and $\mathbf{5 3 \mathbf { b }}$. The resulting residue was dissolved in $0.5 \mathrm{~mL}$ of $\mathrm{CDCl}_{3}$ and $7 \mu \mathrm{L}$ of dibromomethane $(0.1 \mathrm{mmol})$ was added. Analysis of the resulting residue 
using ${ }^{1} \mathrm{H}$ NMR spectroscopy with $\mathrm{CH}_{2} \mathrm{Br}_{2}$ as the internal standard revealed that 0.032 mmol of styryl azide $\mathbf{1 6 a}, 0.023$ mmol of styryl azide 16c left in the reaction mixture.

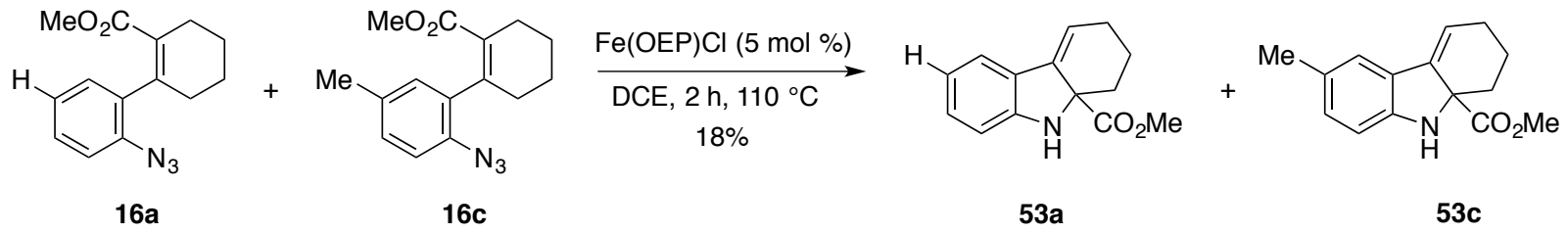

To a mixture of $0.0139 \mathrm{~g}$ of styryl azide $16 \mathbf{a}(0.054 \mathrm{mmol}), 0.0144 \mathrm{~g}$ of styryl azide $16 \mathrm{c}(0.054 \mathrm{mmol})$ and $0.0033 \mathrm{~g}$ of $\mathrm{Fe}(\mathrm{OEP}) \mathrm{Cl}(0.0054 \mathrm{mmol})$ in a Schlenk tube was added $1.0 \mathrm{~mL}$ of 1,2-dichloroethane. The resulting mixture was heated to $110{ }^{\circ} \mathrm{C}$. After $2 \mathrm{~h}$, the mixture was cooled to room temperature, diluted with ethyl acetate. The solution was filtered through a plug of celite and concentrated in vacuo to afford a mixture of $\mathbf{1 6 a}, \mathbf{1 6 c}, \mathbf{5 3 \mathbf { a }}$ and $\mathbf{5 3 \mathbf { c }}$. The resulting residue was dissolved in $0.5 \mathrm{~mL}$ of $\mathrm{CDCl}_{3}$ and $7 \mu \mathrm{L}$ of dibromomethane $(0.1 \mathrm{mmol})$ was added. Analysis of the resulting residue using ${ }^{1} \mathrm{H}$ NMR spectroscopy with $\mathrm{CH}_{2} \mathrm{Br}_{2}$ as the internal standard revealed that $0.040 \mathrm{mmol}$ of styryl azide 16a, 0.017 mmol of styryl azide $\mathbf{1 6 c}$ left in the reaction mixture.<smiles></smiles>

$16 a$<smiles>COC(=O)C1=C(c2cc(F)ccc2N)CCCC1</smiles>

$16 d$

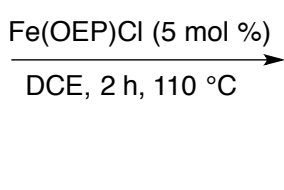

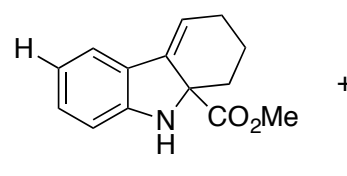

$53 a$<smiles>COC(=O)C12CCCC=C1c1cc(F)ccc1N2</smiles>

53d

To a mixture of $0.0135 \mathrm{~g}$ of styryl azide $16 \mathrm{a}(0.052 \mathrm{mmol}), 0.0144 \mathrm{~g}$ of styryl azide $16 \mathbf{d}(0.052 \mathrm{mmol})$ and $0.0032 \mathrm{~g}$ of $\mathrm{Fe}(\mathrm{OEP}) \mathrm{Cl}(0.0052 \mathrm{mmol})$ in a Schlenk tube was added $1.0 \mathrm{~mL}$ of 1,2-dichloroethane. The resulting mixture was heated to $110{ }^{\circ} \mathrm{C}$. After $2 \mathrm{~h}$, the mixture was cooled to room temperature, diluted with ethyl acetate. The solution was filtered through a plug of celite and concentrated in vacuo to afford a mixture of 16a, 16d, 53a and 53d. The resulting residue was dissolved in $0.5 \mathrm{~mL}$ of $\mathrm{CDCl}_{3}$ and $7 \mu \mathrm{L}$ of dibromomethane $(0.1 \mathrm{mmol})$ was added. Analysis of the resulting residue using ${ }^{1} \mathrm{H}$ NMR spectroscopy with $\mathrm{CH}_{2} \mathrm{Br}_{2}$ as the internal standard revealed that $0.021 \mathrm{mmol}$ of styryl azide 16a, 0.006 mmol of styryl azide 16d left in the reaction mixture.<smiles></smiles>

$16 a$<smiles>COC(=O)C1=C(c2cc(Cl)ccc2N)CCCC1</smiles>

$16 e$

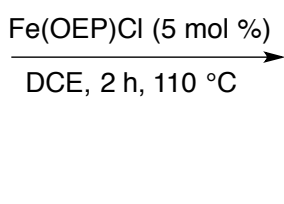

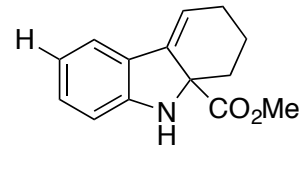

$53 a$

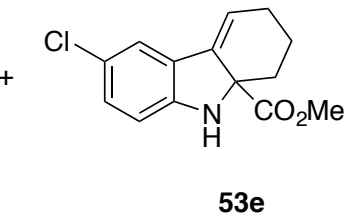

$53 e$

To a mixture of $0.0115 \mathrm{~g}$ of styryl azide $16 \mathrm{a}(0.045 \mathrm{mmol}), 0.0131 \mathrm{~g}$ of styryl azide $16 \mathrm{e}(0.045 \mathrm{mmol})$ and $0.0028 \mathrm{~g}$ of $\mathrm{Fe}(\mathrm{OEP}) \mathrm{Cl}(0.0045 \mathrm{mmol})$ in a Schlenk tube was added $1.0 \mathrm{~mL}$ of 1,2-dichloroethane. The resulting mixture was heated to $110^{\circ} \mathrm{C}$. After $2 \mathrm{~h}$, the mixture was cooled to room temperature, diluted with ethyl acetate. The solution was filtered through a plug of celite and concentrated in vacuo to afford a mixture of $\mathbf{1 6 a}, \mathbf{1 6 e}, \mathbf{5 3 \mathbf { a }}$ and $53 \mathbf{e}$. The resulting residue was dissolved in $0.5 \mathrm{~mL}$ of $\mathrm{CDCl}_{3}$ and $7 \mu \mathrm{L}$ of dibromomethane $(0.1 \mathrm{mmol})$ was added. Analysis of the resulting residue using ${ }^{1} \mathrm{H}$ NMR spectroscopy with $\mathrm{CH}_{2} \mathrm{Br}_{2}$ as the internal standard revealed that $0.025 \mathrm{mmol}$ of styryl azide 16a, 0.010 mmol of styryl azide 16e left in the reaction mixture. 
Table s5. Effect of changing the electronic environment on the reactivity of the iron $\mathrm{N}$-aryl nitrene.

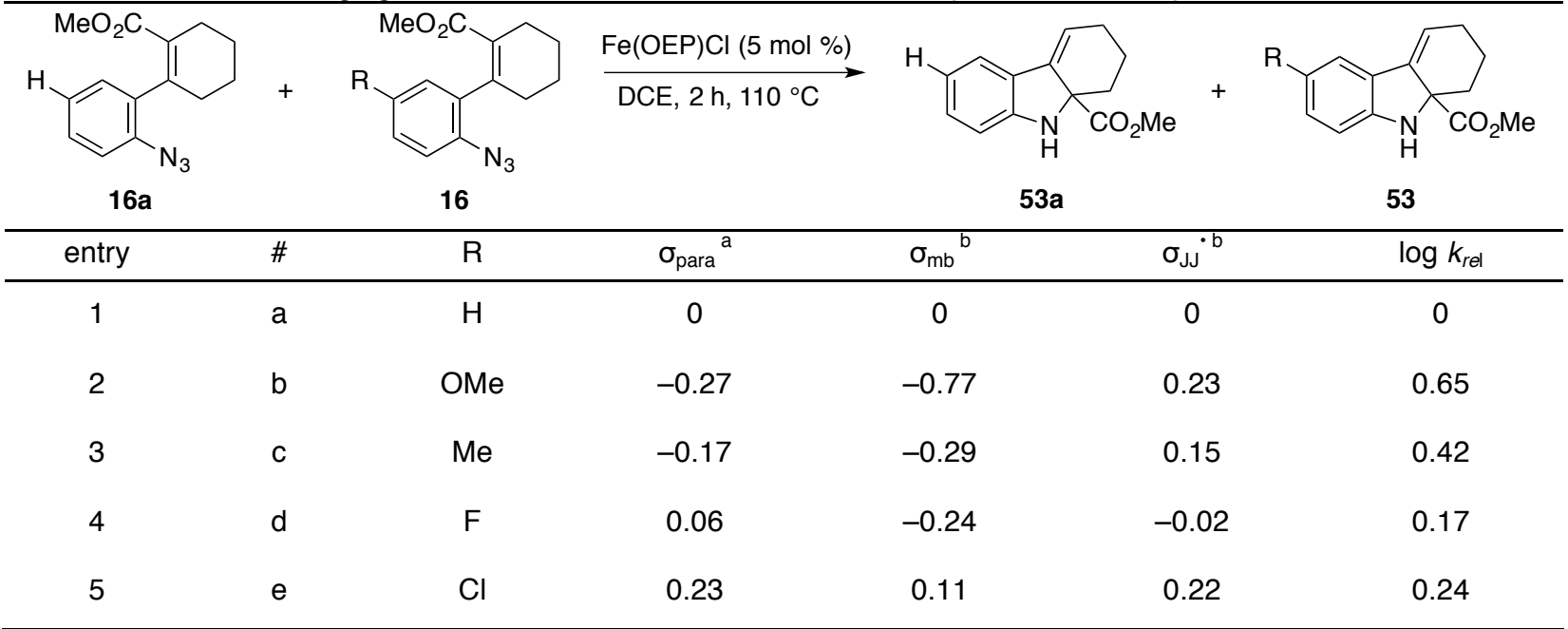

${ }^{a}$ From ref. 28. ${ }^{\text {b }}$ From ref. 29.

Figure s11. Correlation of relative rates between substituted styryl azides 16 and $16 a$ with $\sigma_{\text {para-constants. }}$

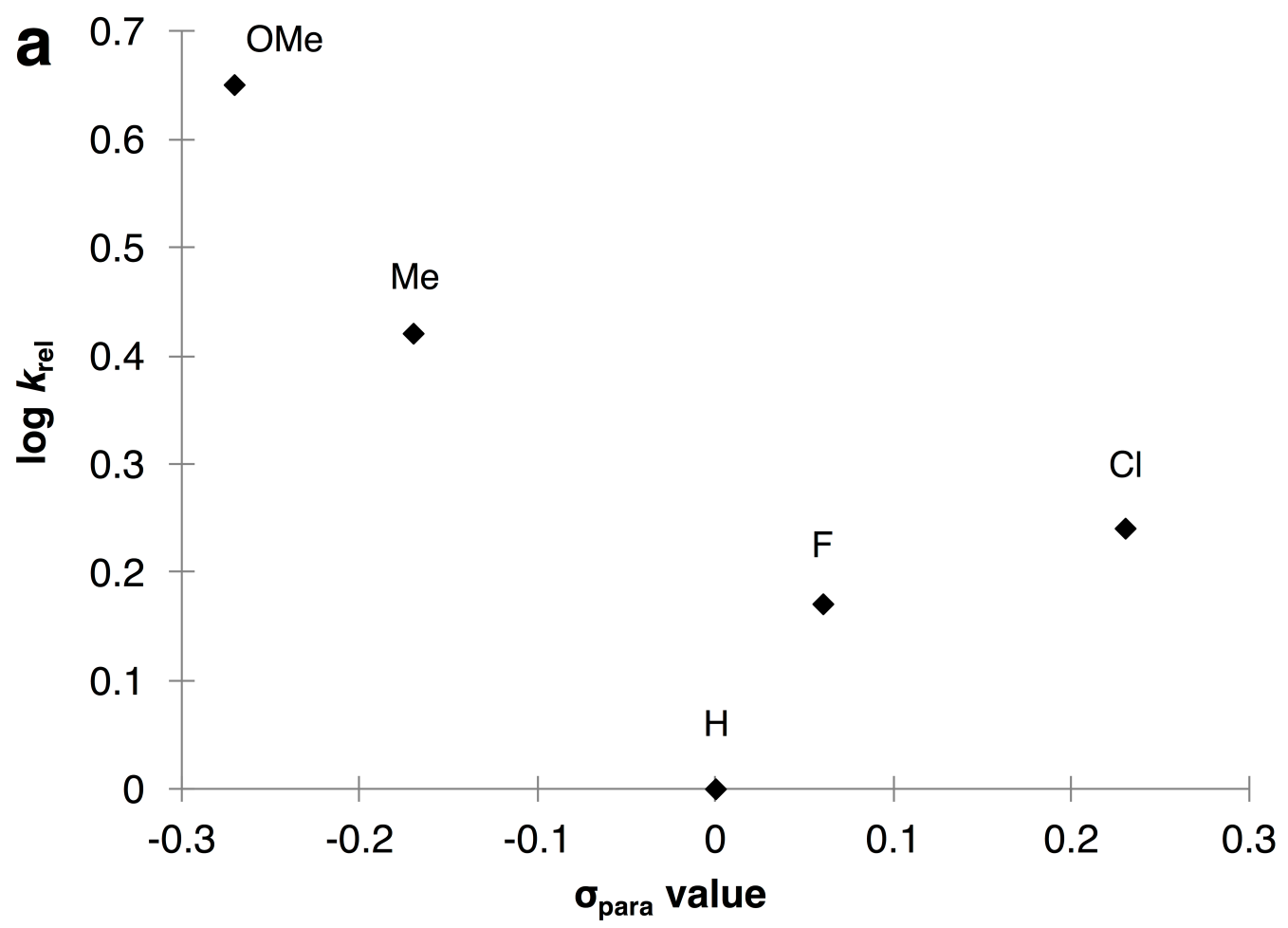


Figure s12. Correlation of relative rates between substituted styryl azides 16 and 16a with Jiang and Ji's $\sigma_{\mathrm{mb}}$ and $\sigma_{\mathrm{Jj}}$ values.

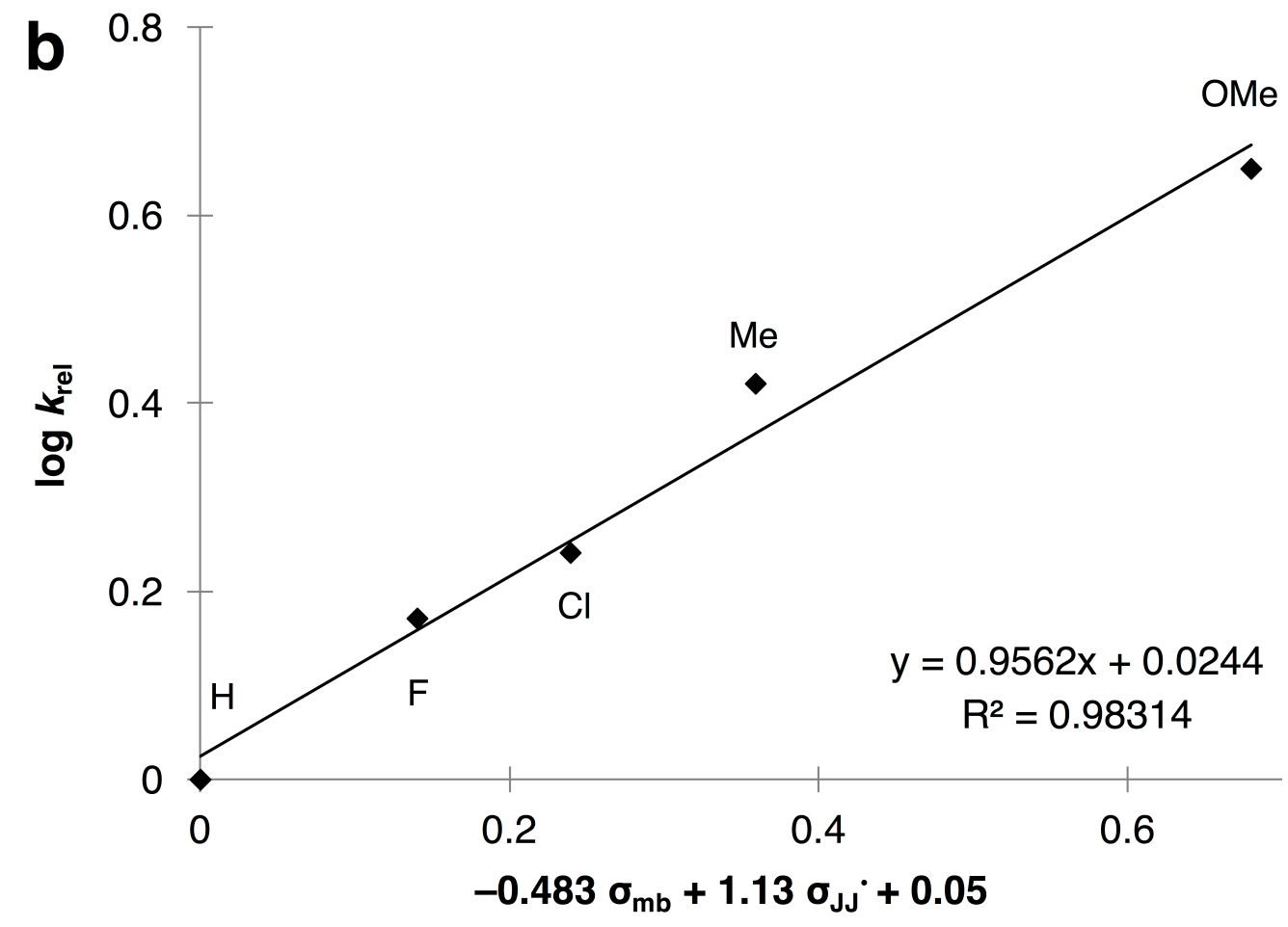

XI. $\quad \mathrm{Fe}(\mathrm{OEP}) \mathrm{Cl}-\mathrm{Catalyzed} \mathrm{Synthesis} \mathrm{of} 2 \mathrm{H}$-Indoles.

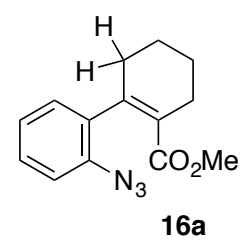

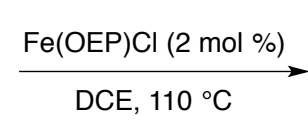

$16 a$

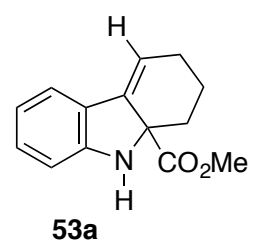

(s53)

Alkylidene indoline 53a. To a mixture $0.029 \mathrm{~g}$ of styryl azide $16 \mathbf{a}(0.11 \mathrm{mmol})$ and $\mathrm{Fe}(\mathrm{OEP}) \mathrm{Cl}(2 \mathrm{~mol} \%)$ was added 1.1 $\mathrm{mL}$ of toluene $(0.1 \mathrm{M})$. The resulting mixture was heated at $110{ }^{\circ} \mathrm{C}$. After $16 \mathrm{~h}$, the mixture was cooled to room temperature, diluted with $\mathrm{CH}_{2} \mathrm{Cl}_{2}$ and concentrated in vacuo. Purification of the residue by MPLC (3:97 - 30:70 EtOAc:hexanes) using silica gel afforded the product as a yellow solid $(0.018 \mathrm{~g}, 70 \%)$ : ${ }^{1} \mathrm{H} \mathrm{NMR}\left(500 \mathrm{MHz}, \mathrm{CDCl}_{3}\right) \delta$ $7.27(\mathrm{~d}, J=7.5 \mathrm{~Hz}, 1 \mathrm{H}), 7.05(\mathrm{t}, J=8.0 \mathrm{~Hz}, 1 \mathrm{H}), 6.76(\mathrm{t}, J=7.5 \mathrm{~Hz}, 1 \mathrm{H}), 6.68(\mathrm{~d}, J=8.0 \mathrm{~Hz}, 1 \mathrm{H}), 6.07(\mathrm{t}, J=4 \mathrm{~Hz}, 1 \mathrm{H})$, $4.80(\mathrm{~s}, 1 \mathrm{H}), 3.69(\mathrm{~s}, 3 \mathrm{H}), 2.37-2.29(\mathrm{~m}, 3 \mathrm{H}), 1.82-1.76(\mathrm{~m}, 3 \mathrm{H}) ;{ }^{13} \mathrm{C}$ NMR $\left(125 \mathrm{MHz}, \mathrm{CDCl}_{3}\right) \delta 175.4(\mathrm{C}), 150.2(\mathrm{C})$, $137.0(\mathrm{C}), 128.8(\mathrm{CH}), 127.0(\mathrm{C}), 120.3(\mathrm{CH}), 120.0(\mathrm{CH}), 119.8(\mathrm{CH}), 110.7(\mathrm{CH}), 69.2(\mathrm{C}), 52.7\left(\mathrm{CH}_{3}\right), 32.2\left(\mathrm{CH}_{2}\right)$, $24.0\left(\mathrm{CH}_{2}\right), 18.1\left(\mathrm{CH}_{2}\right)$; ATR-FTIR (thin film): 3341, 2936, 2904, 1713, 1603, 1462, 1449, 1255, $1232 \mathrm{~cm}^{-1}$. HRMS (ES) $m / z$ calculated for $\mathrm{C}_{14} \mathrm{H}_{16} \mathrm{NO}_{2}[\mathrm{M}+\mathrm{H}]^{+}: 230.1181$, found: 230.1188 . 

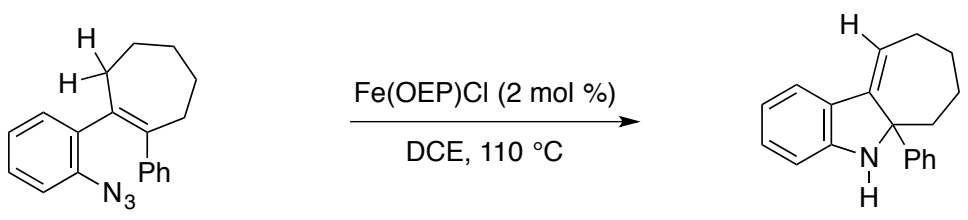

(s54)

55

56

Alkylidene indoline 56. To a mixture of $0.026 \mathrm{~g}$ of styryl azide $55(0.088 \mathrm{mmol})$ and $\mathrm{Fe}(\mathrm{OEP}) \mathrm{Cl}(2 \mathrm{~mol} \%)$ was added $0.9 \mathrm{~mL}$ of toluene $(0.1 \mathrm{M})$. The resulting mixture was heated at $110{ }^{\circ} \mathrm{C}$. After $16 \mathrm{~h}$, the mixture was cooled to room temperature, diluted with $\mathrm{CH}_{2} \mathrm{Cl}_{2}$ and concentrated in vacuo. Purification of the residue by MPLC (3:97 - 30:70 EtOAc:hexanes) using silica gel afforded the product as a yellow oil $(0.018 \mathrm{~g}, 77 \%)$ : ${ }^{1} \mathrm{H}$ NMR $\left(500 \mathrm{MHz}, \mathrm{CDCl}_{3}\right) \delta 7.46$ $(\mathrm{dd}, J=8.5 \mathrm{~Hz}, 1.5 \mathrm{~Hz}, 2 \mathrm{H}), 7.37-7.33(\mathrm{~m}, 3 \mathrm{H}), 7.27(\mathrm{~m}, 1 \mathrm{H}), 7.00(\mathrm{dt}, J=8.0 \mathrm{~Hz}, 1.0 \mathrm{~Hz}, 1 \mathrm{H}), 6.72(\mathrm{dt}, J=7.5 \mathrm{~Hz}, 1.0$ $\mathrm{Hz}, 1 \mathrm{H}), 6.56(\mathrm{~m}, 1 \mathrm{H}), 6.48(\mathrm{~d}, J=8.0 \mathrm{~Hz}, 1 \mathrm{H}), 3.96(\mathrm{~s}, 1 \mathrm{H}), 2.58-2.54(\mathrm{~m}, 1 \mathrm{H}), 2.20(\mathrm{~m}, 1 \mathrm{H}), 2.13-2.07(\mathrm{~m}, 2 \mathrm{H}), 1.88$ $-1.85(\mathrm{~m}, 1 \mathrm{H}), 1.65(\mathrm{~m}, 1 \mathrm{H}), 1.49-1.44(\mathrm{~m}, 2 \mathrm{H}) ;{ }^{13} \mathrm{C}$ NMR $\left(125 \mathrm{MHz}, \mathrm{CDCl}_{3}\right) \delta 150.1(\mathrm{C}), 146.2(\mathrm{C}), 142.6(\mathrm{C}), 129.0$ $(\mathrm{CH}), 128.9(\mathrm{CH}), 128.6(\mathrm{C}), 127.0(\mathrm{CH}), 126.1(\mathrm{CH}), 120.4(\mathrm{CH}), 119.6(\mathrm{CH}), 118.9(\mathrm{CH}), 110.6(\mathrm{CH}), 72.6(\mathrm{C}), 38.8$ $\left(\mathrm{CH}_{2}\right), 28.2\left(\mathrm{CH}_{2}\right), 27.9\left(\mathrm{CH}_{2}\right), 27.2\left(\mathrm{CH}_{2}\right)$; ATR-FTIR (thin film): 3342, 3056, 2928, 2859, 1703, 1600, 1445, 1391, 1264 $\mathrm{cm}^{-1}$. HRMS (ES) $\mathrm{m} / z$ calculated for $\mathrm{C}_{19} \mathrm{H}_{20} \mathrm{~N}[\mathrm{M}+\mathrm{H}]^{+}: 262.1596$, found: 262.1599 .

\section{References}

1. Pangborn, A. B.; Giardello, M. A.; Grubbs, R. H.; Rosen, R. K.; Timmers, F. J. Organometallics 1996, $15,1518$. 2. Sun, K.; Sachwani, R.; Richert, K. J.; Driver, T. G. Org. Lett. 2009, 11, 3598.

3. Zhang, F.; Moses, J. E. Org. Lett. 2009, 11, 1587.

4. (a) Sun, K.; Liu, S.; Bec, P. M.; Driver, T. G. Angew. Chem. Int. Ed. 2011, 50, 1702. (b) Nguyen, Q.; Sun, K.; Driver, T. G. J. Am. Chem. Soc. 2012, 134, 7262.

5. Kong, C.; Jana, N.; Driver, T. G. Org. Lett. 2013, 15, 824.

6. Preshlock, S. M.; Plattner, D. L.; Maligres, P. E.; Krska, S. W.; Maleczka, R. E.; Smith, M. R. Angew. Chem. Int. Ed. 2013, 52, 12915.

7. Jana, N.; Nguyen, Q.; Driver, T. G. J. Org. Chem. 2014, 79, 2781.

8. (a) Larock, R. C.; Reddy, C. K. Org. Lett. 2000, 2, 3325. (b) Larock, R. C.; Reddy, C. K. J. Org. Chem. $2002,67,2027$. 9. Jones, C.; Nguyen, Q.; Driver, T. G. Angew. Chem. Int. Ed. 2014, 53, 785.

10. Luzzio, F. A.; Fitch, R. W.; Moore, W. J.; Mudd, K. J. J. Chem. Educ. 1999, 76, 974.

11. Payette, J. N.; Yamamoto, H. J. Am. Chem. Soc. 2008, 130, 12276.

12. Chai, Y.; Wan, Z.-L.; Wang, B.; Guo, H.-Y.; Liu, M.-L. Eur.J. Med.Chem. 2009, 44, 4063.

13. Meyer, C.; Piva, O.; Pete, J.-P. Tetrahedron 2000, 56, 4479.

14. Moreau, R. J.; Sorensen, E. J. Tetrahedron 2007, 63, 6446.

15. Maeda, H.; Nishitsuji, N.; Mizuno, K. Res. Chem. Intermed. 2010, 36, 577.

16. Akimoto, R.; Tokugawa, T.; Yamamoto, Y.; Yamataka, H. J. Org. Chem. 2012, 77, 4073.

17. Strotman, N. A.; Sommer, S.; Fu, G. C. Angew. Chem. Int. Ed. 2007, 46, 3556.

18. Wheeler, T. N. J. Org. Chem. 1979, 44, 4906.

19. Ishikawa, T.; Kadoya, R.; Arai, M.; Takahashi, H.; Kaisi, Y.; Mizuta, T.; Yoshikai, K.; Saito, S. J. Org. Chem. 2001, 66,8000 .

20. Scheiper, B.; Bonnekessel, M.; Krause, H.; Fürstner, A. J. Org. Chem. 2004, 69, 3943.

21. Rodríguez, I.; Calaza, M. I.; Jiménez, A. I.; Cativiela, C. Tetrahedron 2012, 68, 9578.

22. Kunzmann, M. H.; Bach, N. C.; Bauer, B.; Sieber, S. A. Chem. Sci. 2014, 5, 1158.

23. Lim, B.-Y.; Jung, B.-E.; Cho, C.-G. Org. Lett. 2014, 16, 4492.

24. Petersen, M. D.; Boye, S. V.; Nielsen, E. H.; Willumsen, J.; Sinning, S.; Wiborg, O.; Bols, M. Bioorg. Med. Chem. 2007, 15, 4159 . 
25. Micheli, F.; Cavanni, P.; Andreotti, D.; Arban, R.; Benedetti, R.; Bertani, B.; Bettati, M.; Bettelini, L.; Bonanomi, G.; Braggio, S.; Carletti, R.; Checchia, A.; Corsi, M.; Fazzolari, E.; Fontana, S.; Marchioro, C.; Merlo-Pich, E.; Negri, M.; Oliosi, B.; Ratti, E.; Read, K. D.; Roscic, M.; Sartori, I.; Spada, S.; Tedesco, G.; Tarsi, L.; Terreni, S.; Visentini, F.; Zocchi, A.; Zonzini, L.; Di Fabio, R. J. Med. Chem. 2010, 53, 4989.

26. Kong, C.; Driver, T. G. Org. Lett. 2015, 17, 802.

27. (a) Barral, K.; Moorhouse, A. D.; Moses, J. E. Org. Lett. 2007, 9, 1809. (b) Zhang, F.; Moses, J. E. Org. Lett. 2009, 11 , 1587.

28. Hansch, C.; Leo, A.; Taft, R. W. Chem. Rev. 1991, 91, 165.

29. Jiang, X.; Ji, G. J. Org. Chem. 1992, 57, 6051.

30. Dust, J. M.; Arnold, D. R. J. Am. Chem. Soc. 1983, 105, 1221.

31. Creary, X.; Mehrsheikh-Mohammadi, M. E.; McDonald, S. J. Org. Chem. 1987, 52, 3254.

32. Dincturk, S.; Jackson, R. A. J. Chem. Soc., Perkin Trans. 2 1981, 1127. 


\section{Spectral Data}

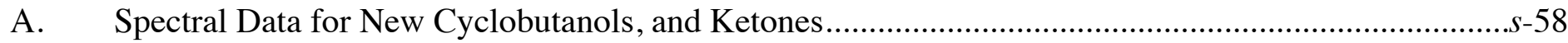

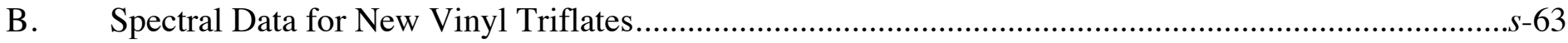

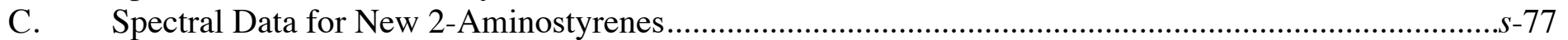

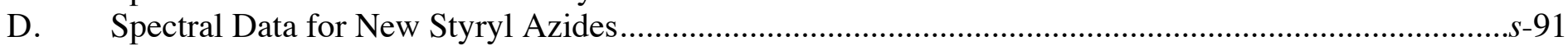

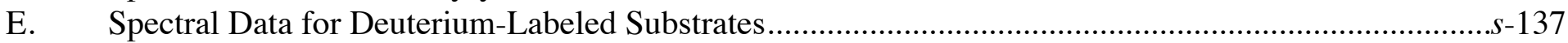

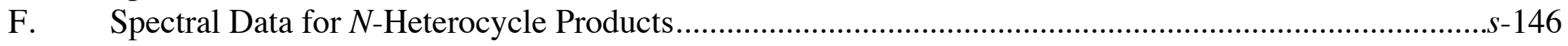

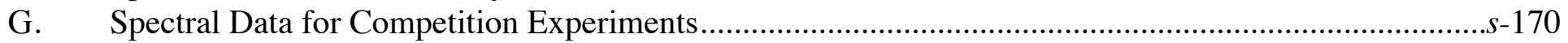




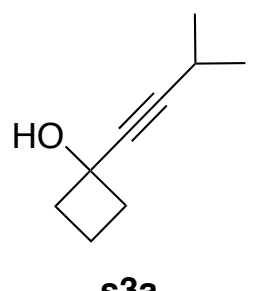

s3a

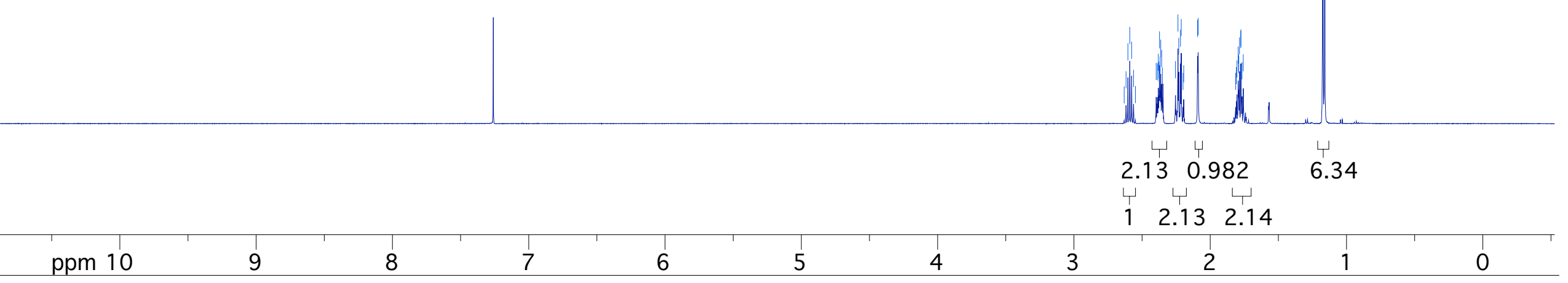




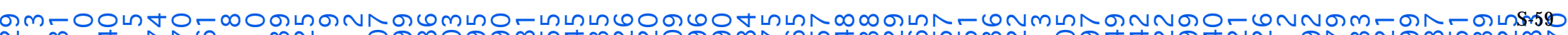
드-

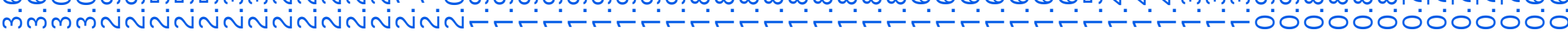
$L L L L L L L$<smiles>COC(=O)C1C(=O)[C@H]([InH])CC[C@H]1C</smiles>

s4a 


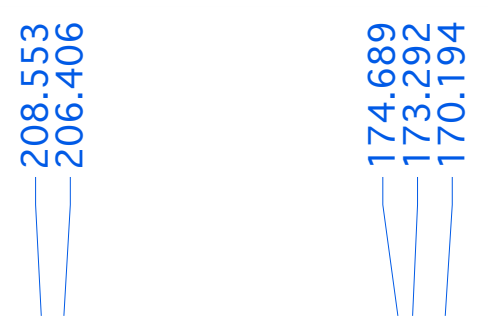

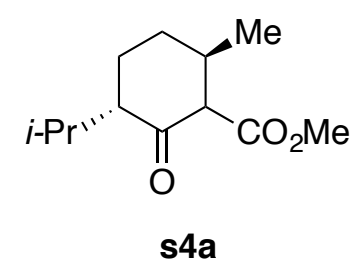

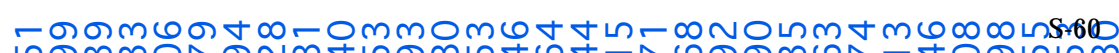

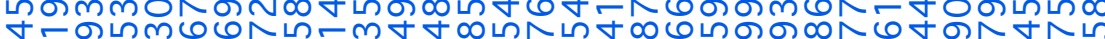
AN 


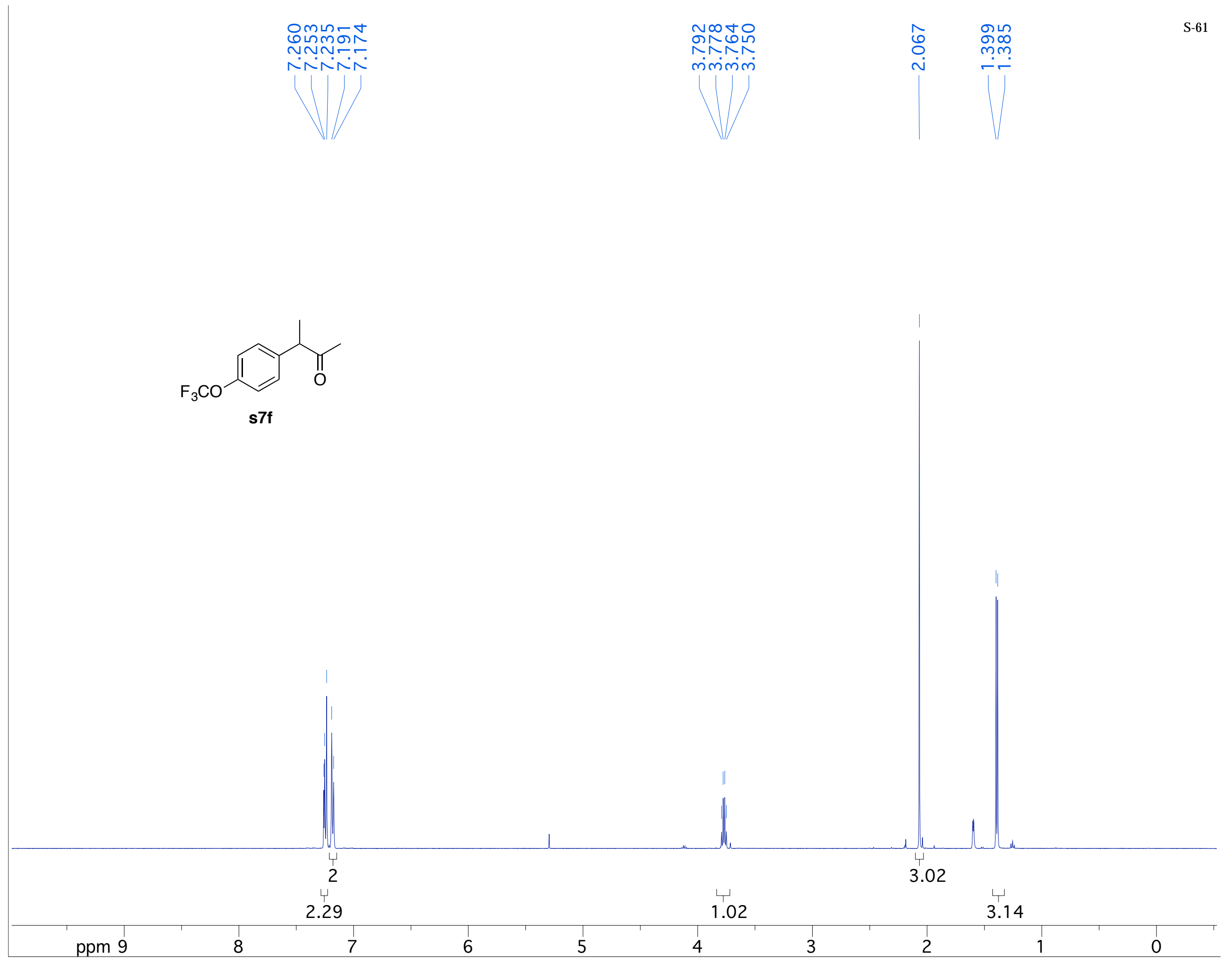



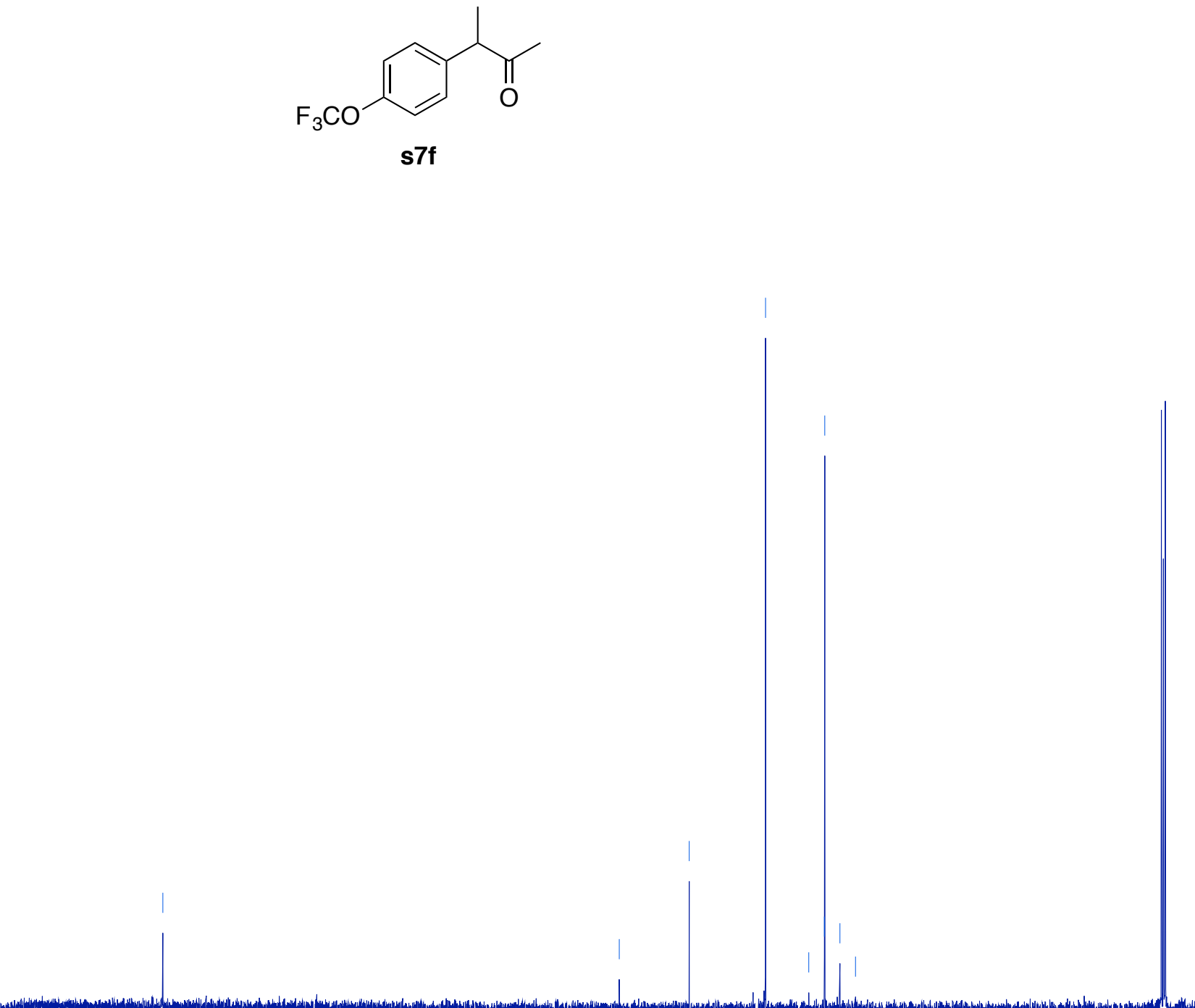


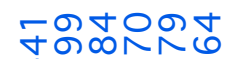

รmmm-?

NNNNN

$+1)$

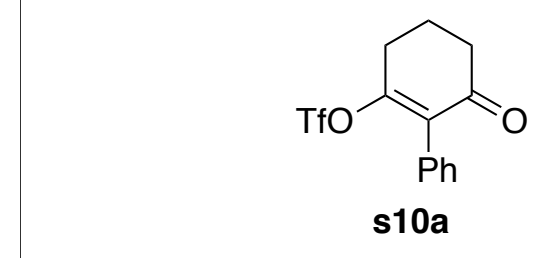

แกำกㄴ. ด๐ை

ن

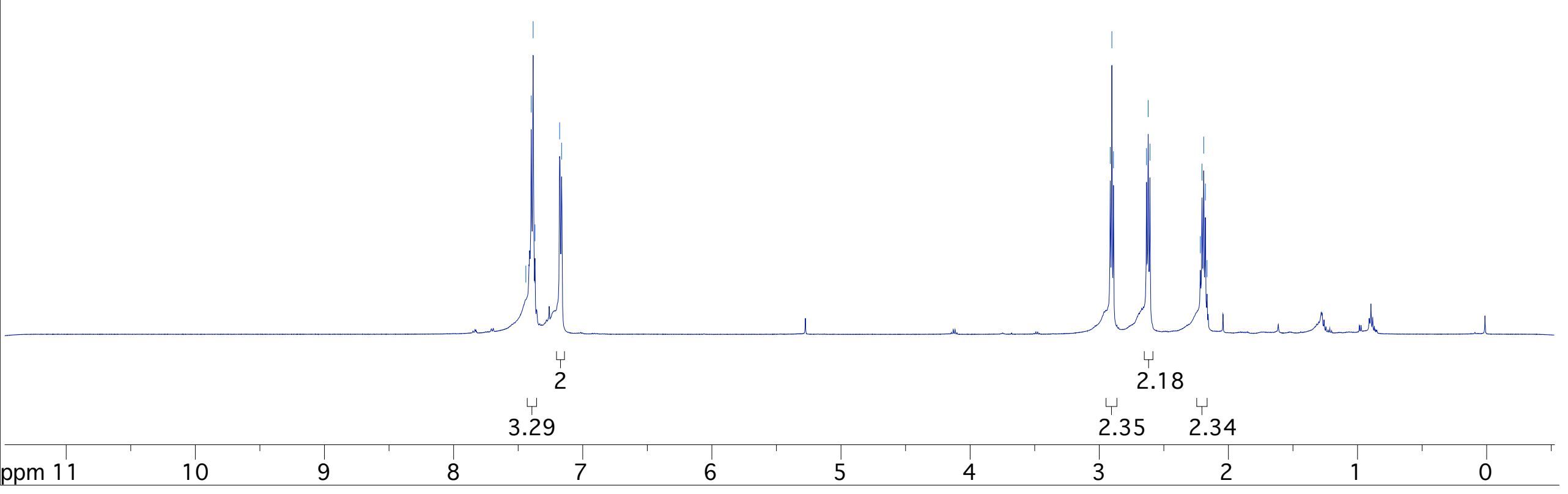




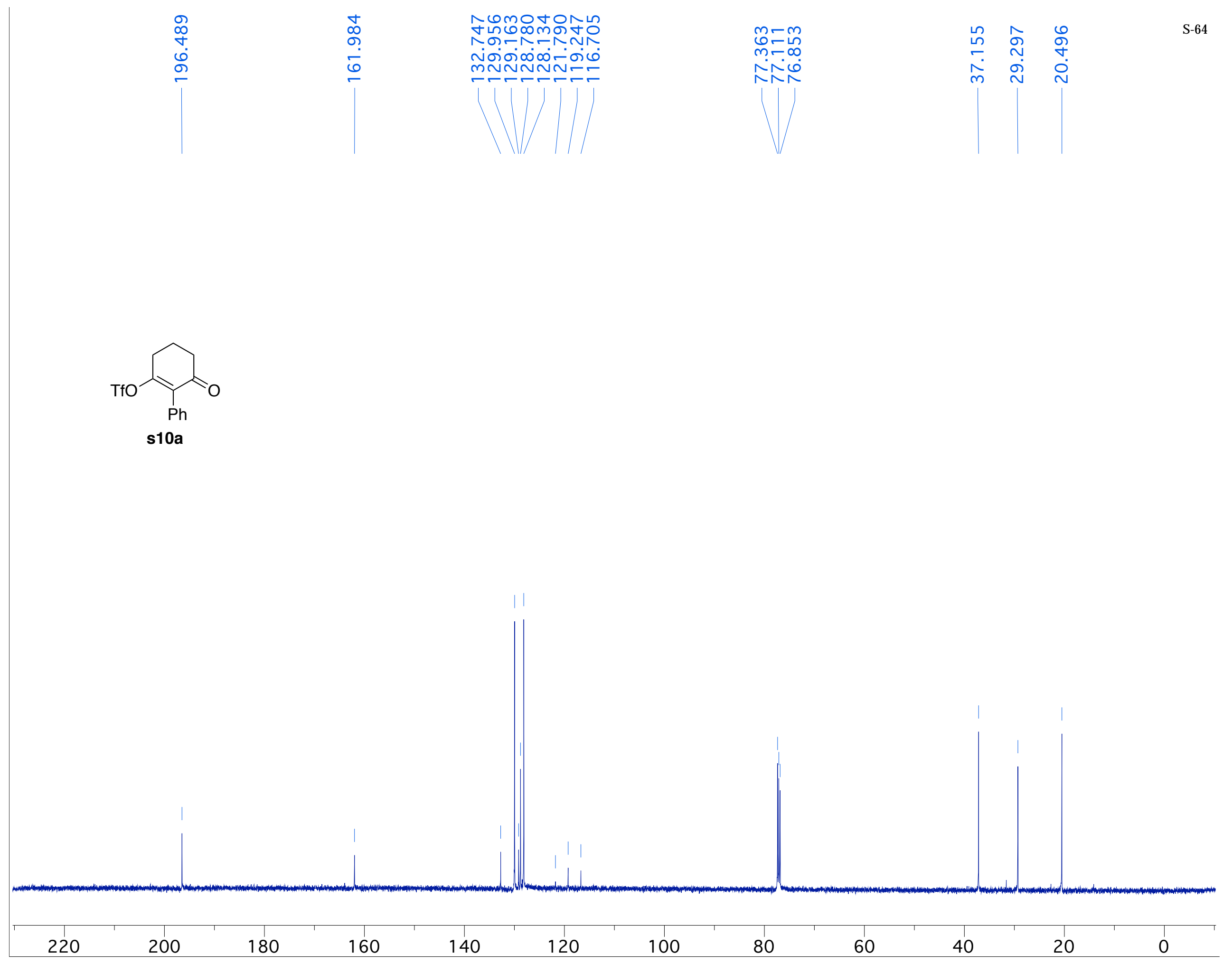




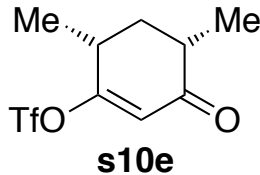

$$
\text { s10e }
$$

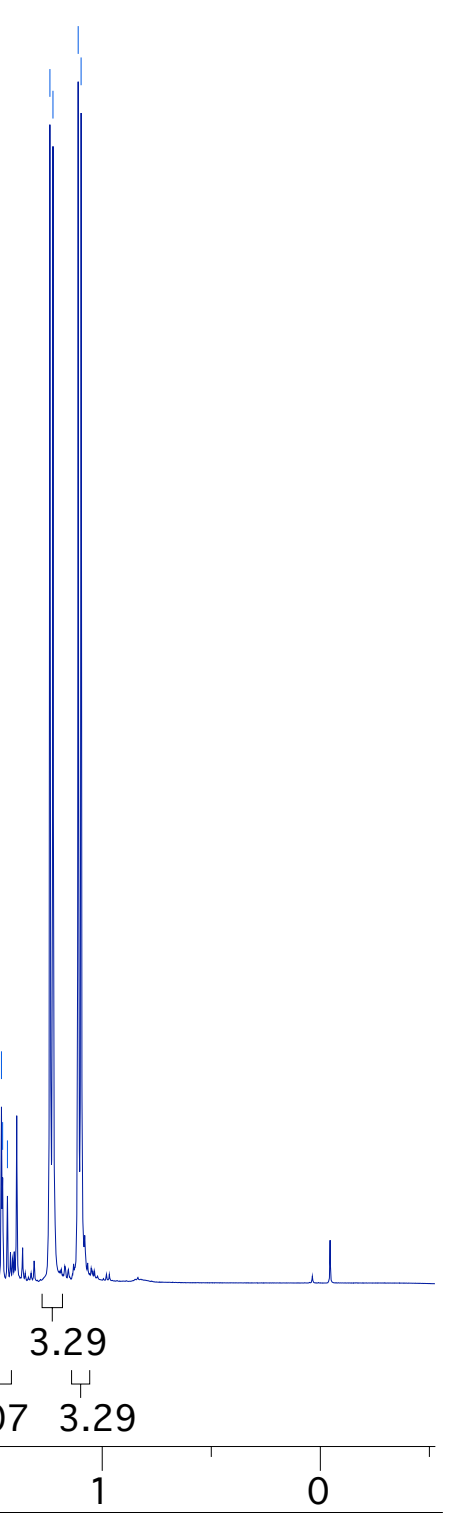




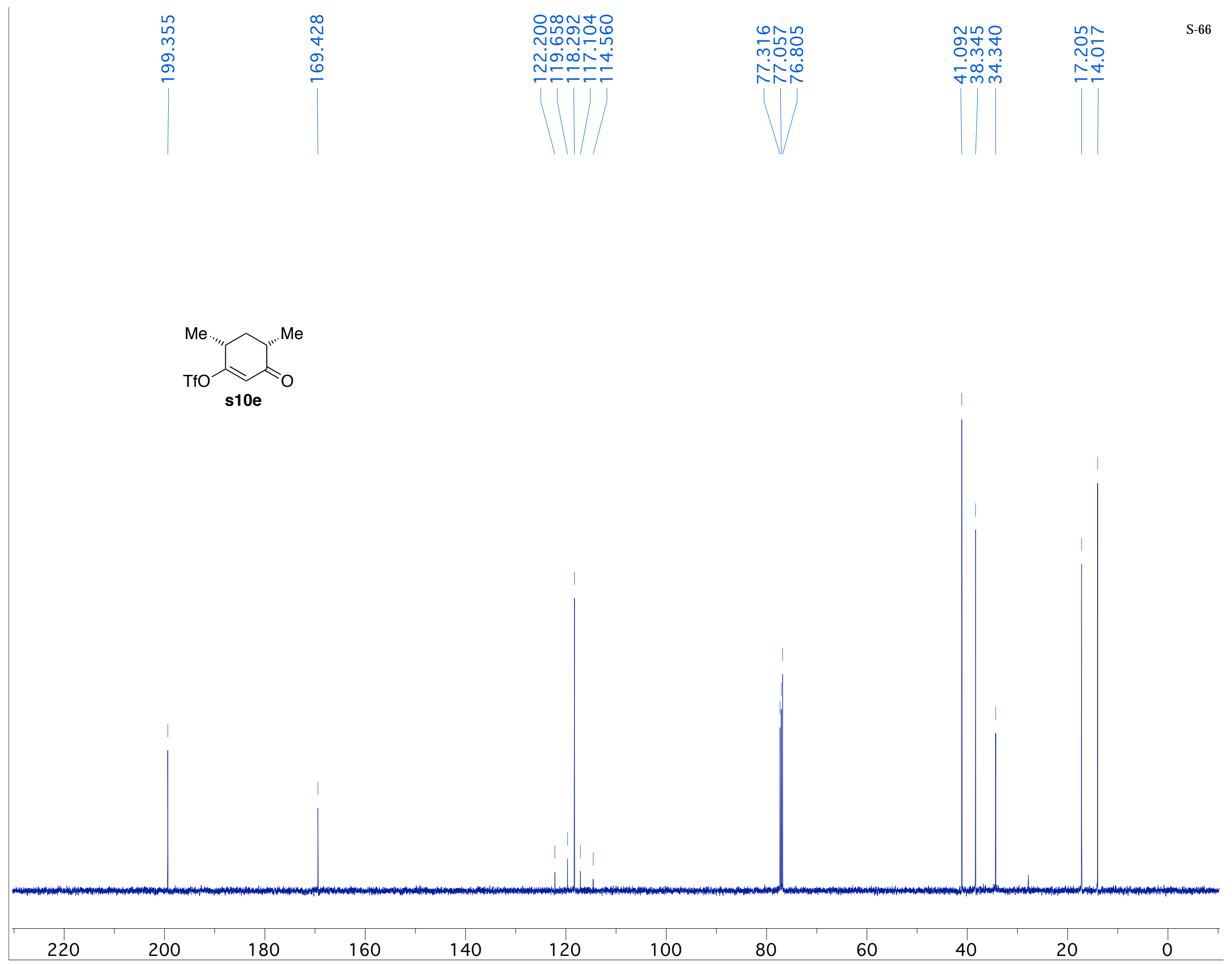




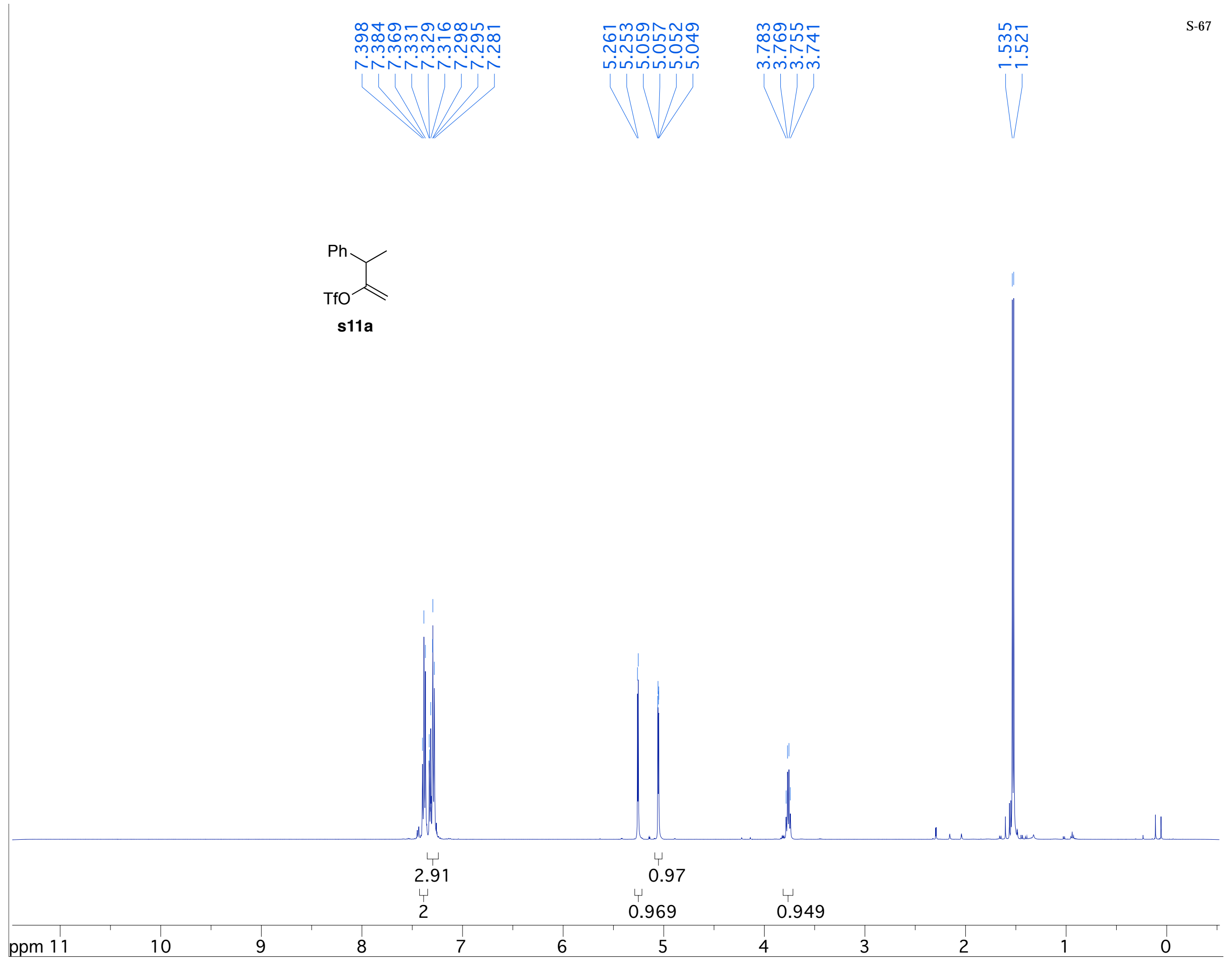




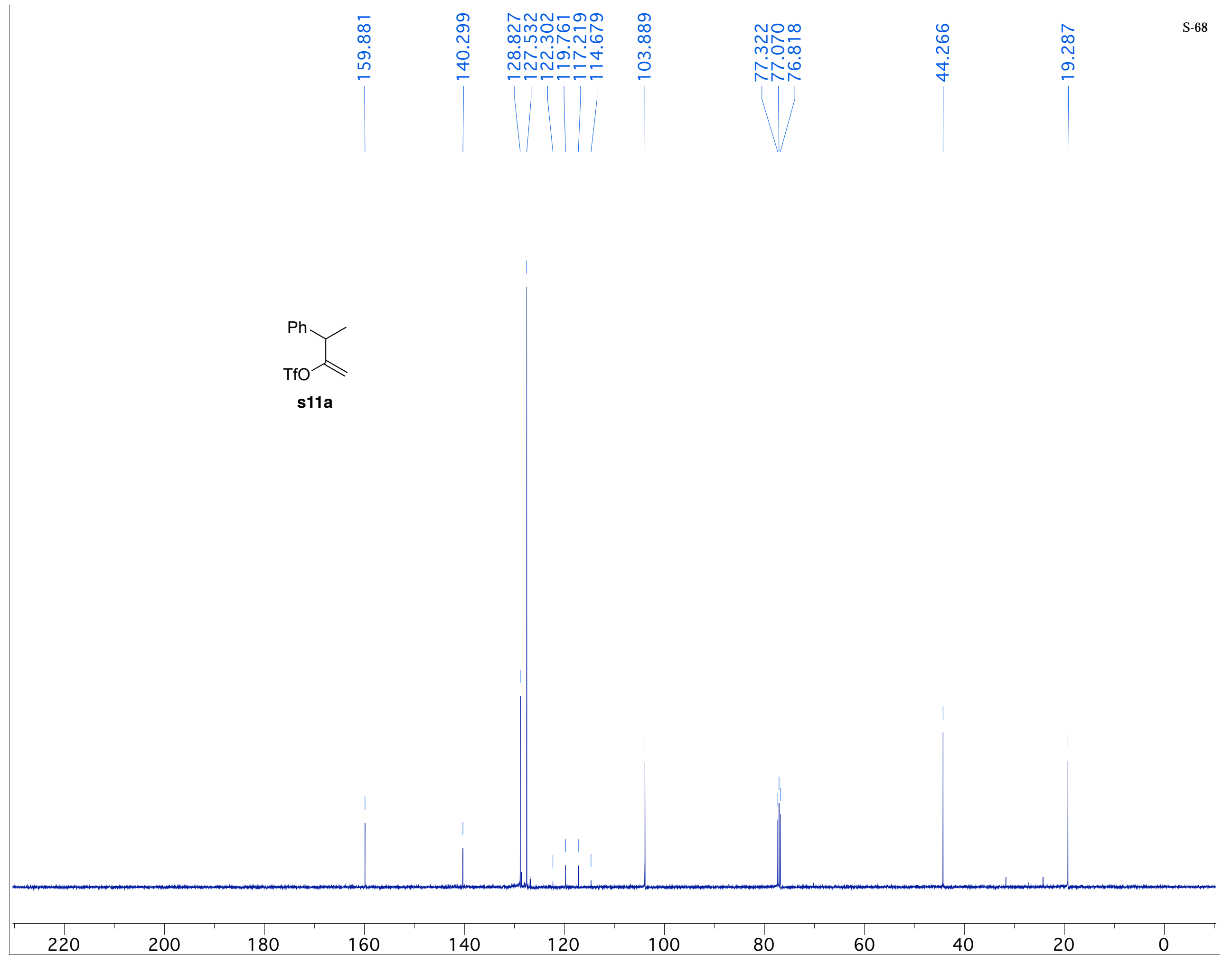




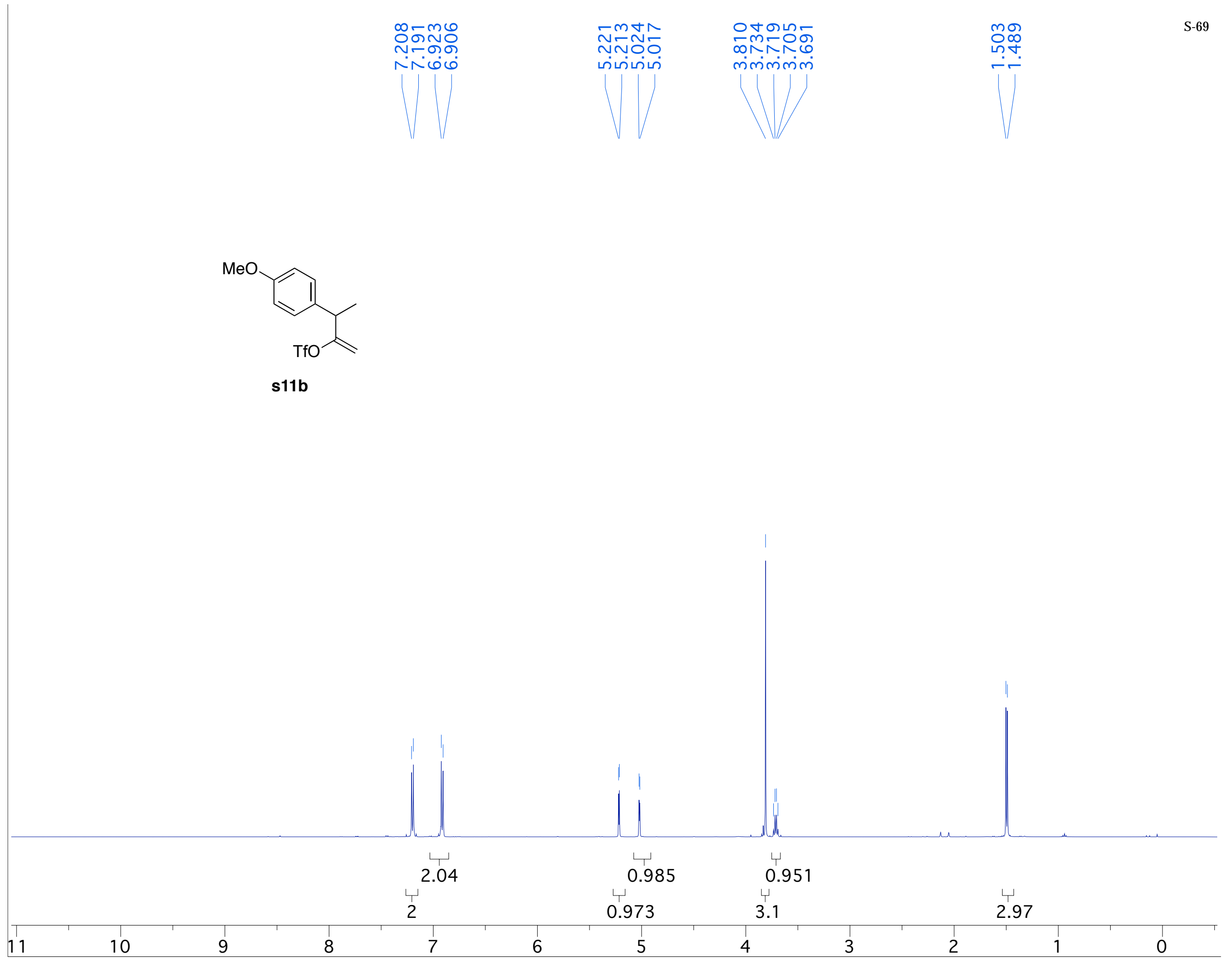




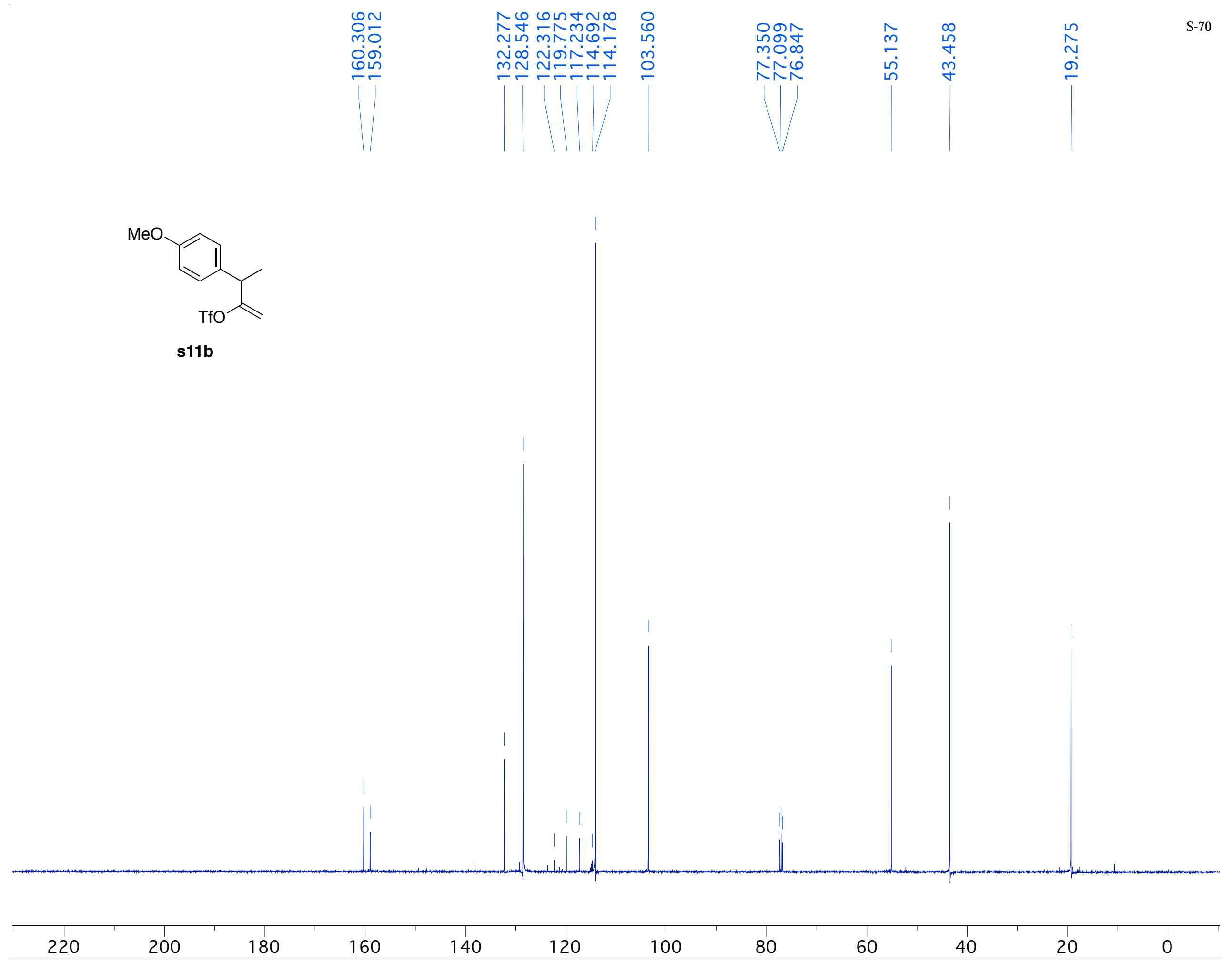



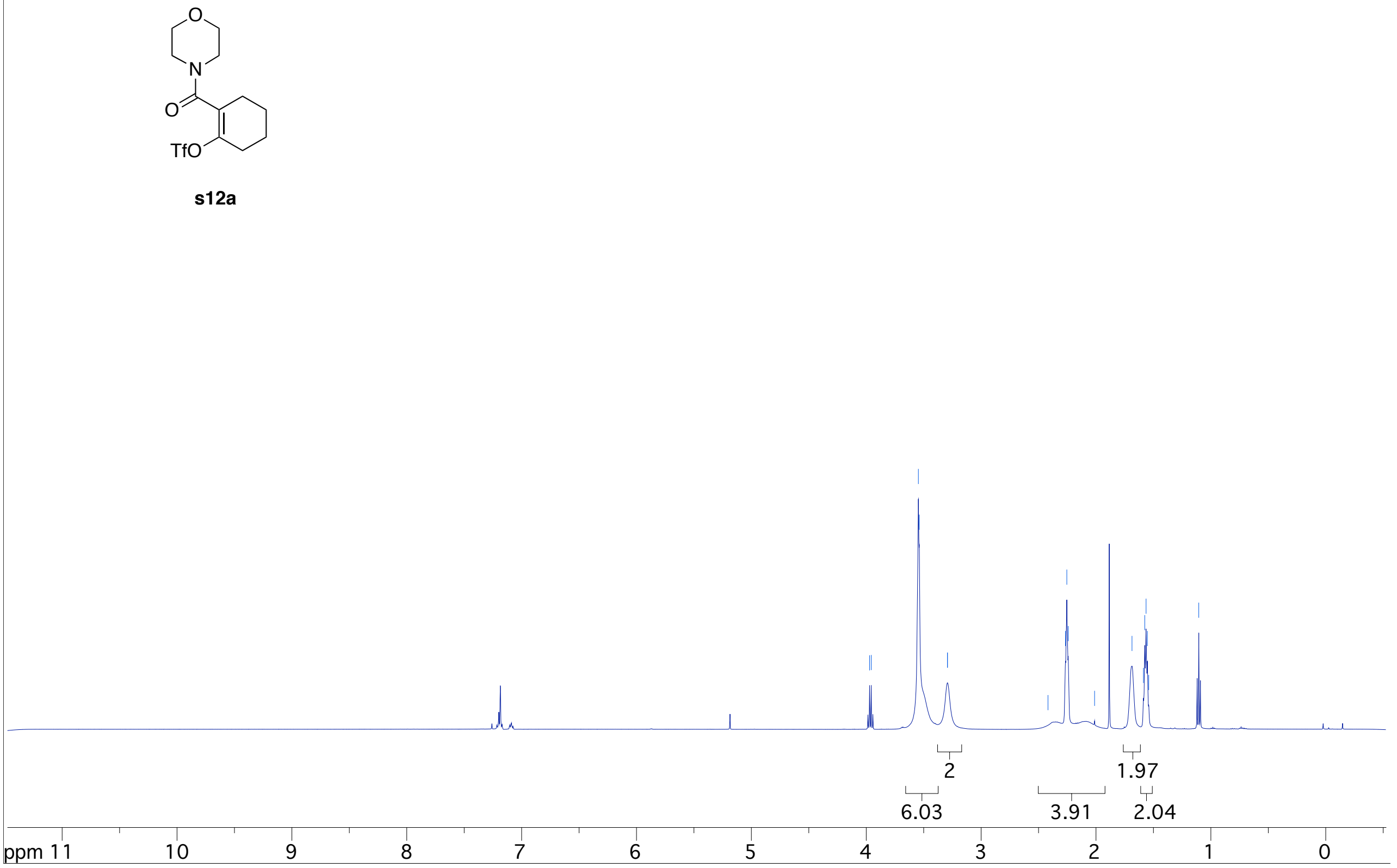


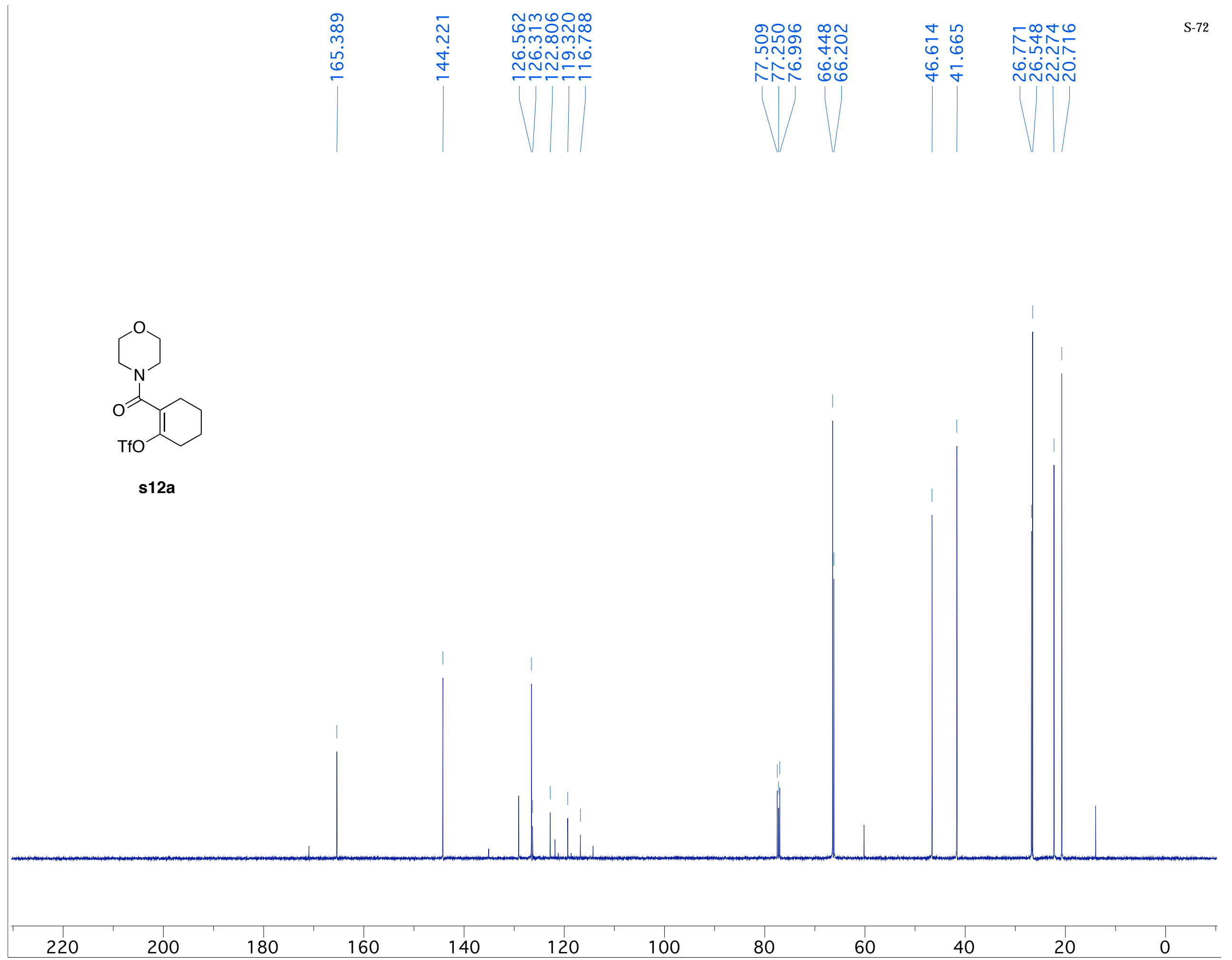



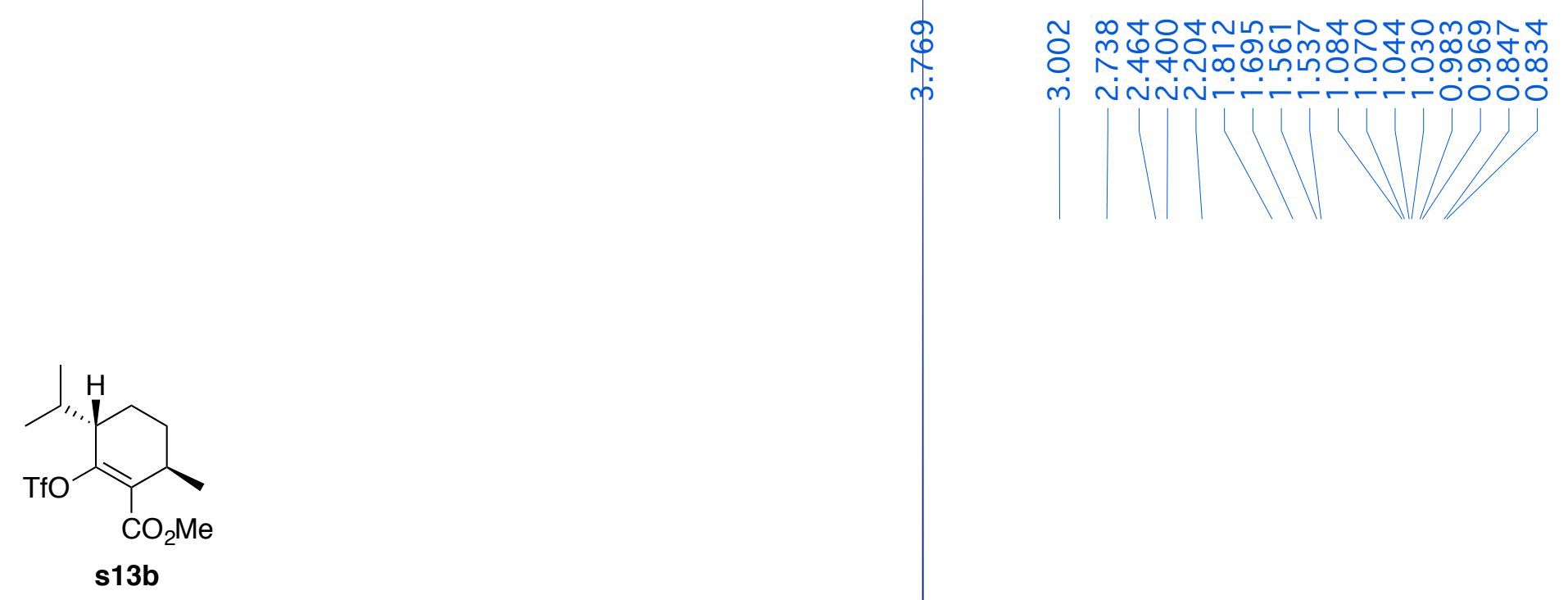

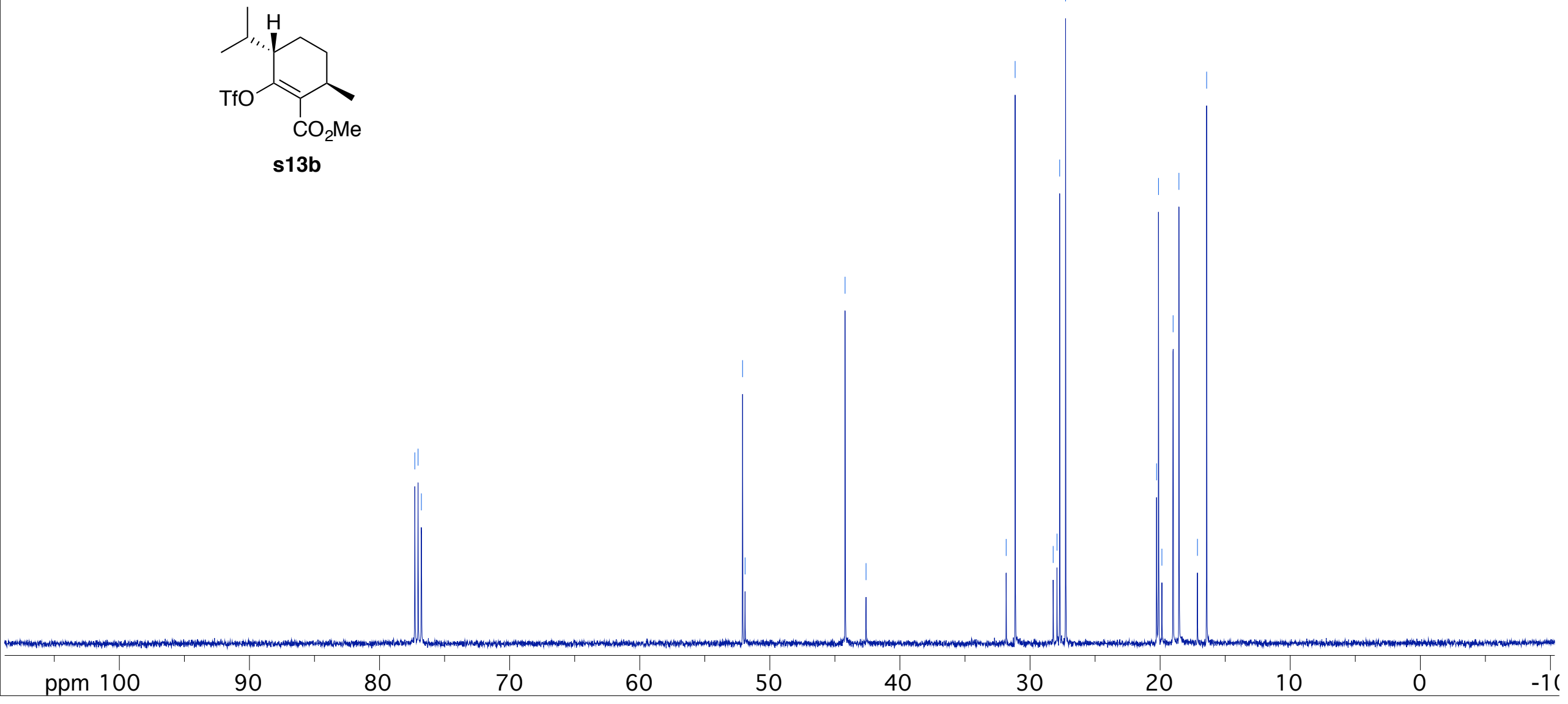

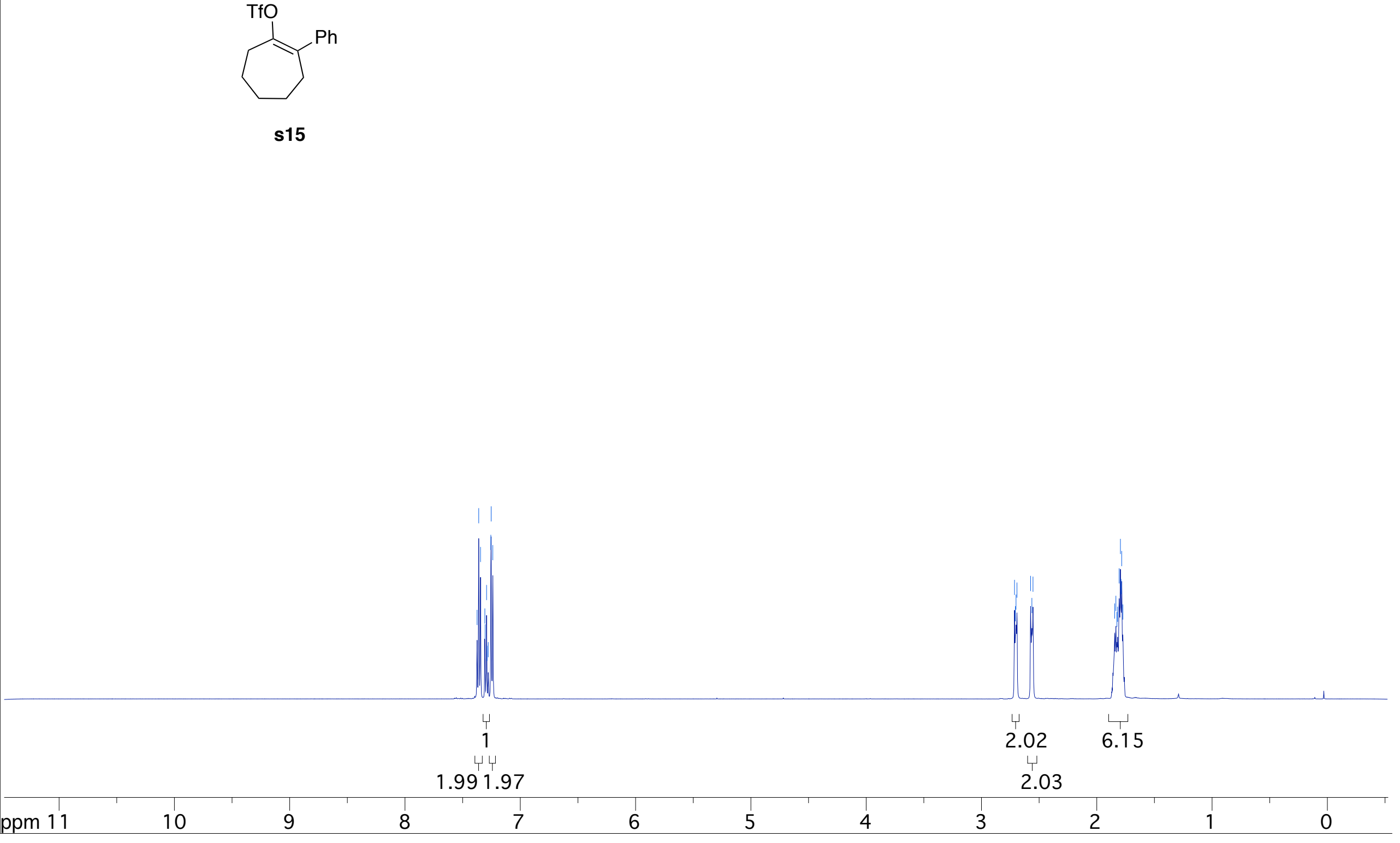


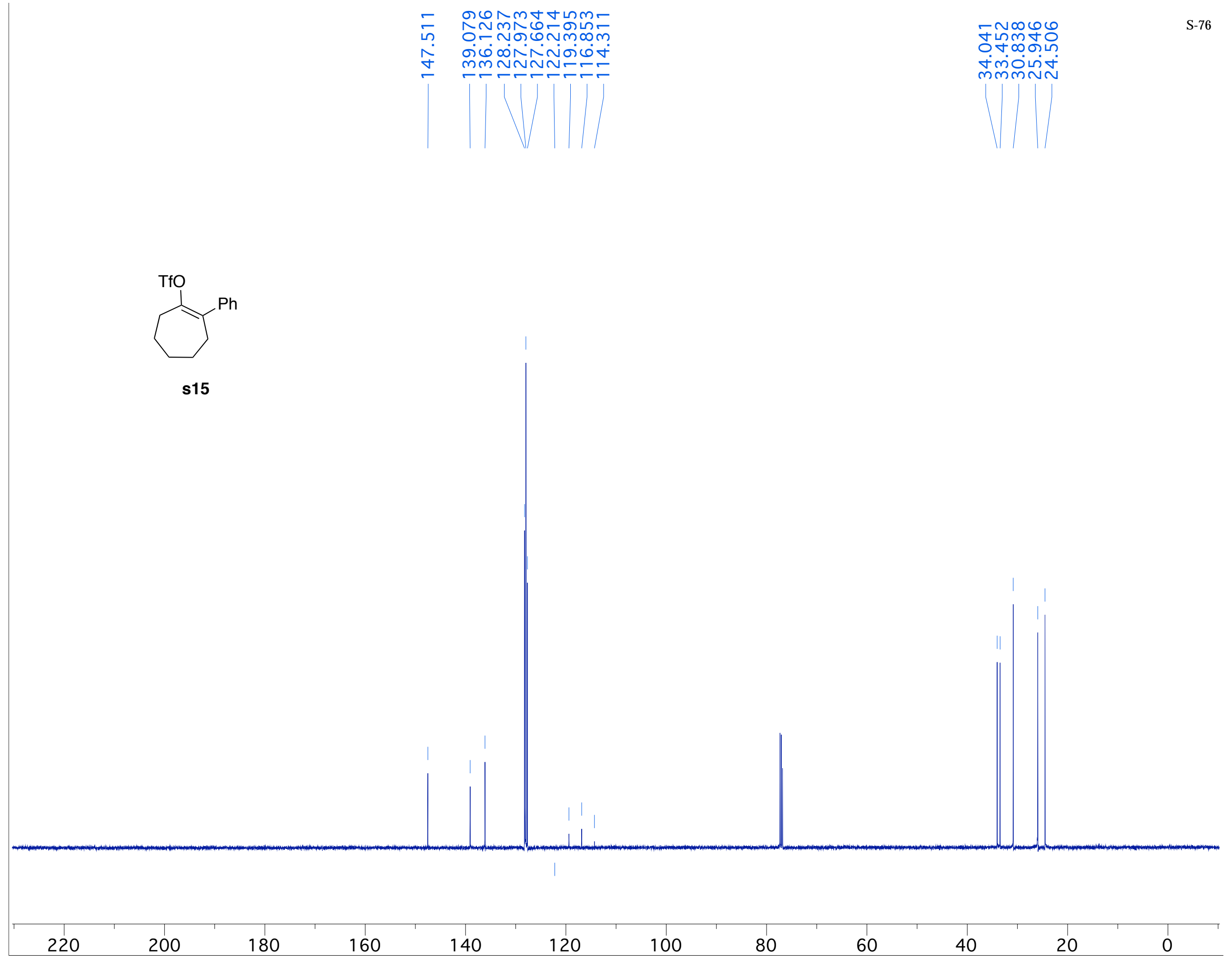




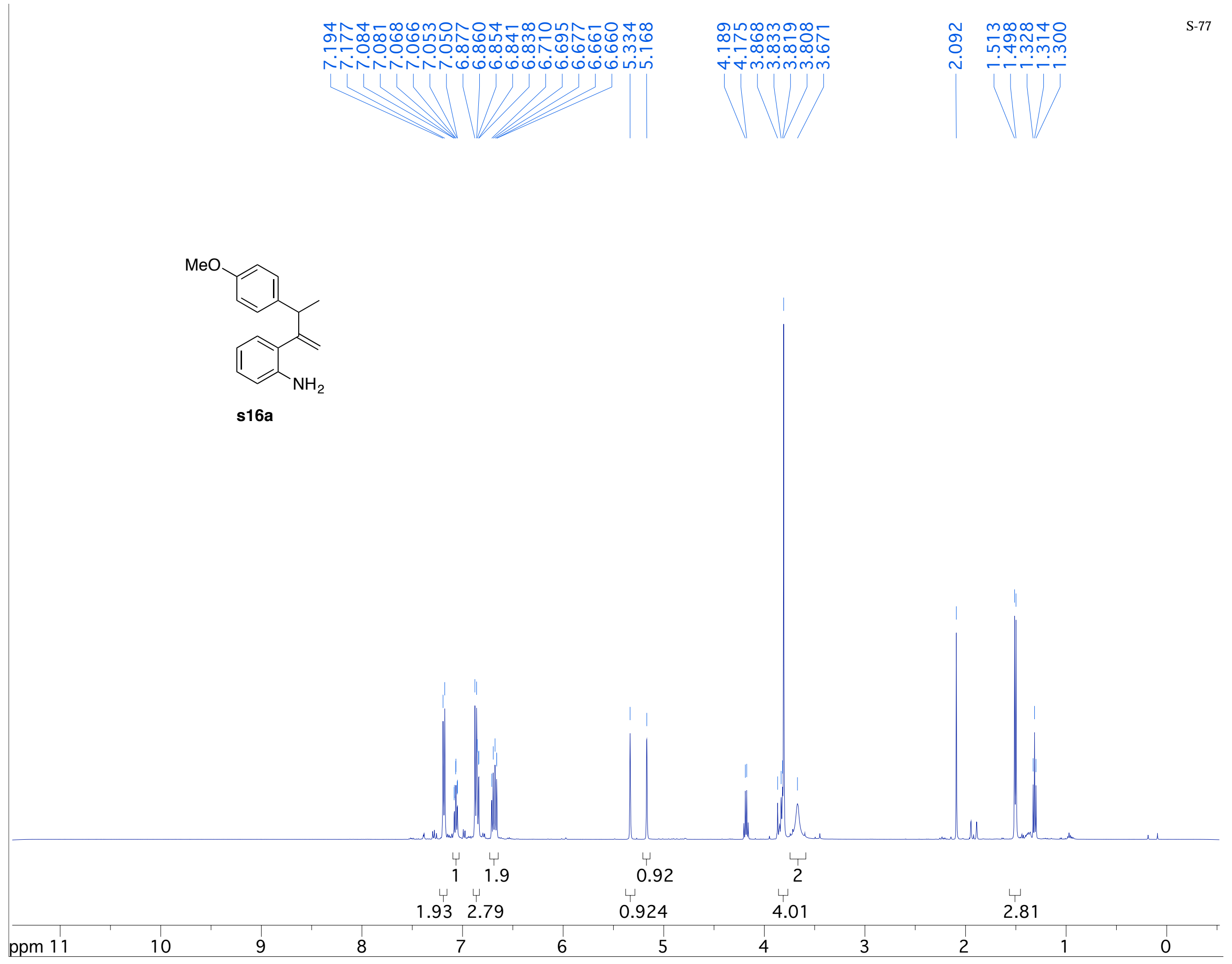




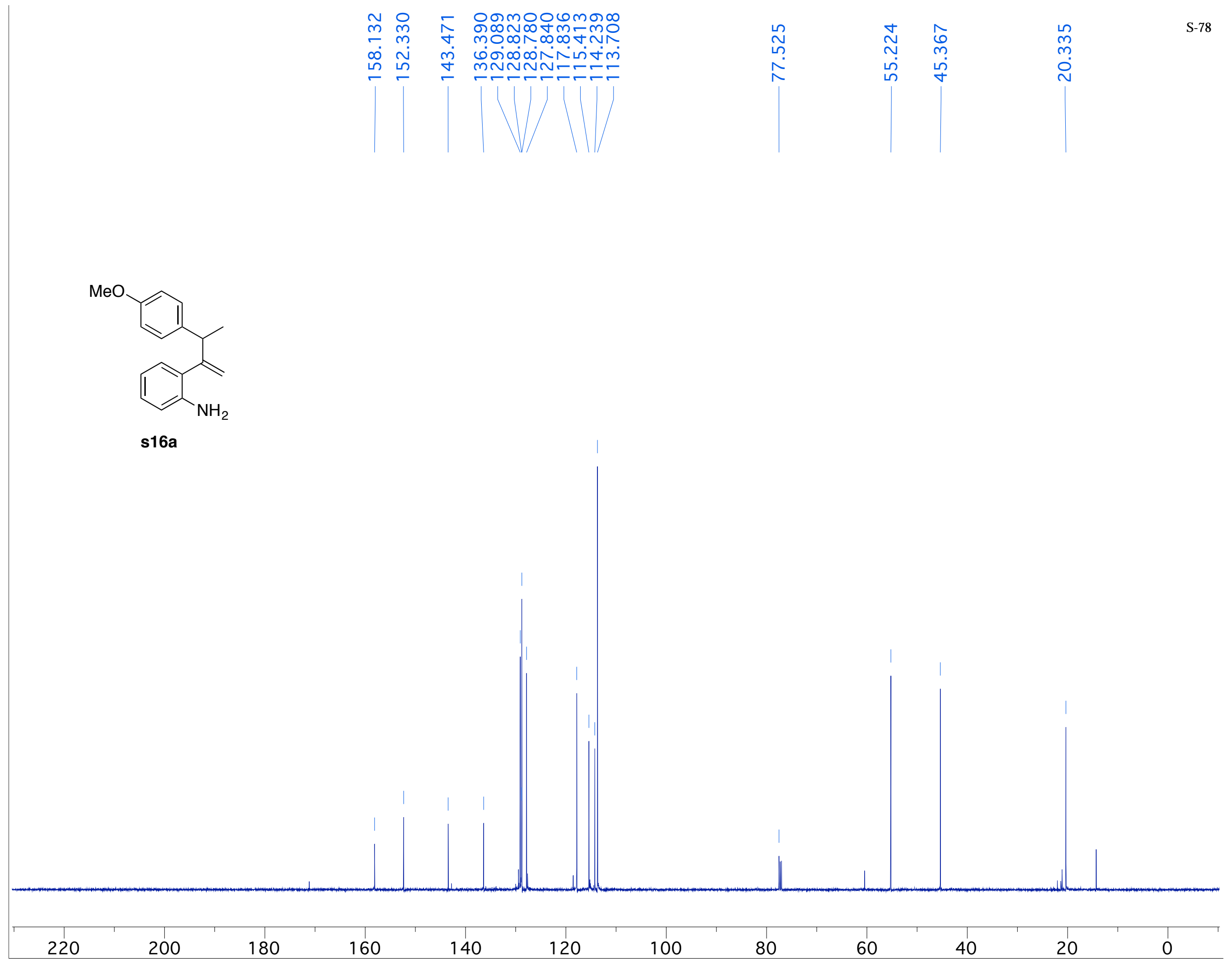




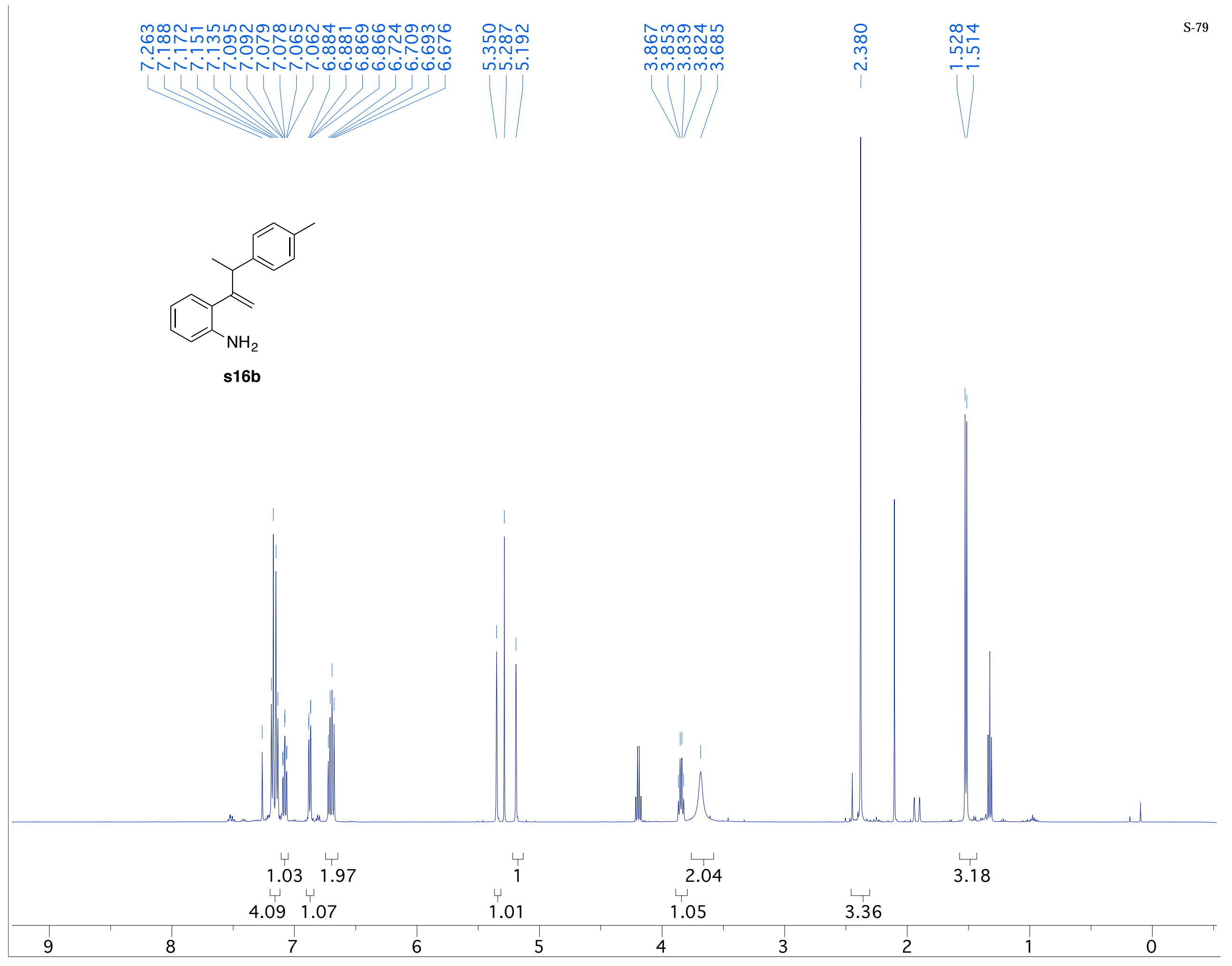




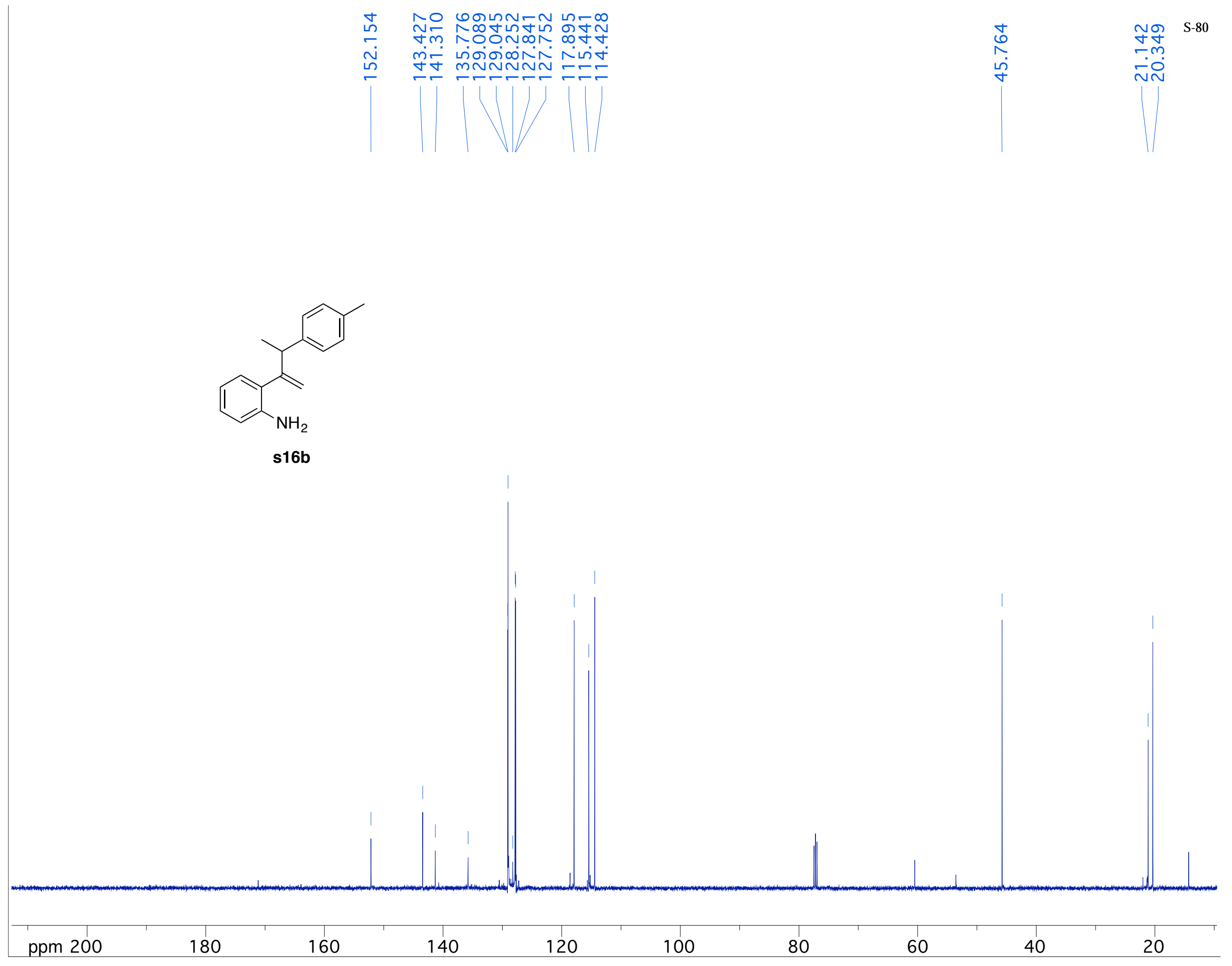




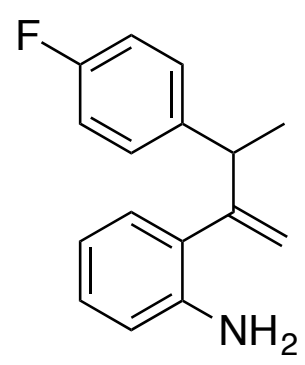

s16c

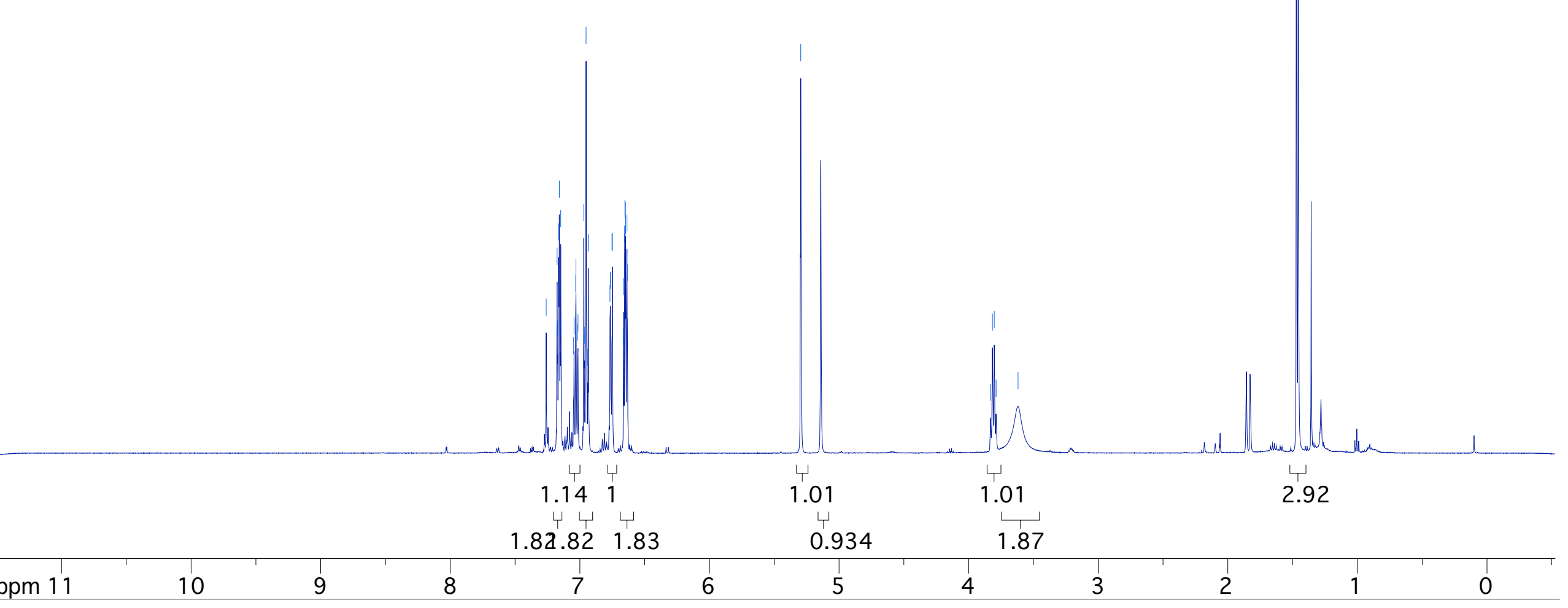




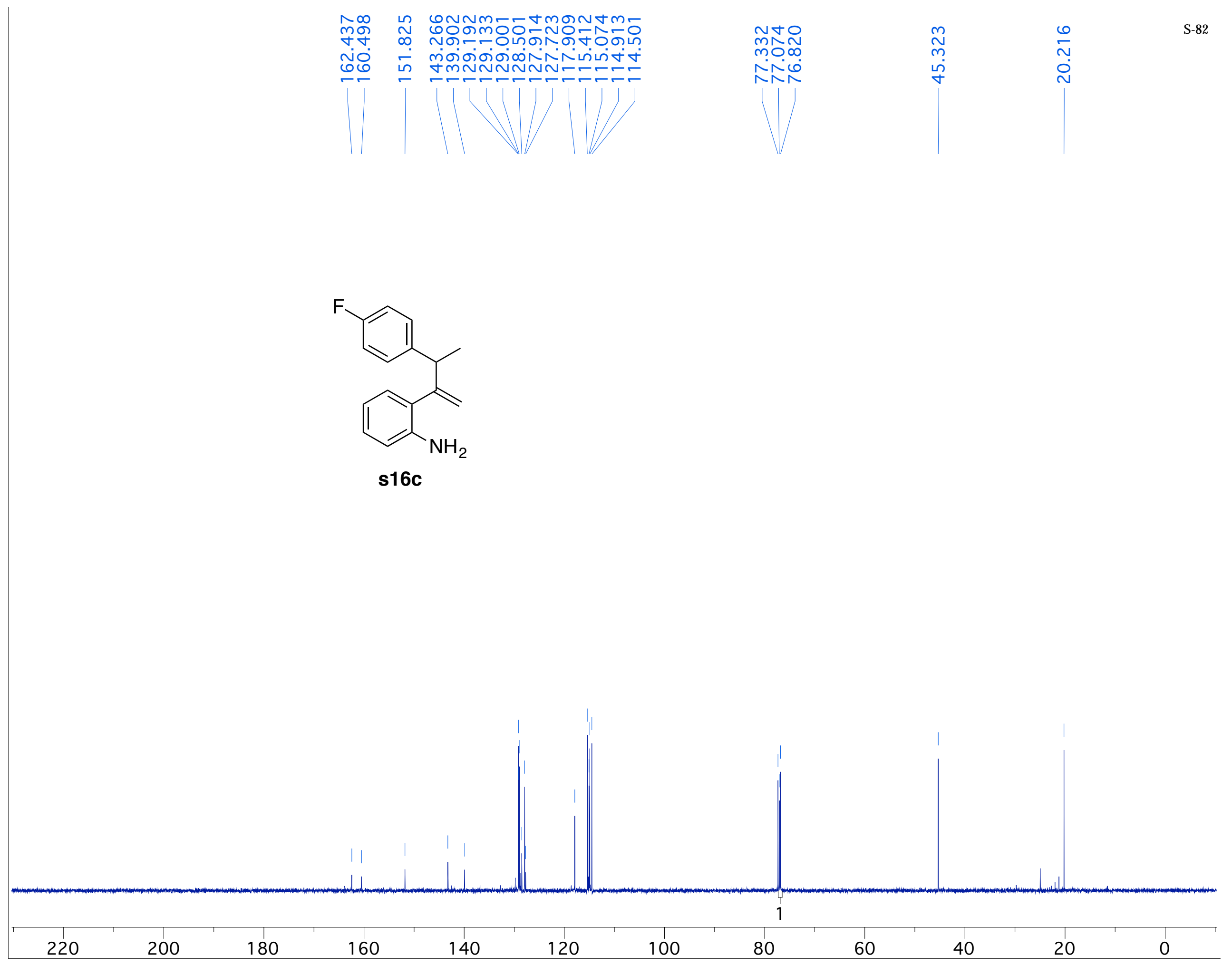




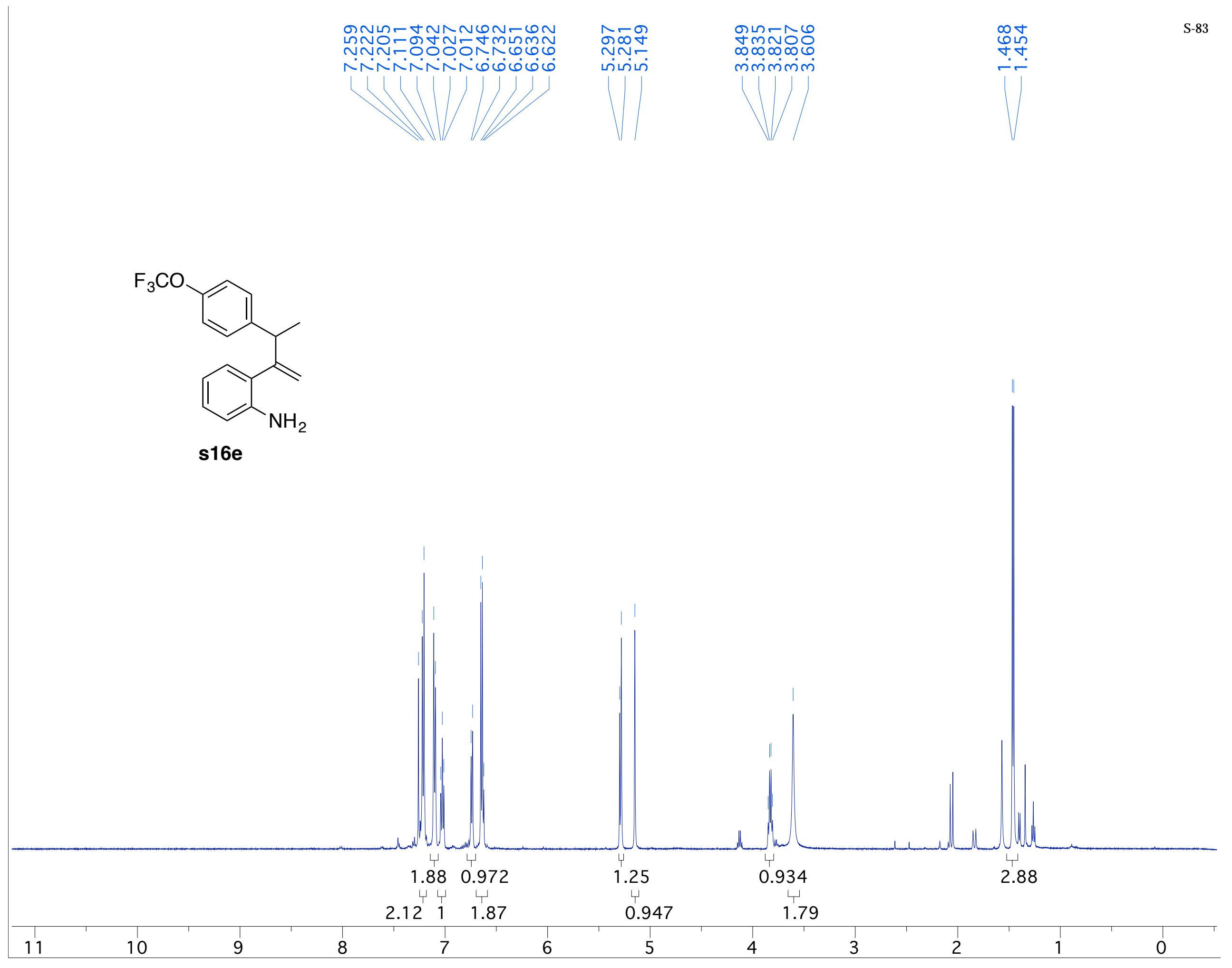




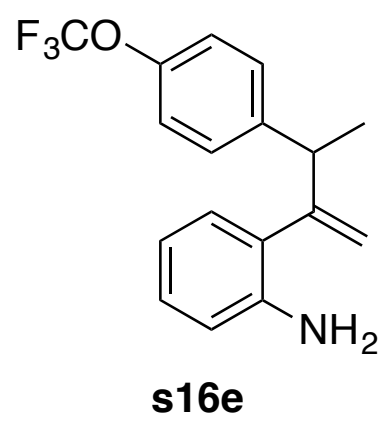

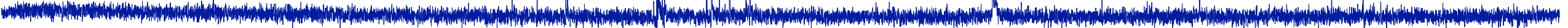




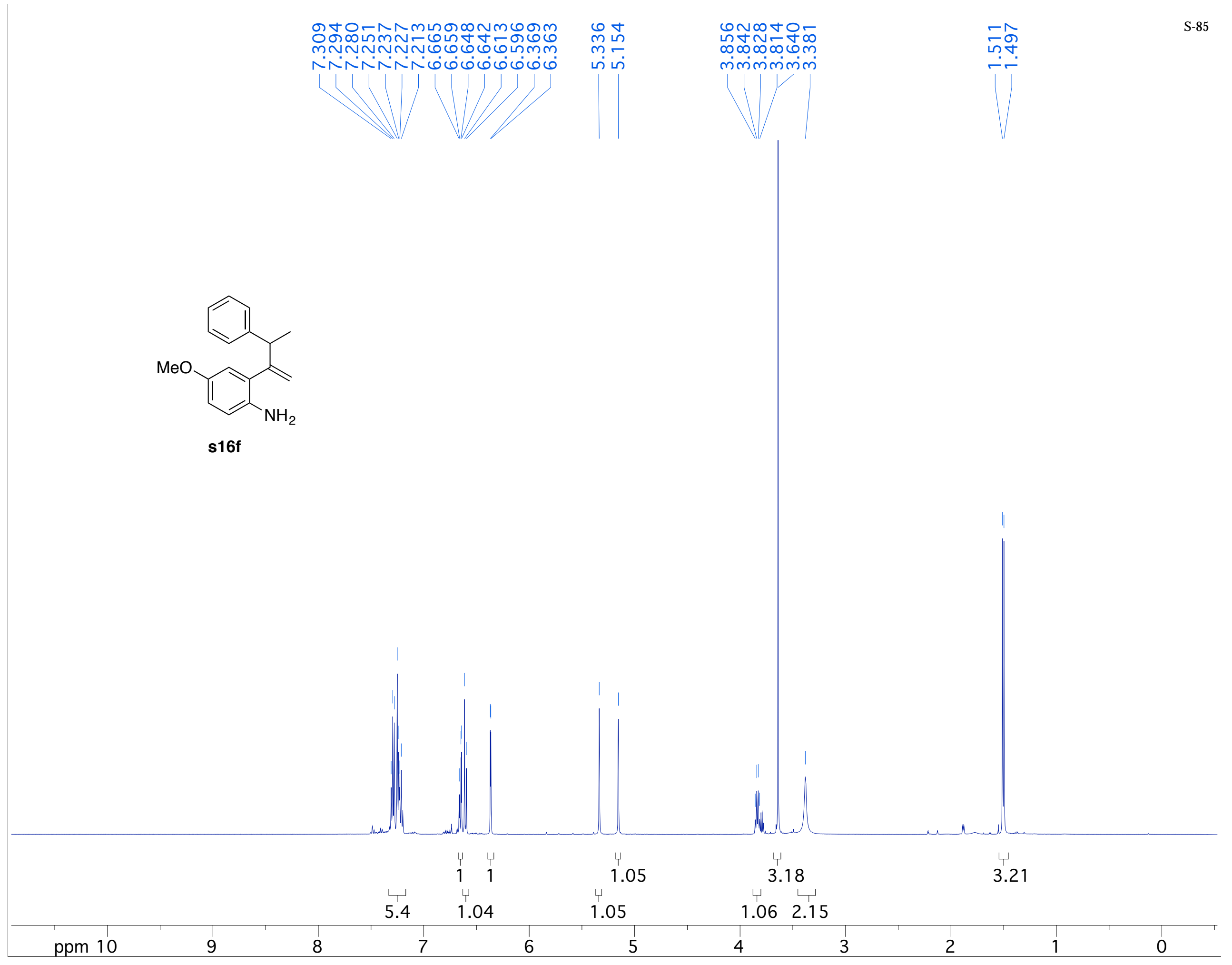




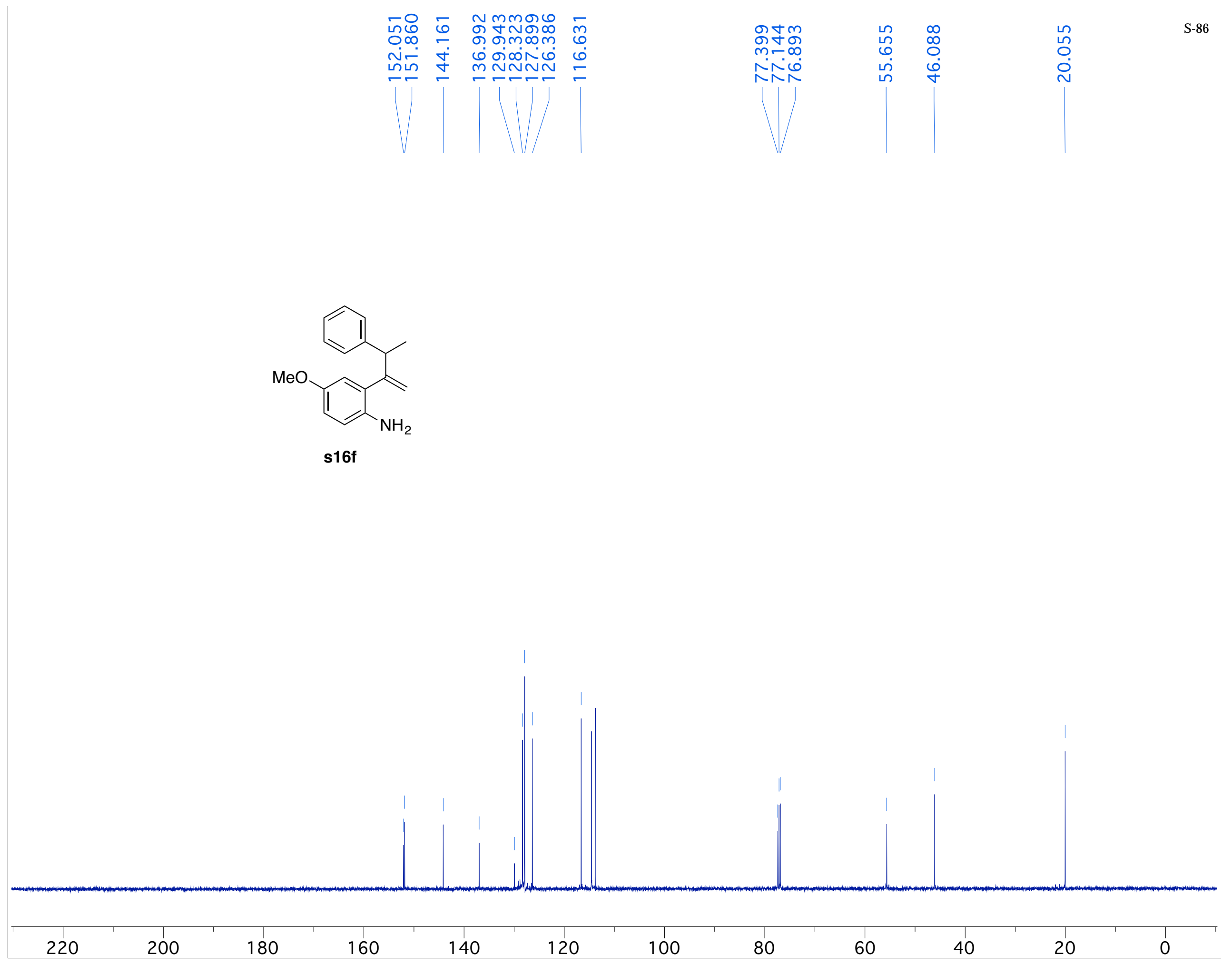




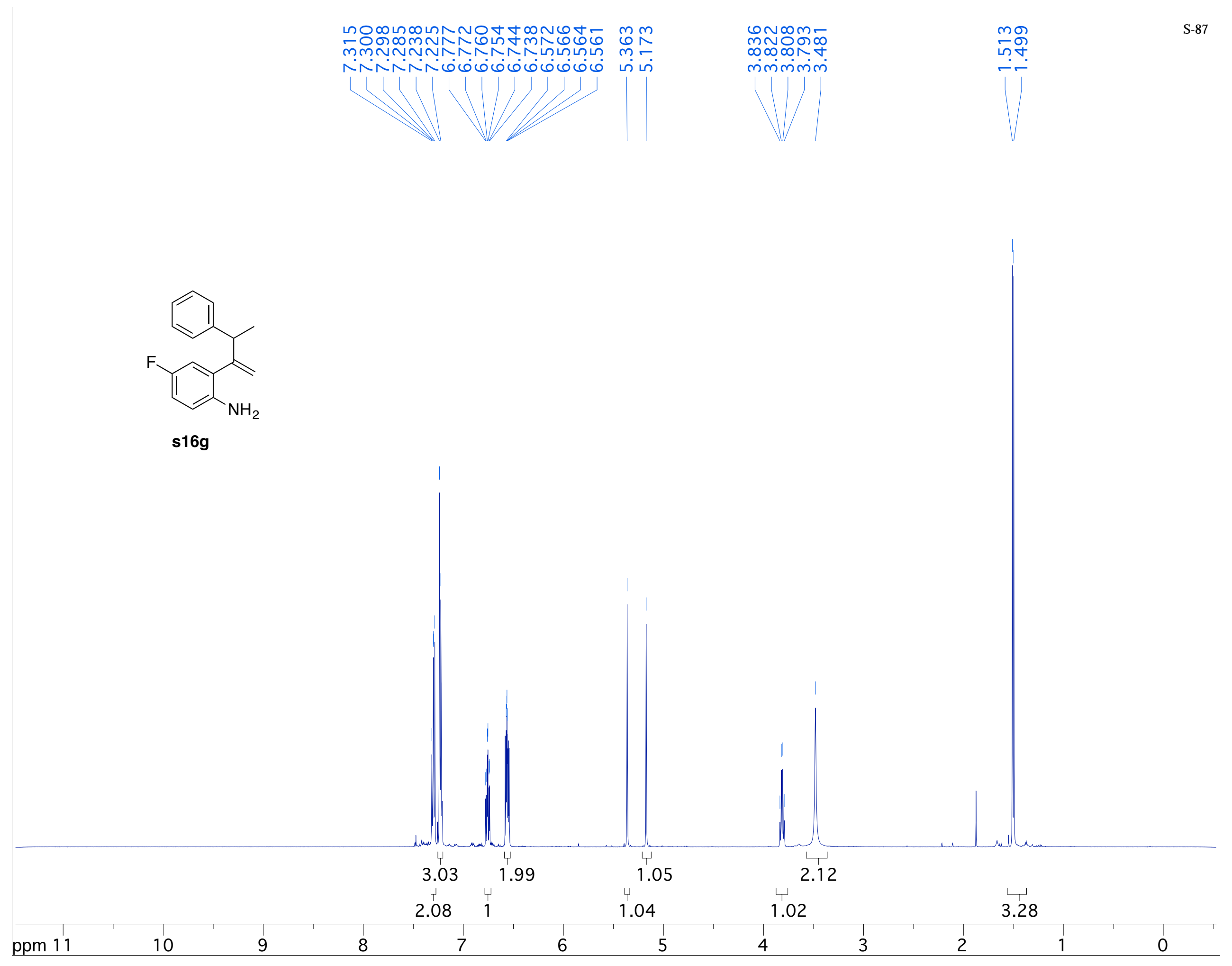




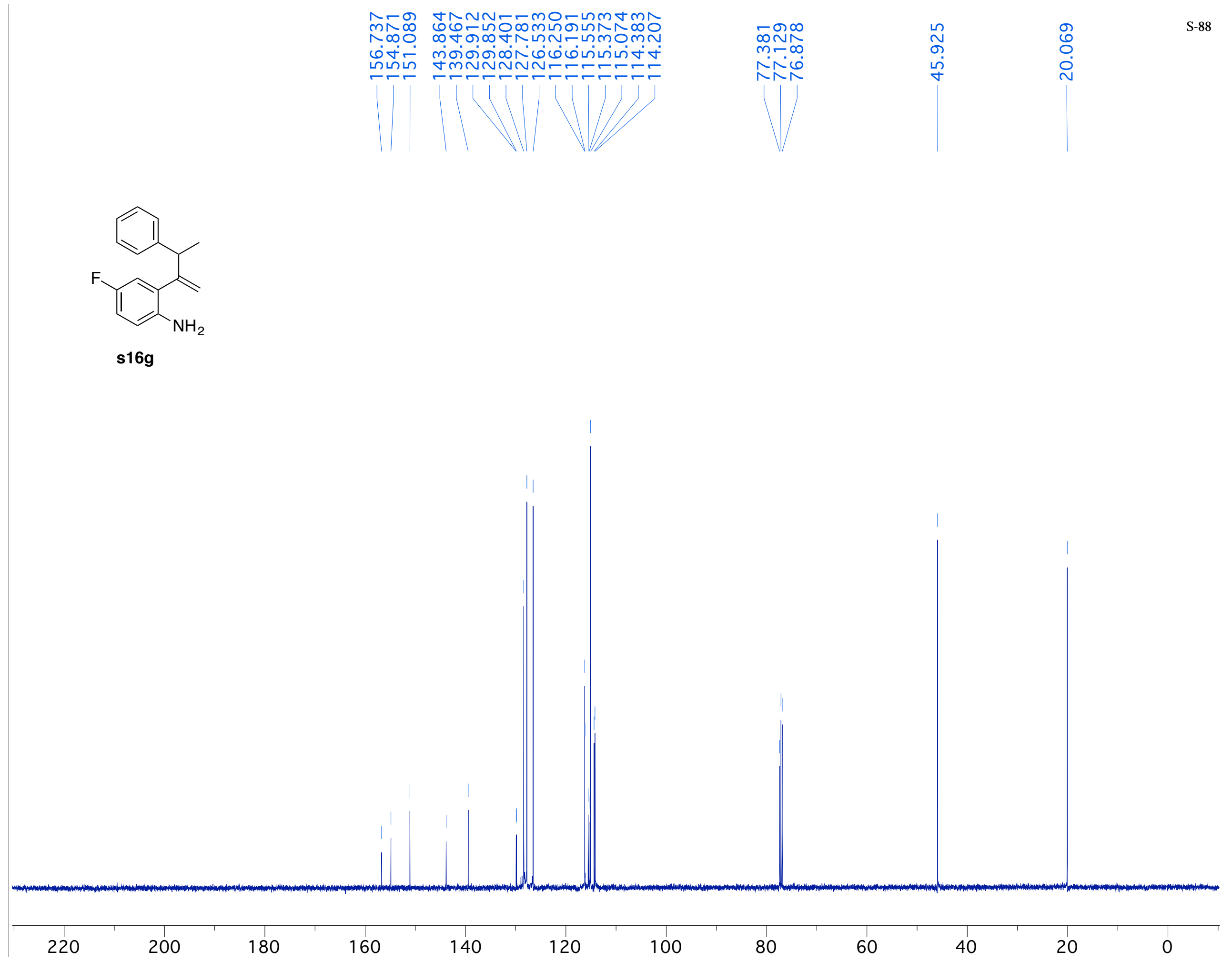




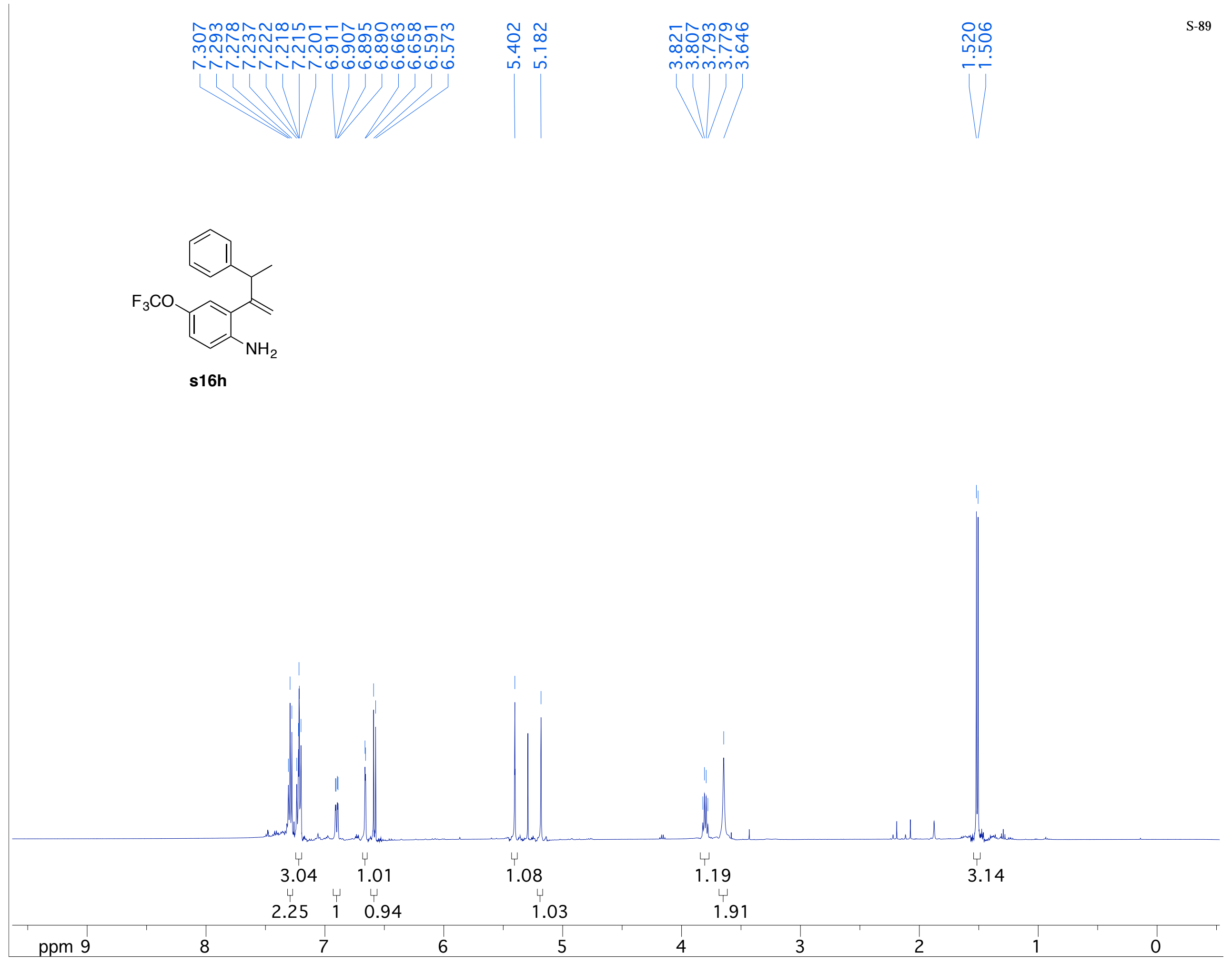




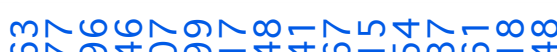

UNG

(min.

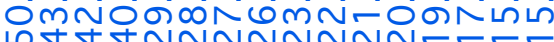

-ーー - - - - -
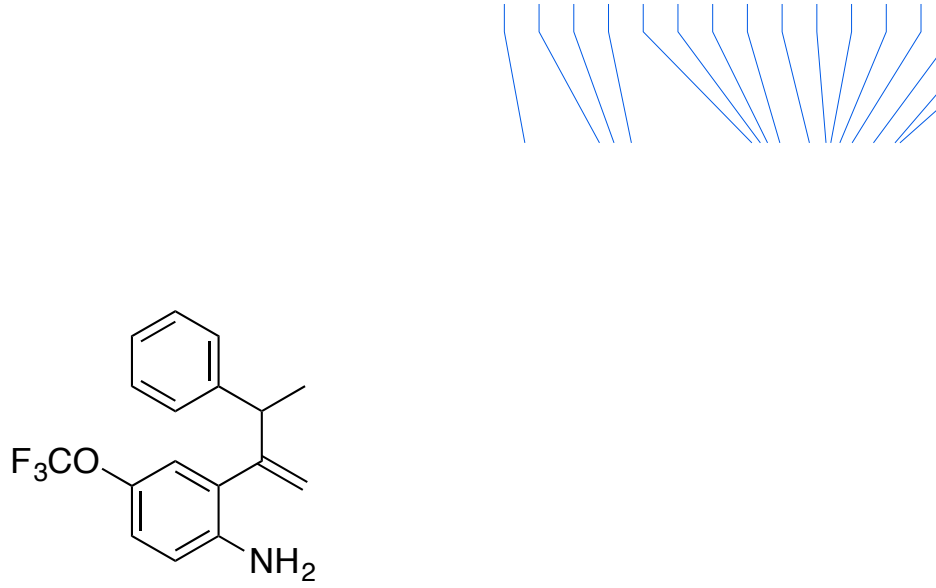

s16h

$\mathrm{NH}_{2}$
ก)

mon

Nín

ก̊

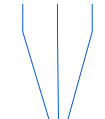

0
$\infty$
$\infty$
0 


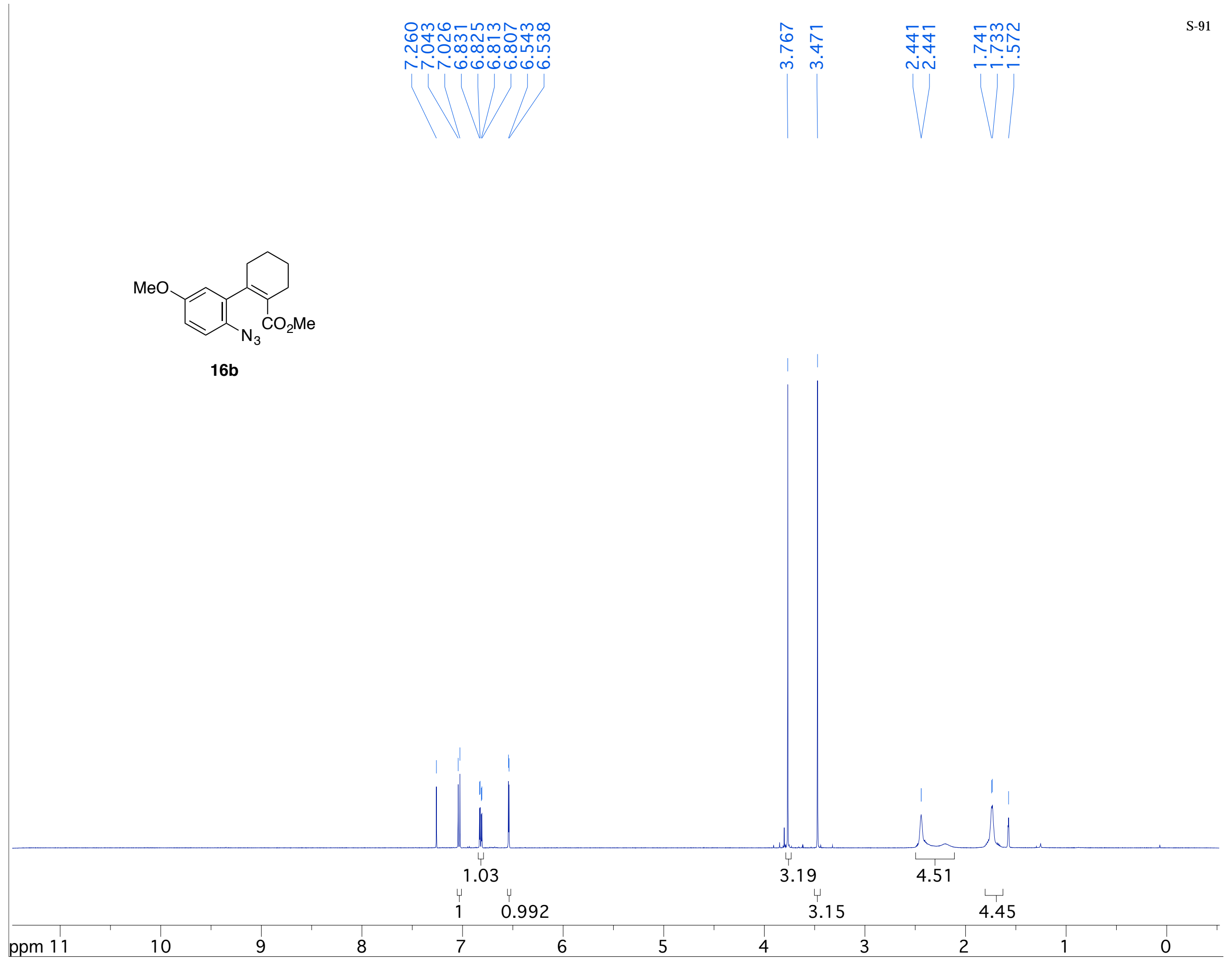



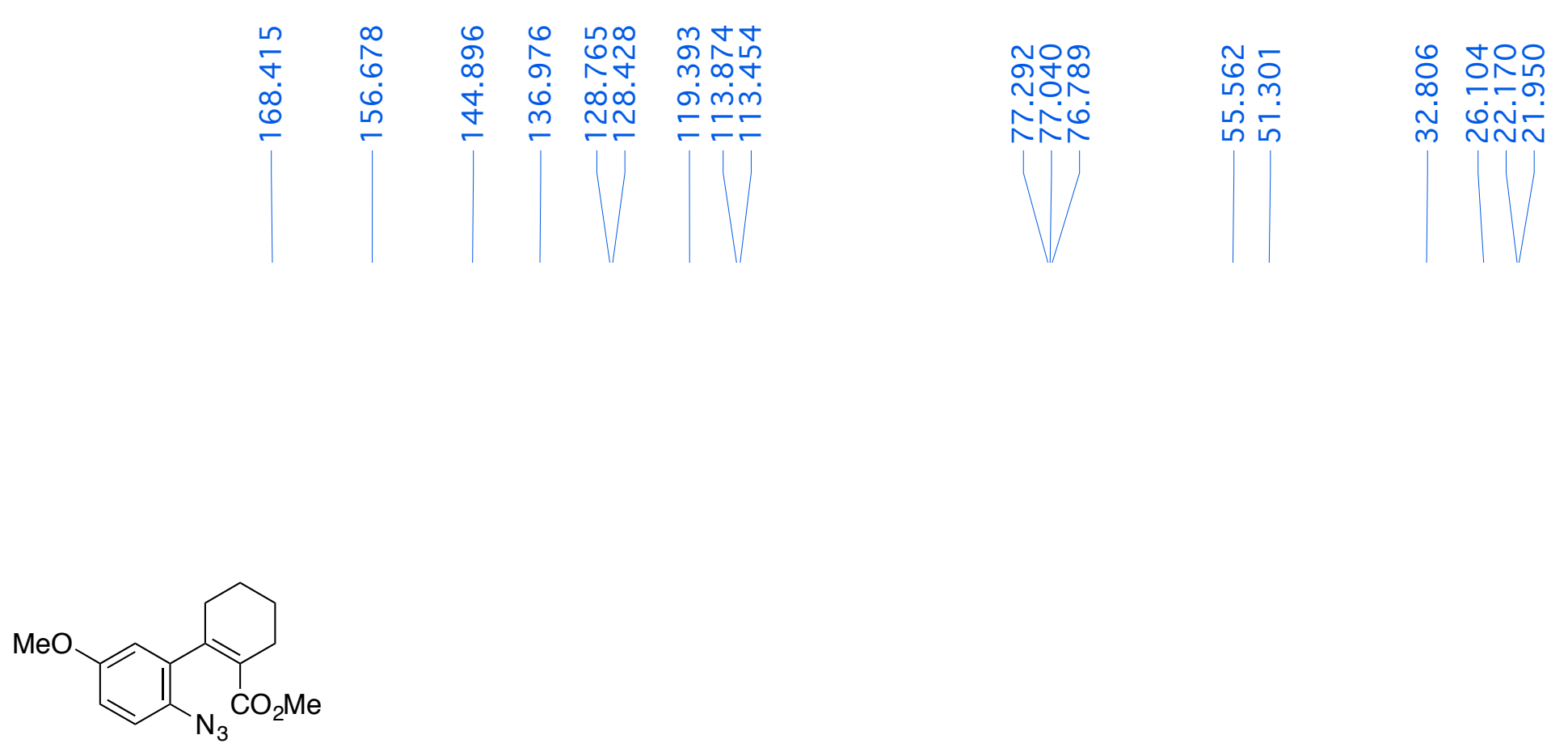

16b

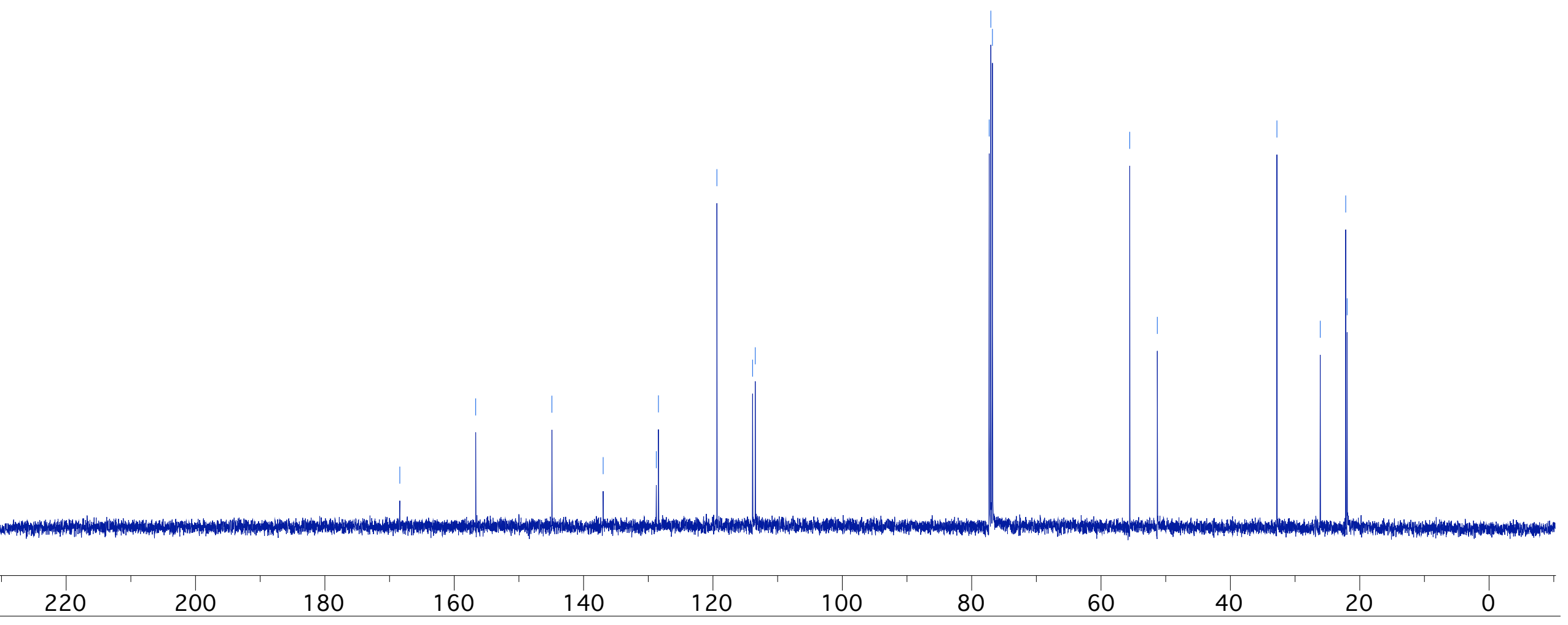




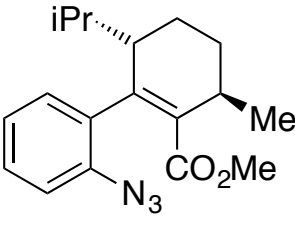

18 (major)

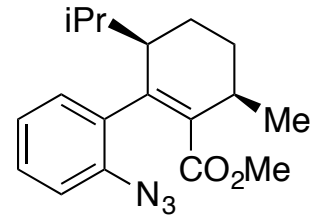

$3: 1$

18' (minor)

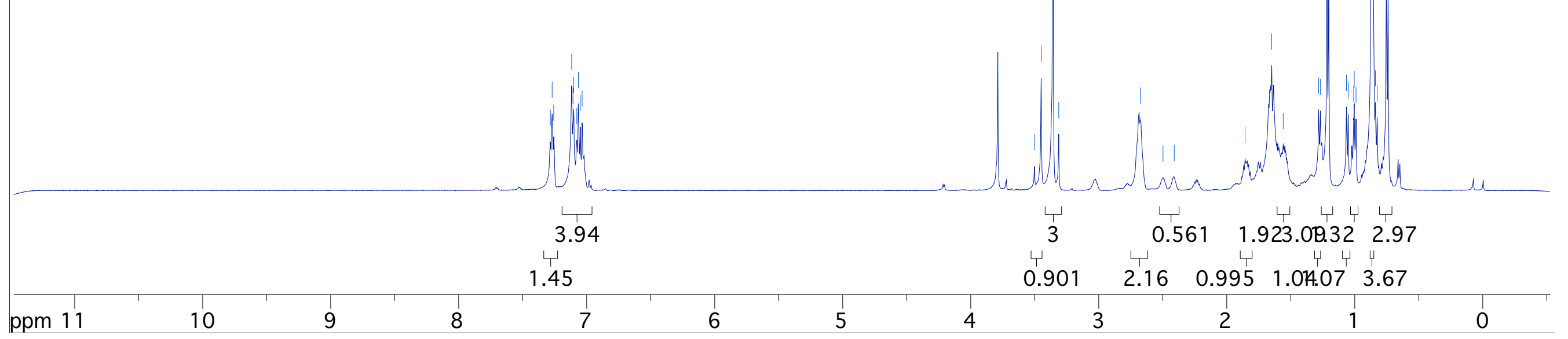




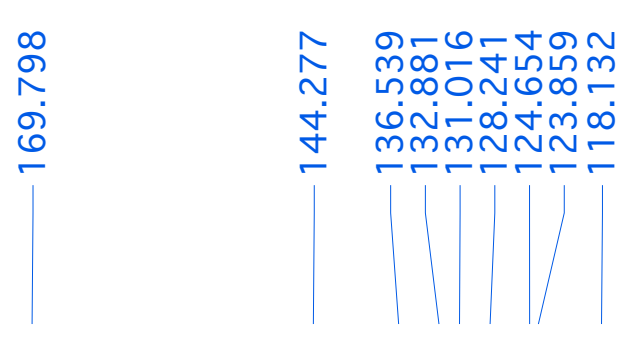

애워

Nंن

నٓ⿵

-0 0.

ทีธ

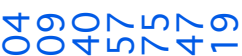

on-mó

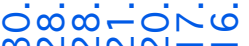

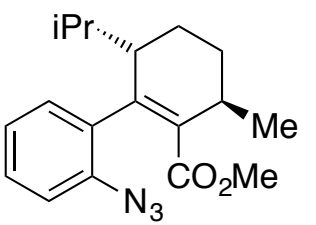

18 (major)

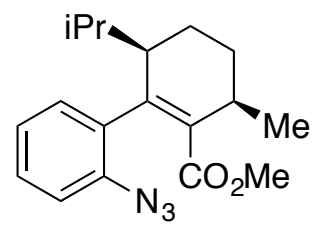

$3: 1$

18' (minor) 


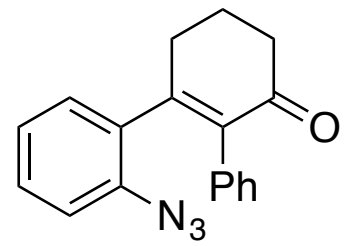

24a

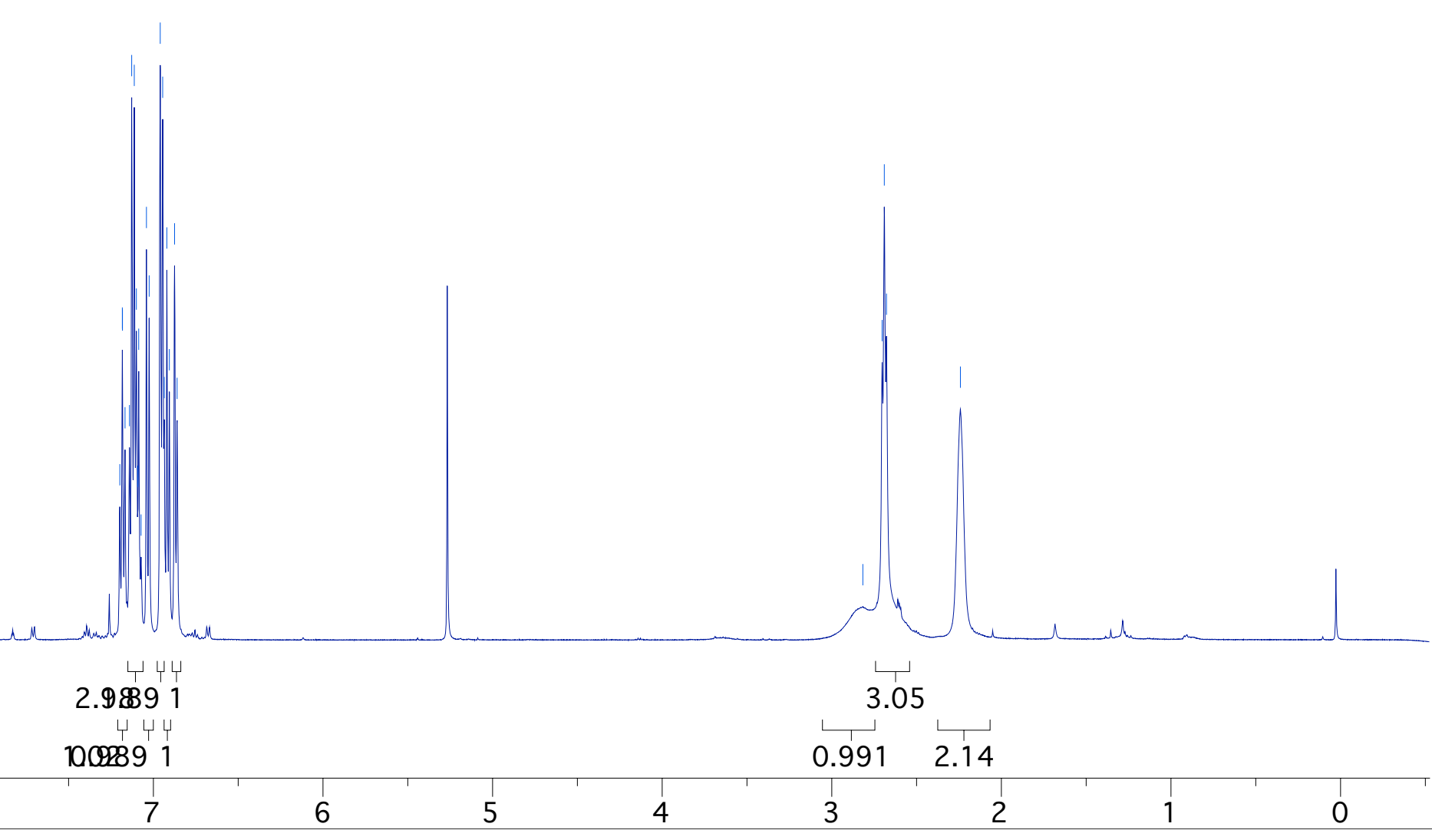




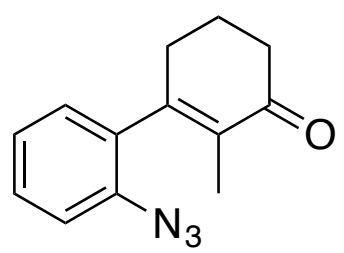

$24 b$

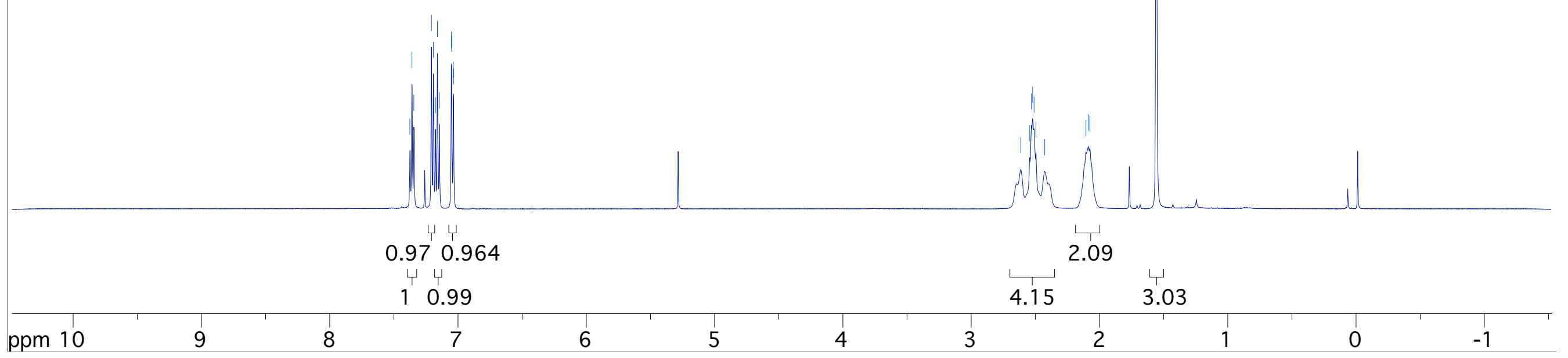


<smiles>CC1=C(c2ccccc2N)CCCC1=O</smiles>

24b

\section{b}




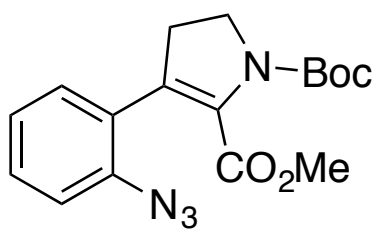

24d

wh the the

11.02

1.04 


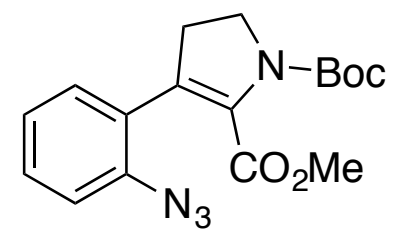

24d 


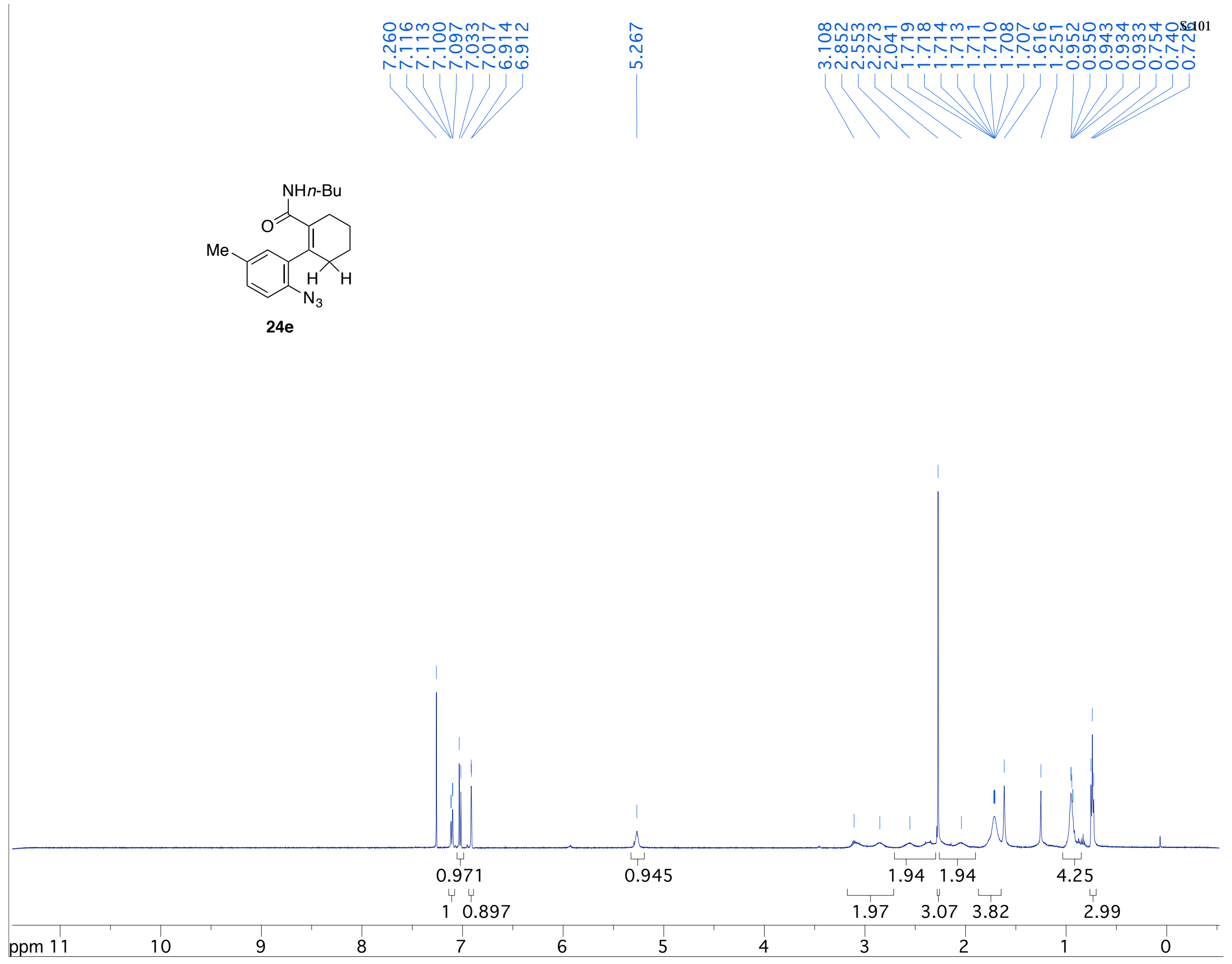




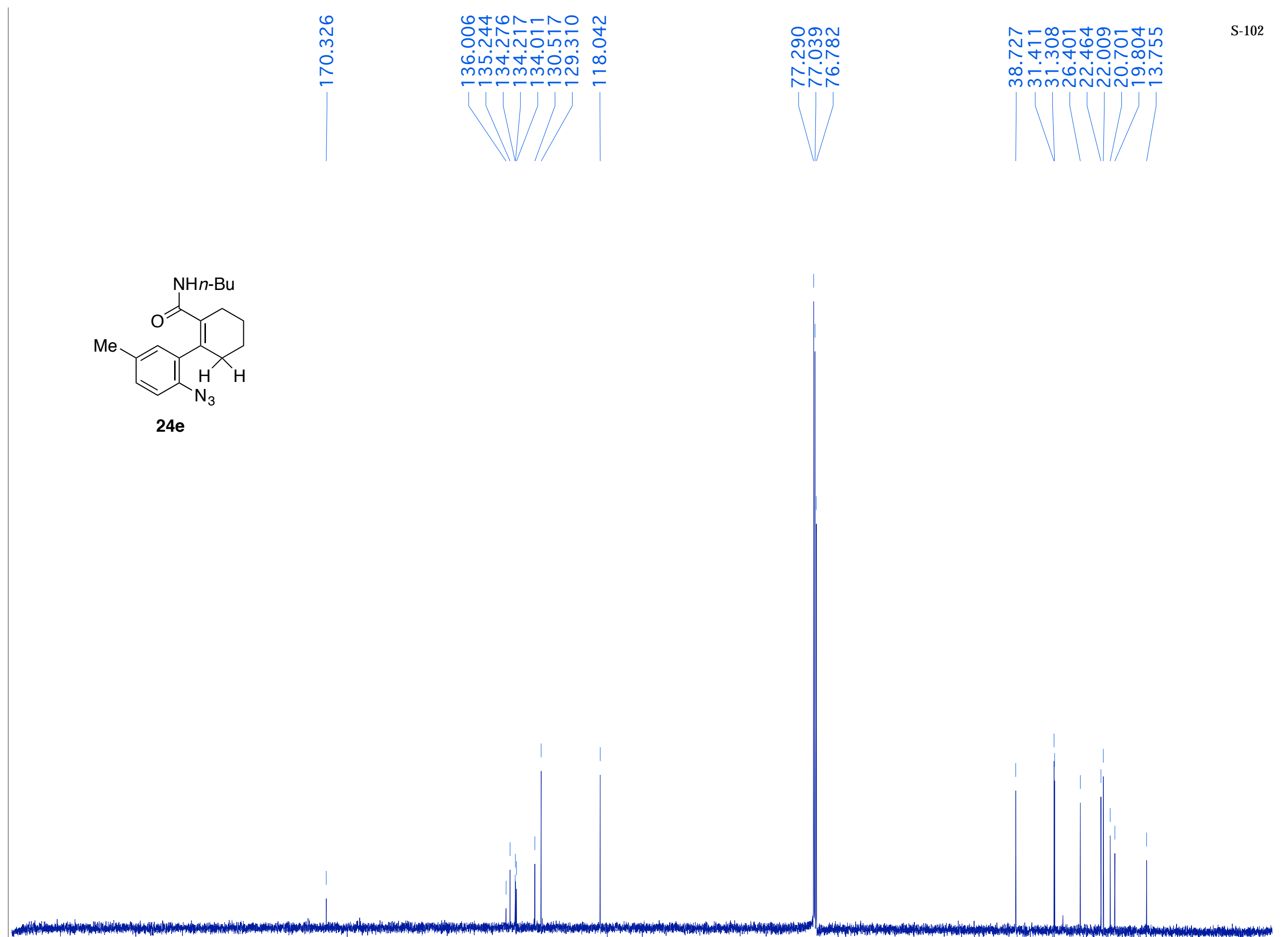

\begin{tabular}{|c|c|c|c|c|c|c|c|c|c|c|c|}
\hline 220 & 200 & 180 & 160 & 140 & 120 & 100 & 80 & 60 & 40 & 20 & 0 \\
\hline
\end{tabular}




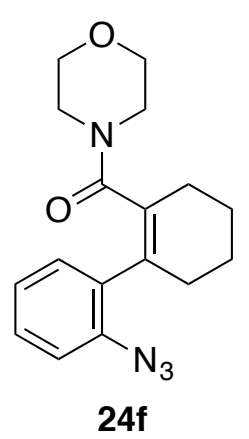

$24 f$

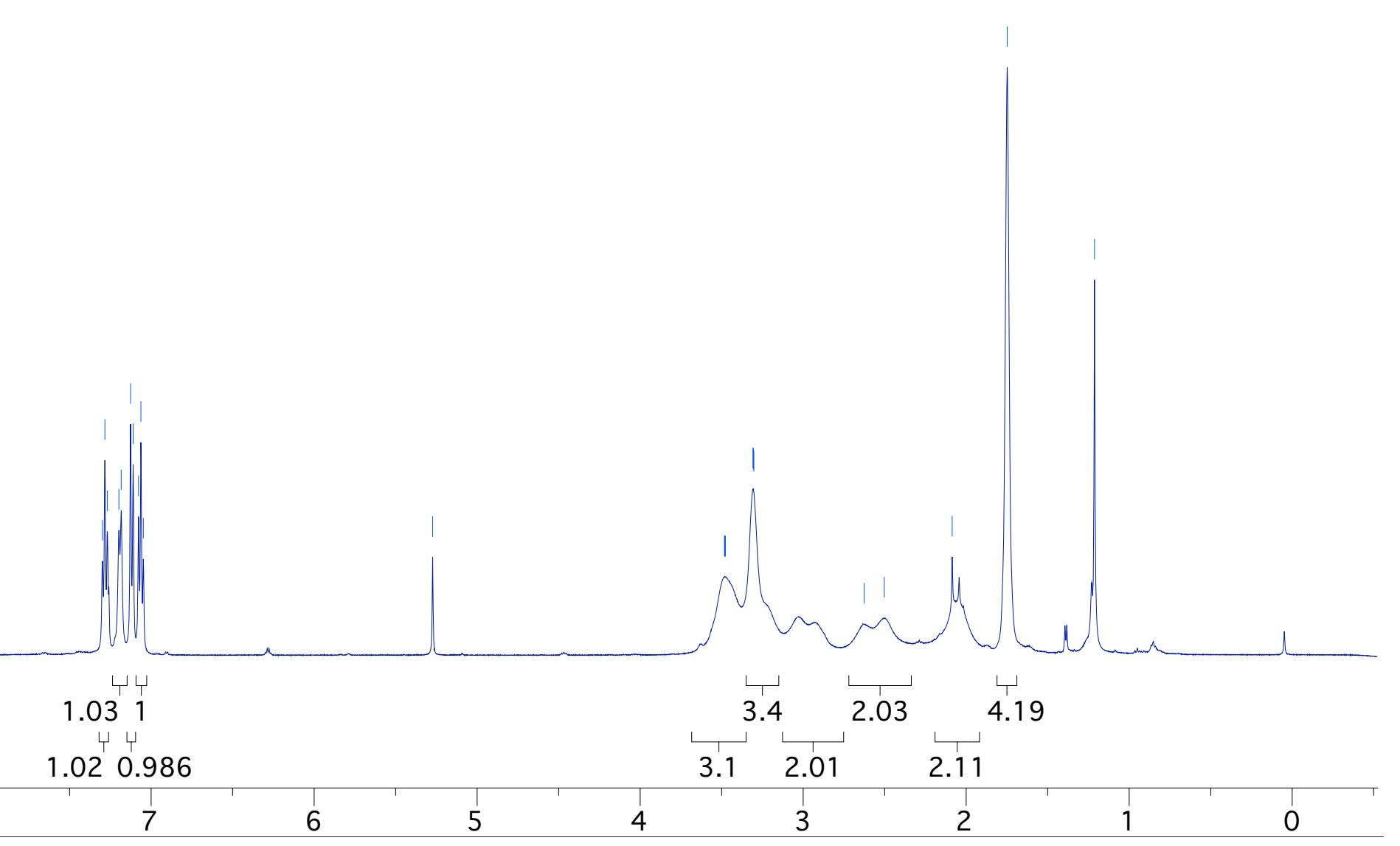



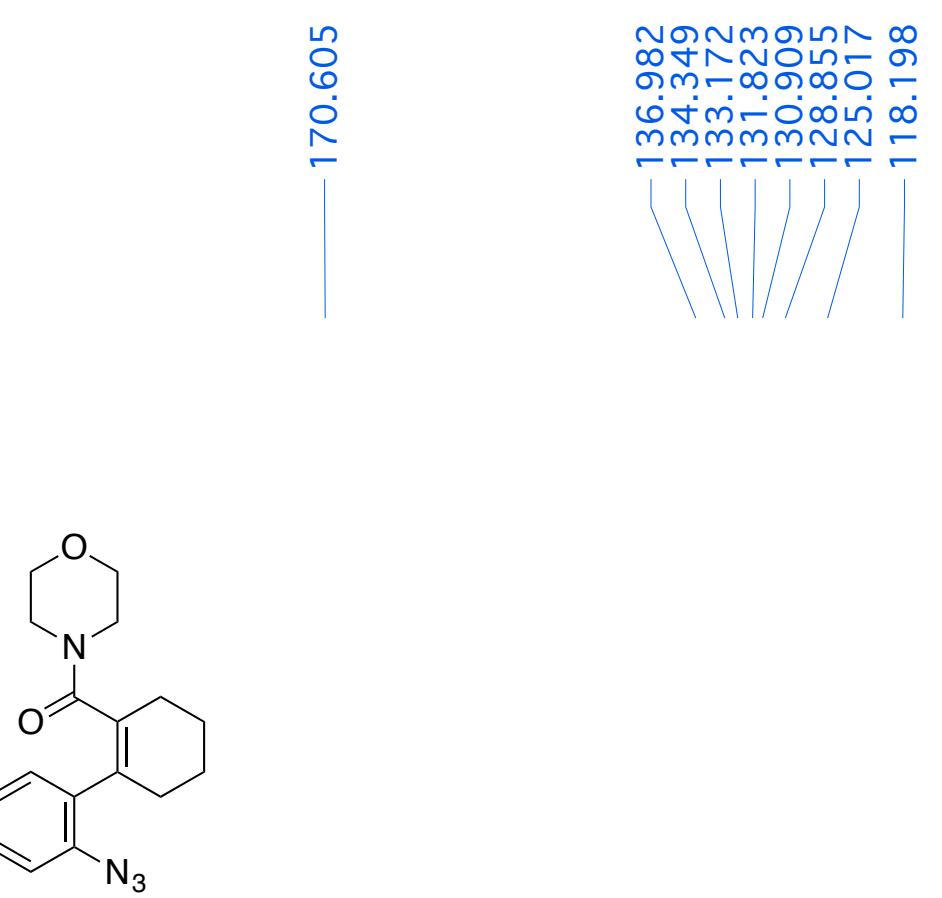

$24 f$

\section{$4 f$}




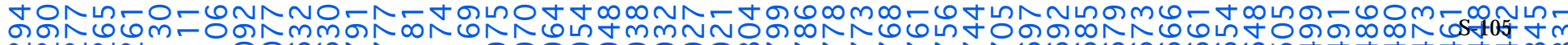

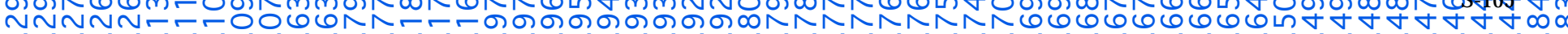

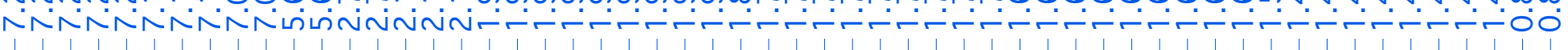<smiles>CC1CCCC=C1c1ccccc1N</smiles>

27

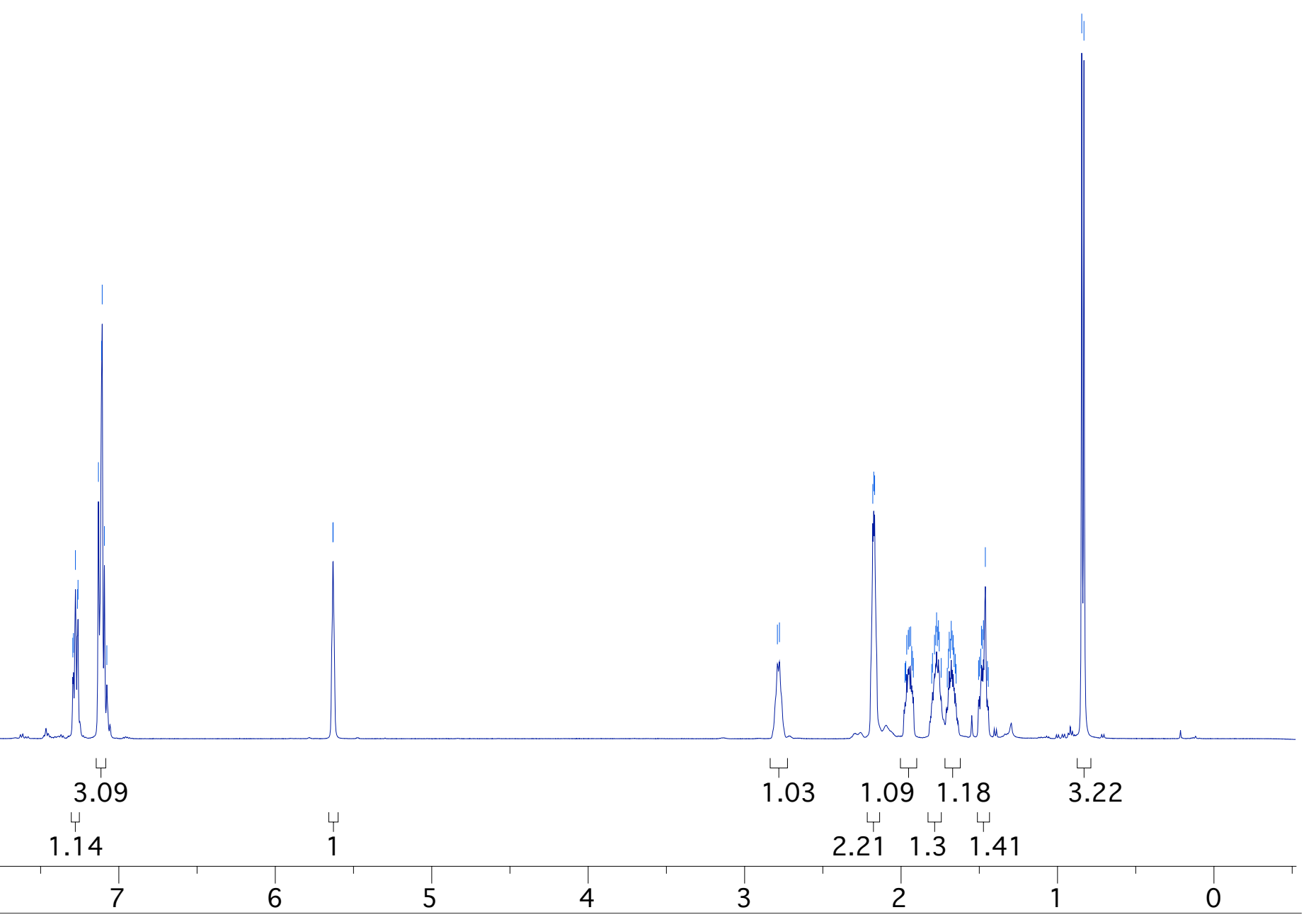




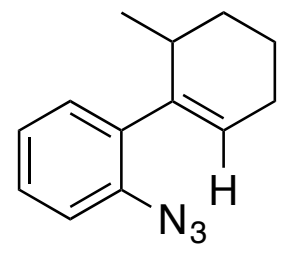

27 


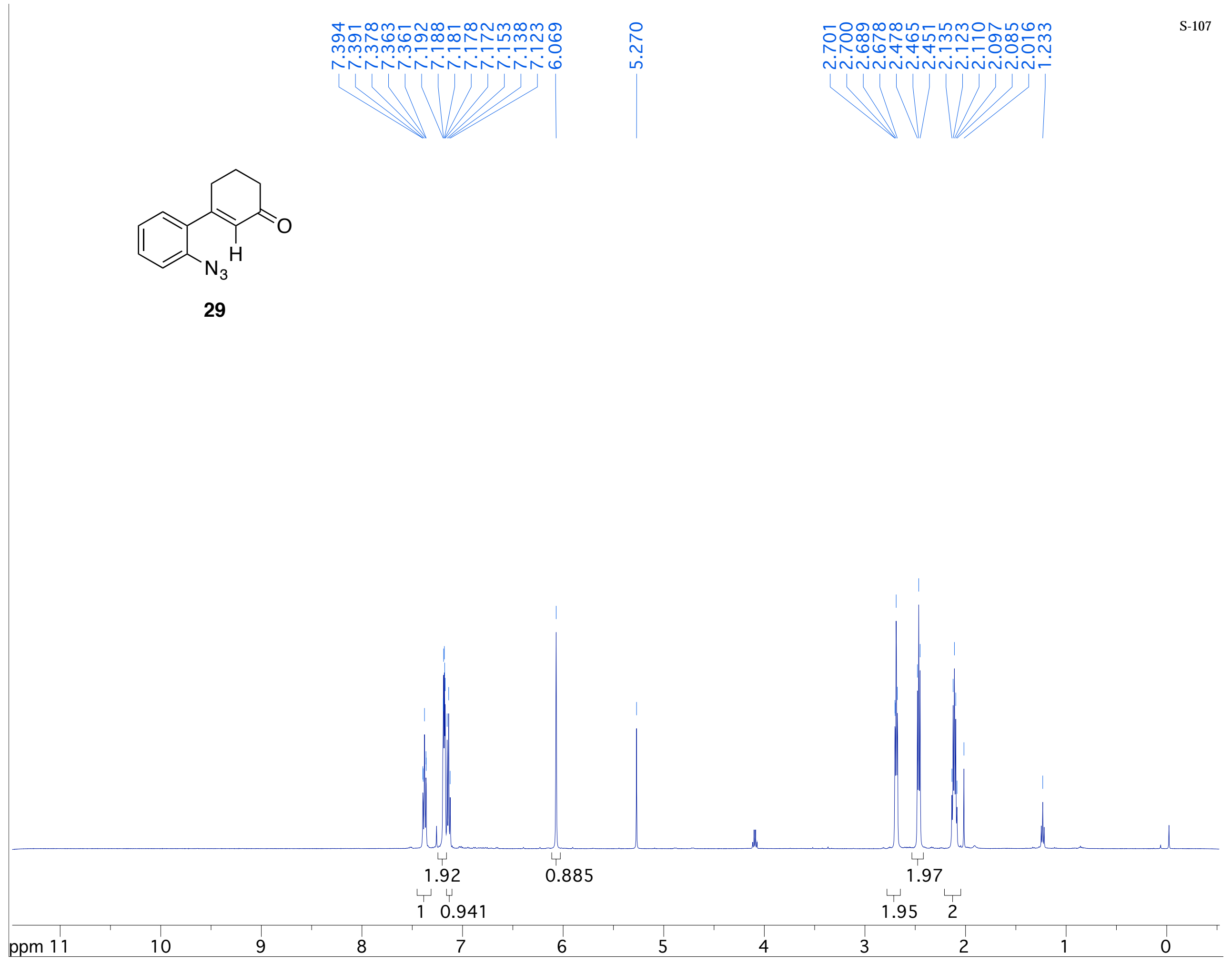




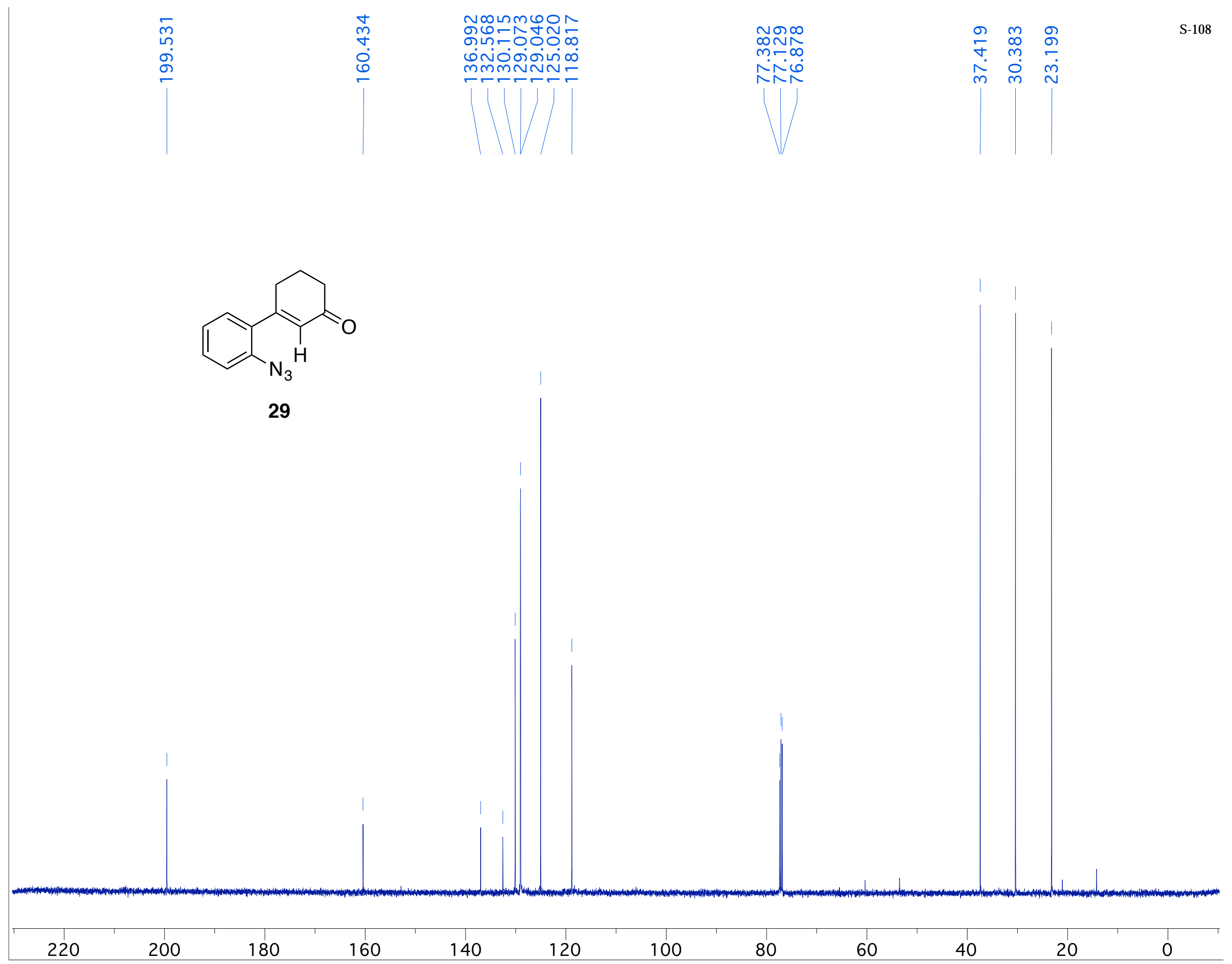




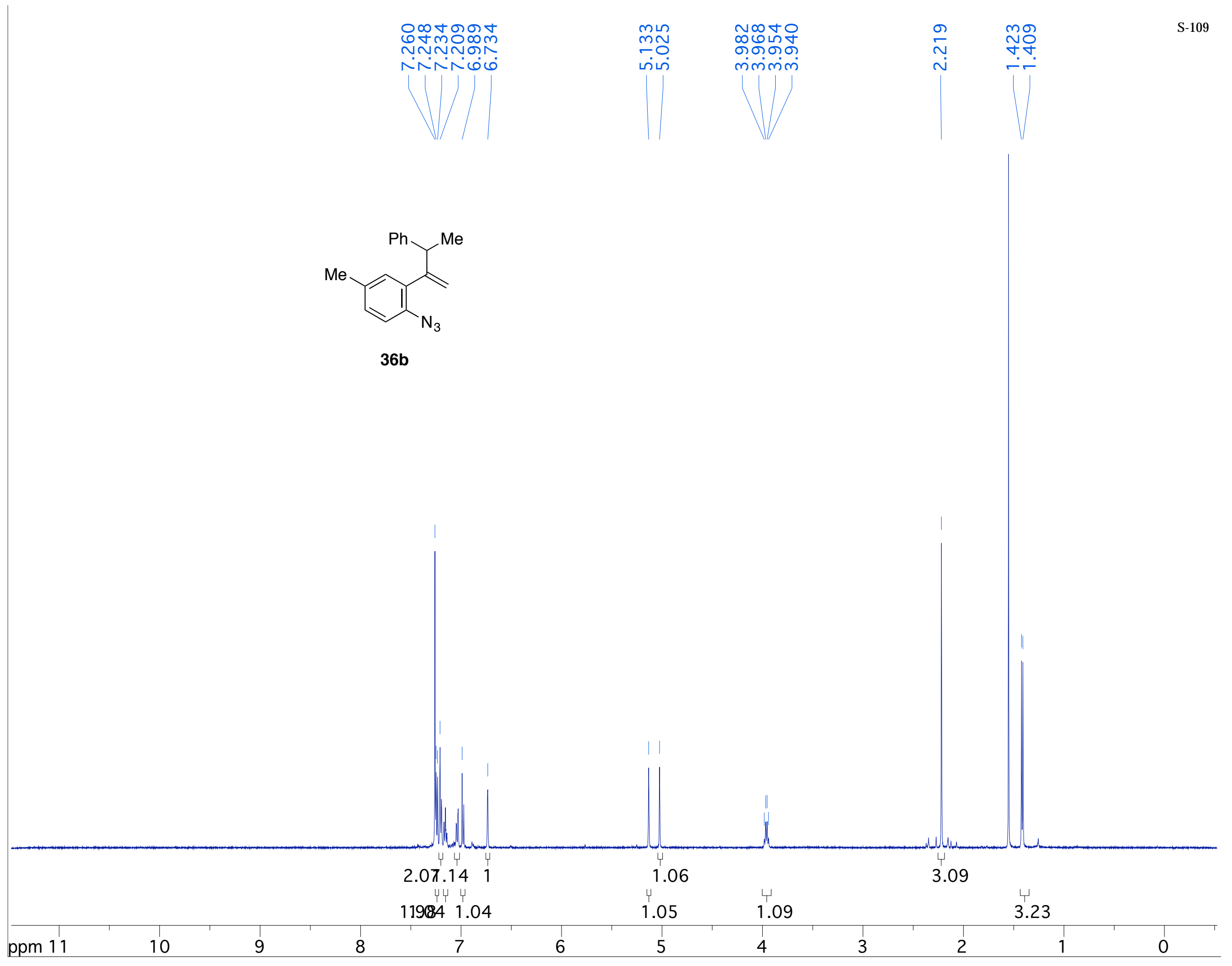




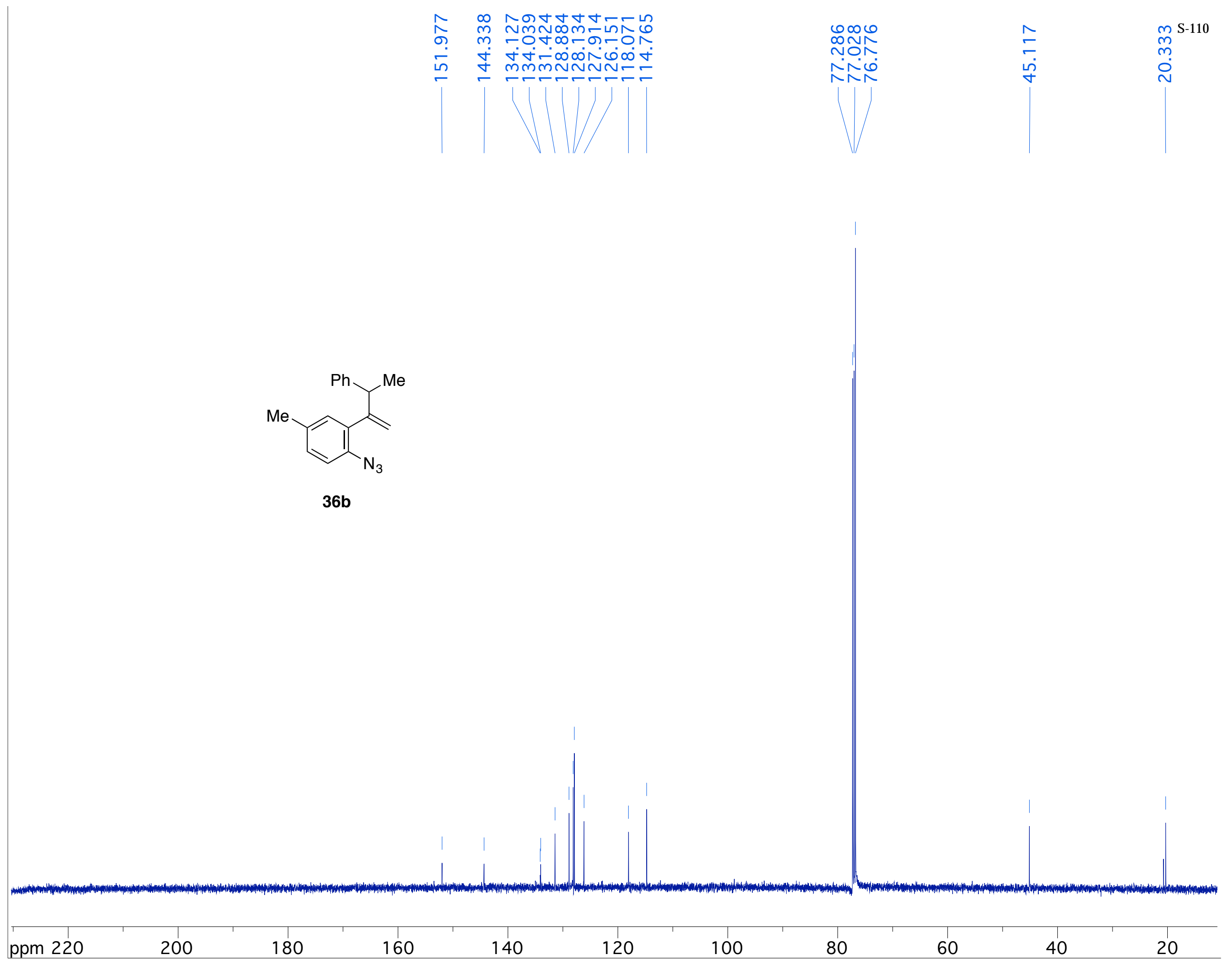




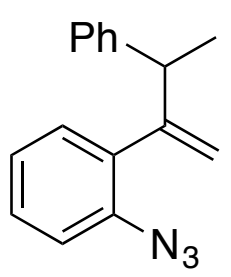

$36 c$

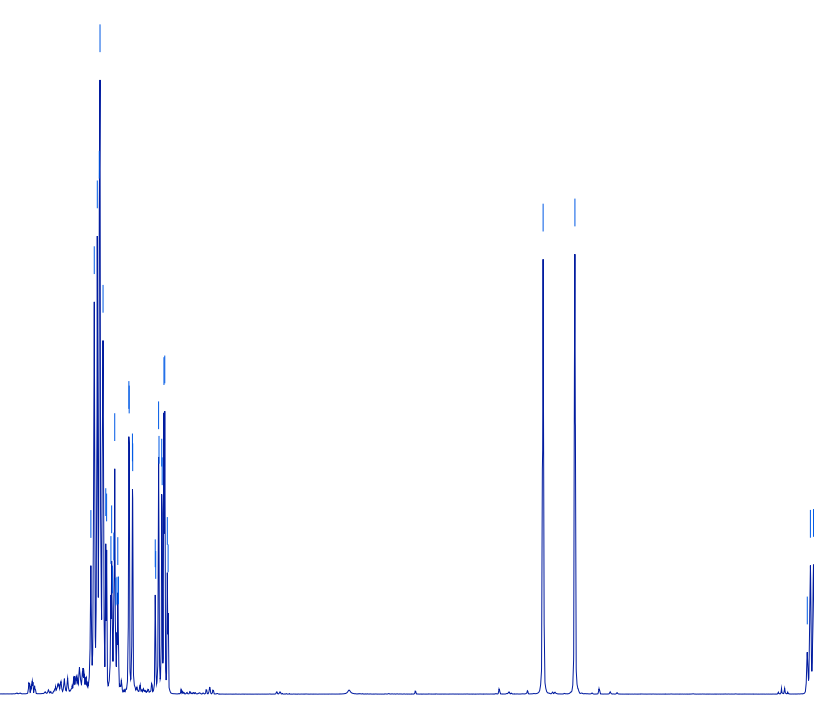

1.22 .03

1.01

5.251 .02

1.01

3.06

5



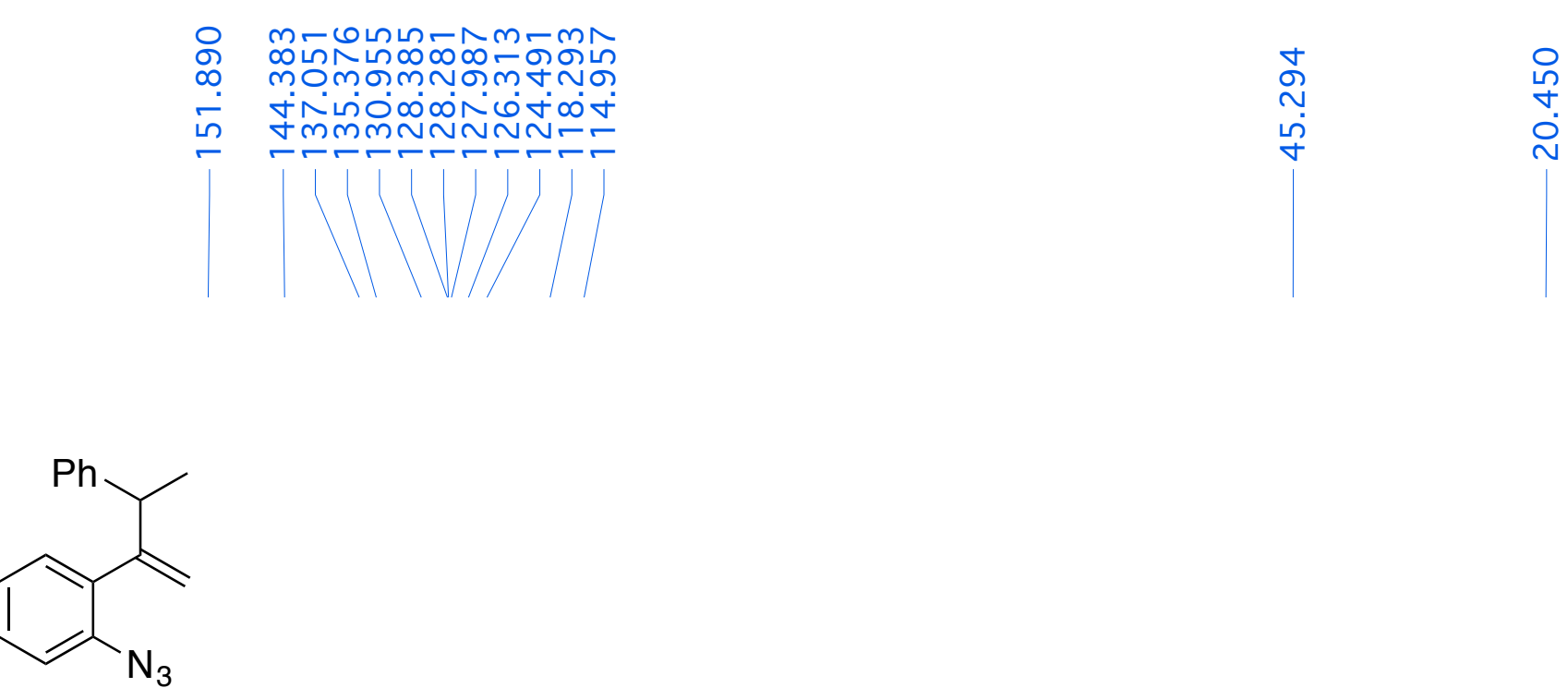

36c 


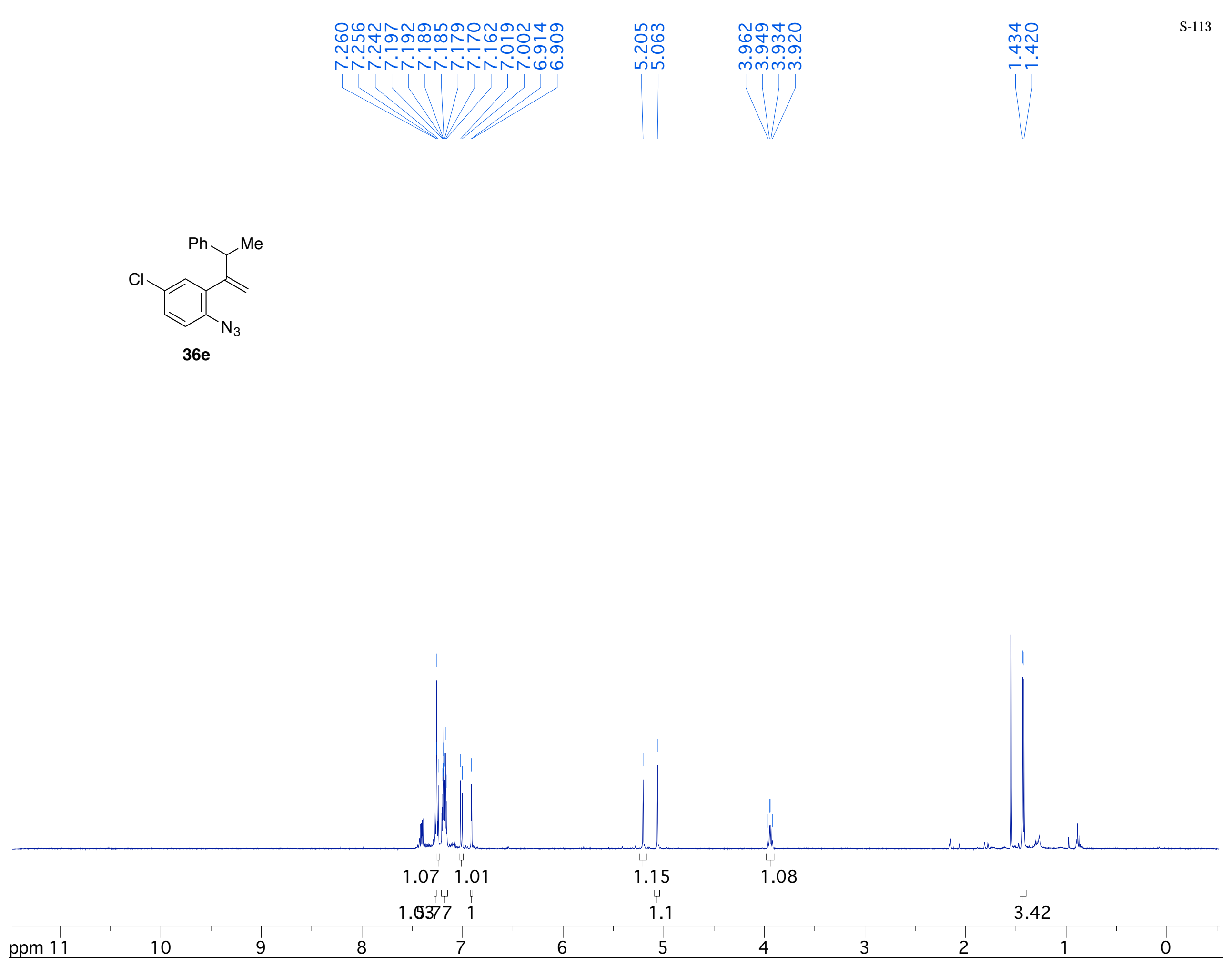


<smiles>C=C(c1cc(Cl)ccc1N=[N+]=[N-])C(C)c1ccccc1</smiles>

$36 \mathrm{e}$ 


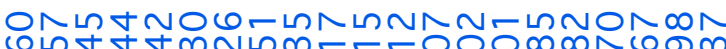
mmmmmmnm-

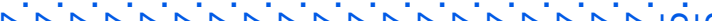

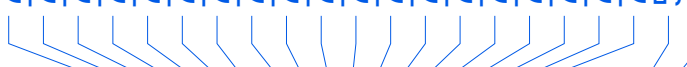

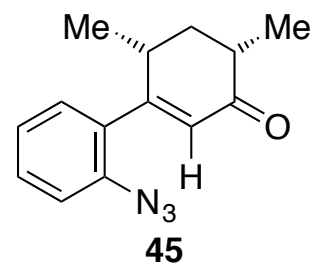

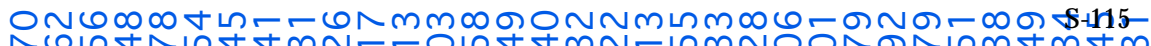
N

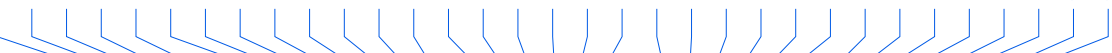




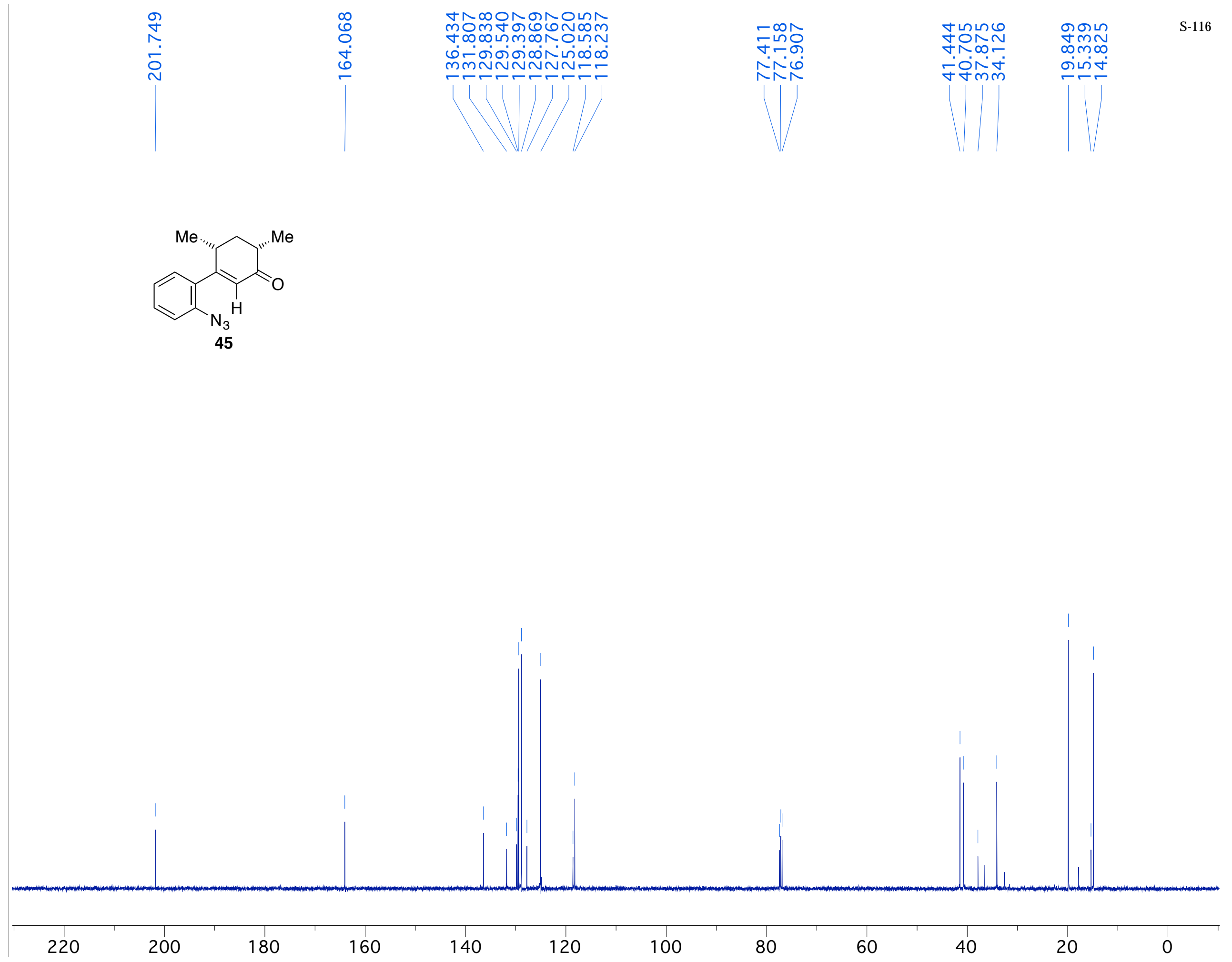




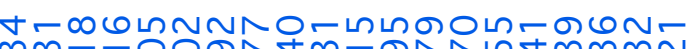

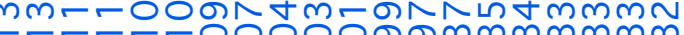

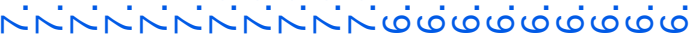

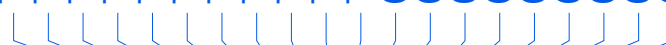
L L

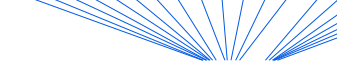<smiles></smiles>

55

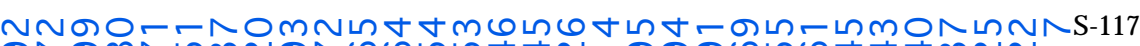
N

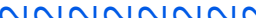
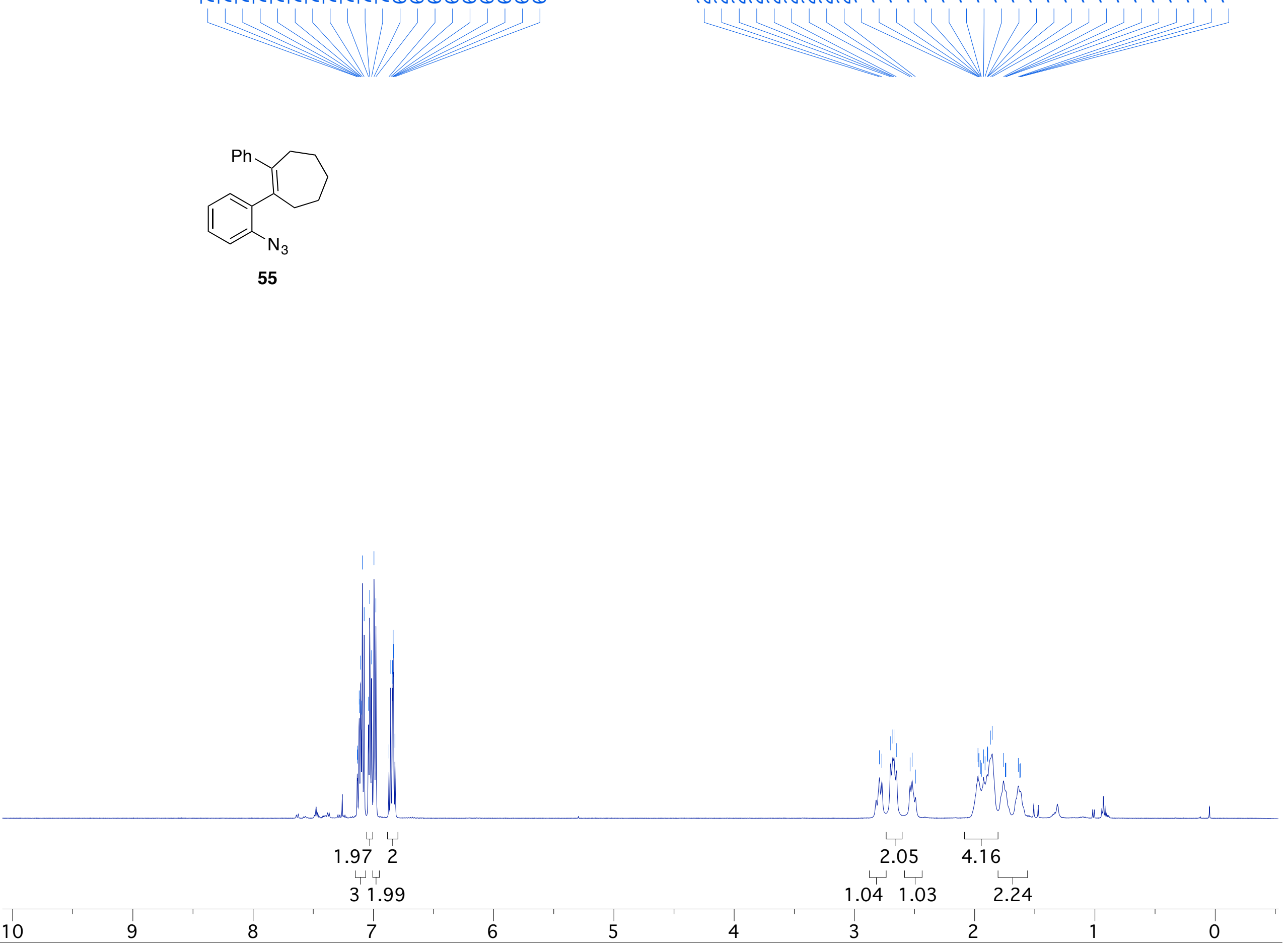


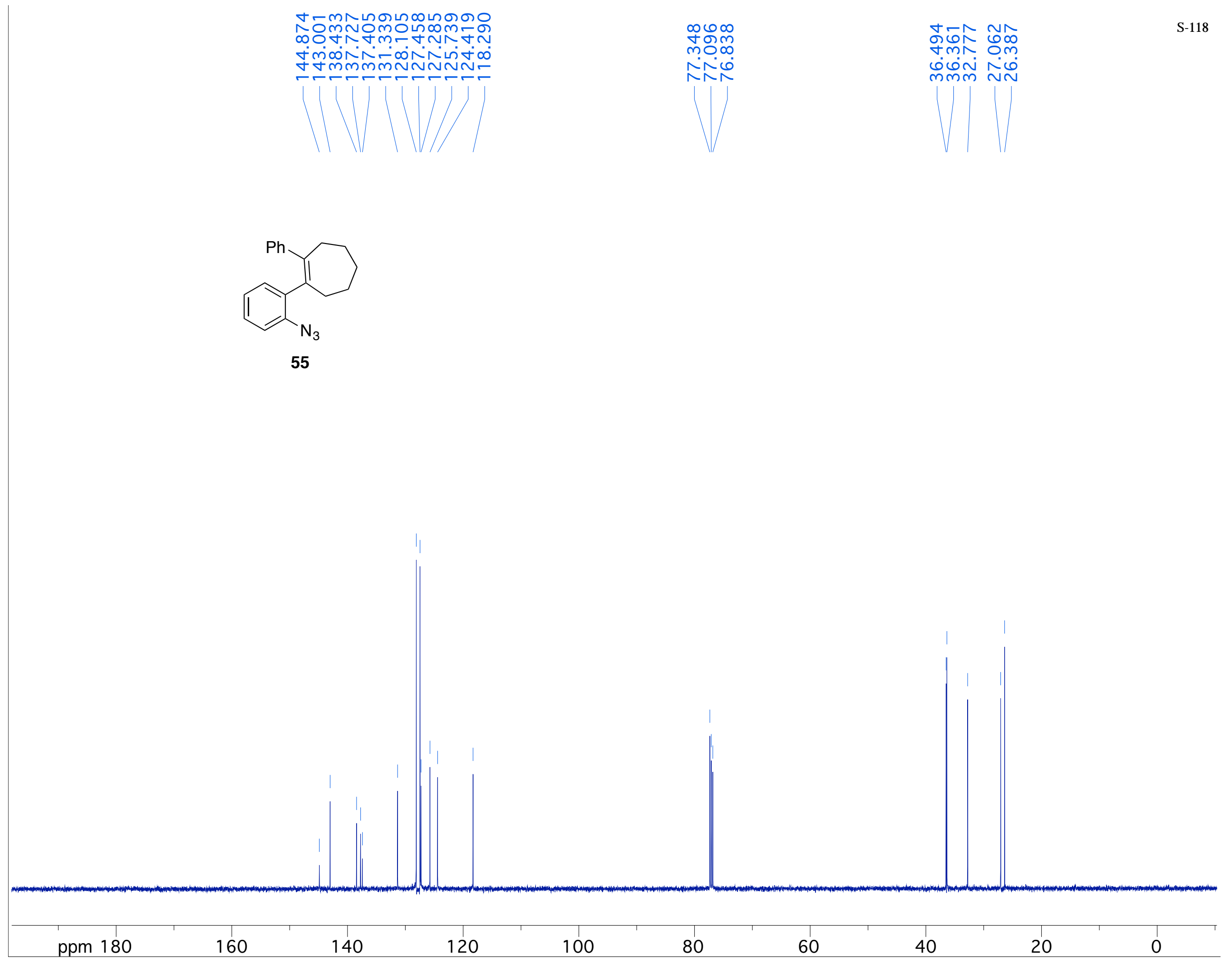




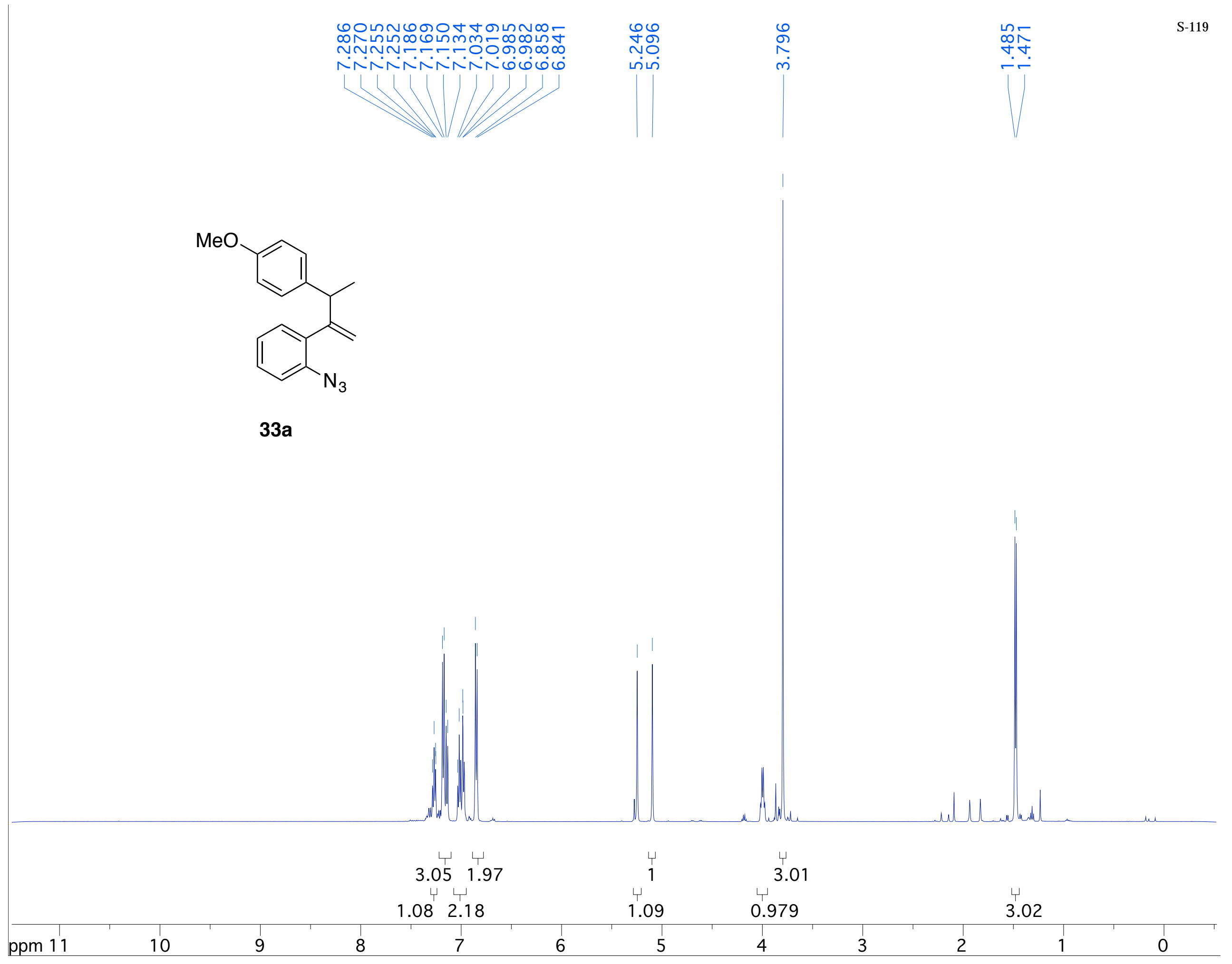




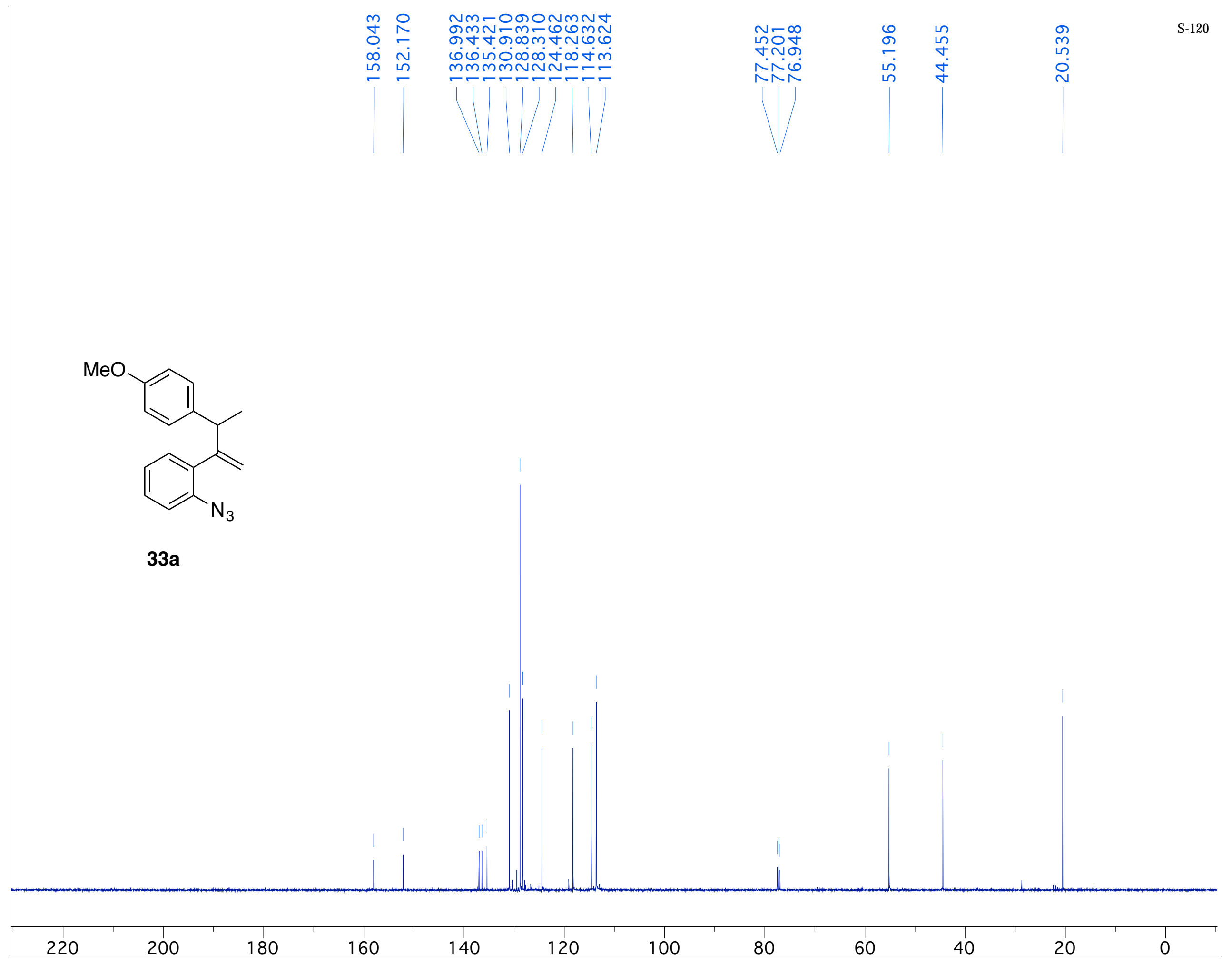




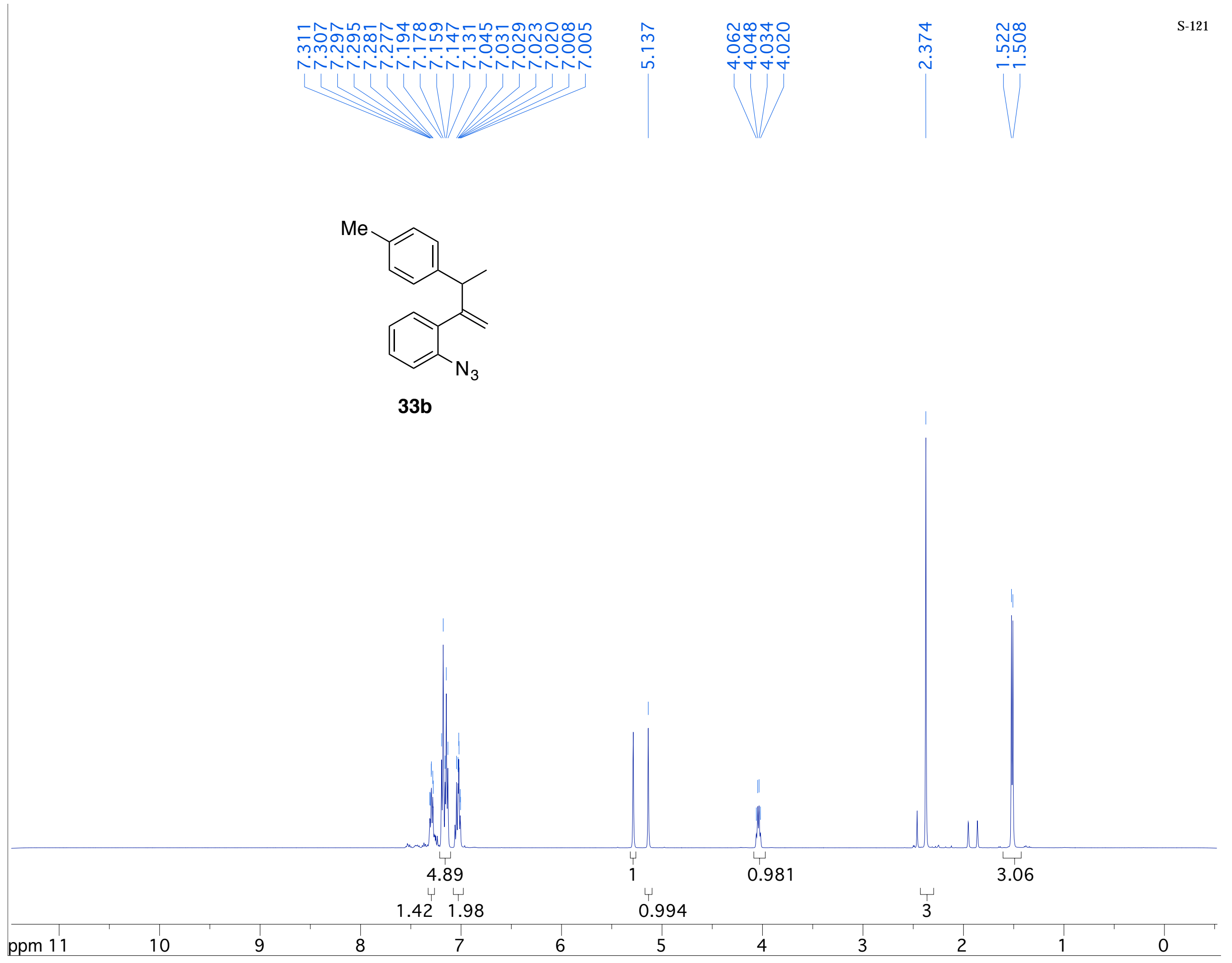




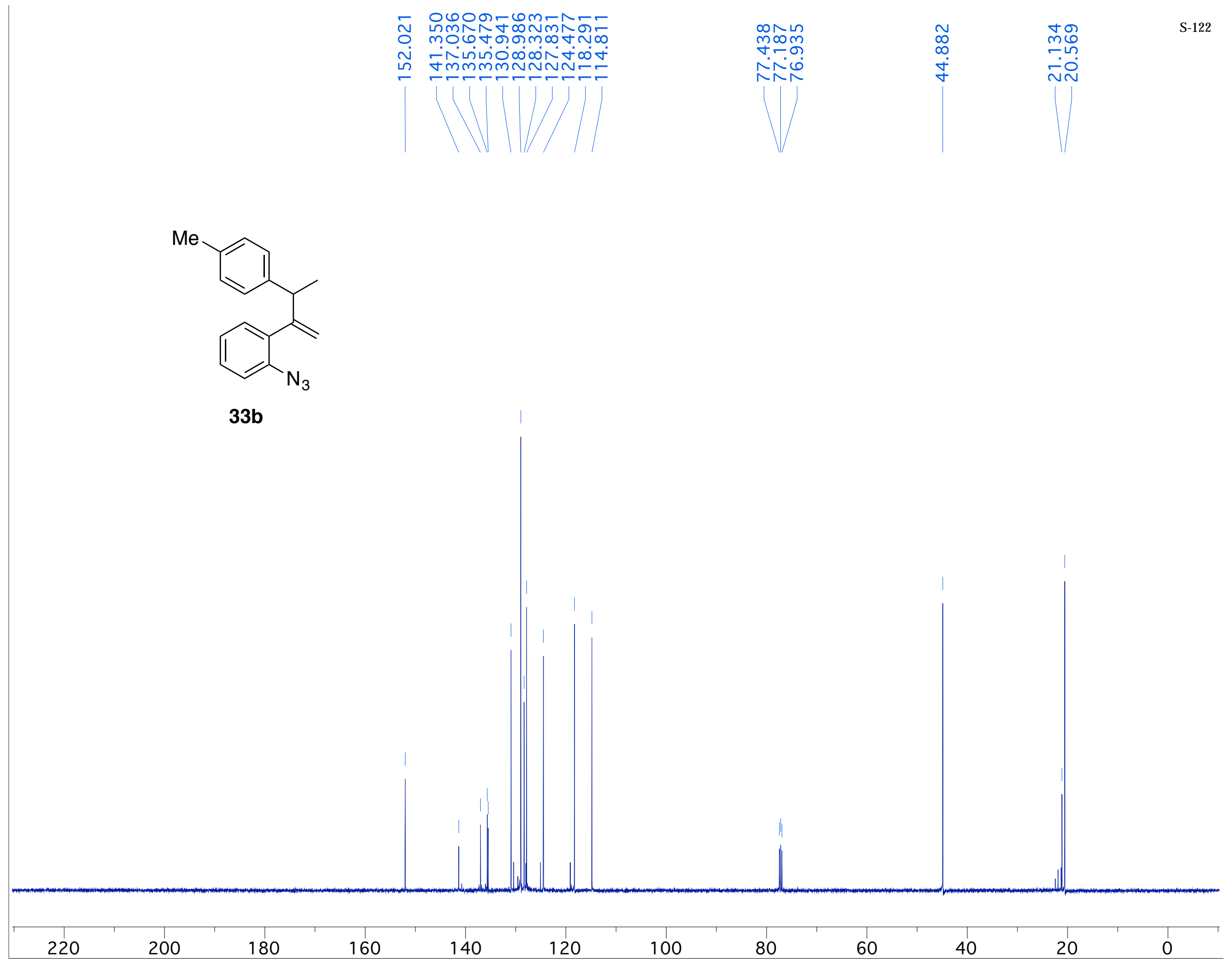




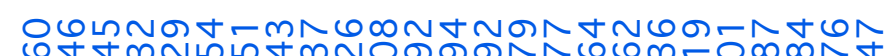

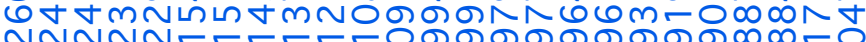

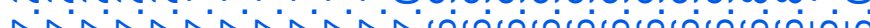<smiles>C=C(c1ccccc1N)C(C)c1ccc(F)cc1</smiles>

33d

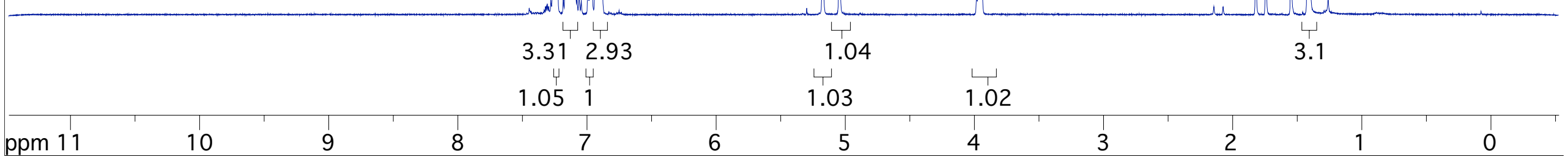




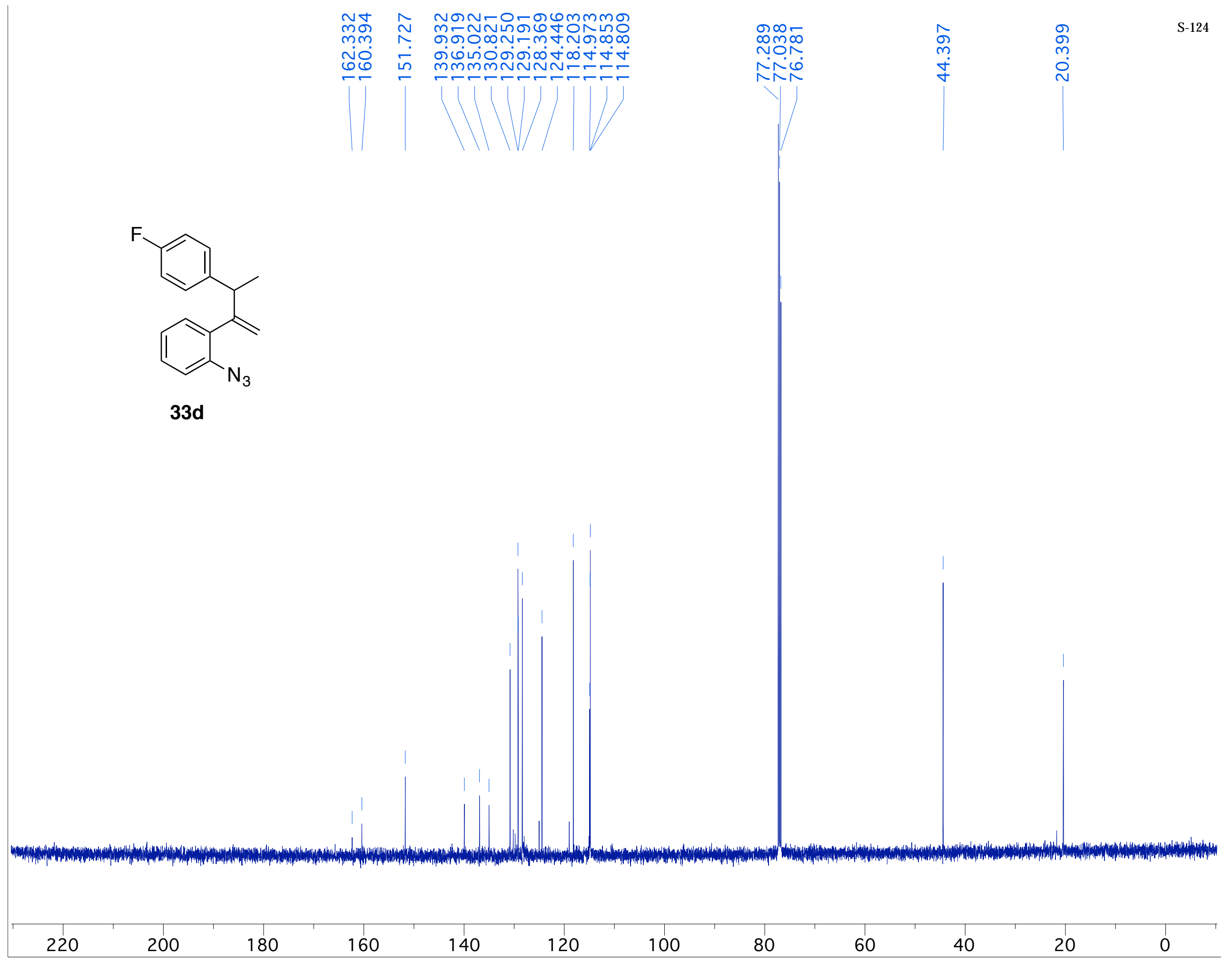




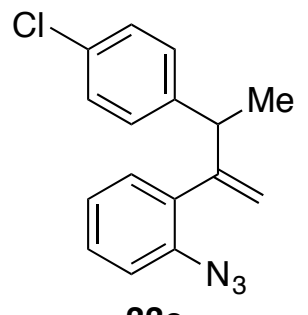

$33 e$

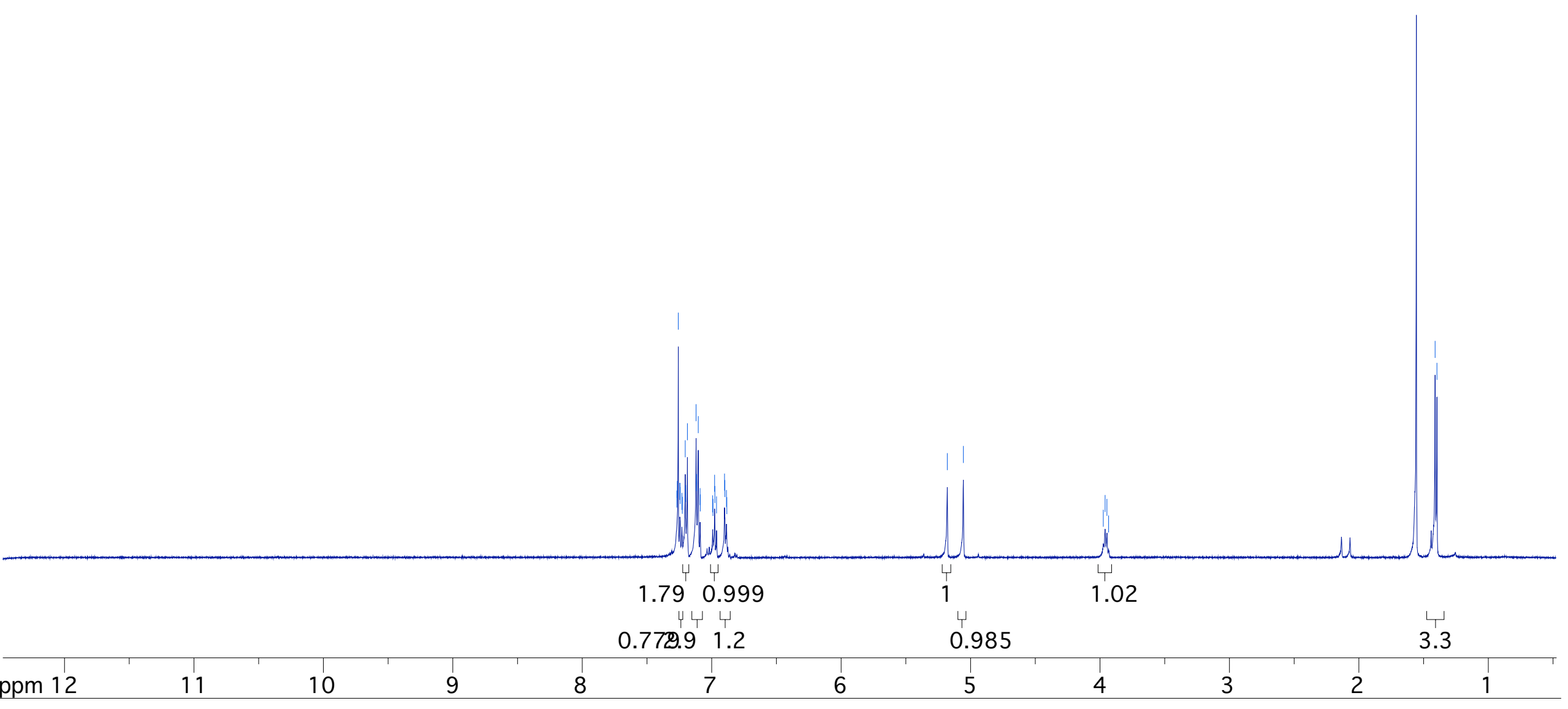




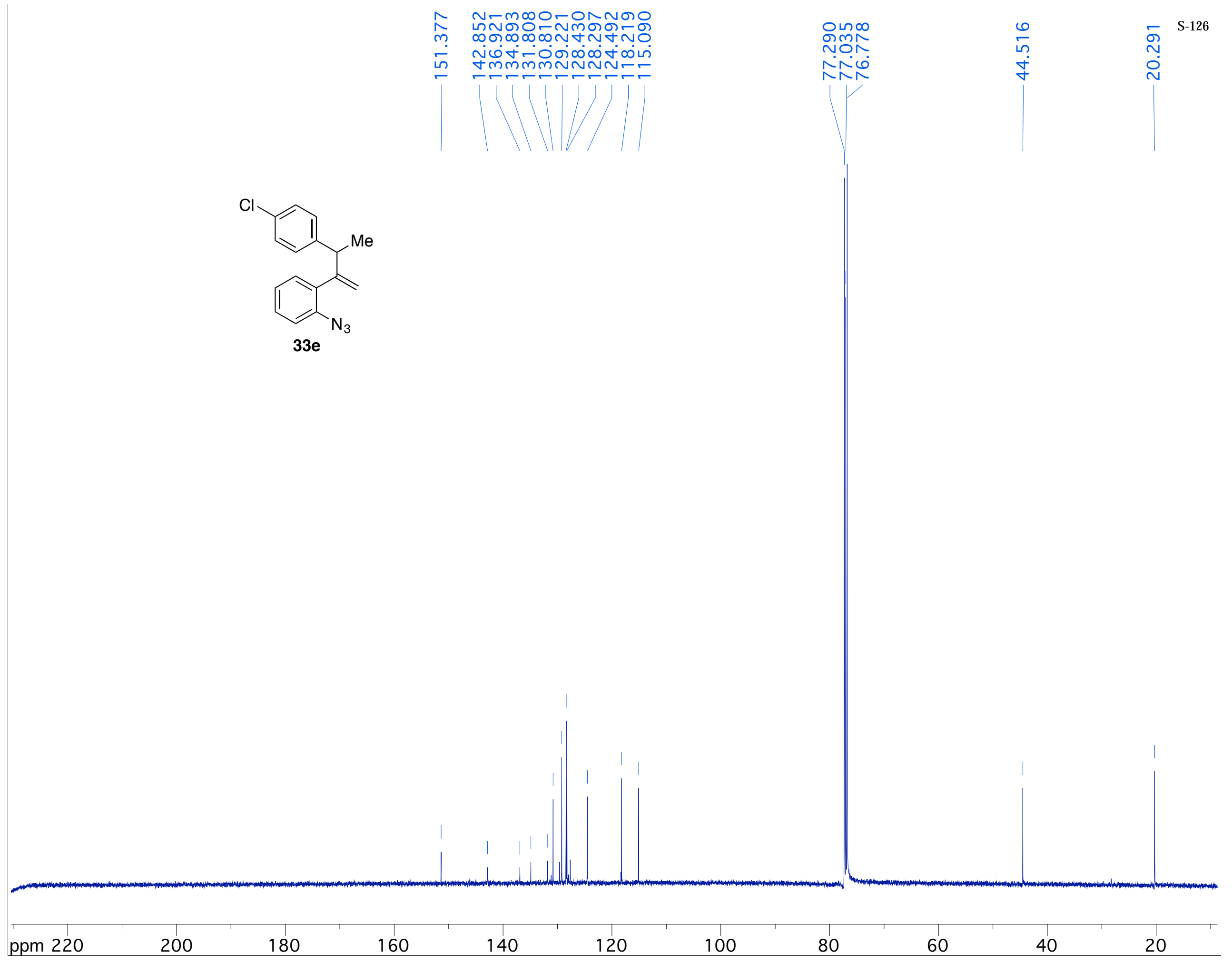


NGด人 N من

+mmm

m.

…ㄴ..

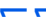

(1)

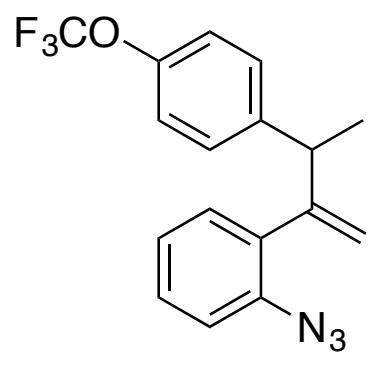

$33 f$ 1

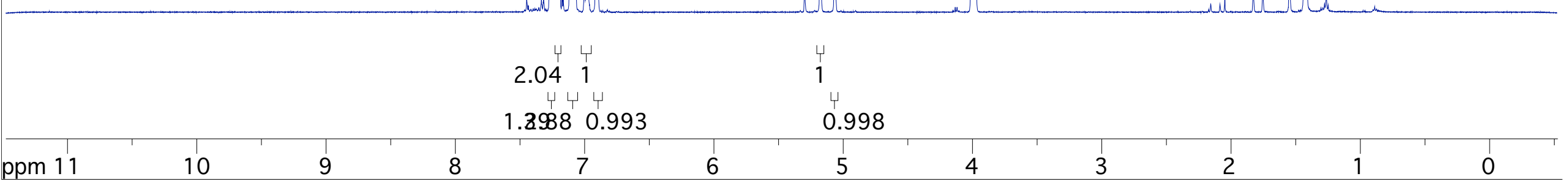




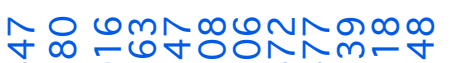

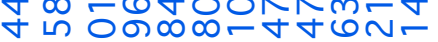

- mं

$\infty \infty 0$

NON

Nं̄ं

ำ

ก $\forall$ mm

$\sim N$



|
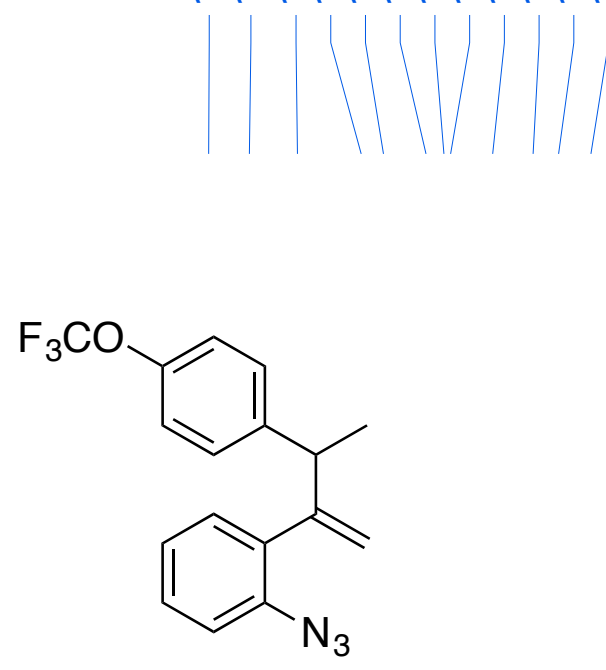

$33 f$

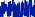

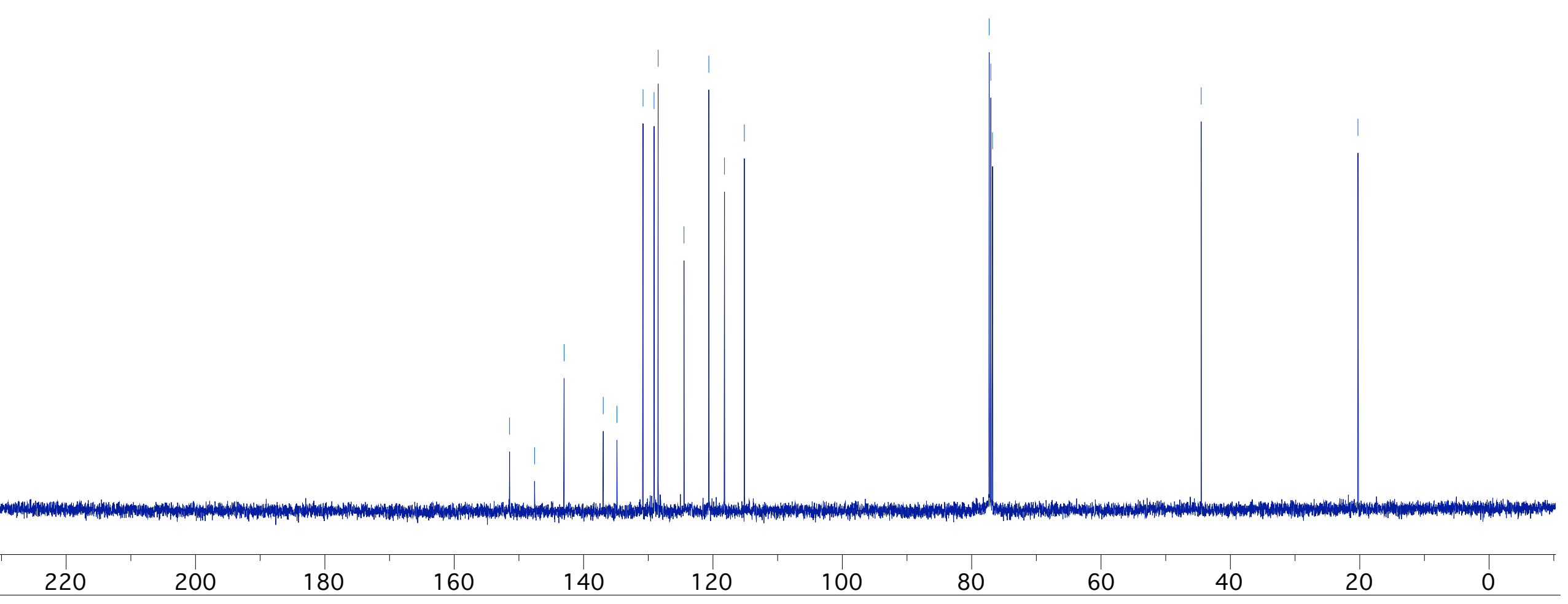




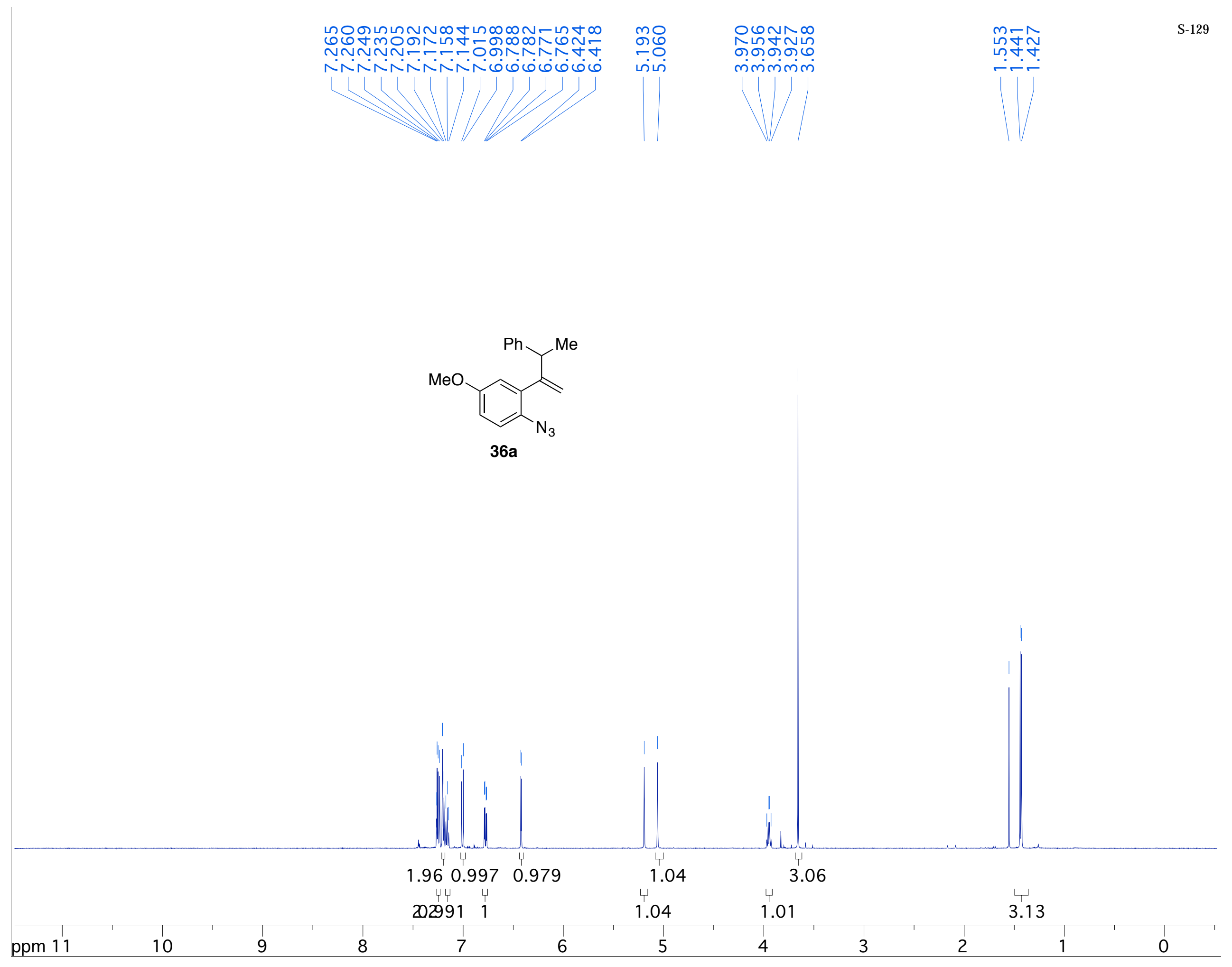




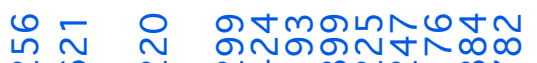

$$
\begin{aligned}
& \text { N } \\
& \text { ம் }
\end{aligned}
$$<smiles>C=C(C(=C)C(c1ccccc1)c1cccc(OC)c1)C(C)C</smiles>

$36 a$

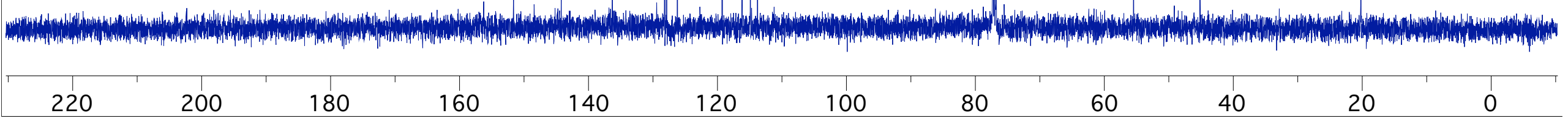




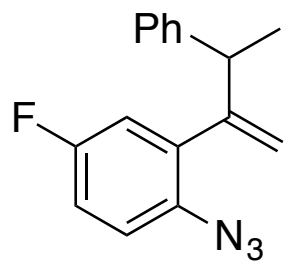

36d
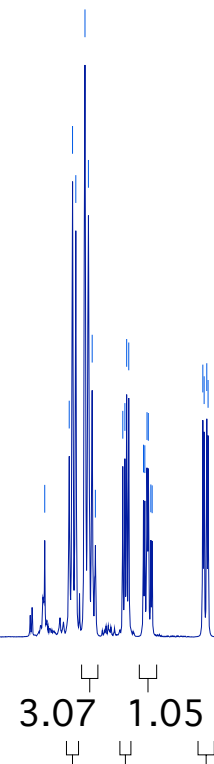

2.071 .031

$\frac{1}{1.05}$




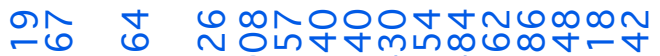

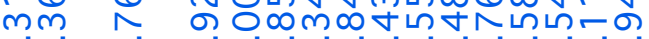

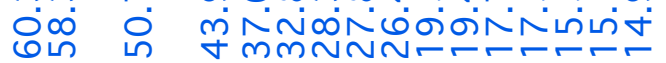

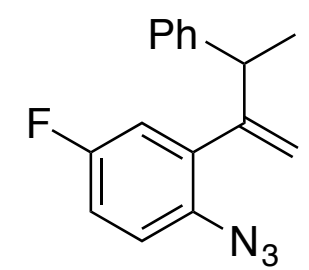

36d

ppm 220




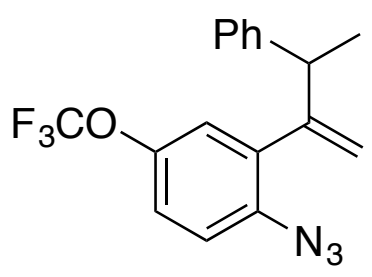

$36 f$

\subsection{5}

1.07 


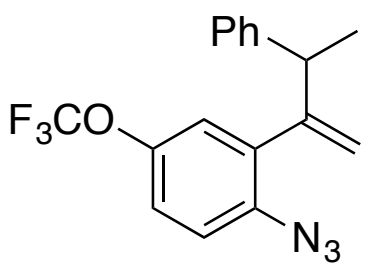

$36 f$ 


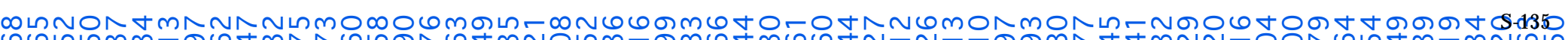
6

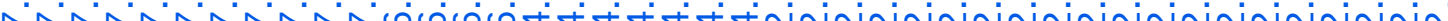
LLLLLLLLUL

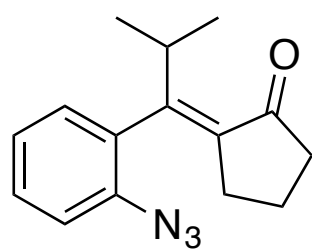

12

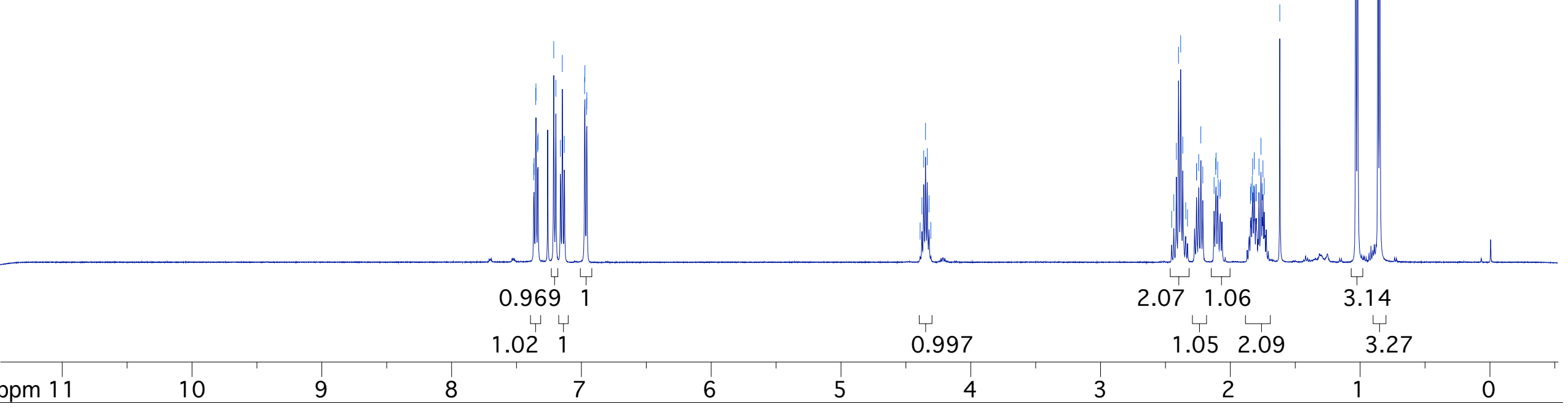




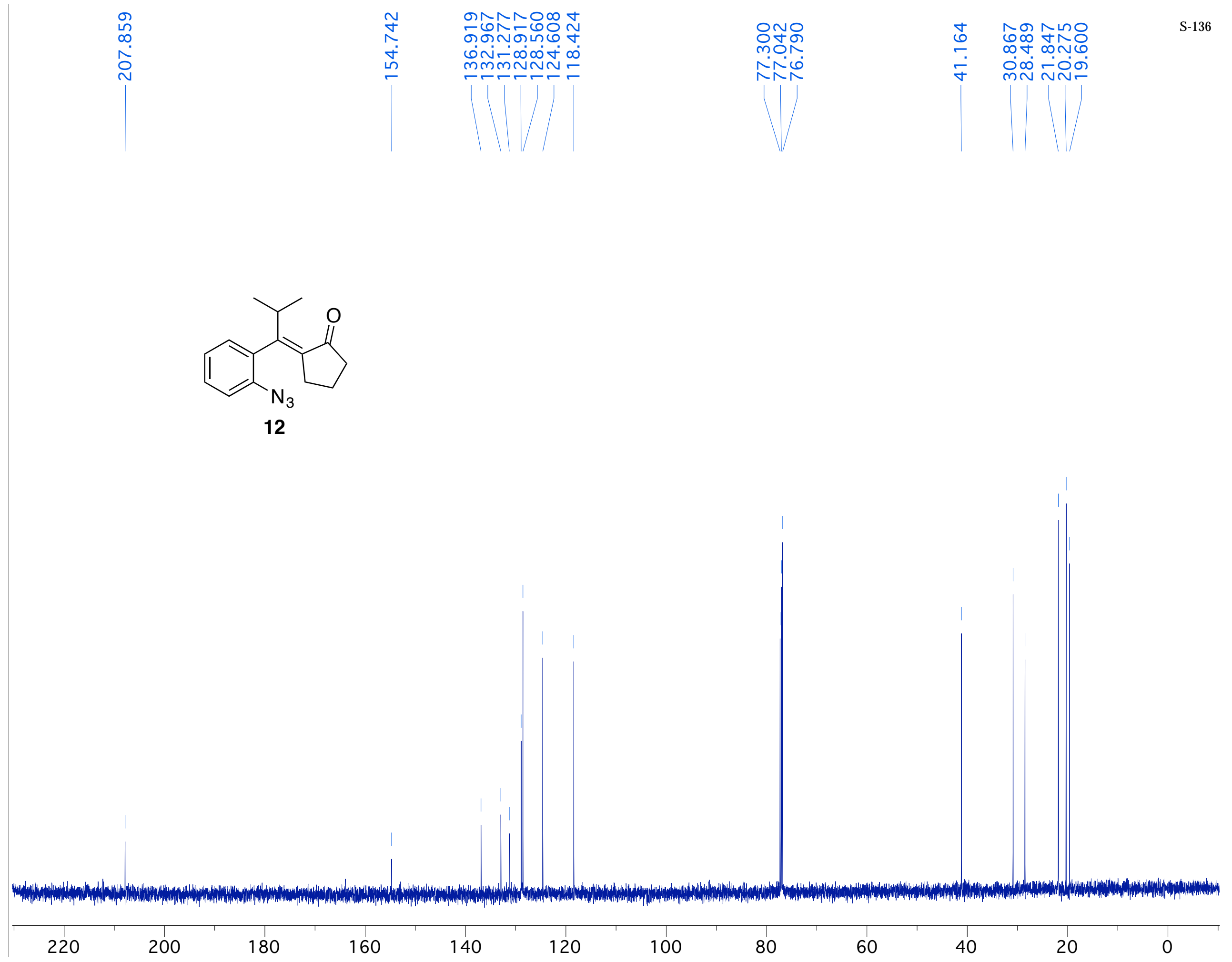




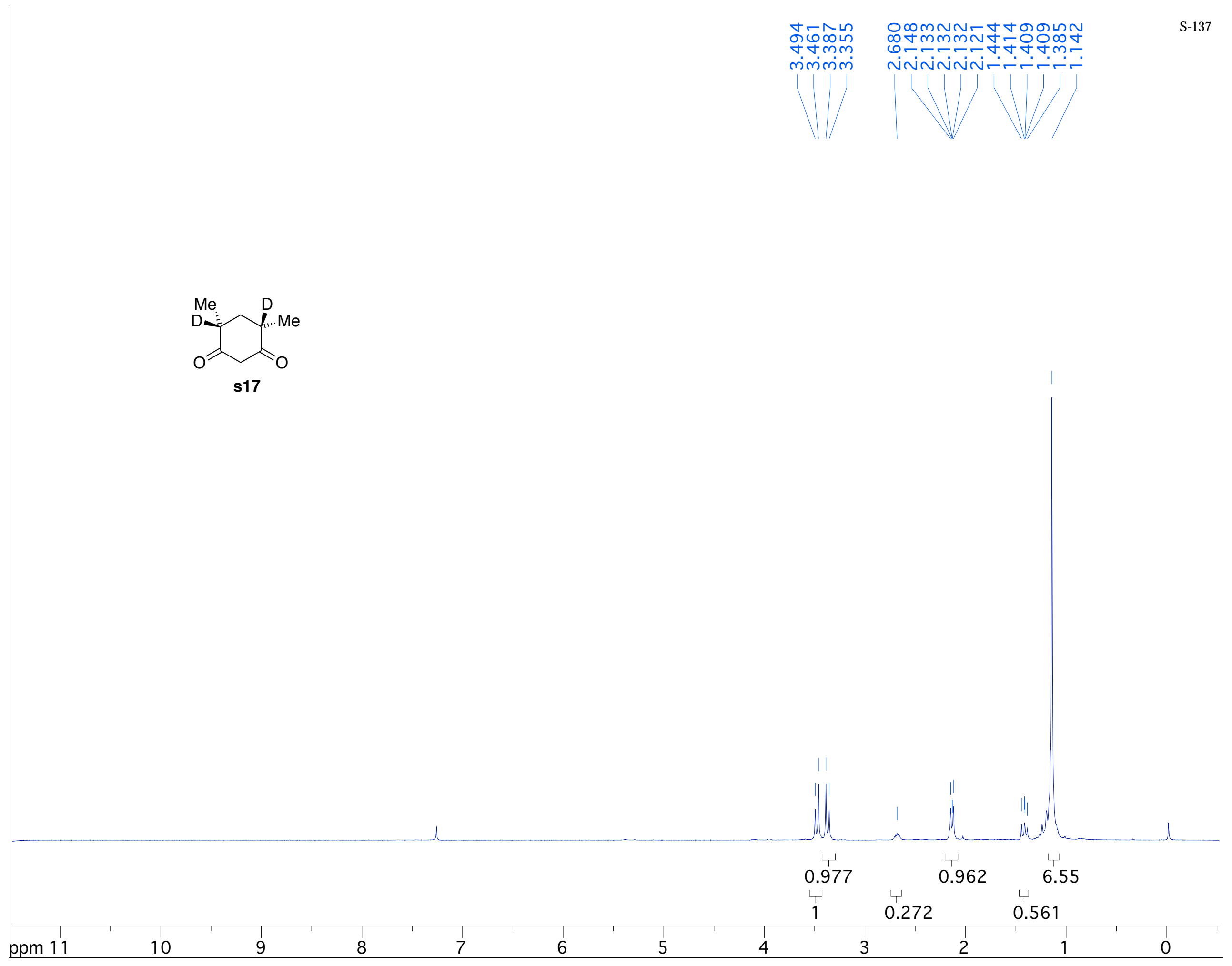




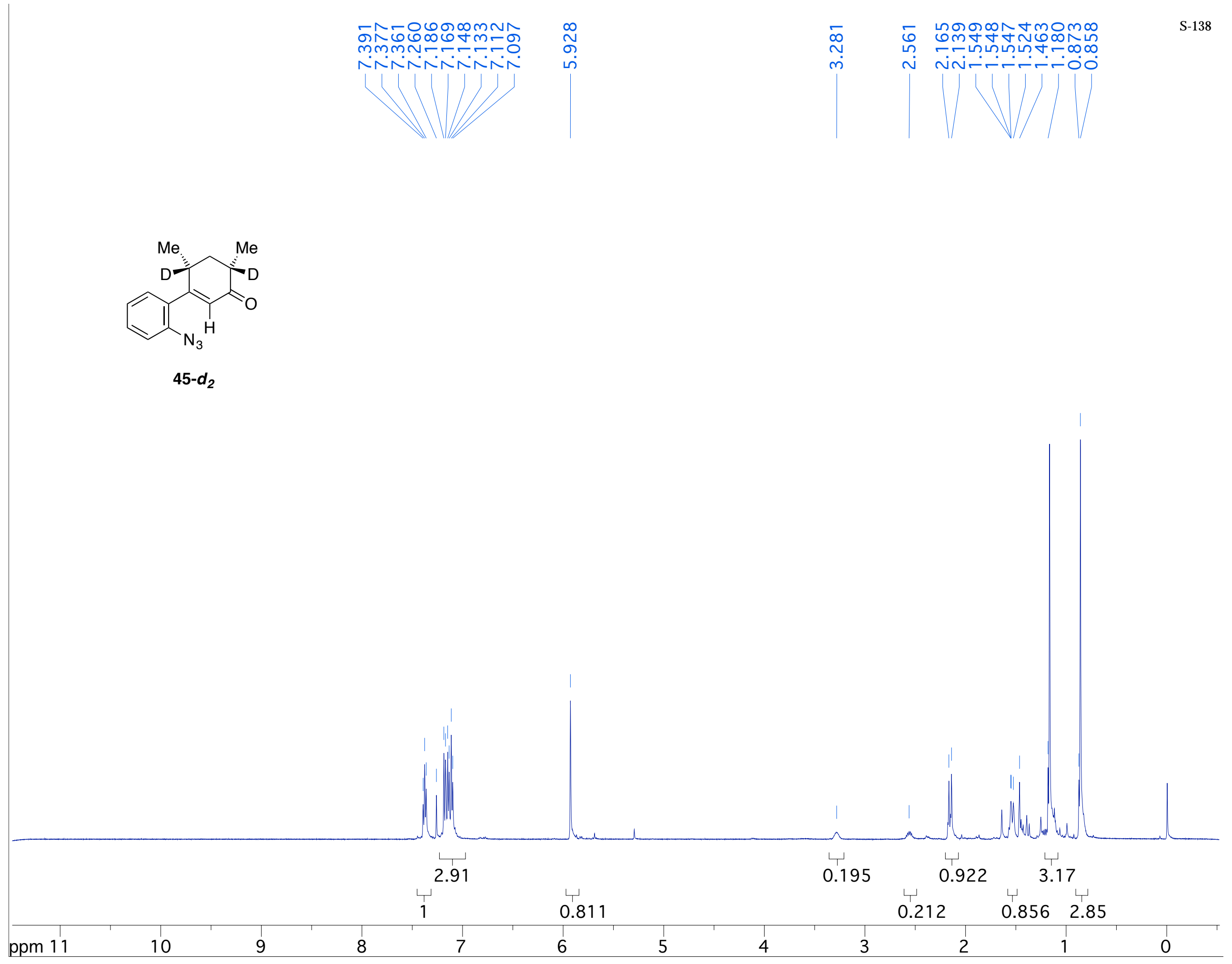




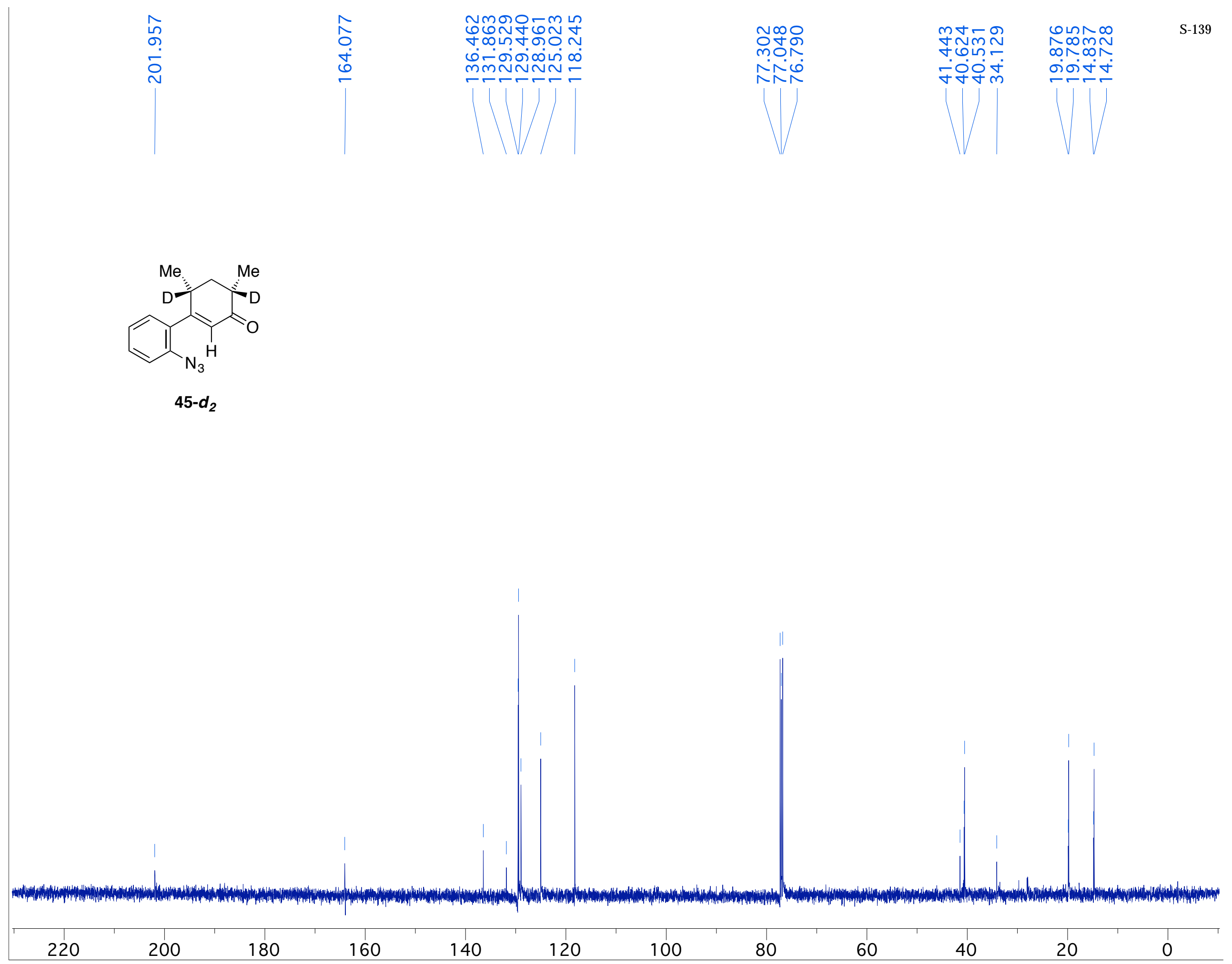




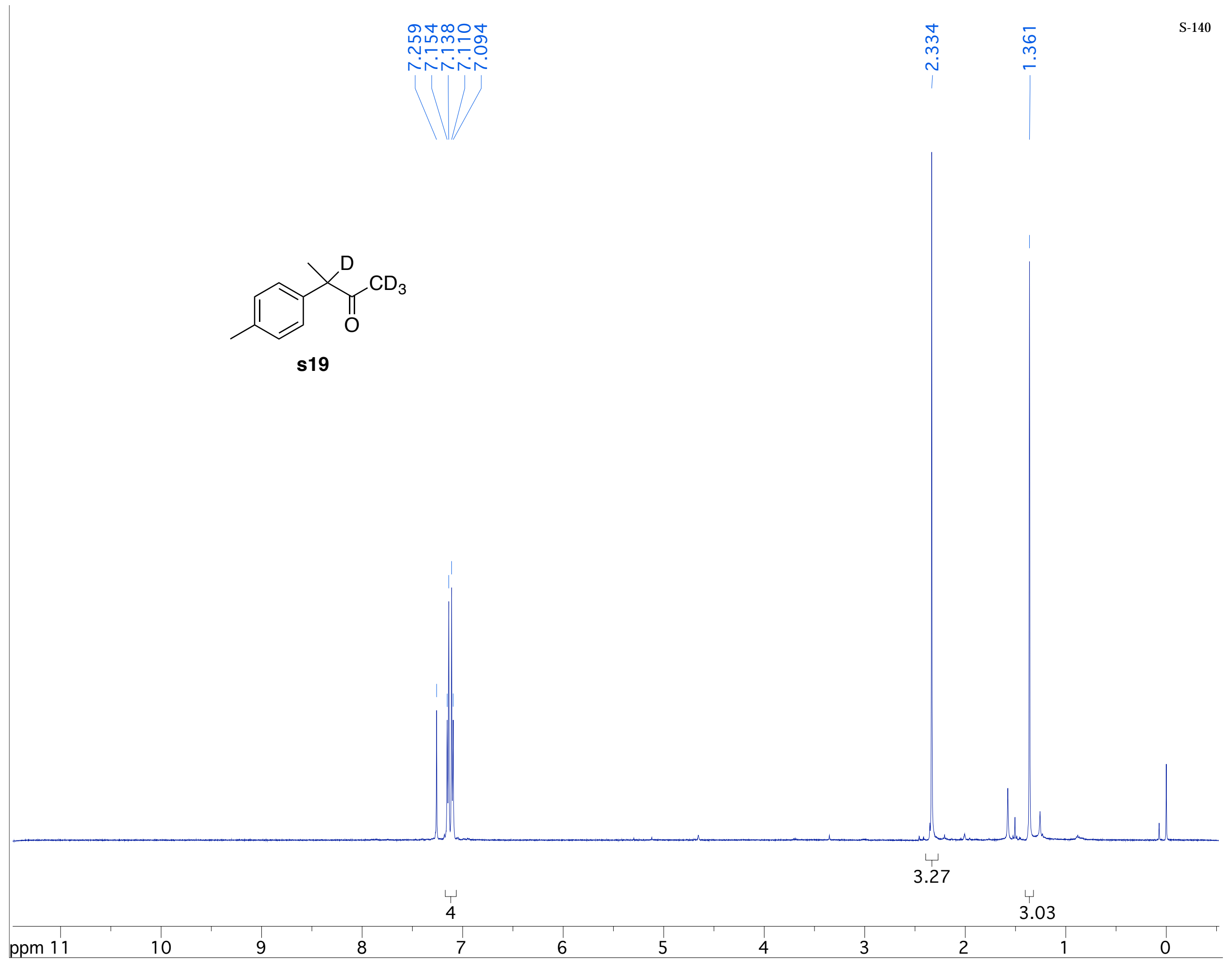




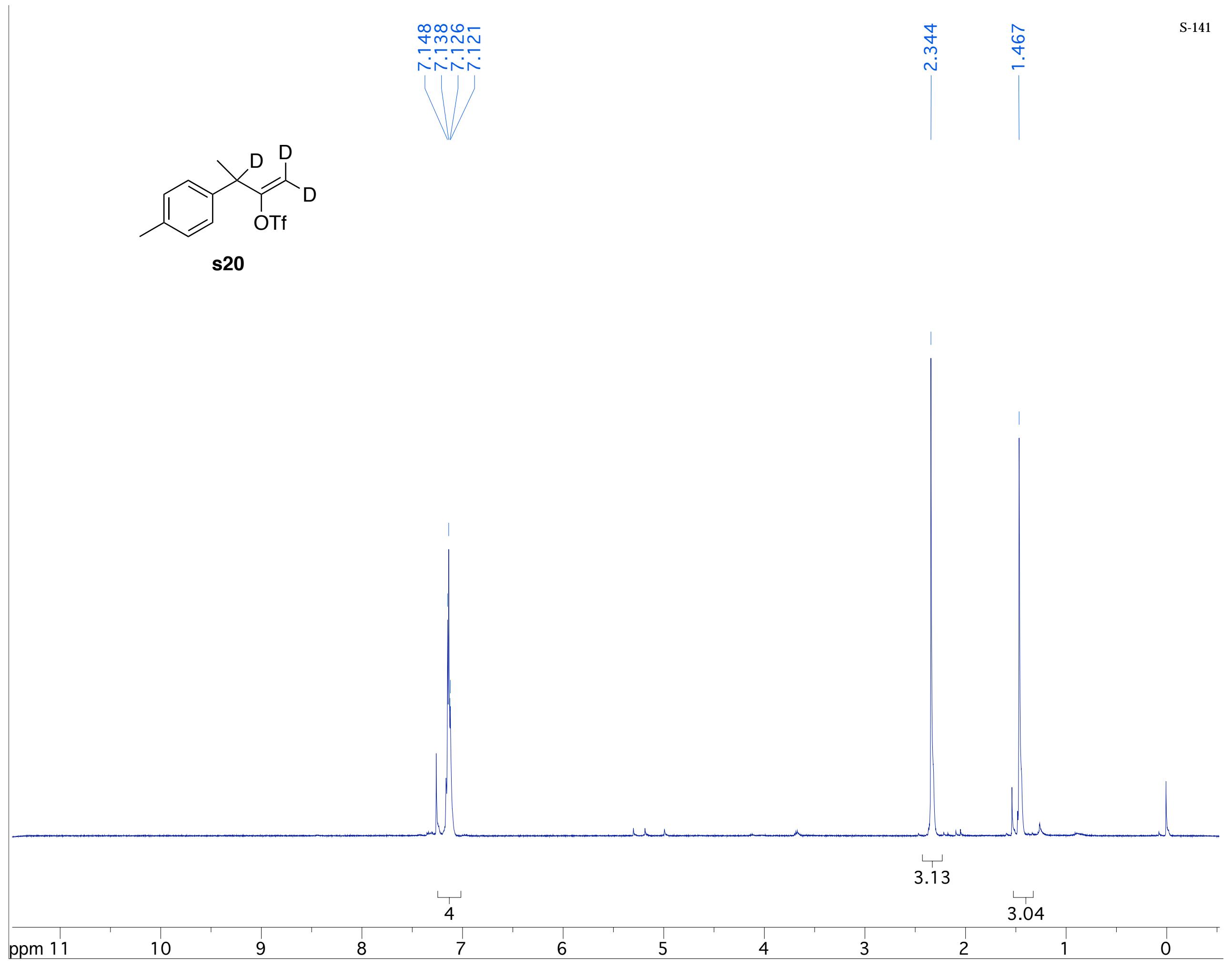




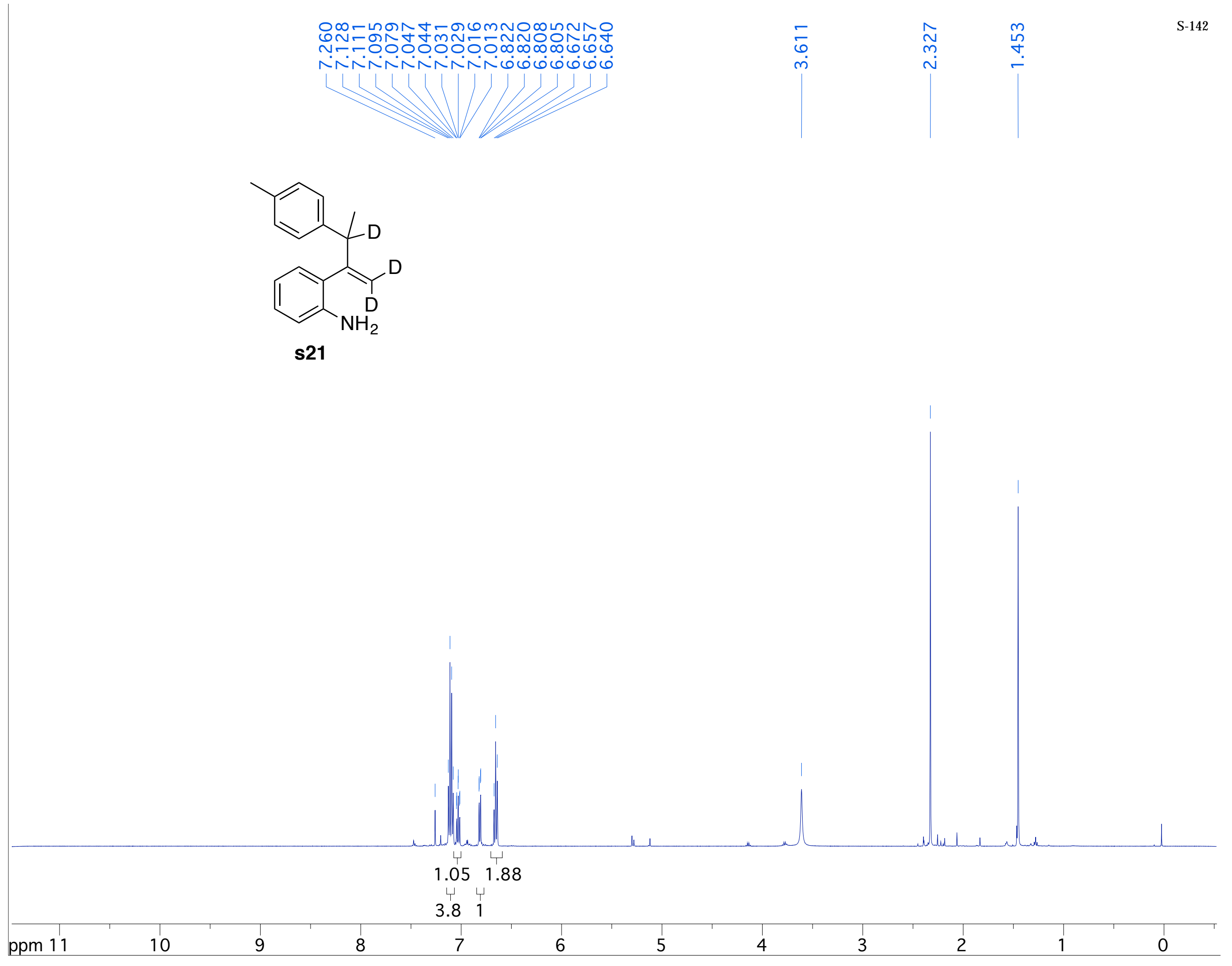




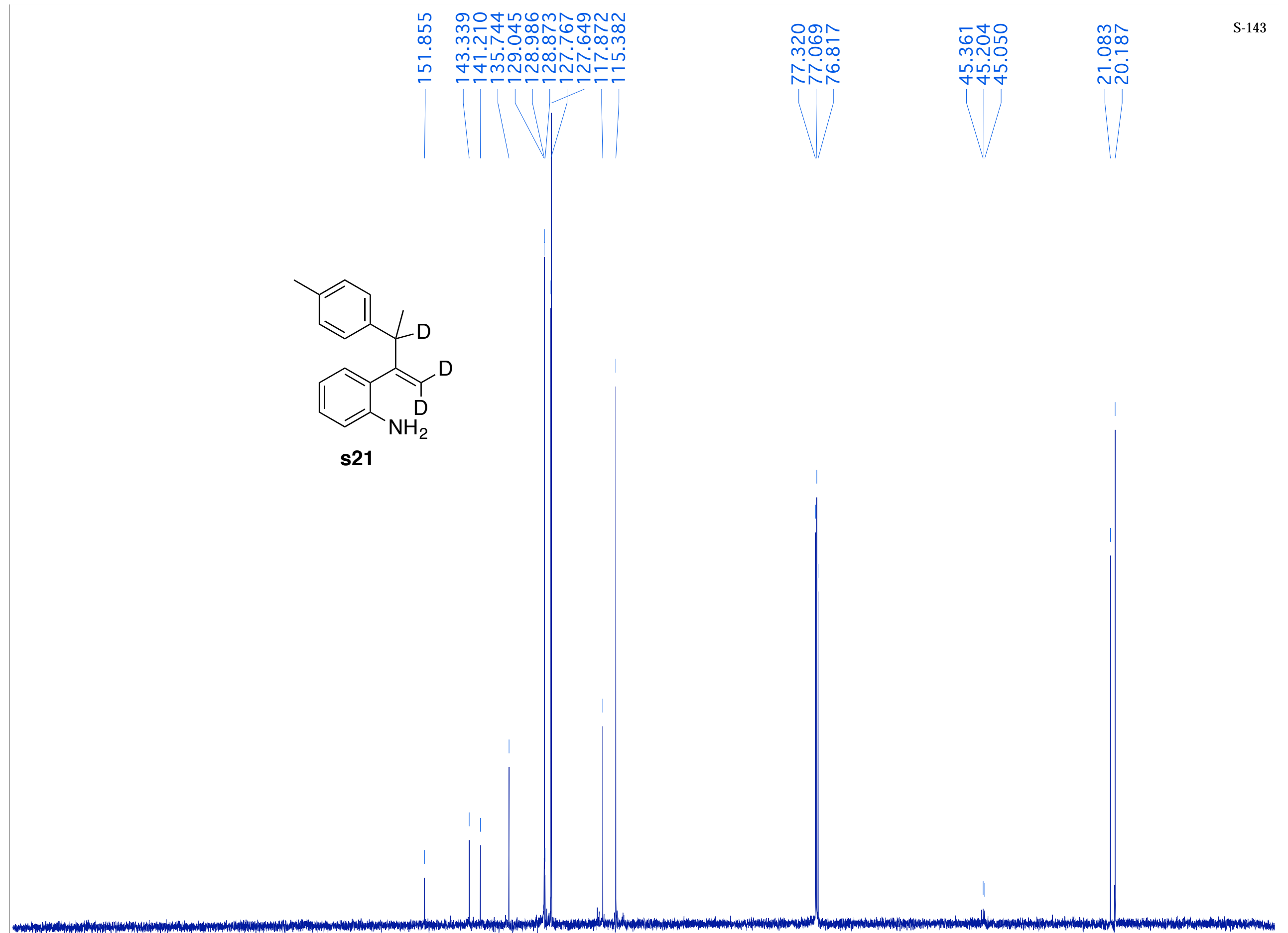

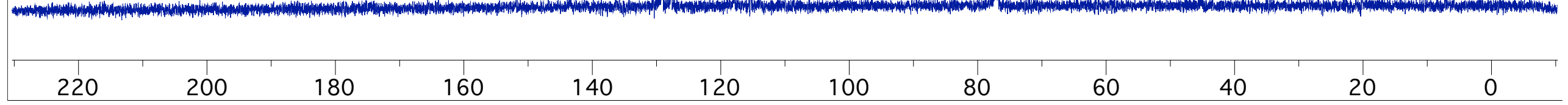


NNNNNNNN6060606

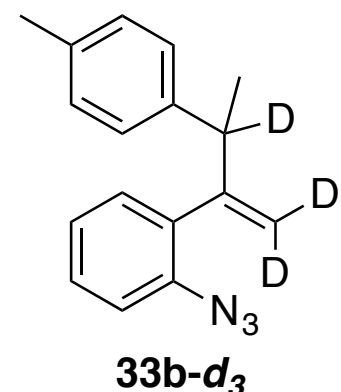

$3 b-d_{3}$

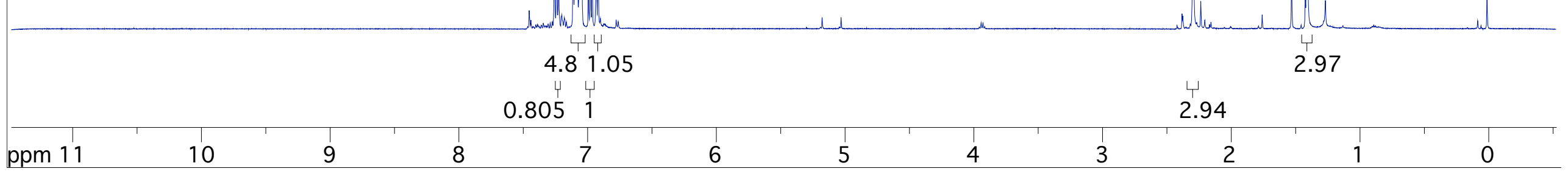




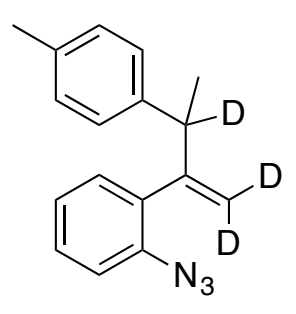

$33 \mathrm{~b}-d_{3}$

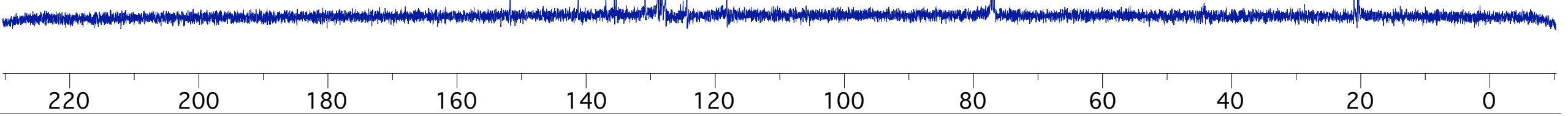



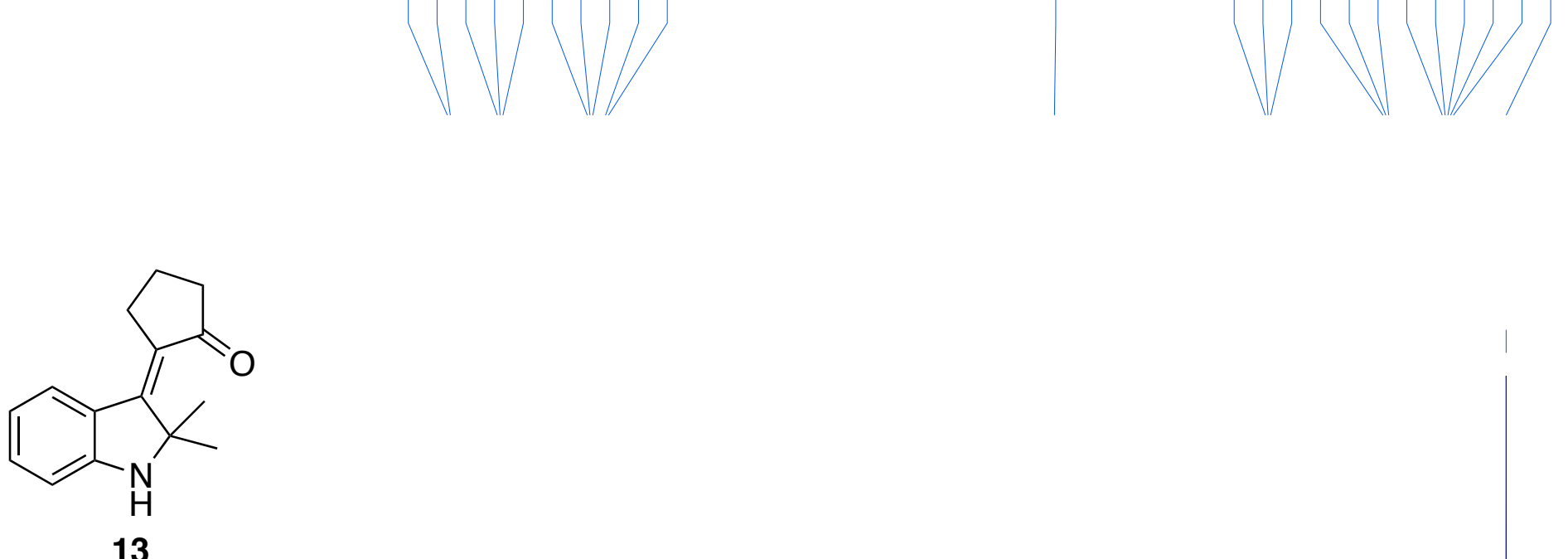

13

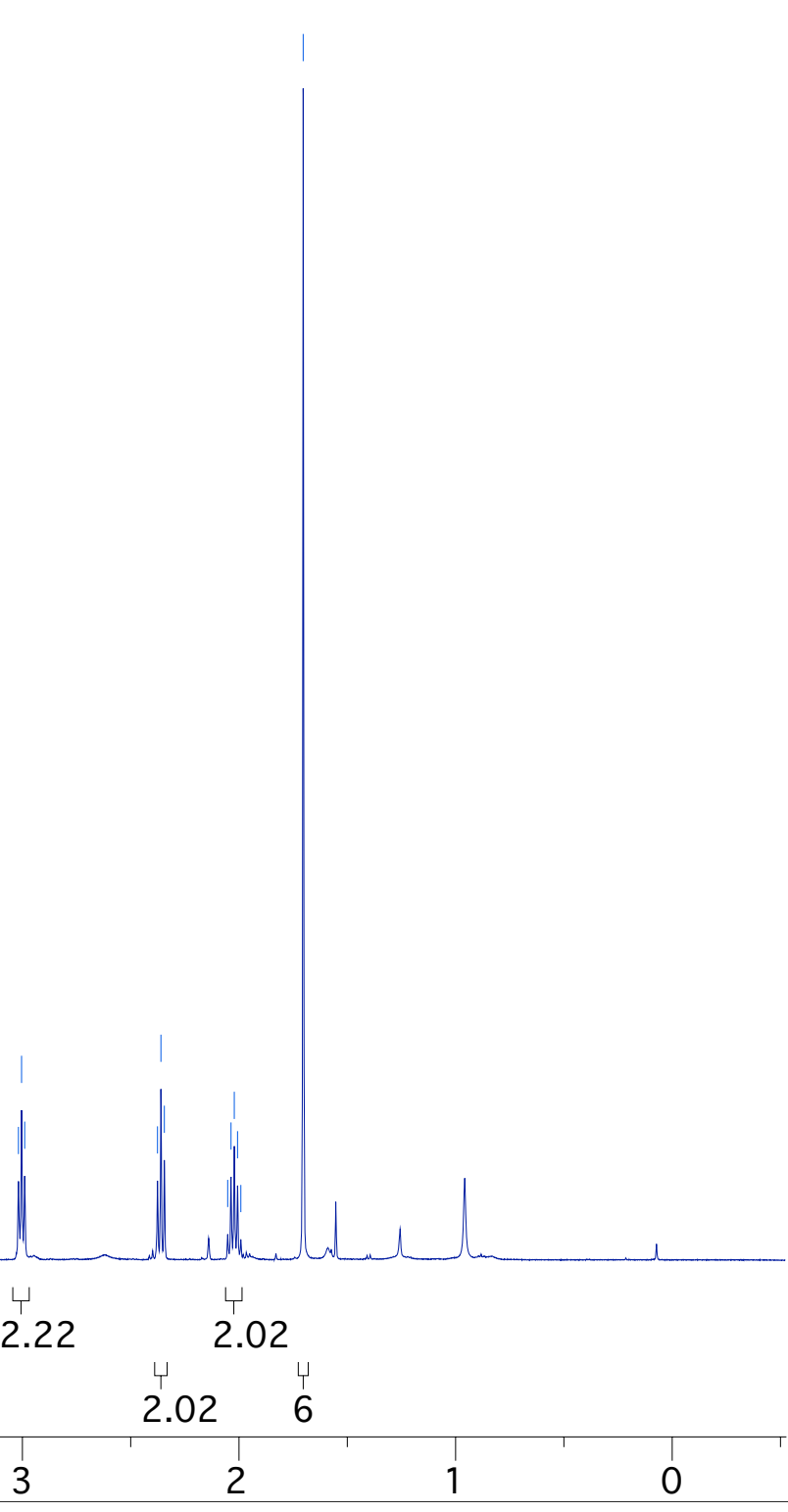




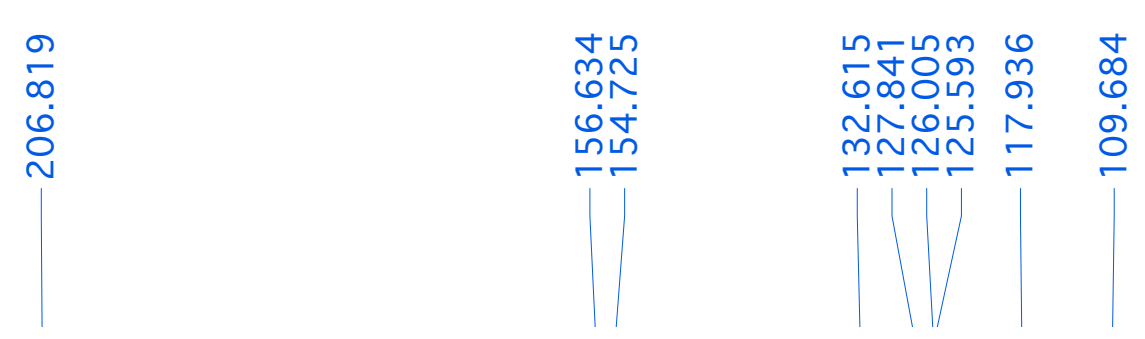<smiles>CC1(C)Nc2ccccc2C1=C1CCCC1=O</smiles>

13

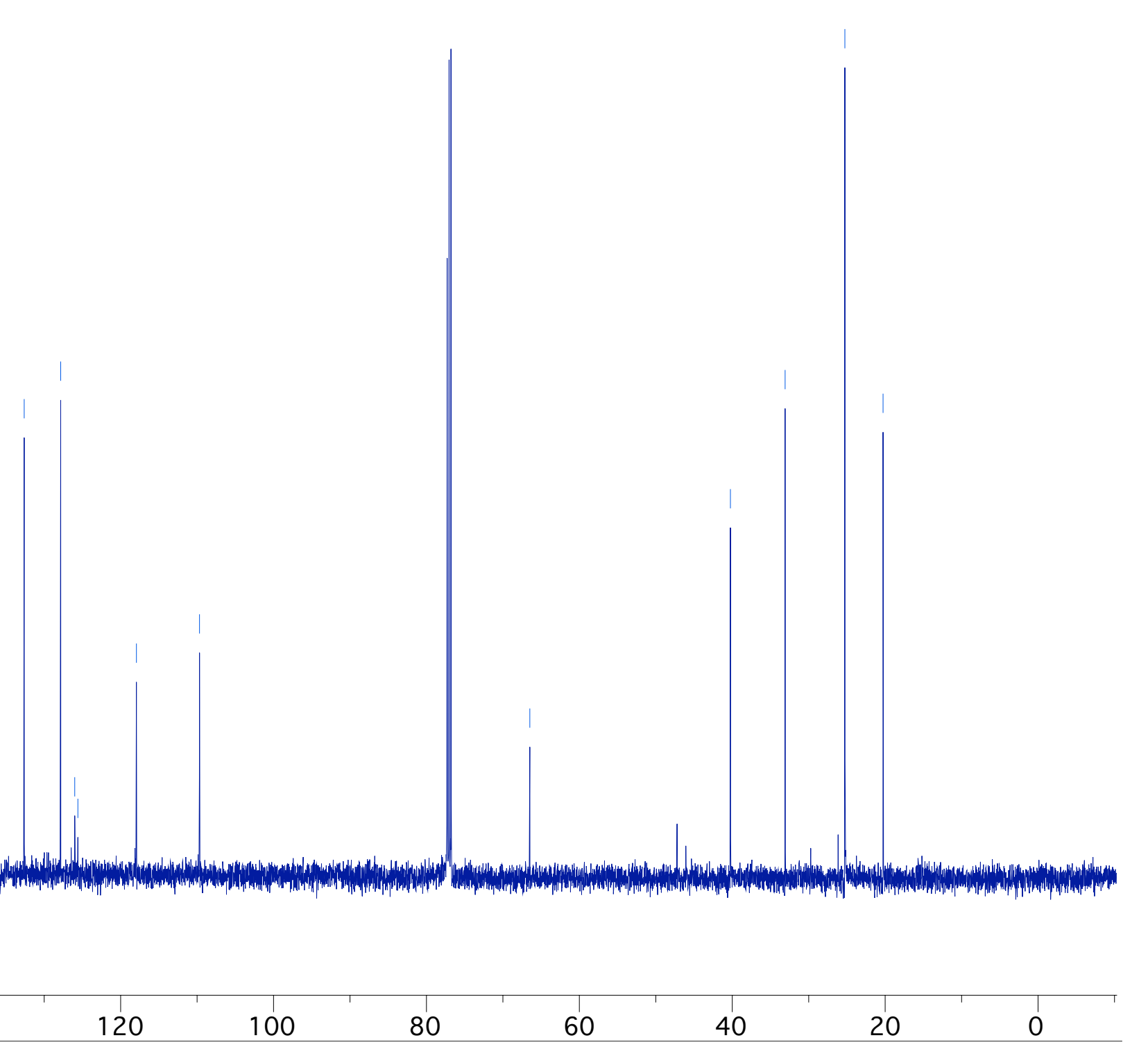




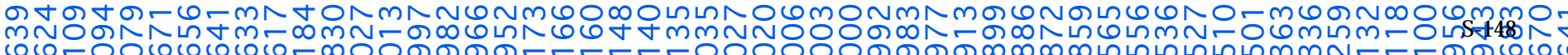

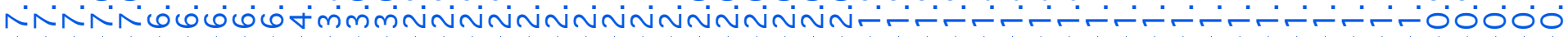

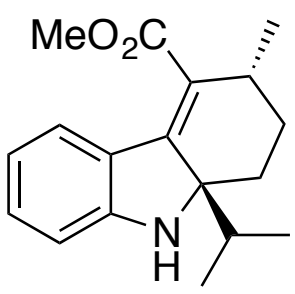

19

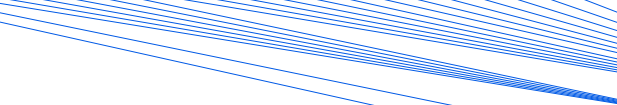




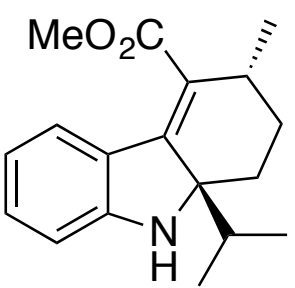

19 


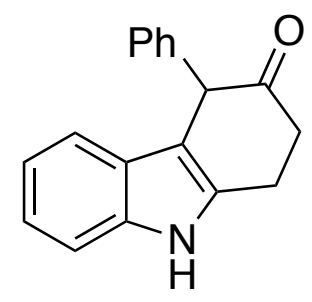

$26 a$

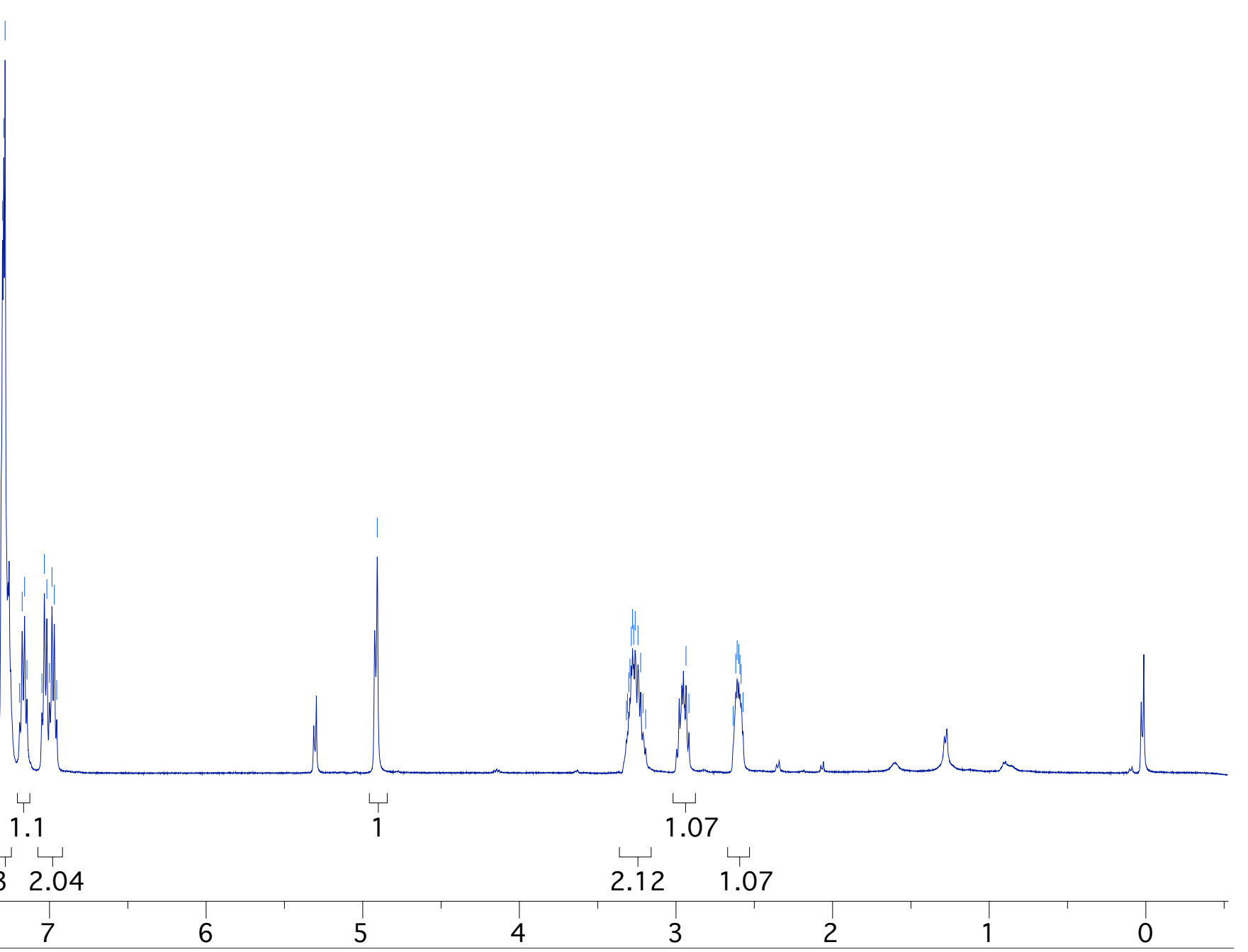


<smiles>O=C1CCc2[nH]c3ccccc3c2C1c1ccccc1</smiles>

26a

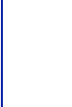


<smiles>O=C1CCc2[nH]c3ccccc3c2C1CO</smiles>

26b<smiles>CC1=C2c3ccccc3NC2CCC1=O</smiles>

26b'

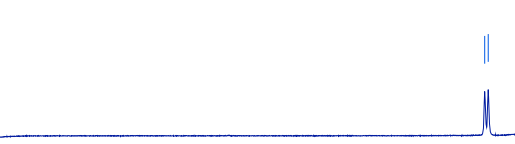

$\begin{array}{lll}1.02 & 0.40208 & 0.214\end{array}$

$\begin{array}{lllll}0.204 & 1 & 1 & 1.22\end{array}$
0.175

5 illuh

$$
\begin{array}{lll}
0.976 & 1.05 & 1.13 \\
& & \\
2.07 & 0.593
\end{array}
$$

3
3.15 
<smiles>COC(=O)C1=C2c3ccccc3NC2CN1C(=O)OCc1ccccc1</smiles>

26d

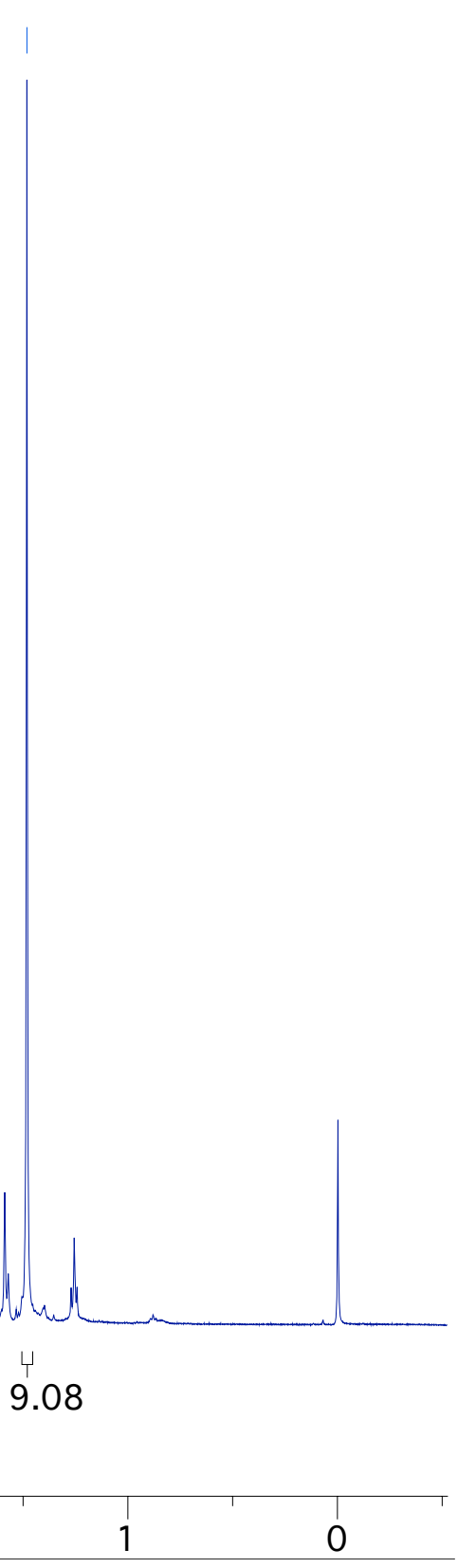

8

6




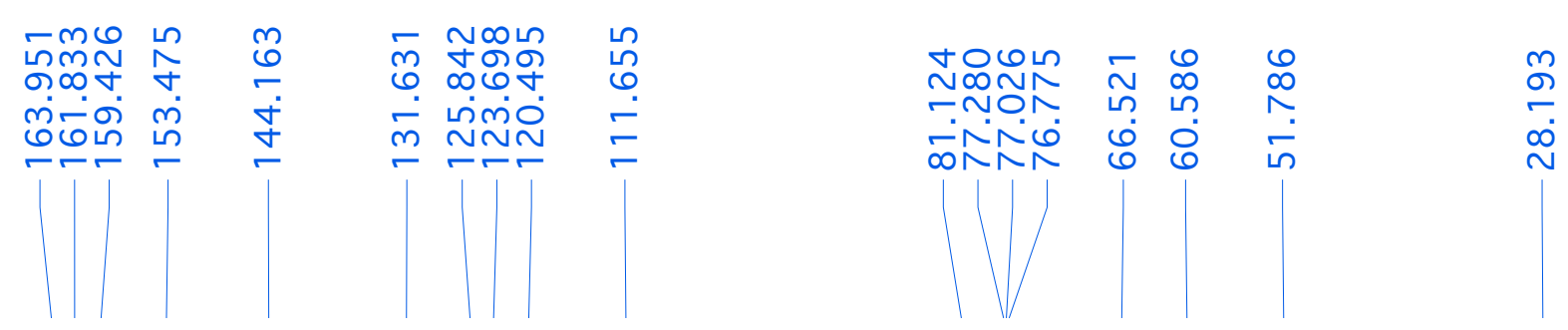

ตั<smiles>COC(=O)C1=C2c3ccccc3NC2CN1C(=O)OC(C)(C)C</smiles>

26d

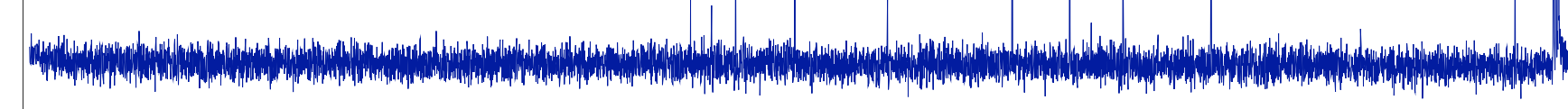


$6 \pi 0+\infty a+\infty-6 N$ onvining NinNNNNNN<smiles>O=C(C1CCCCC1)C1CCCc2[nH]c3ccccc3c21</smiles>

$26 f$
mom-G-

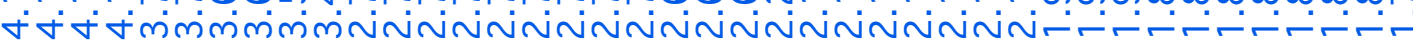

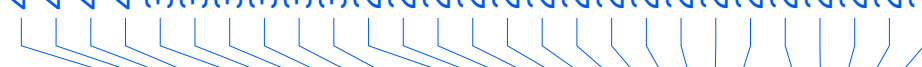


<smiles>O=C(C1CCCc2[nH]c3ccccc3c21)N1CCOCC1</smiles>

$26 f$ 


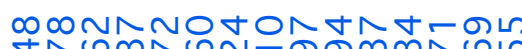
(0)

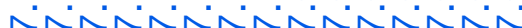

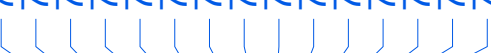

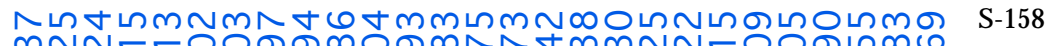
舟 mกNiNiN<smiles>CC1CCCc2[nH]c3ccccc3c21</smiles>

28

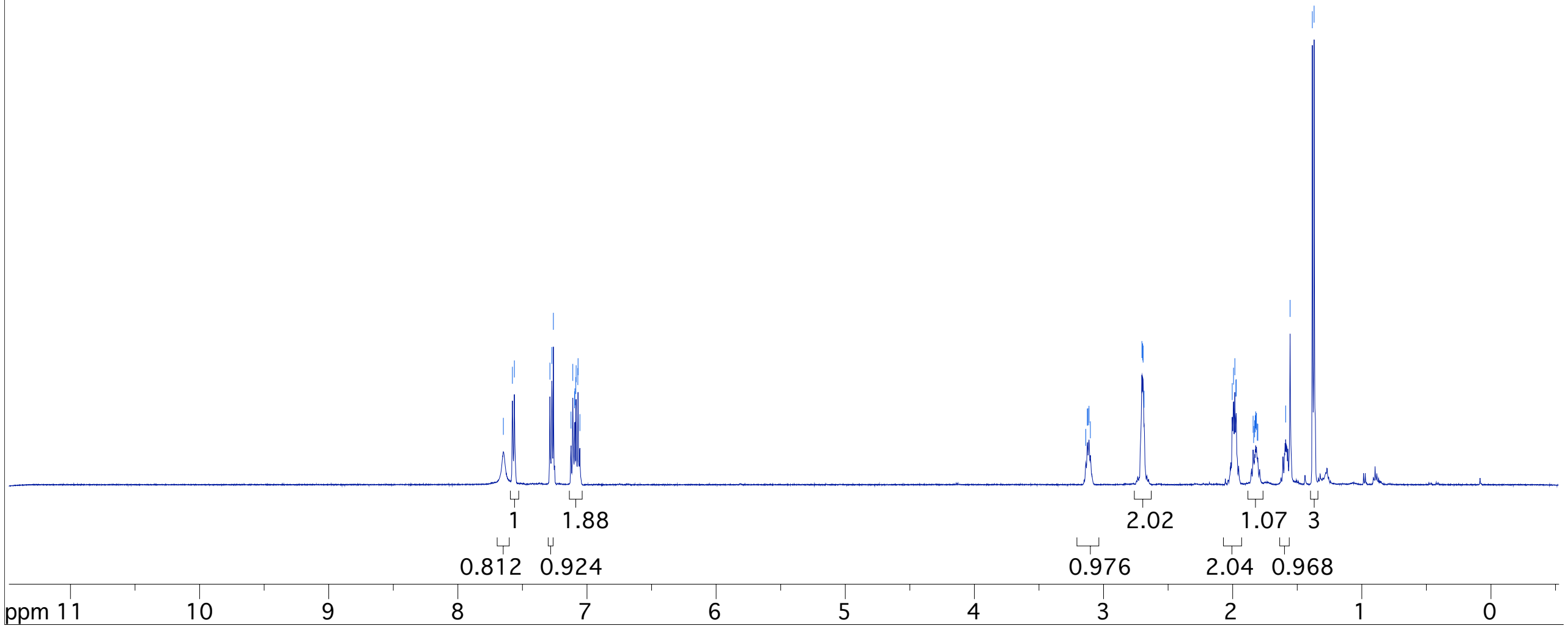




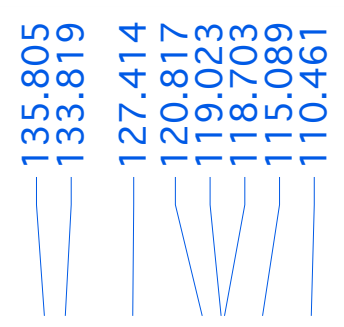

ำ์

- กோட

m m

N Ní-

NNG

$m \sim N \sim N$

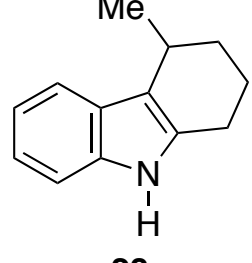

28

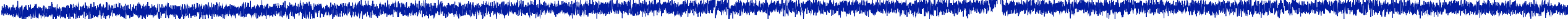




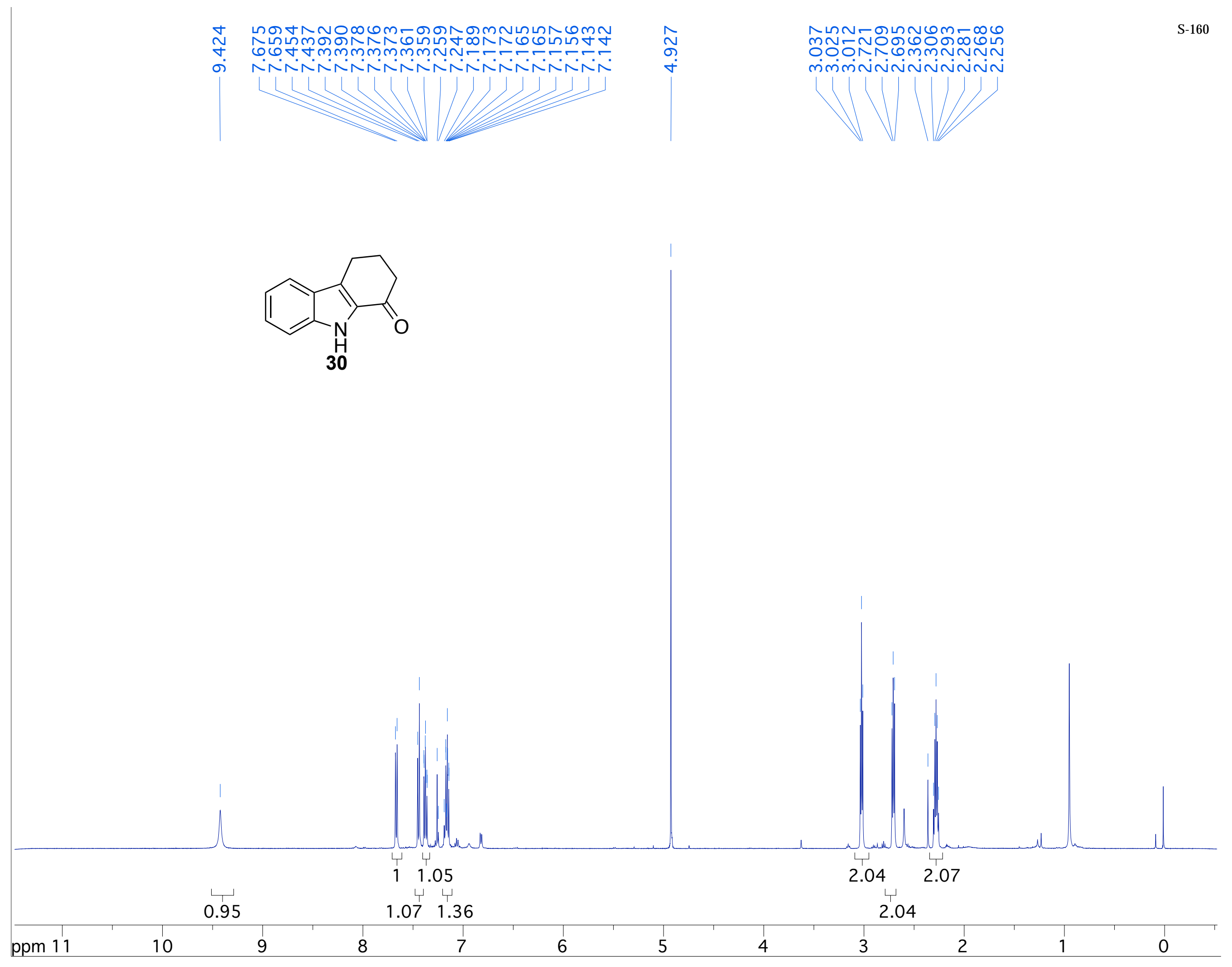




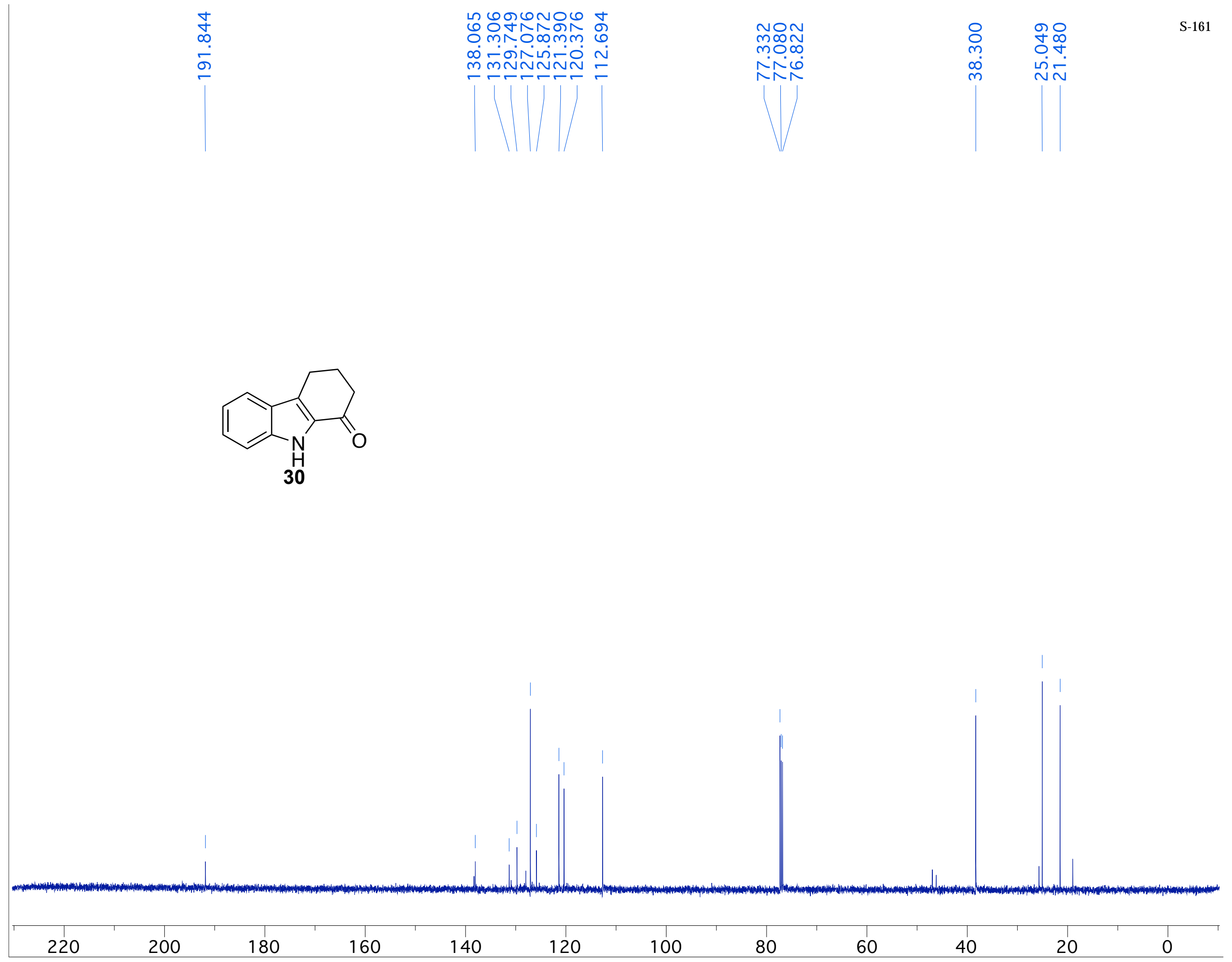




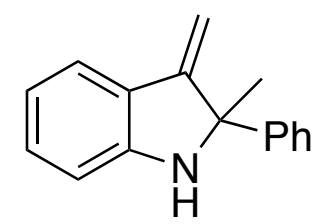

$38 \mathrm{c}$

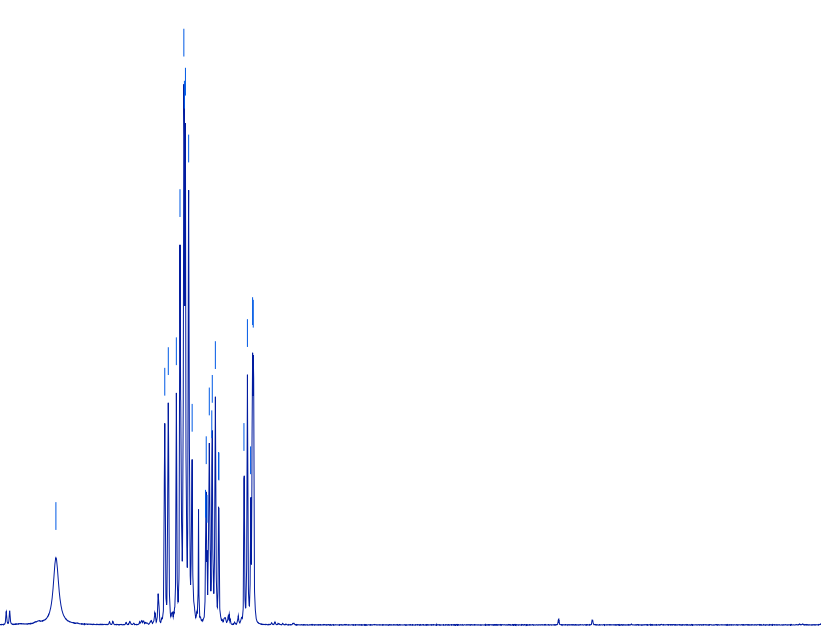

1.0420 .968

$0.898 \quad 5.02 \quad 1.02$

$8 \quad 7$

1.01 


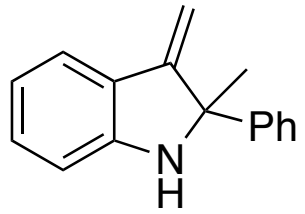

$38 c$

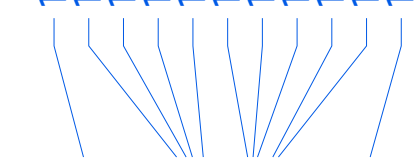




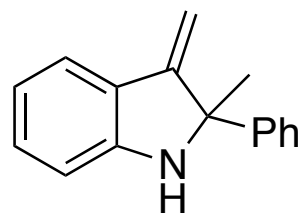

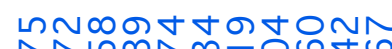
N

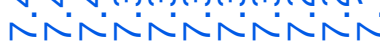

mon-

N

LUUHJ

ต่ต்

$37 c$
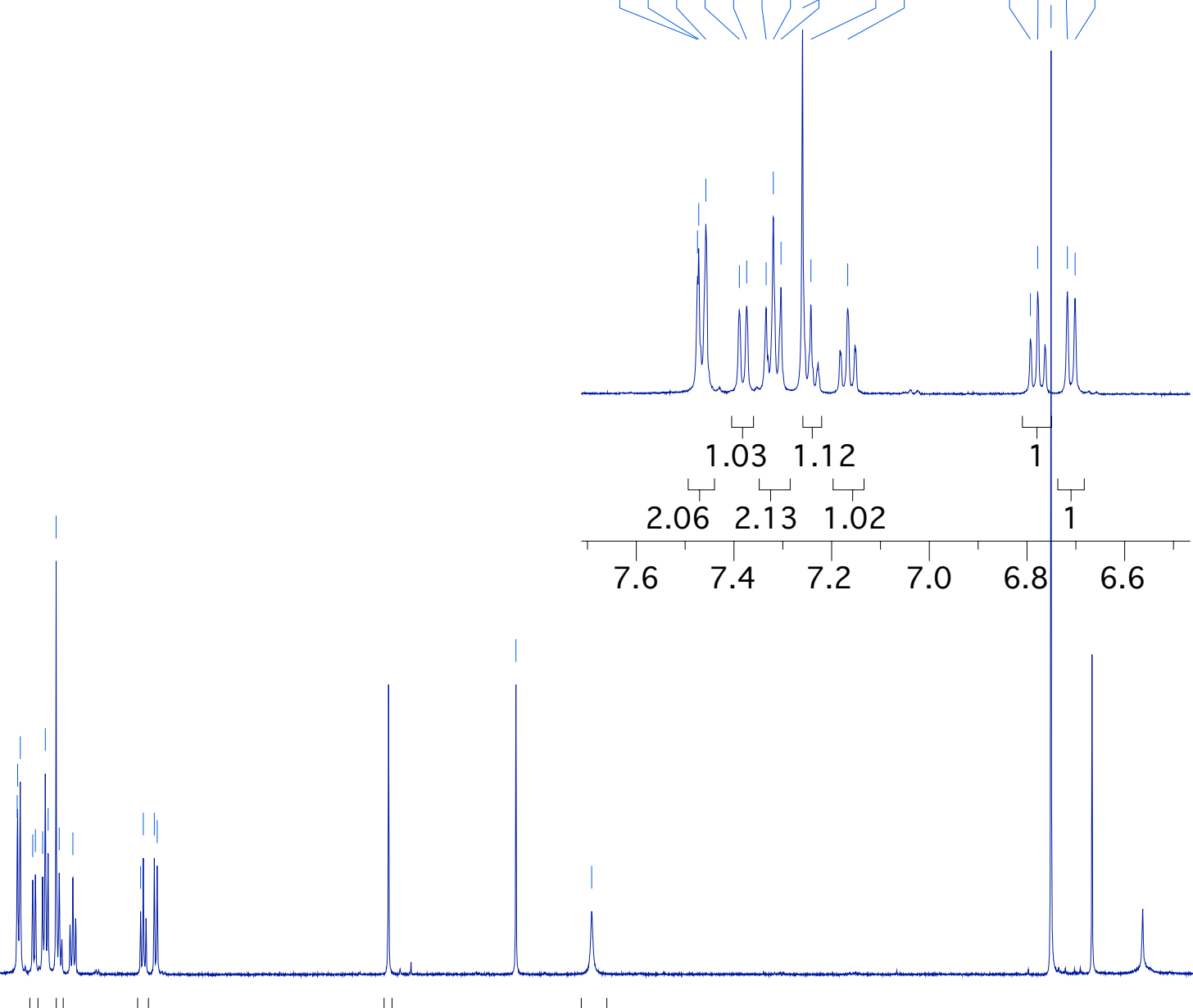

प

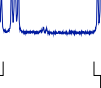

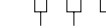

2.06131 .02

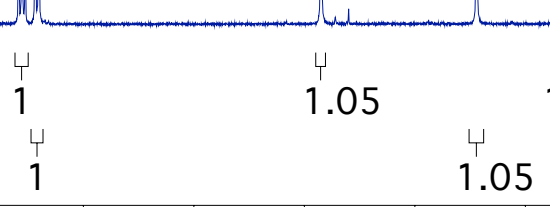

1.03

1.05

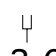

3.07 


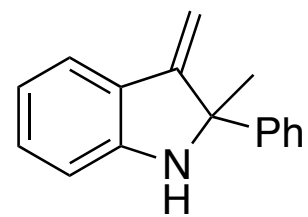

$37 c$ 


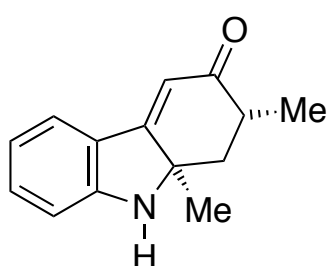

46

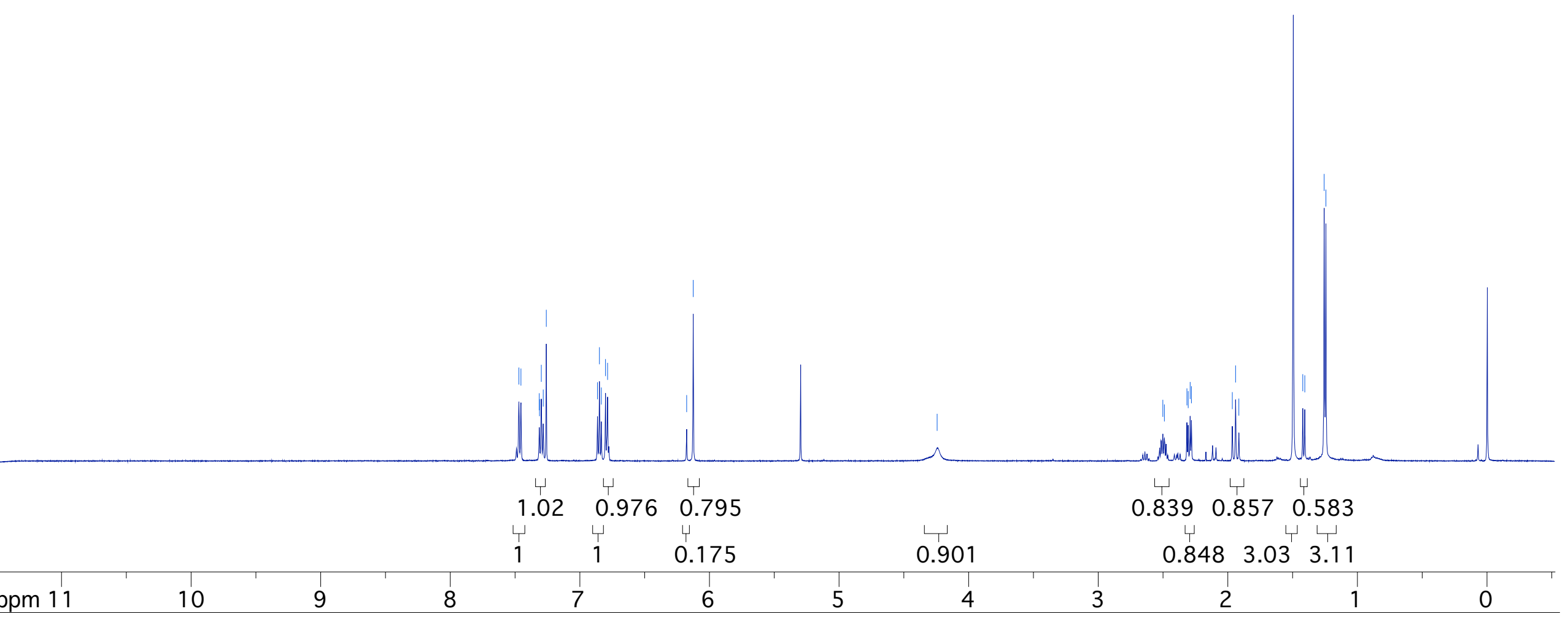




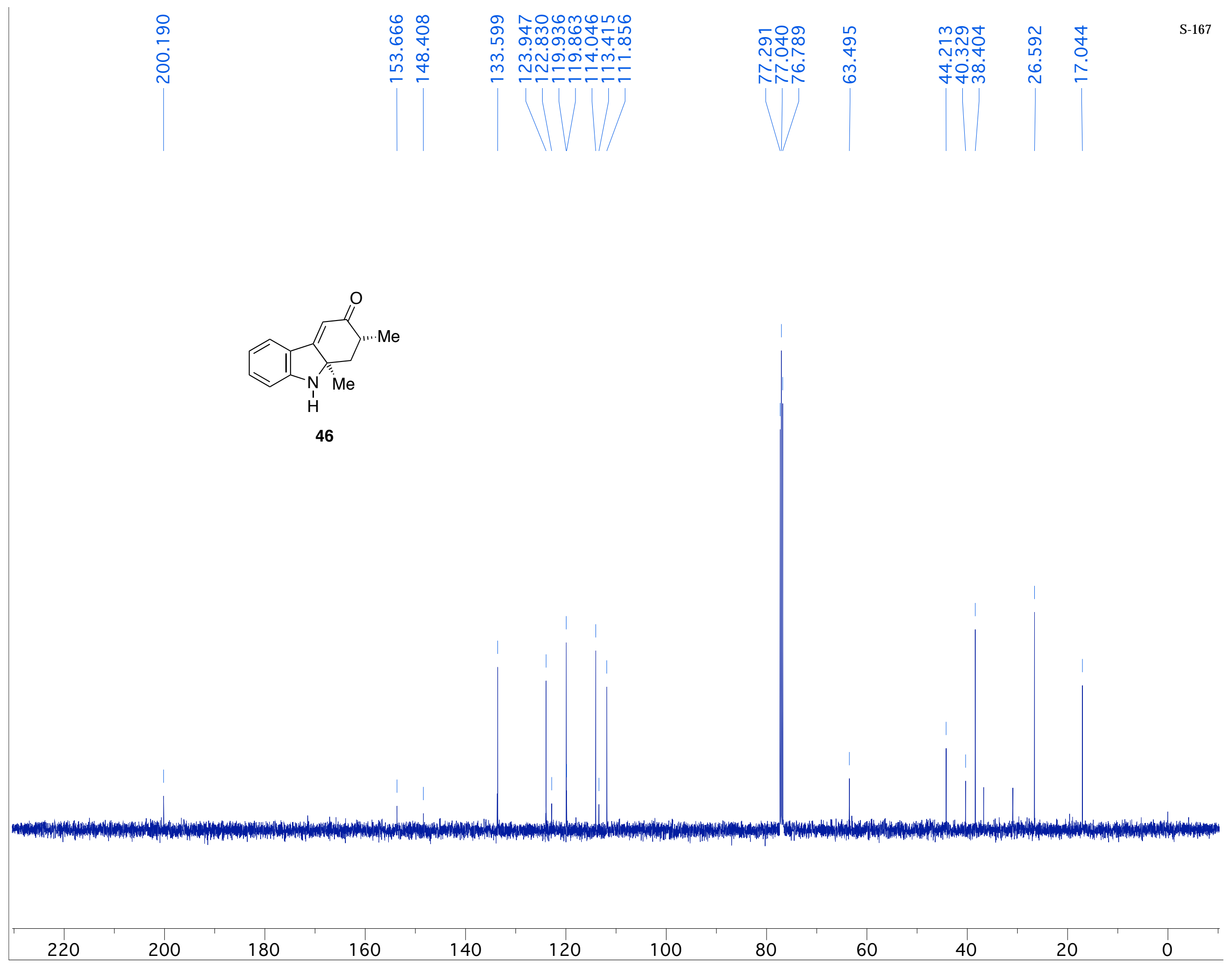




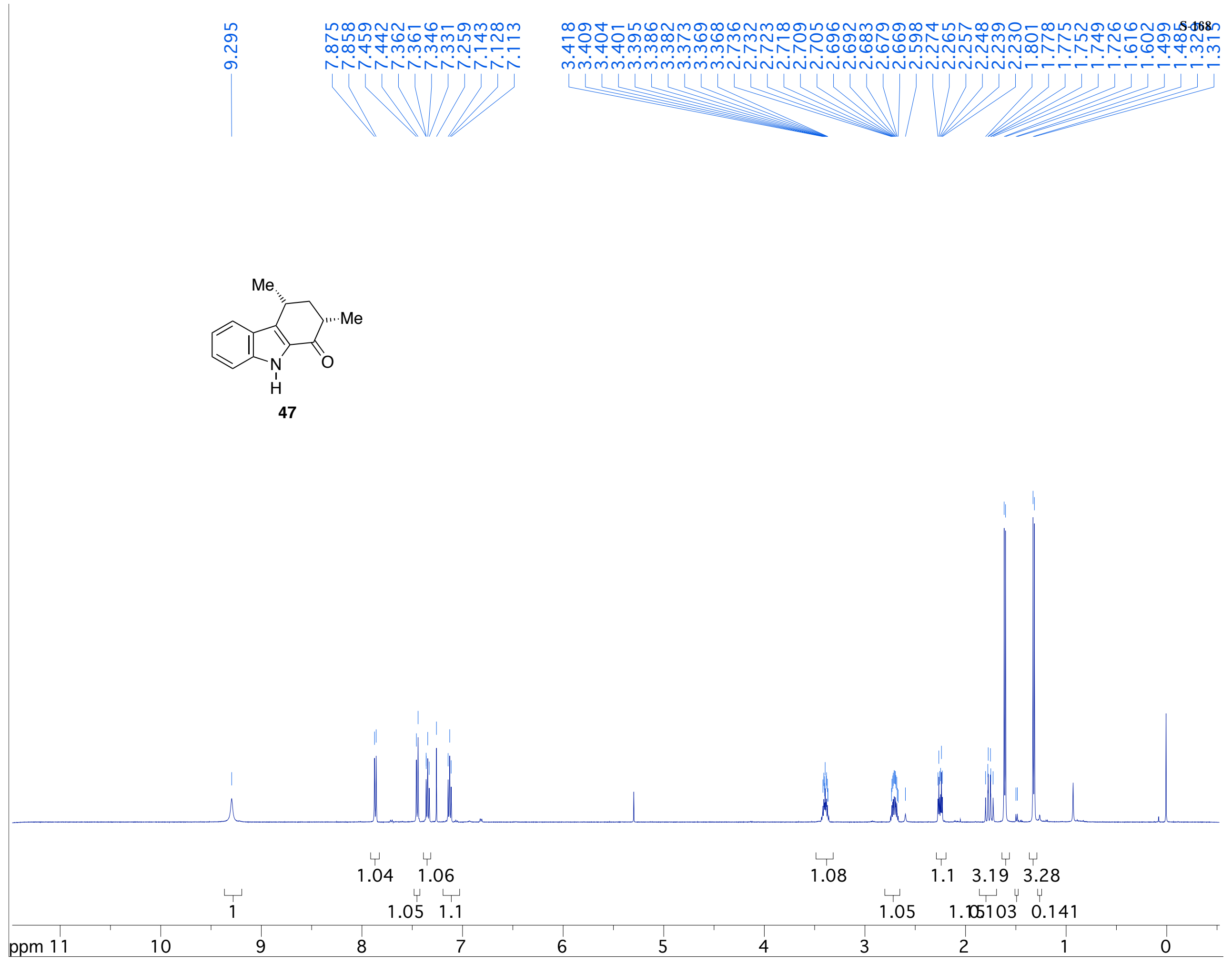




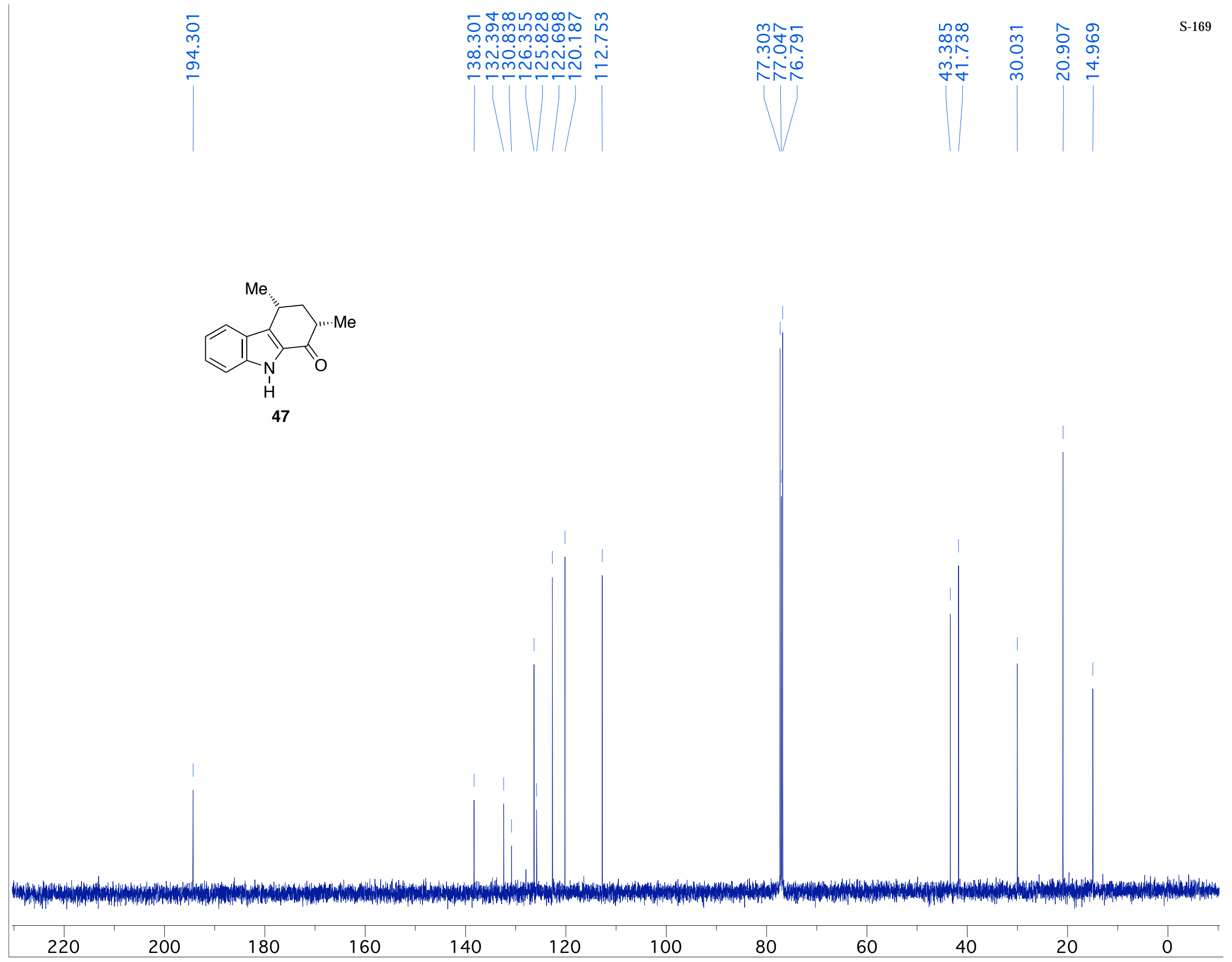




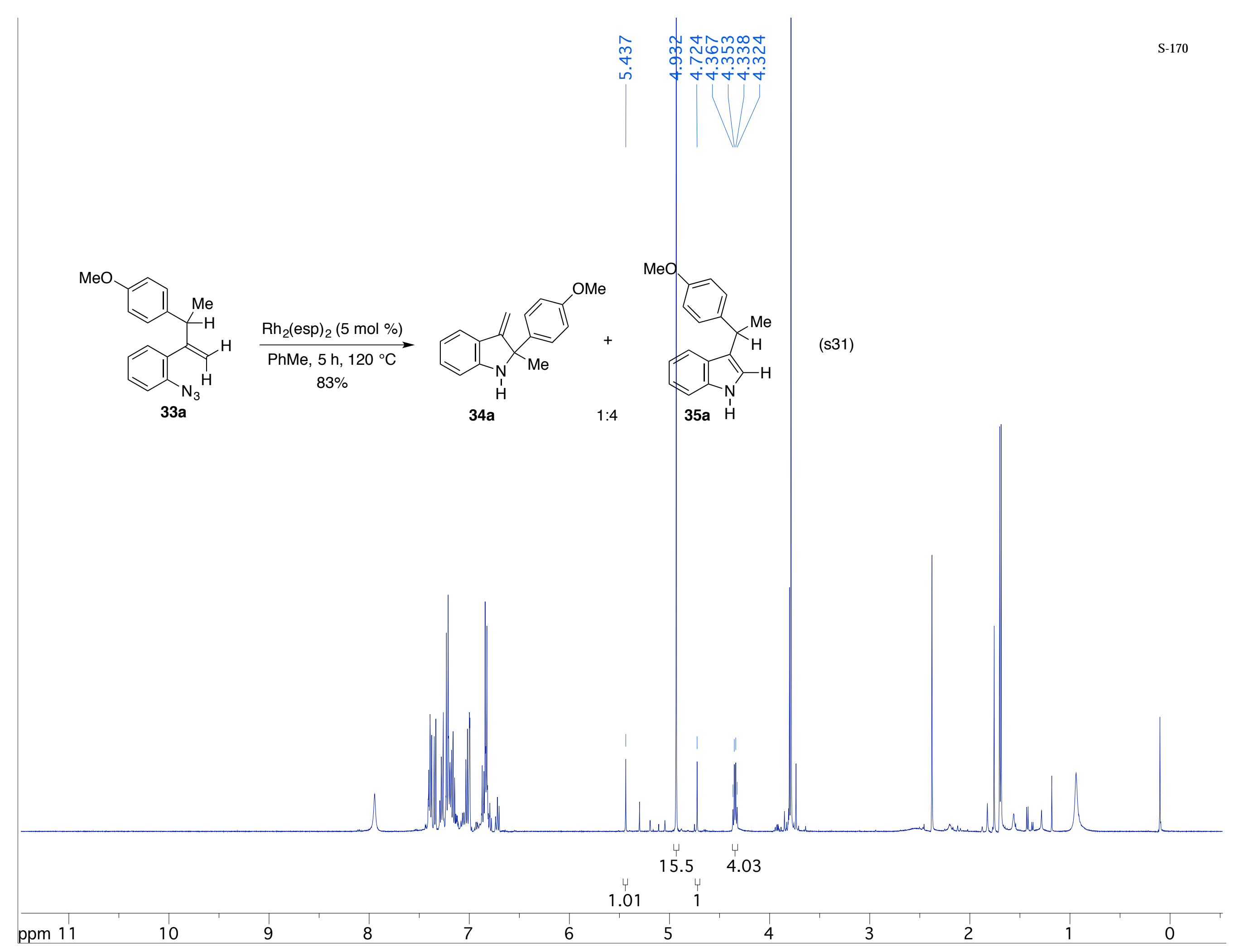




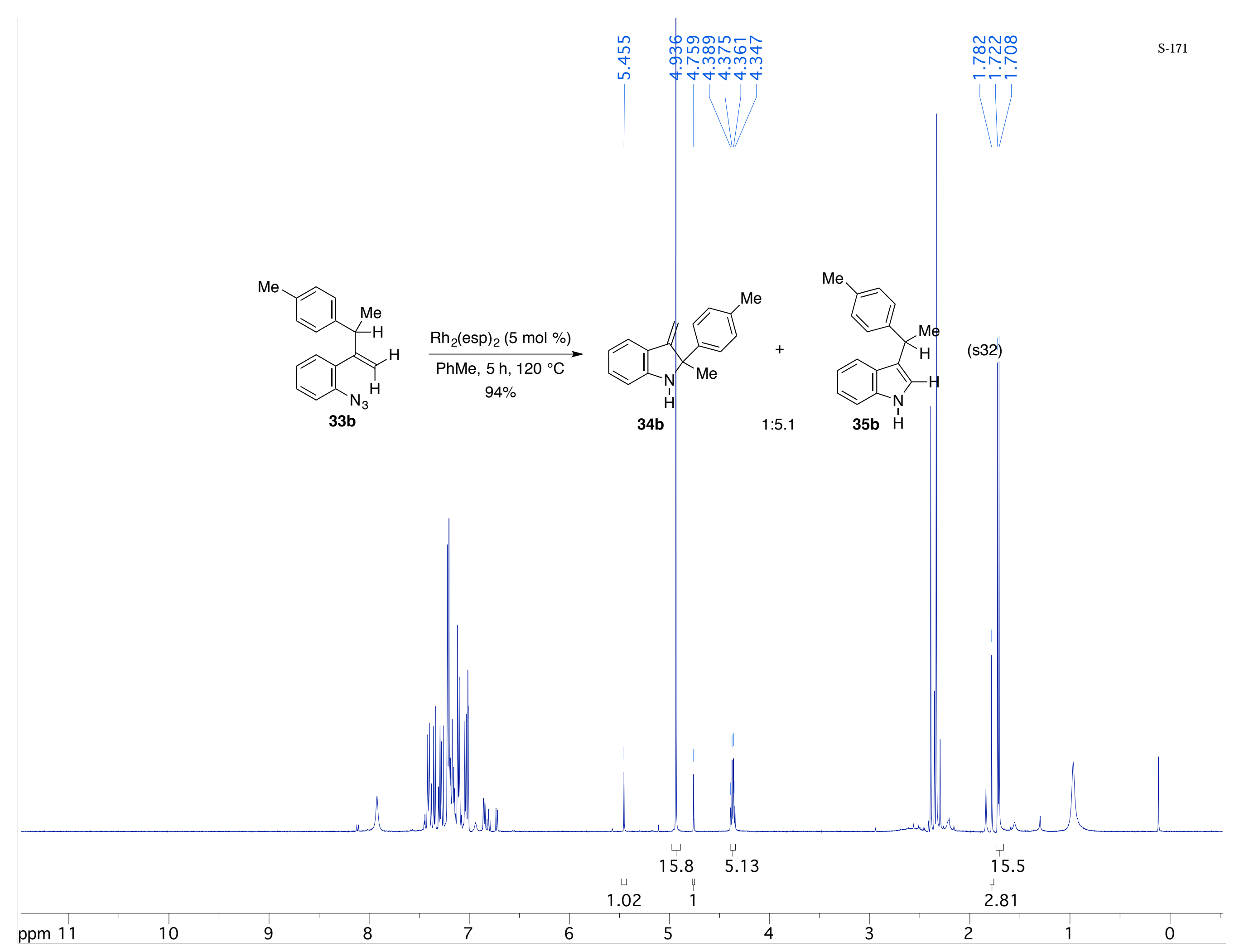




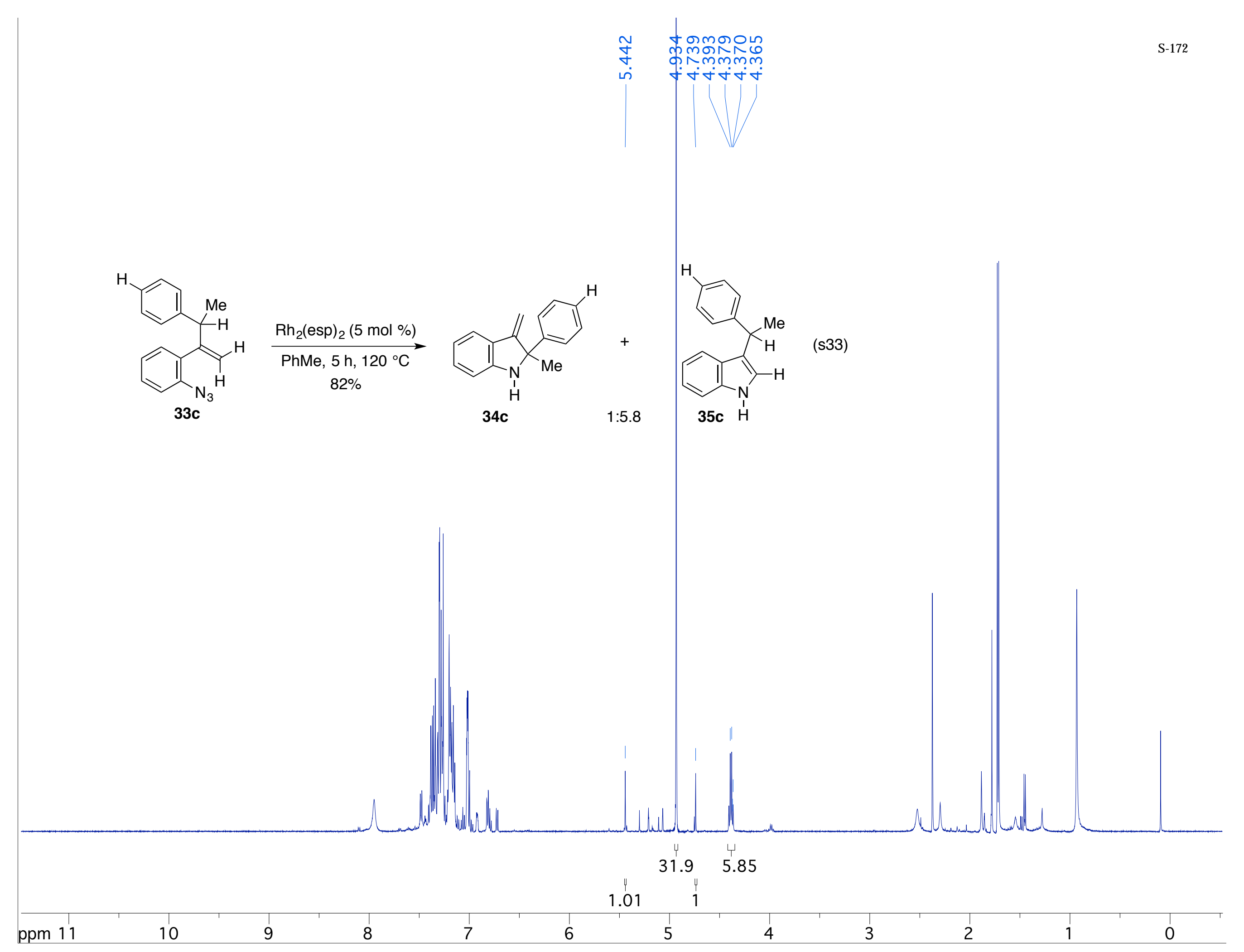




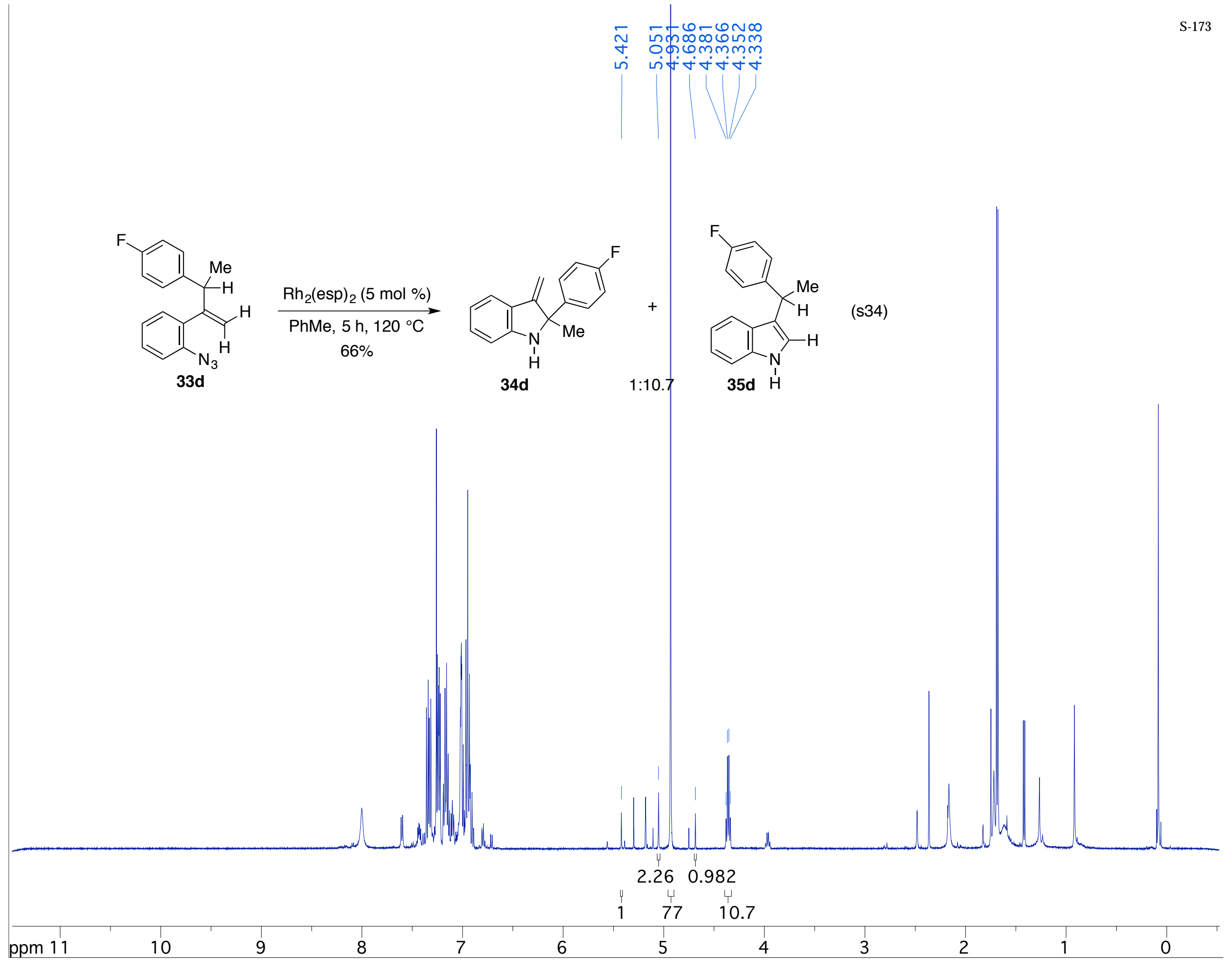




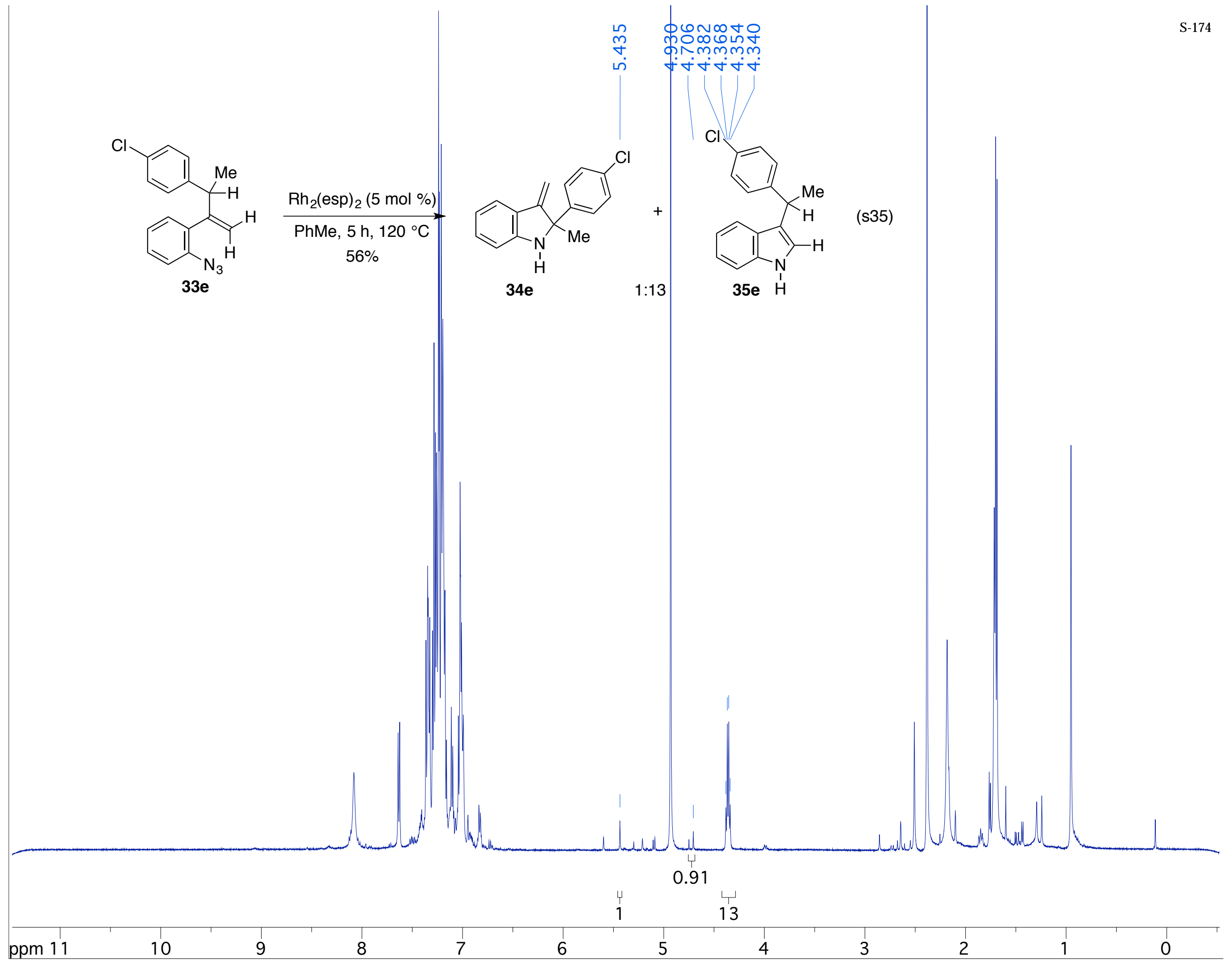




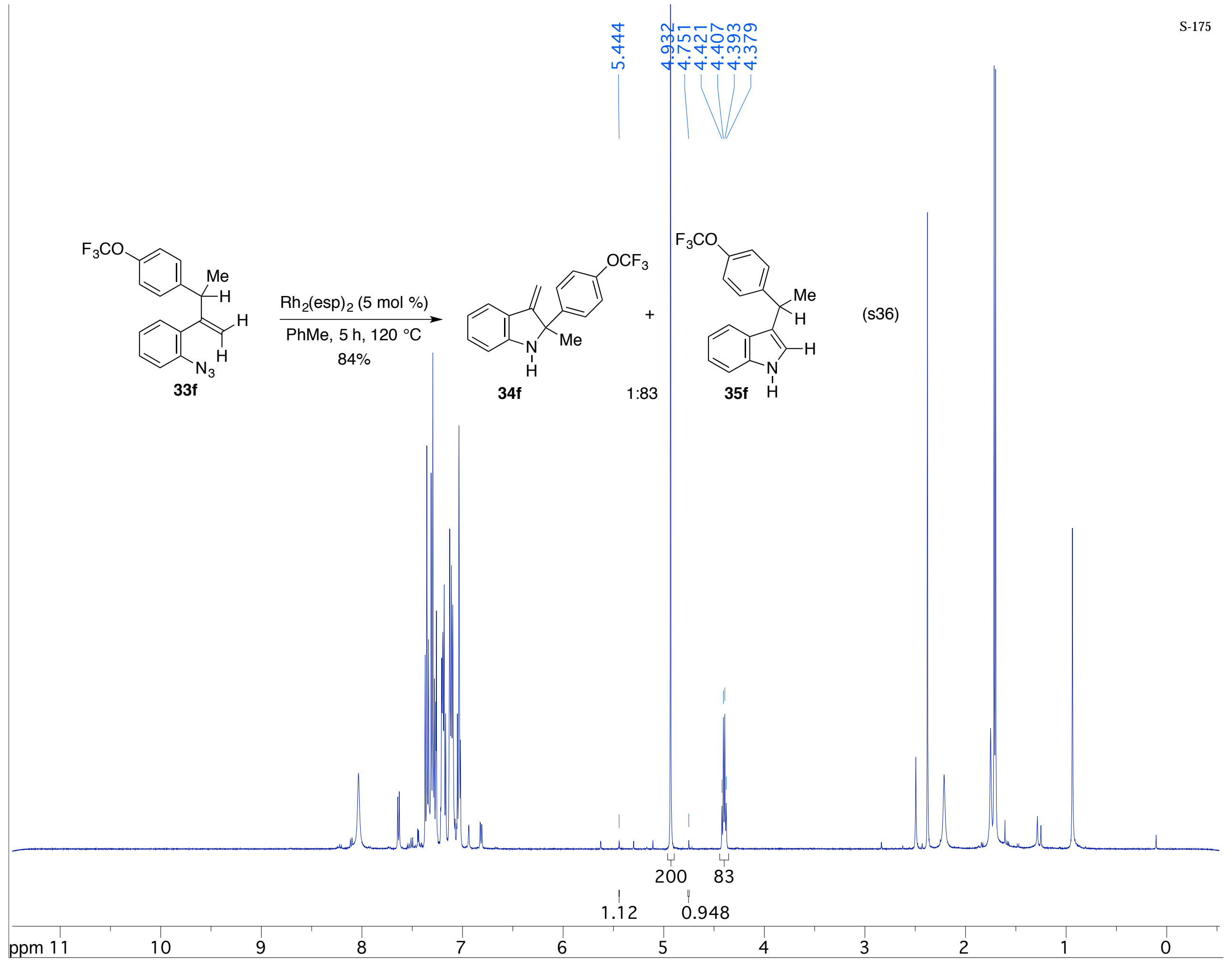




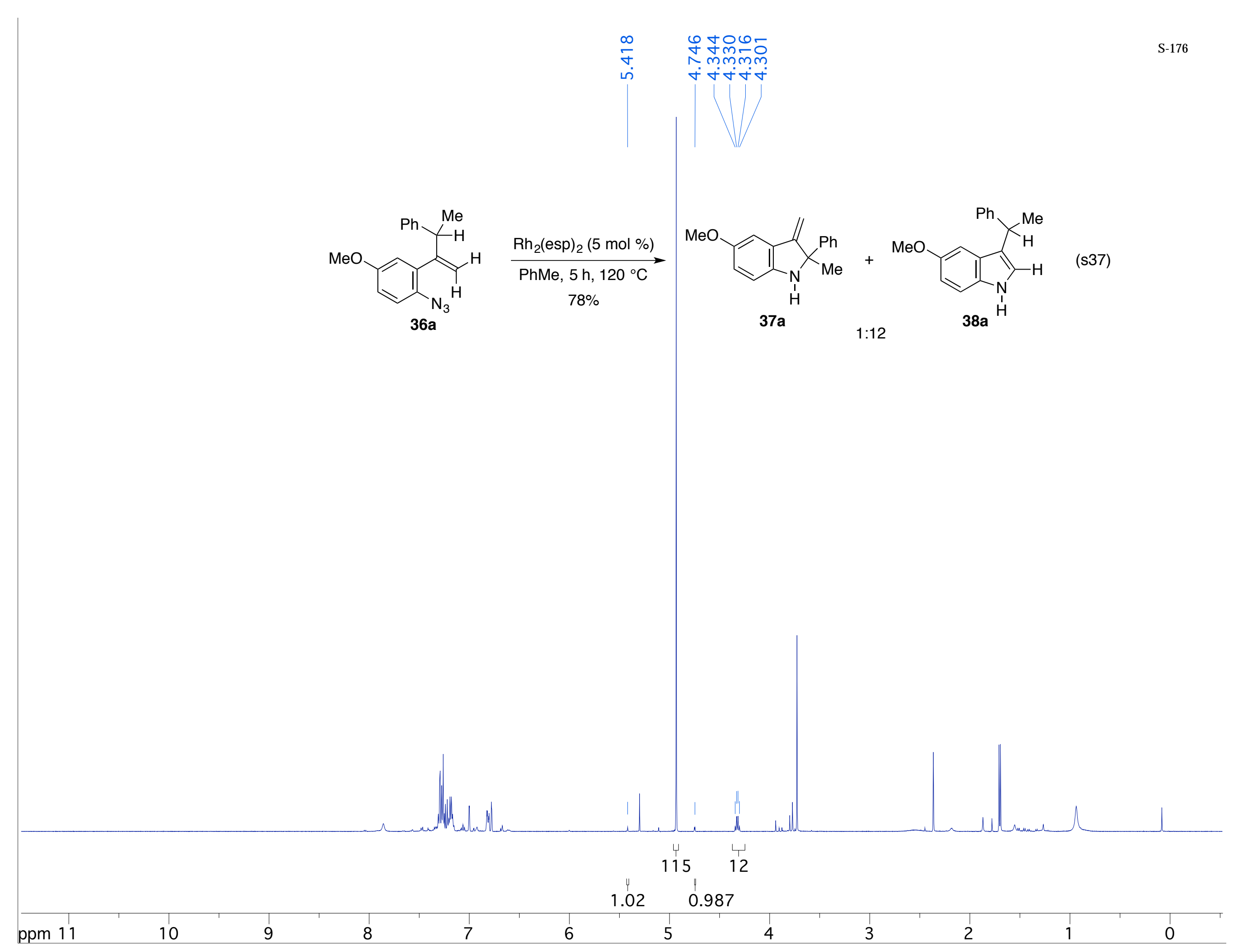


<smiles>Cc1ccc(N)c(/C(=C/c2ccccc2)C(C)(C)c2ccccc2)c1</smiles>

36b

\section{$\mathrm{Rh}_{2}(\mathrm{esp})_{2}(5 \mathrm{~mol} \%)$} PhMe, $5 \mathrm{~h}, 120^{\circ} \mathrm{C}$ $79 \%$

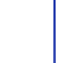

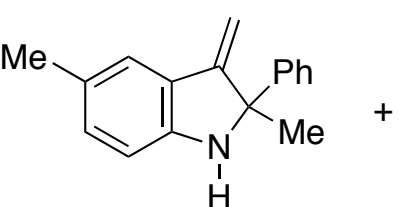

37b

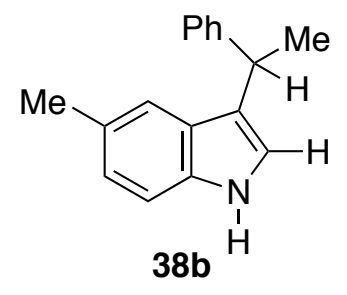

$: 7.2$ 


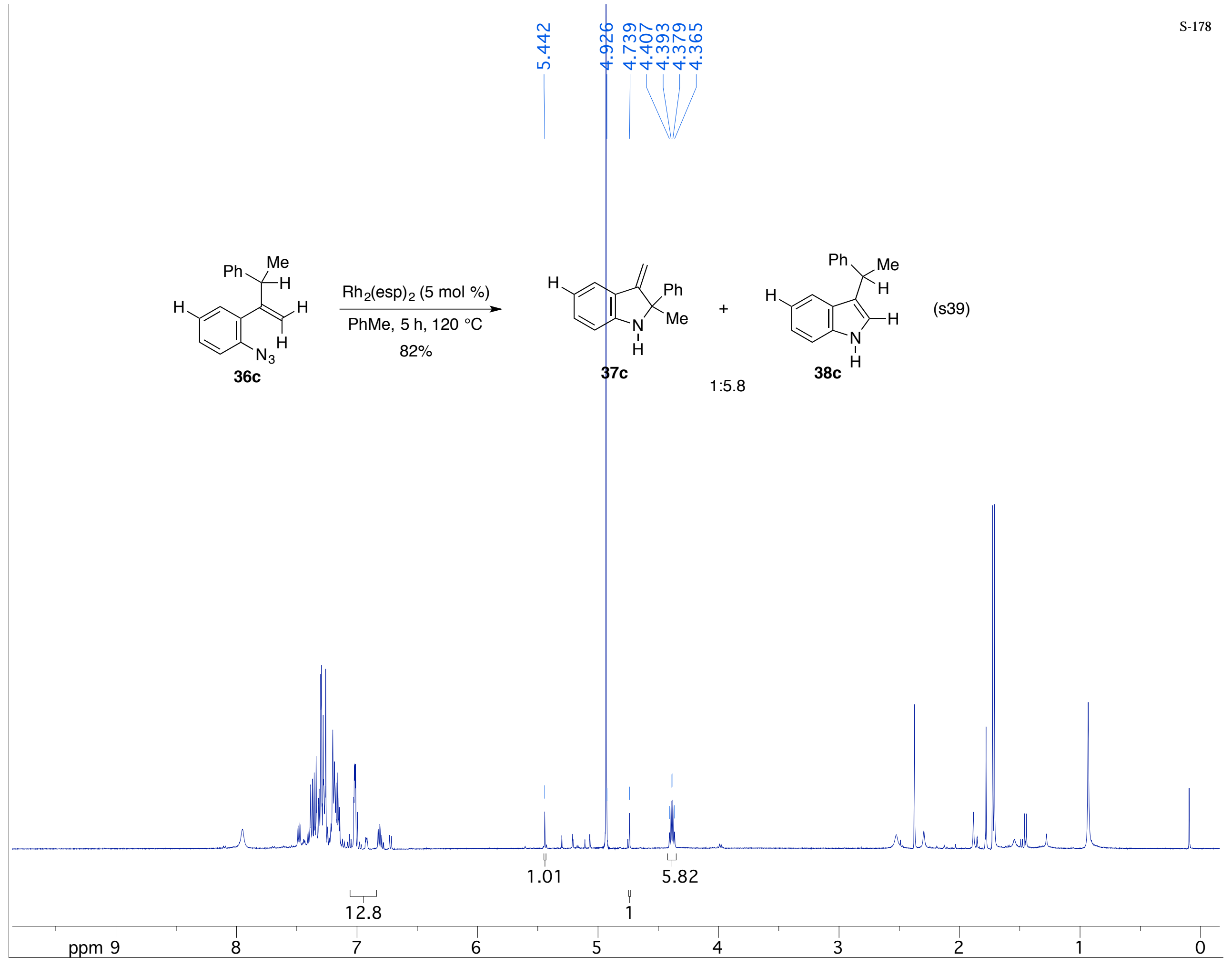




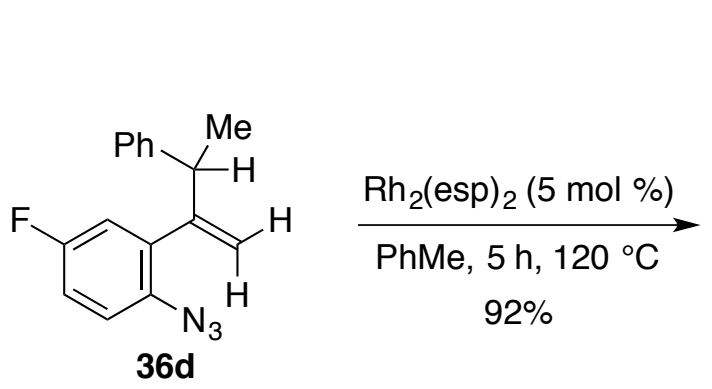

36d
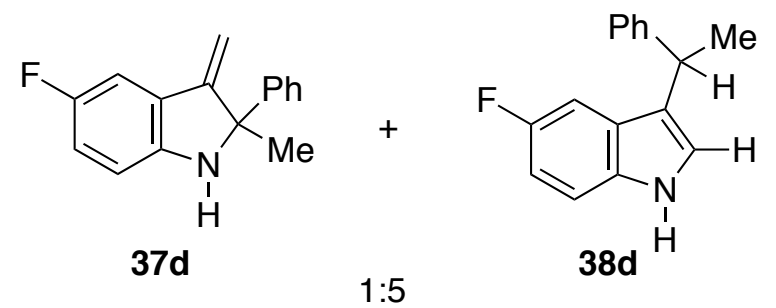

(s40)

$1: 5$

38d

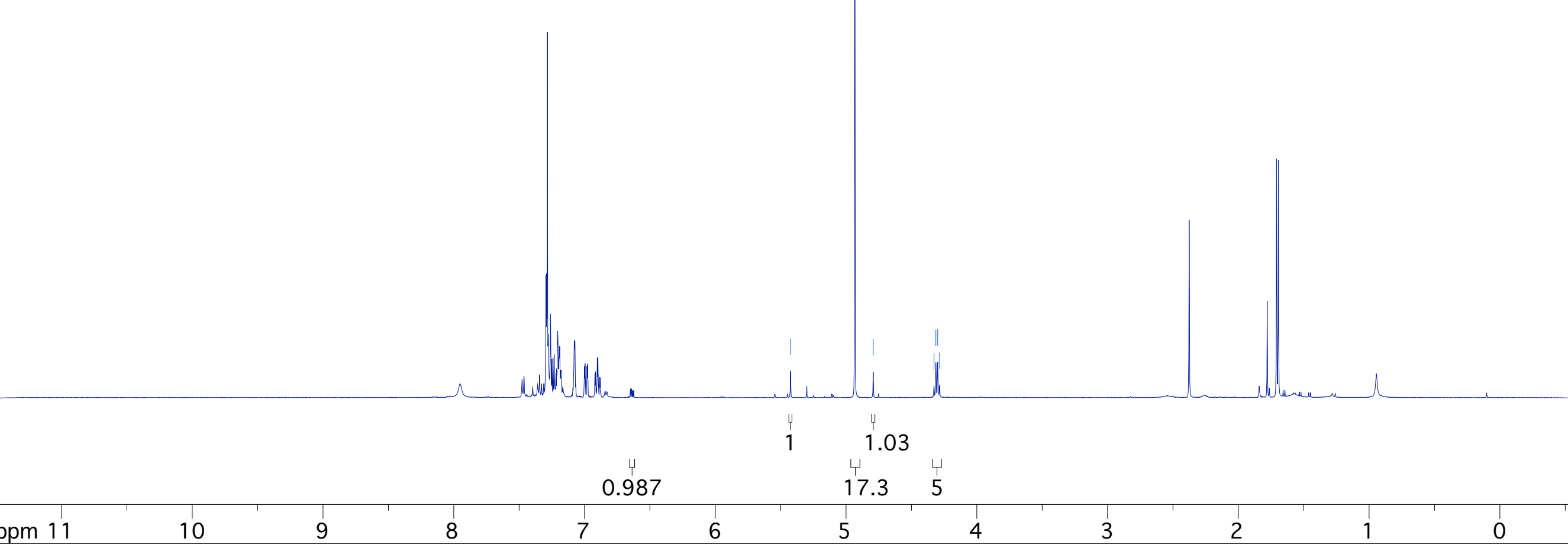




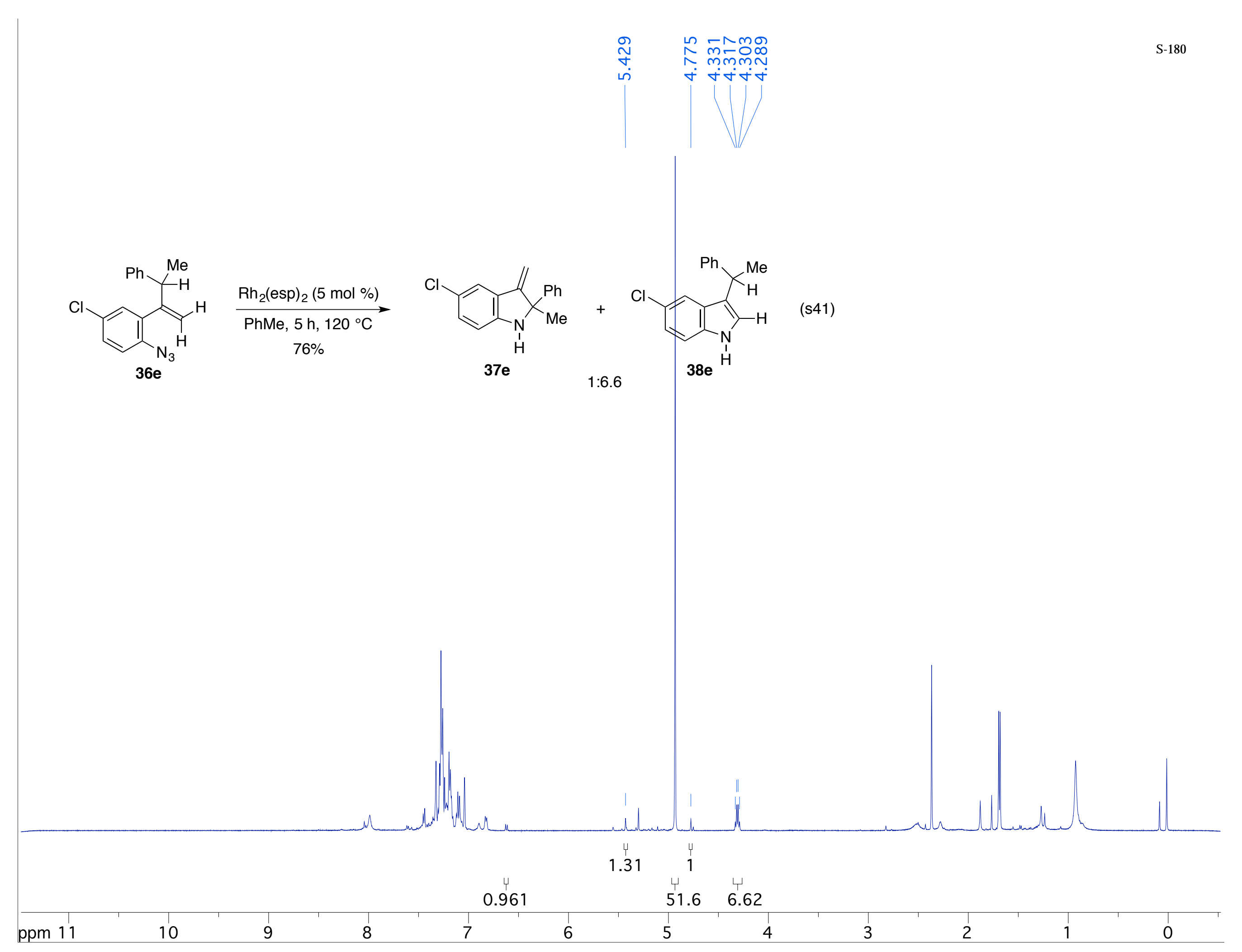




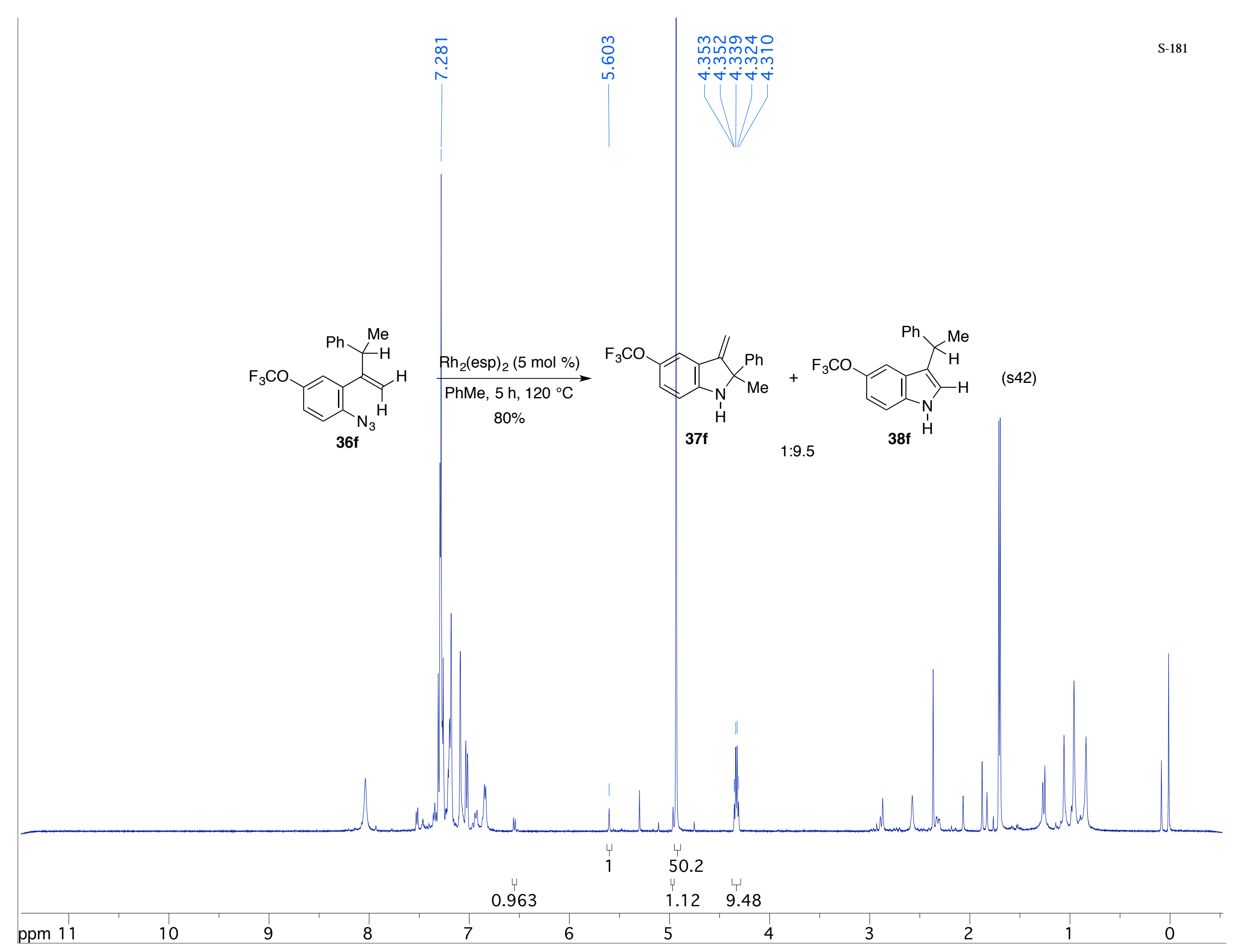




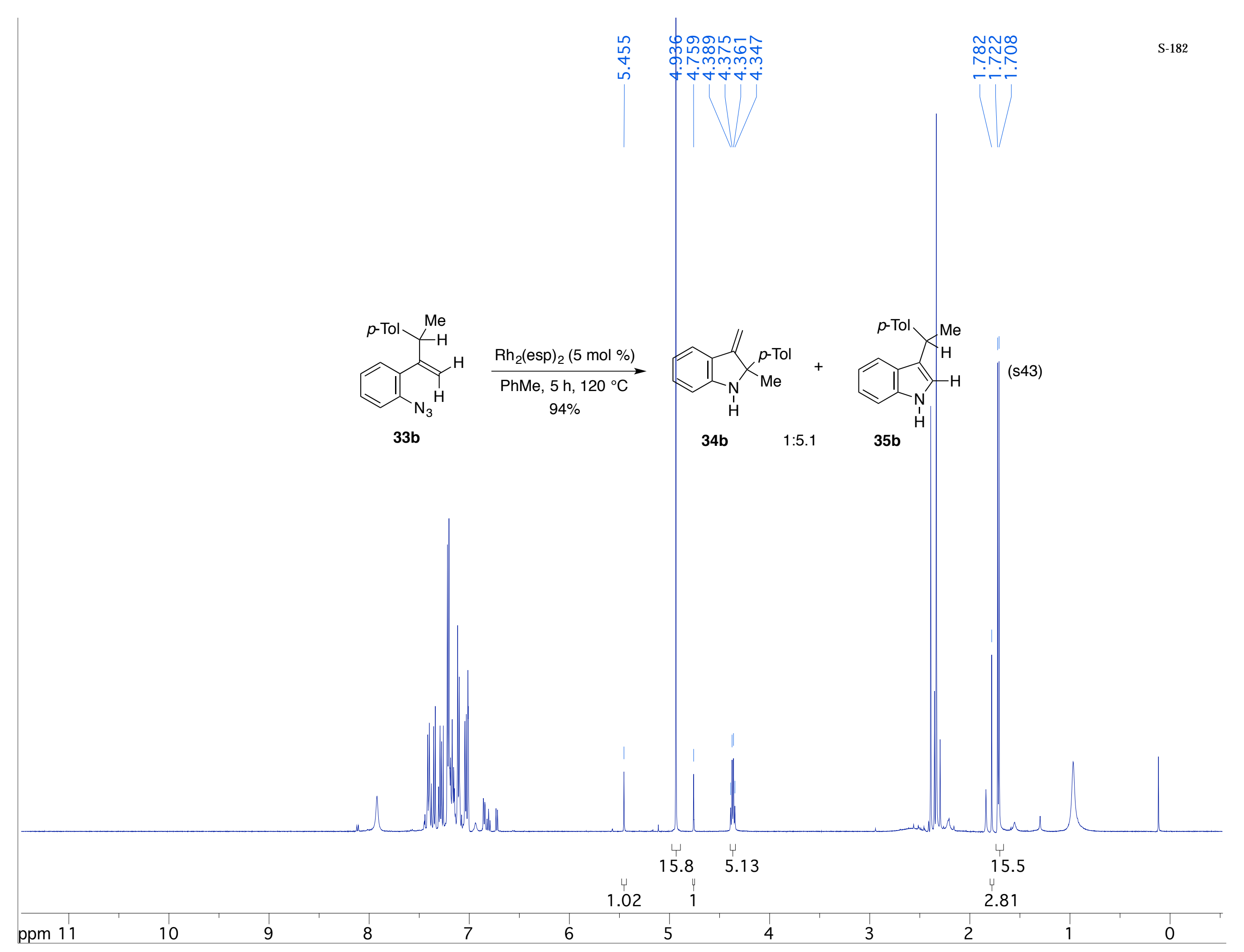




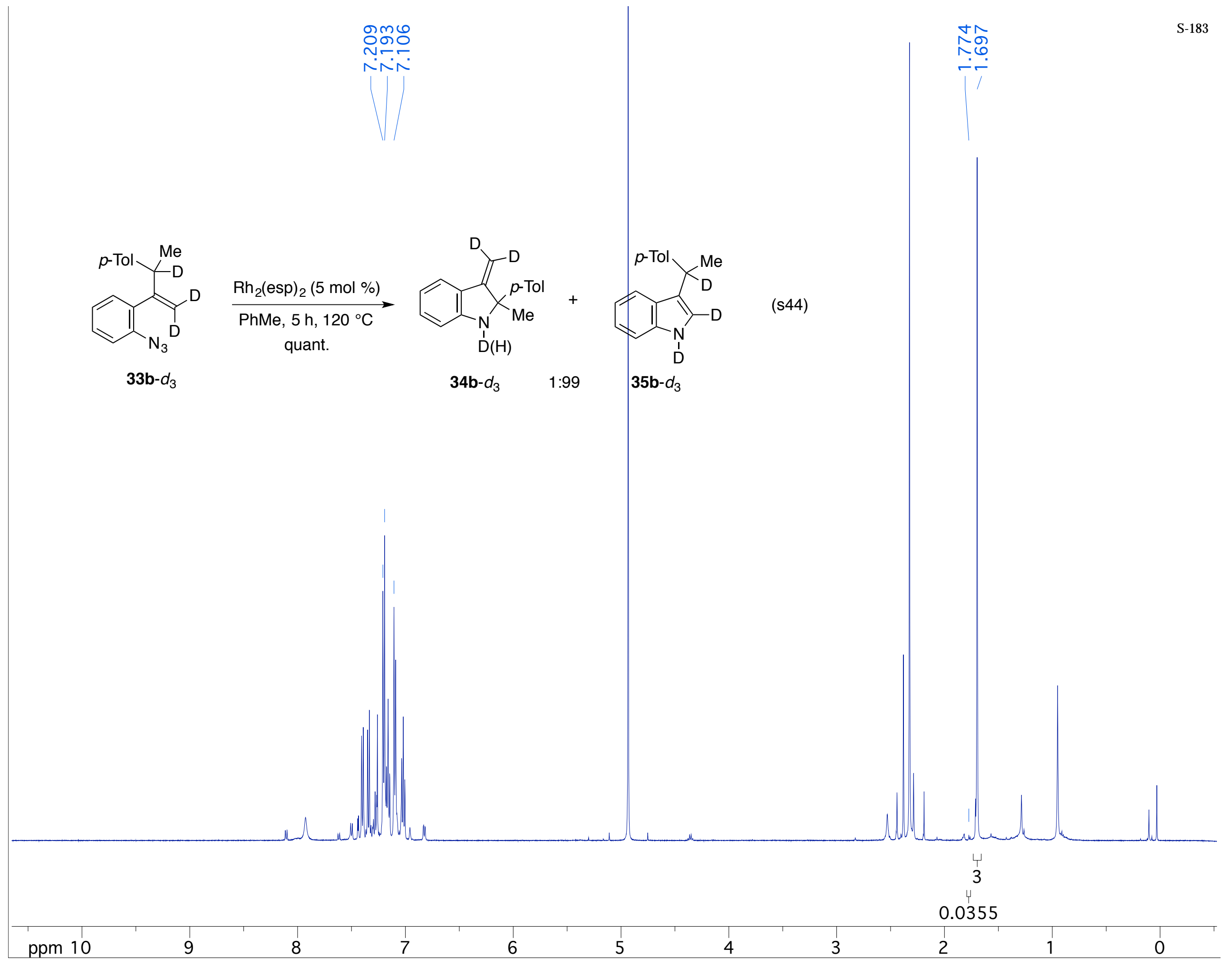




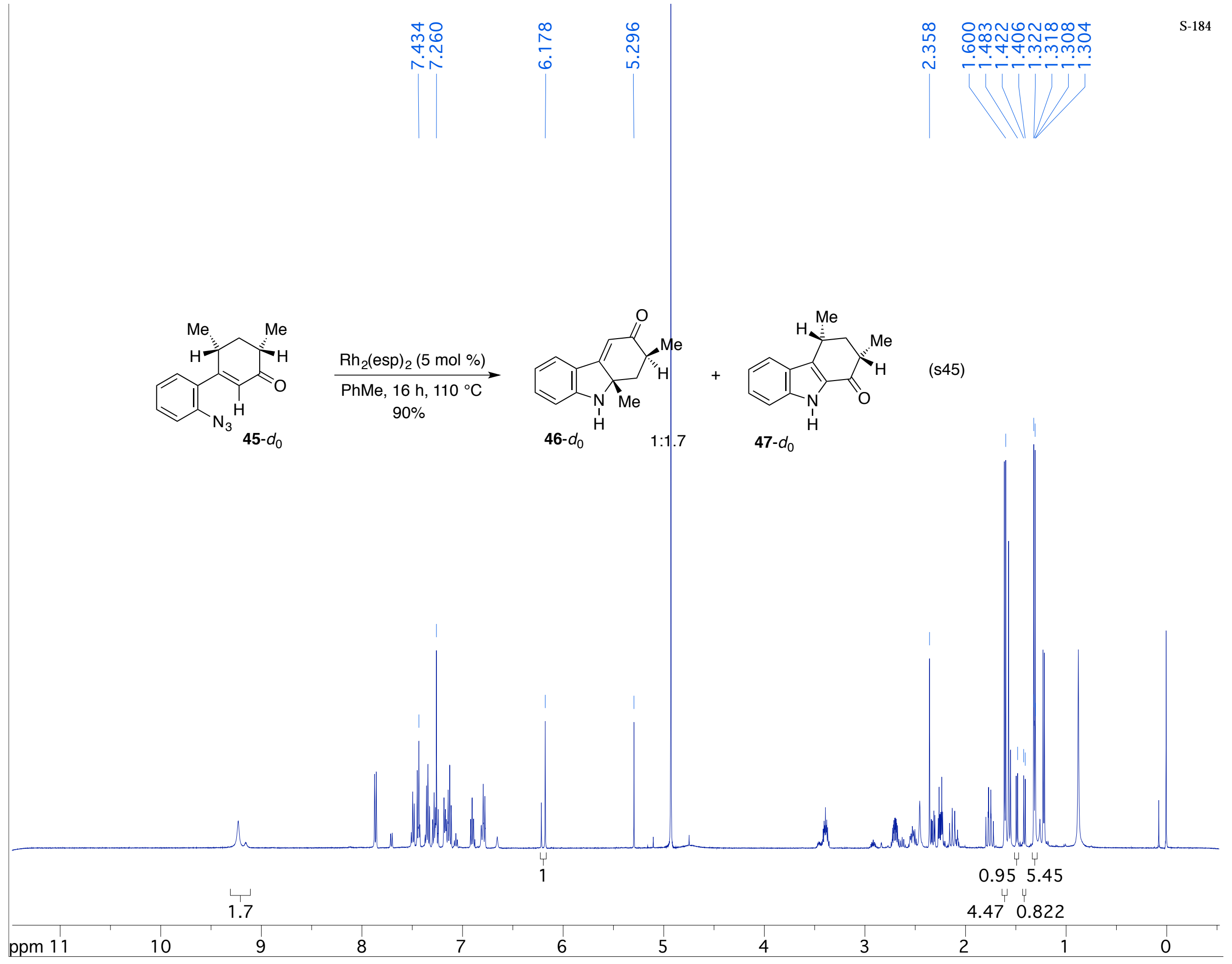




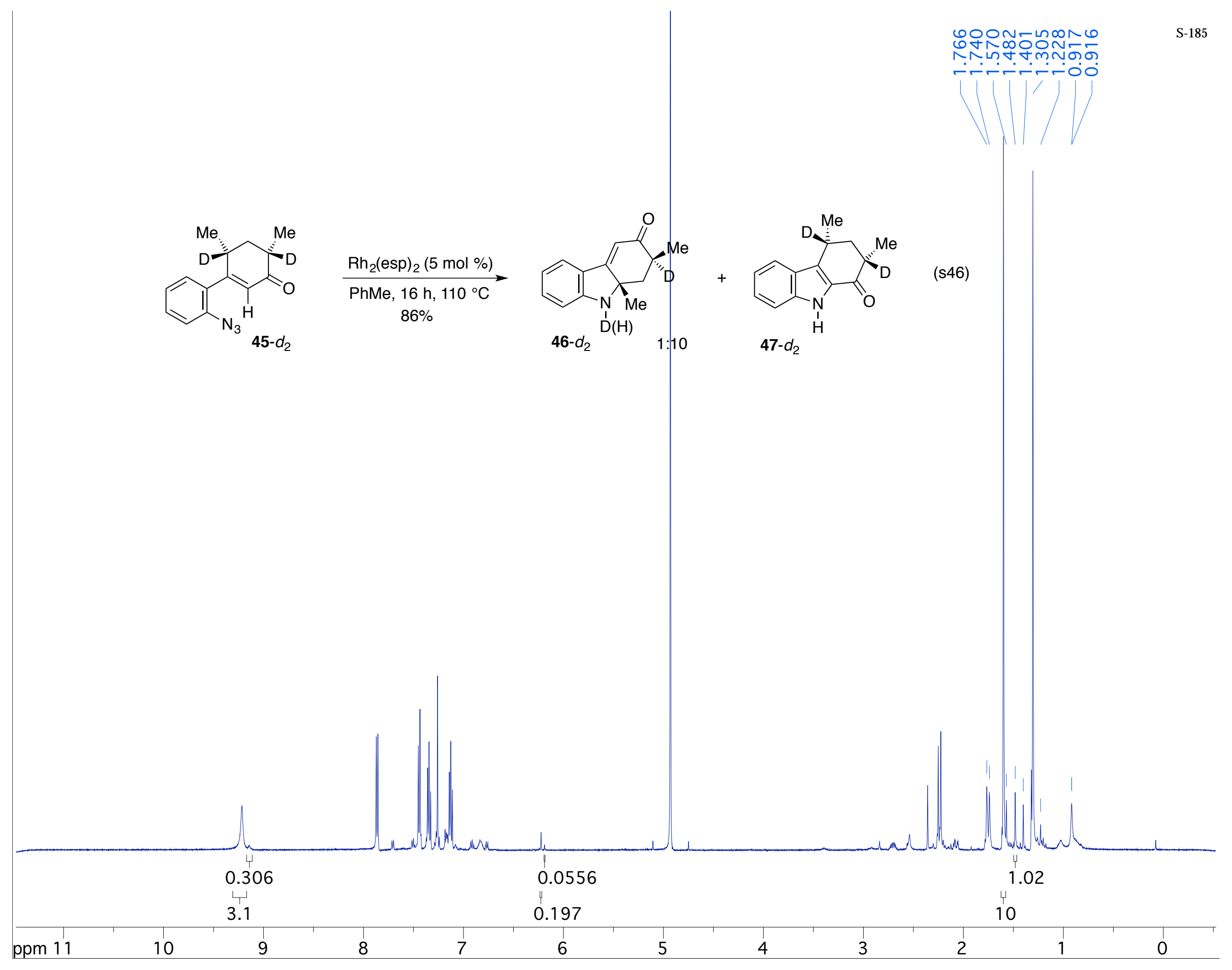




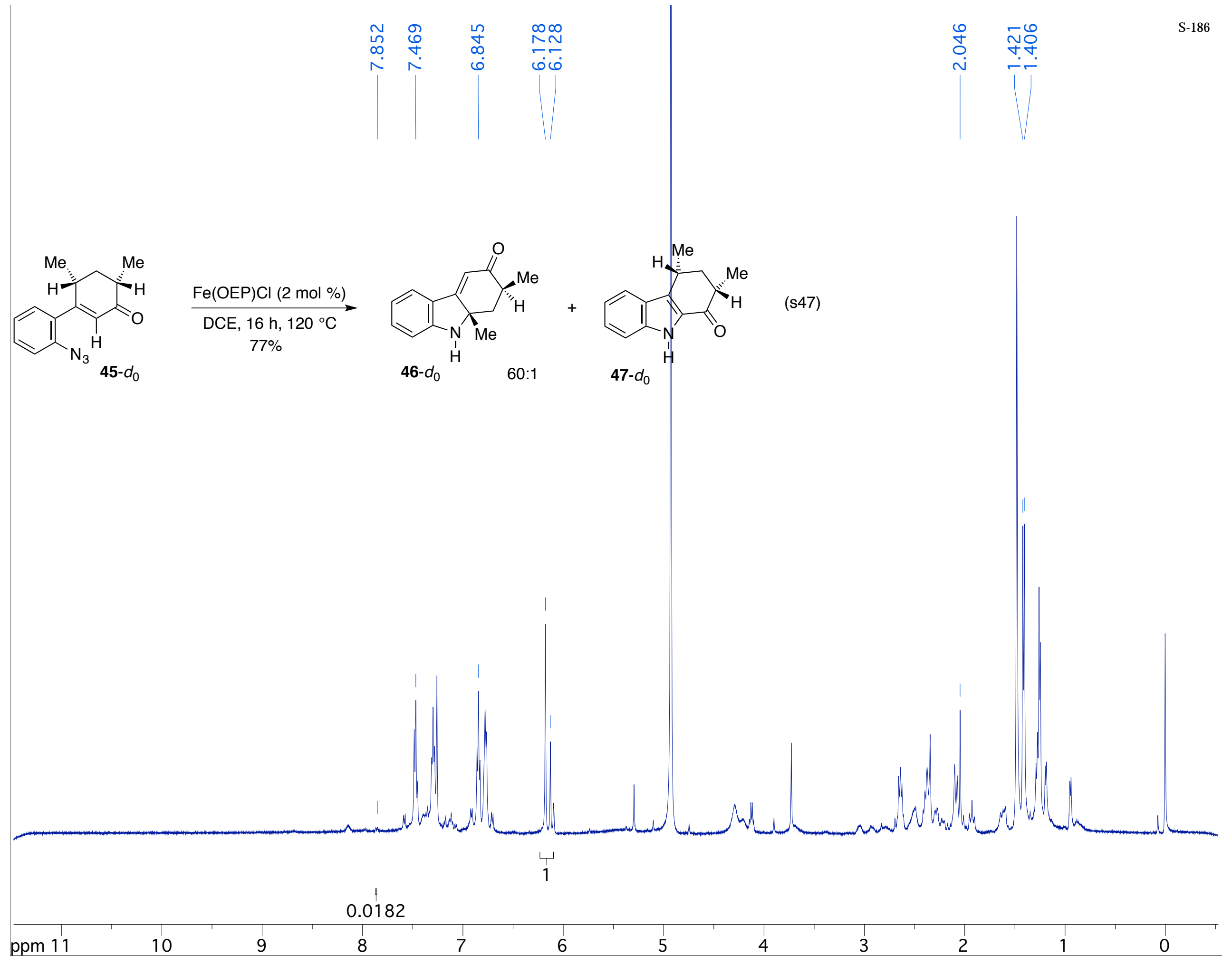




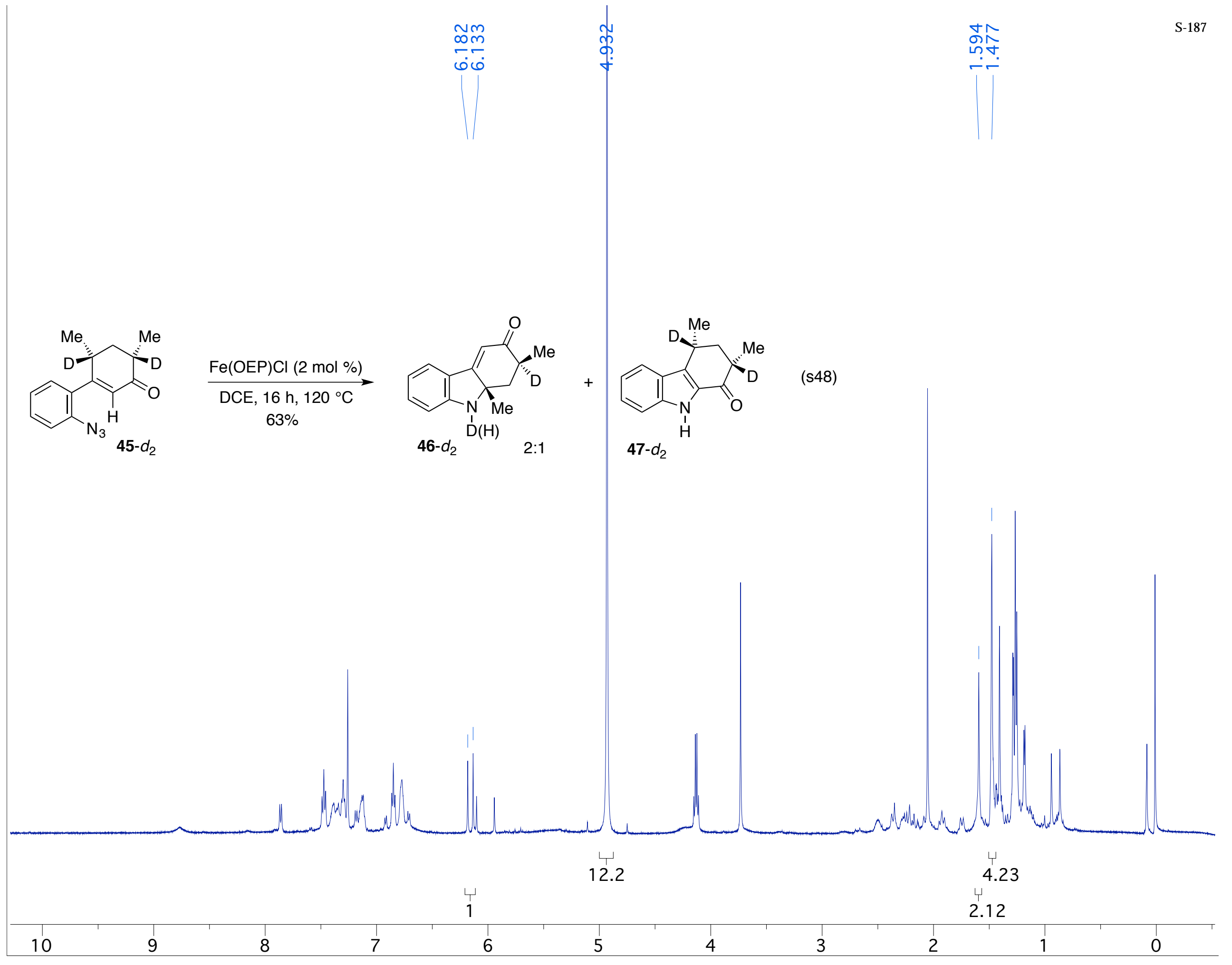




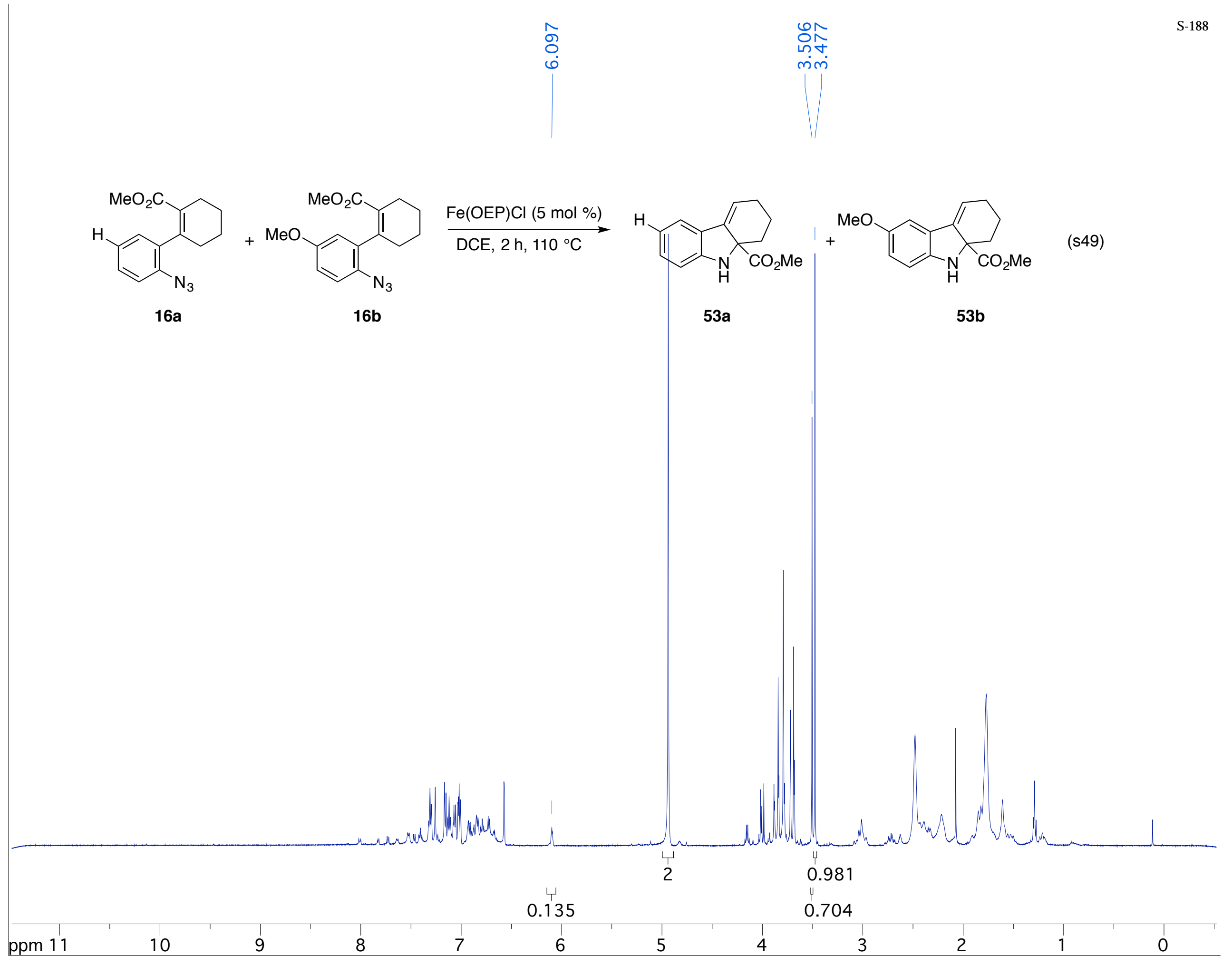


กัะ

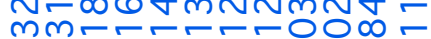

NNNNNNÑ́n

L l 1 1 1)

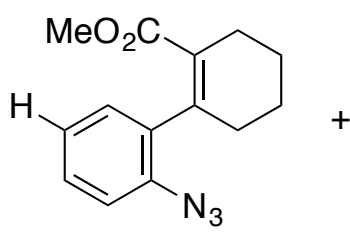

$16 a$

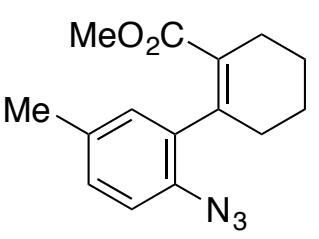

16c

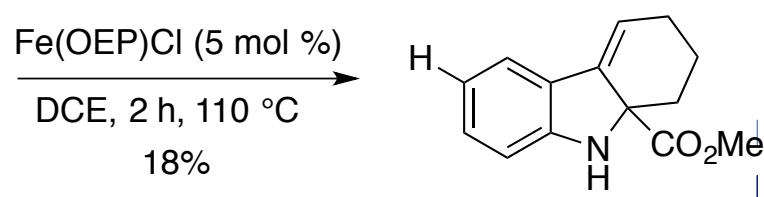

$53 a$

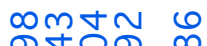

पmmo $N$

Ninin -

जंगंल 


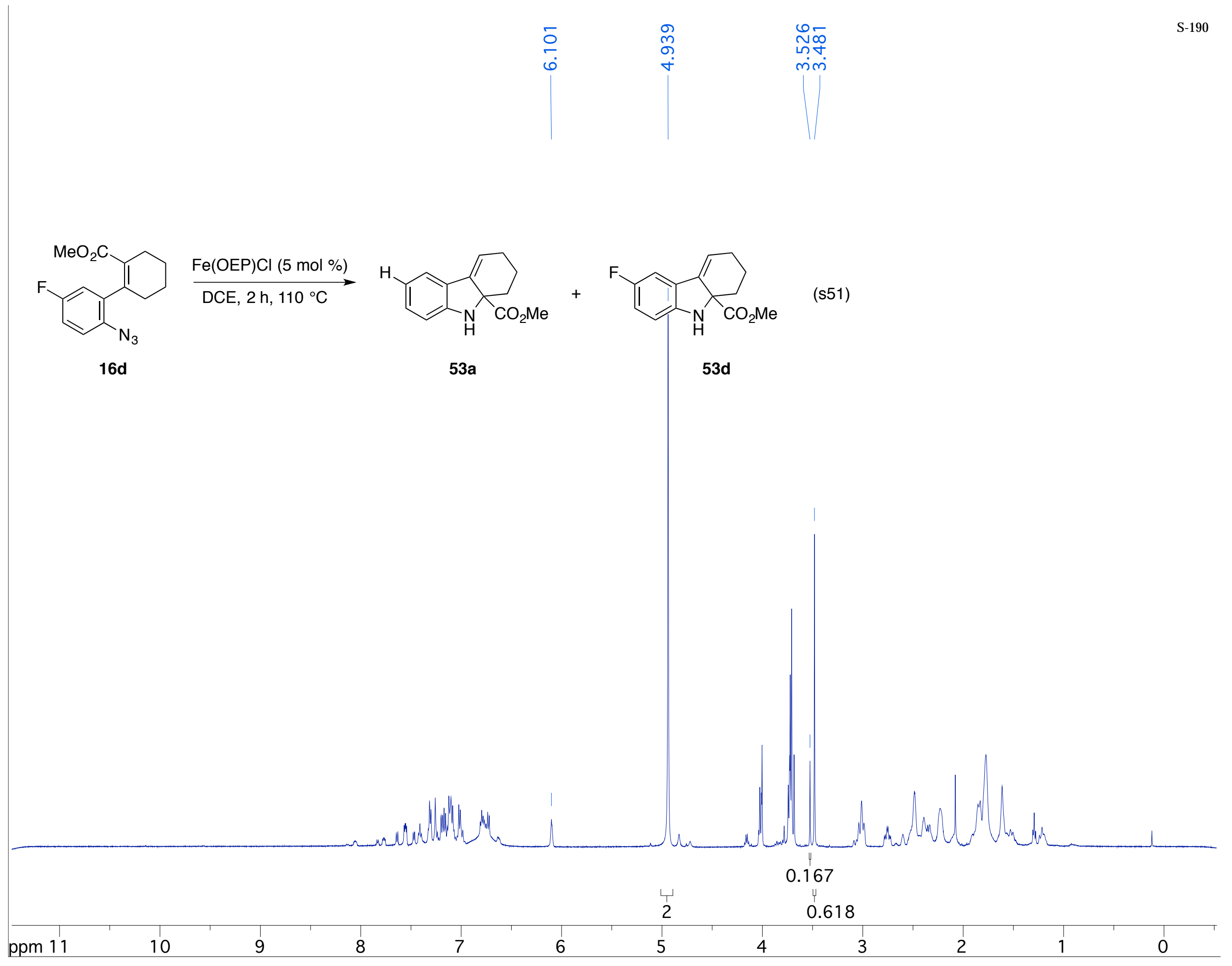




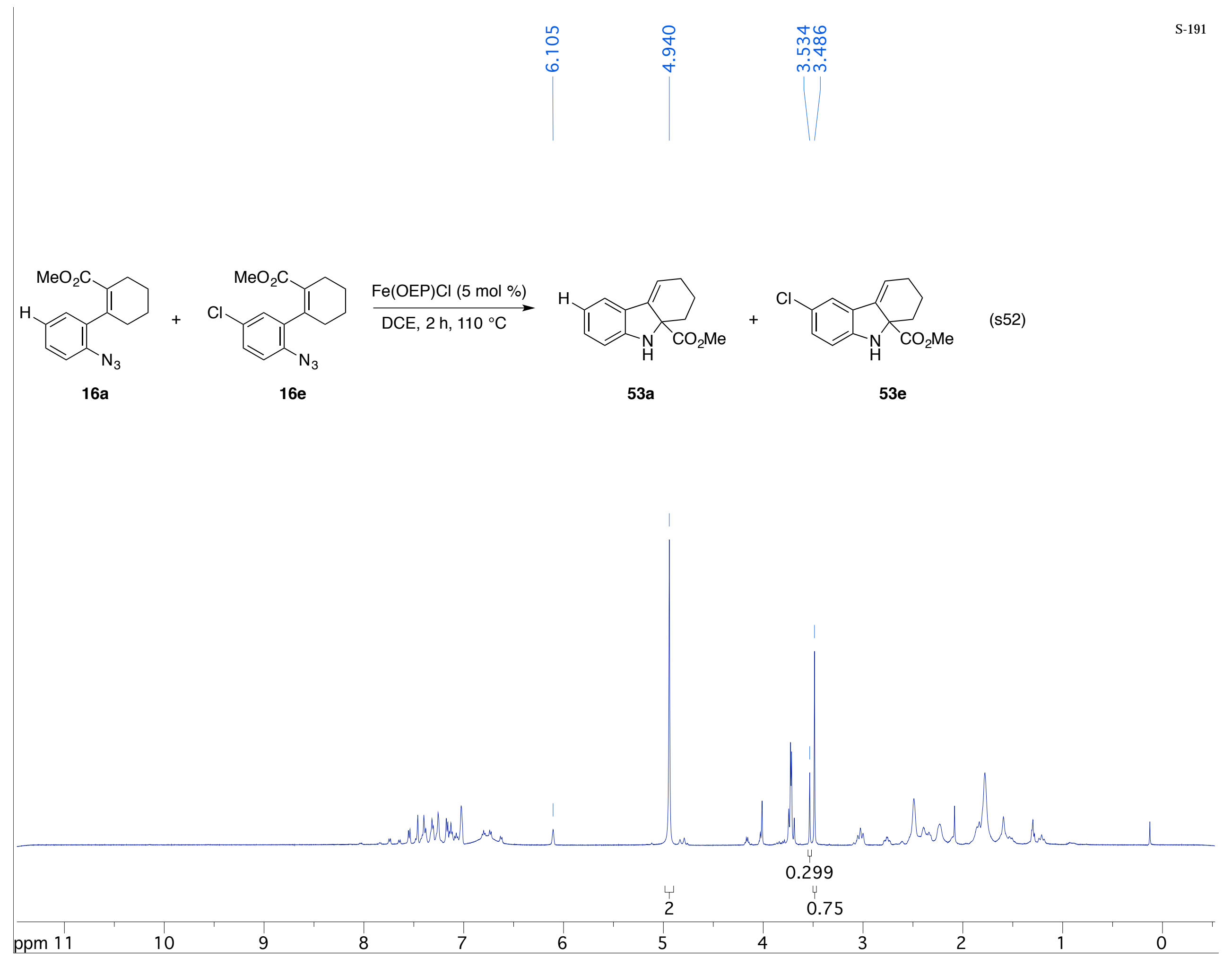




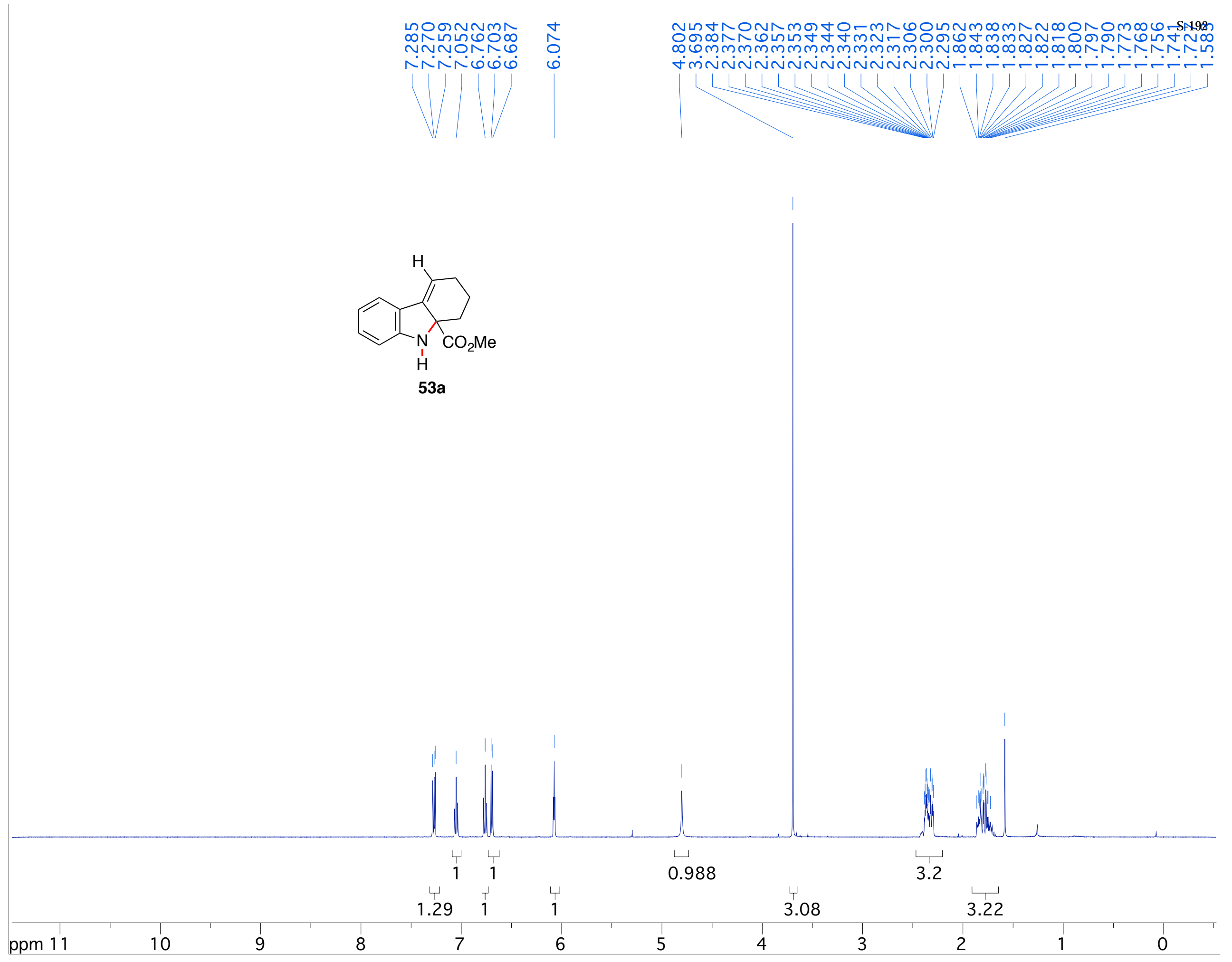




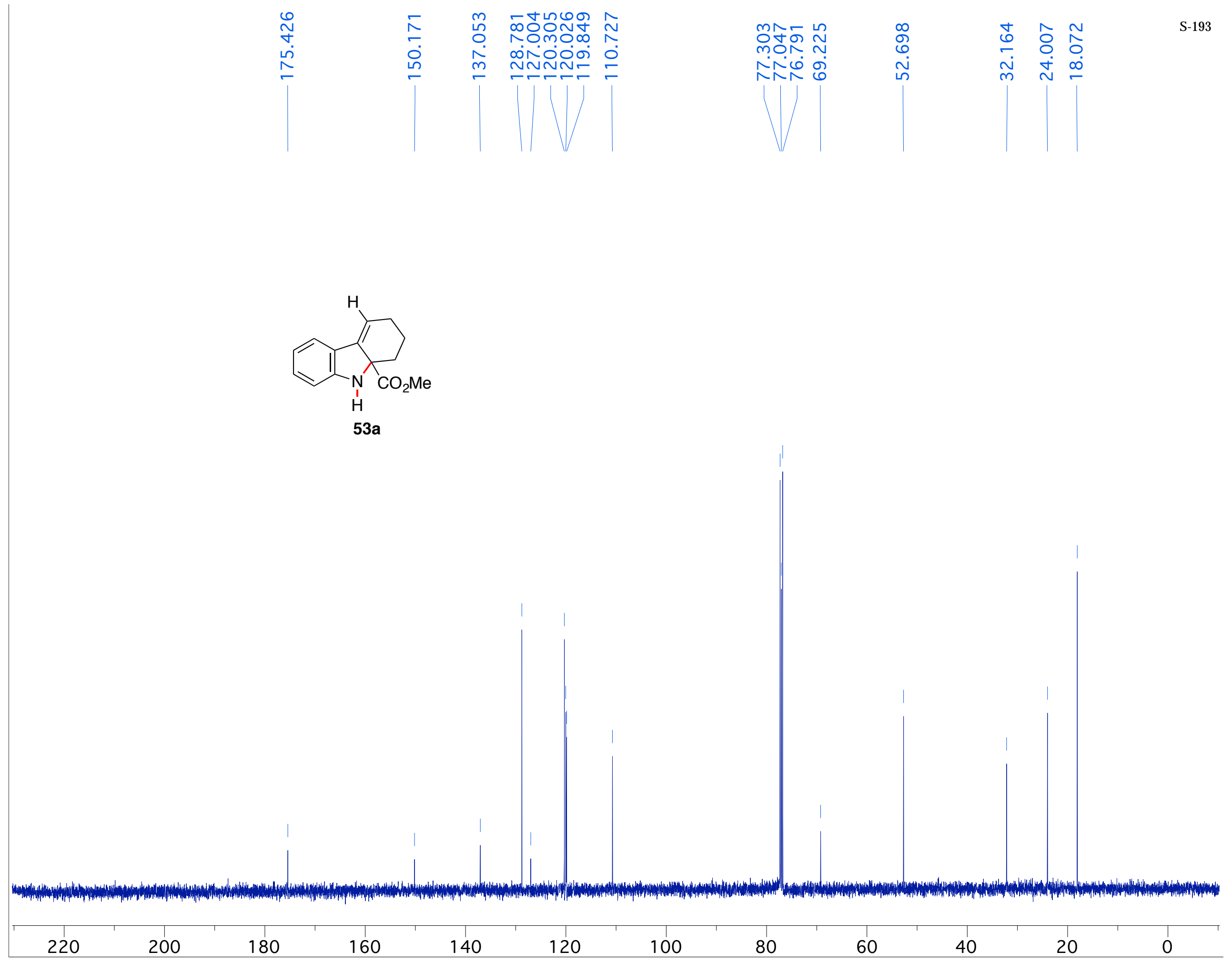



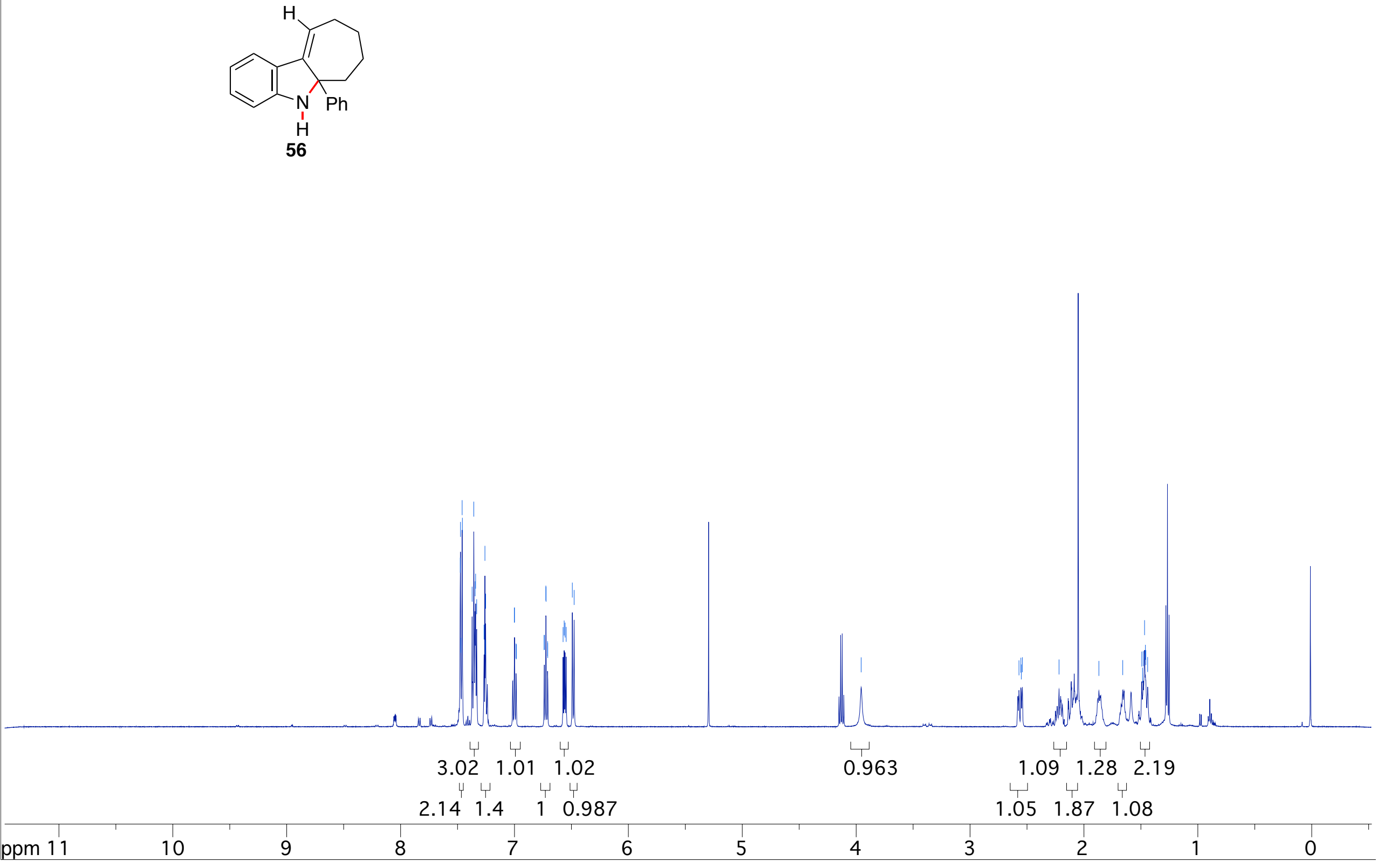

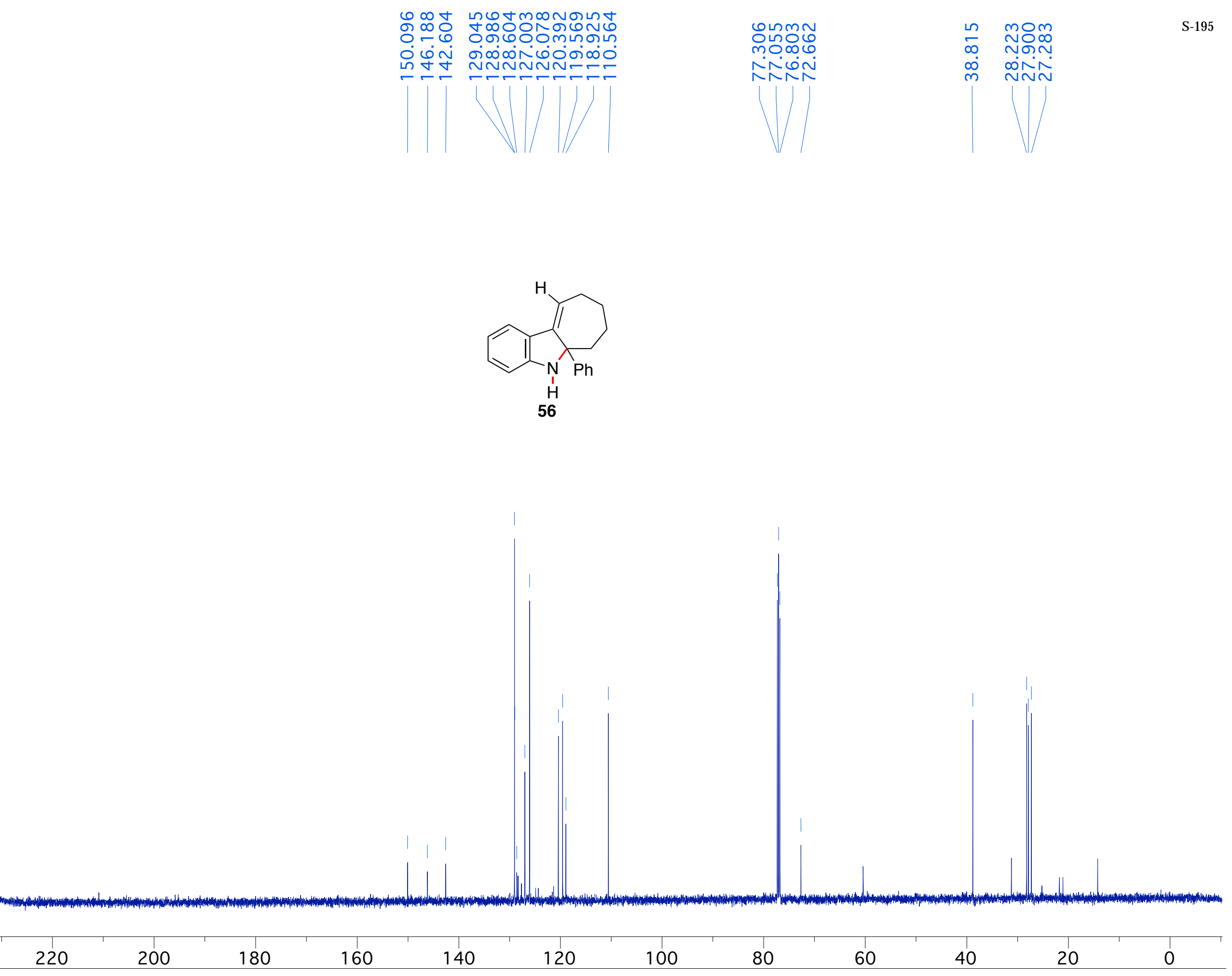